\title{
Einfluss von gelöstem Wasserstoff auf die Versetzungsbildung bei plastischer Verformung von Metallen
}

\author{
Dissertation \\ zur Erlangung des mathematisch-naturwissenschaftlichen Doktorgrades \\ „Doctor rerum naturalium“ \\ der Georg-August-Universität Göttingen
}

im Promotionsprogramm ProPhys

der Georg August University School of Science (GAUSS)

vorgelegt von

Martin Deutges

geboren in Duisburg

Göttingen 2016 


\section{Betreuungsausschuss:}

Prof. Dr. Reiner Kirchheim

Institut für Materialphysik

Georg-August-Universität Göttingen

Prof. Dr. Astrid Pundt

Institut für Materialphysik

Georg-August-Universität Göttingen

\section{Mitglieder der Prüfungskommission:}

Referent:

Prof. Dr. Reiner Kirchheim

Institut für Materialphysik

Georg-August-Universität Göttingen

Korreferent:

Prof. Dr. Michael Seibt

IV. Physikalisches Institut - Halbleiterphysik

Georg-August-Universität Göttingen

weitere Mitglieder der Prüfungskommission:

Prof. Dr. Astrid Pundt

Institut für Materialphysik

Georg-August-Universität Göttingen

Prof. Dr. Cynthia A. Volkert

Institut für Materialphysik

Georg-August-Universität Göttingen

PD Dr.-Ing. Helmut Klein

Abteilung Kristallographie im Geowissenschaftlichen Zentrum

Georg-August-Universität Göttingen

Prof. Dr. Hans Hofsäss

II. Physikalisches Institut - Kern- und Atomphysik

Georg-August-Universität Göttingen

Tag der mündlichen Prüfung: 20.01.2016 


\section{Inhaltsverzeichnis}

1 Einführung 1

2 Theoretische Grundlagen $\quad 5$

2.1 Das reale Metall: Entstehung, Bewegung und Interaktion von Defekten . . . . . . . 5

2.2 Lösung von Wasserstoff in Metallen . . . . . . . . . . . . . . . . . . . . . . . . 13

2.2.1 Übergang von Wasserstoff aus der Gasphase ins Metall . . . . . . . . . . . . 15

2.2.2 Übergang von Wasserstoff in Metall bei elektrolytischer Beladung . . . . . . 16

2.2.3 Diffusion von Wasserstoff in Metallen . . . . . . . . . . . . . . 17

2.3 Thermodynamik von Defekten und gelösten Atomen

Das Defactant-Konzept . . . . . . . . . . . . . . . . . . . . . . 19

2.4 Verwendete Metall-Wasserstoffsysteme . . . . . . . . . . . . . . . . . . 22

3 Experimentelle Methoden $\quad 25$

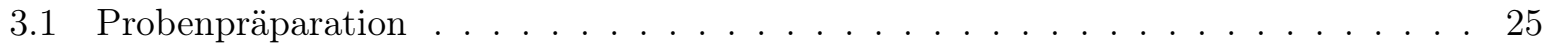

3.1.1 Elektrolytisches Beladen . . . . . . . . . . . . . . . . . . 25

3.1.2 Präparation von Metalloberflächen zur Untersuchung durch Nanoindentation 26

3.1.3 Präparation von Vanadiumsäulen im Mikrometerbereich . . . . . . . . . . 28

3.1.4 Präparation von TEM-Proben aus Palladiumblech . . . . . . . . . . . 28

3.2 Verformungsmethoden . . . . . . . . . . . . . . . . . . . . . . . . 29

3.2 .1 Nanoindentation . . . . . . . . . . . . . . . . . . . . . . . . 29

3.2 .2 Kaltwalzen von Palladiumblechen . . . . . . . . . . . . . . . . . . . 30

3.2 .3 Hochdruck-Torsion . . . . . . . . . . . . . . . . . . . . . . . . . 31

3.3 Probencharakterisierung . . . . . . . . . . . . . . . . . . . 32

3.4 Molekulardynamiksimulationen . . . . . . . . . . . . . . . . . . . 34 
4.1 Untersuchung des Einflusses von Wasserstoff auf die Versetzungsnukleation bei Nanoindentationsversuchen . . . . . . . . . . . . . . . . . . 35

4.2 Untersuchung der Versetzungsentwicklung in Molekulardynamiksimulationen von Nanoindentationsexperimenten . . . . . . . . . . . . . . . . . . . . 37

4.3 Untersuchungen von Defekten in Wasserstoffatmosphäre im ETEM . . . . . . . . . 39

4.4 Verformungsexperimente an Vanadium-Mikrosäulen . . . . . . . . . . . . . . . . . 40

4.5 Untersuchungen zur Bildung von Versetzungsnetzwerken am Beispiel von PalladiumWasserstoff Legierungen . . . . . . . . . . . . . . . . . . . . . . . 40

4.6 Bestimmung des Einflusses von Wasserstoff auf Palladium bei extremer Verformung 41

5 Ergebnisse

5.1 Einfluss von Wasserstoff auf einzelne Defekte . . . . . . . . . . . . . . . 43

5.1.1 Einfluss von Wasserstoff auf den Pop-In Effekt bei Nanoindentierung . . . . 43

5.1.2 Versetzungsentwicklung in MD-Simulationen von Nanoindentation . . . . . 46

5.1.3 Versetzungsanordnung unter Einfluss von Wasserstoff im ETEM . . . . . . 53

5.2 Einfluss von Wasserstoff auf die Versetzungsanordnung . . . . . . . . . . . . 58

5.2.1 Änderung der Verformungscharakteristik von Vanadium-Mikrosäulen . . . . 58

5.2.2 Entwicklung der Versetzungsdichte von kaltgewalzten Palladium-Wasserstofflegierungen . . . . . . . . . . . . . . . . . . 61

5.2.3 Untersuchungen zur Defektbildung in Palladium-Wasserstofflegierungen bei extremer Verformung . . . . . . . . . . . . . . . . . . . . 62

6 Diskussion

6.1 Einfluss von Wasserstoff auf einzelne Versetzungen _ . . . . . . . . . . . . 65

6.2 Einfluss von Wasserstoff auf Versetzungsnetzwerke . . . . . . . . . . . . . 71

6.3 Einfluss von Wasserstoff auf die Linienenergie . . . . . . . . . . . . . . . 75

6.4 Zusammenfassung der beobachteten Einflüsse von Wasserstoff auf die Defektbildung . . . . . . . . . . . . . . . . . . 78

6.5 Übergang zu makroskopischen Vorgängen ausgelöst durch gelösten Wasserstoff . . . . . . . . . . . . . . . . . . . 79 
9.1 Möglichkeiten zur Protokollierung der Versetzungsentwicklung während Zugversuchen 85

9.2 Weitere Messergebnisse . . . . . . . . . . . . . . . . . . . . . 88

9.2.1 Untersuchungen zur Defektbildung in Palladium-Wasserstofflegierungen durch Walzen . . . . . . . . . . . . . . . . . . . 88

9.2.2 Untersuchungen zur Defektbildung in Palladium-Wasserstofflegierungen bei extremer Verformung . . . . . . . . . . . . . . . . 89

9.3 Analyse der Abgleitung von Mikrosäulen unter Verwendung des Schmidfaktors . . . . . . . . . . . . . . . . . . . . . 97

9.4 Übersicht über betreute Bachelorarbeiten . . . . . . . . . . . . . . . . . . . 98

9.4.1 Annegret Lehmberg - Untersuchung der Volumenänderung von Eisen nach Wasserstoffbeladung . . . . . . . . . . . . . . . . 9 98

9.4.2 Malte Lenz - Zusammenhang zwischen der Mikrostruktur und den mechanischen Eigenschaften sprengverschweißter Stähle . . . . . . . . 101

9.4.3 Moritz Roscher - Einfluss der Wasserstoffbeladung auf die mechanischen Eigenschaften von Metallen . . . . . . . . . . . . . . . . . . . . 104

9.5 Veröffentlichungen . . . . . . . . . . . . . . . . . . . . 113 


\section{$1 \quad$ Einführung}

Wasserstoff als kleinstes aller Elemente besitzt die Fähigkeit sich in vielen Metallen zu lösen, was in vielen Fällen eine Veränderung der mechanischen Eigenschaften verursacht. Dies kann dazu führen, dass metallische Bauteile versagen. Dieser Effekt wird Wasserstoffversprödung genannt [1] und stellt ein erhebliches wirtschaftliches Problem dar.

Wasserstoffversprödung tritt oft dann auf, wenn wasserstoffhaltige Flüssigkeiten oder Gase z. B. (Salz-)Wasser [2, 3], Schmieröle [4, 5], Erdöl und Erdgas [6] in Kontakt mit ungeschützten Metalloberflächen kommen. Ein bedeutendes aktuelles Beispiel hierfür sind Offshore-Anlagen, wie z.B. Ölplattformen oder Windkraftanlagen. Im Betrieb ist die Anlage aggressivem Meerwasser ausgesetzt, wodurch Korrosion begünstigt wird. Ein weiteres Problem, welches bei der Konstruktion dieser Anlagen auftritt, ist die Lösung von Wasserstoff in Schweißnähten, der diese, begünstigt durch die zyklische Belastung des Wellengangs, schwächt [7]. Neben der Lösung von Wasserstoff durch korrosive Prozesse wird die Wasserstoffaufnahme bspw. auch durch mikrobiologische Vorgänge begünstigt [8]. Die Lösung von Wasserstoff führt somit zur frühzeitigen Alterung von strukturellen Bauelementen. Ein anderes Problem, was sich im speziellen bei Offshore-Windkraftanlagen ergibt, ist die Versprödung von bewegten Teilen wie z. B. Wälzlager. Reiben Metallteile aufeinander, gibt es verschiedene Mechanismen, die die Wasserstoffbildung begünstigen. Wärme, die durch Reibung entsteht, kann Meerwasser oder auch Schmiermittel zersetzen und Wasserstoff freisetzen. Werden Oxid- oder Schutzschichten beschädigt, liegt reines Metall vor, welches in vielen Fällen sehr reaktiv ist. Dies kann mit Schmiermitteln reagieren, wodurch Wasserstoff freigesetzt wird [9]. Neben der Reibung, bzw. der Reaktion von Metallen mit Flüssigkeiten kann Wasserstoff auch dadurch entstehen, dass statische elektrische Ladungen vorliegen, welche bspw. durch Reibung oder durch Kontakt unterschiedlicher Metalle entstehen können. Dies führt zur elektrolytischen Erzeugung von Wasserstoffatomen [4, 5].

Der gelöste Wasserstoff trägt somit über zwei Prozesse zur Alterung von Offshore-Anlagen bei: Zum einen können Schäden an strukturellen Bauelementen entstehen, zum anderen wird die Lebensdauer von bewegten Teilen wie z. B. Wälzlager herabgesetzt, deren Austausch aufwendig und teuer ist.

Ein weiteres Beispiel für die Problematik der Wasserstoffversprödung ist die Förderung von Öl und Gas. Da die vorhandenen Lagerstätten zur Neige gehen, müssen auch weniger attraktive Vorkommen erschlossen werden. Ein Grund für die niedrige Attraktivität solcher Vorkommen ist der höhere Anteil von Schwefelwasserstoff $\left(\mathrm{H}_{2} \mathrm{~S}\right)$ im Rohstoff. Solche Rohstofflager werden als ,sauer" bezeichnet [6]. Im Kontakt mit Metallen spalten sich Wasserstoffatome ab, wobei der dabei entstehende Schwefel zusätzlich als Katalysator zur Aufnahme von Wasserstoff ins Metall wirkt [6]. Die gelösten Wasserstoffatome führen zur Versprödung des Metalls, was letztendlich, gepaart mit den hohen Belastungen beim Bohren, zur Zerstörung des Bohrers führt. Dieses Problem betrifft nicht nur den Bohrer und das Bohrgestänge, sondern auch alle anschließenden Anlagen zur Aufbereitung. Daher wird ein enormer Aufwand betrieben, um $\mathrm{H}_{2} \mathrm{~S}$ aus dem Rohstoff zu entfernen [10, 11].

Diese Beispiele zeigen, dass viele verschiedene Effekte durch Wasserstoff verursacht werden, die unter dem Begriff Wasserstoffversprödung zusammengefasst werden [1]. Daher ist das Verhalten von Metallen unter Wasserstoffeinfluss seit vielen Jahren ein wichtiges Forschungsfeld [1, 12, 13]. Makroskopisch gesehen begünstigt Wasserstoff die Bildung von Rissen [14] oder führt zur geringerer Festigkeit, was zum Bruch führen kann [15, 16]. Weitere Erscheinungsformen sind die Bildung von Hohlräumen im Material und Wölbungen an der Oberfläche $[17,18]$.

Obwohl die Versprödung durch Wasserstoff seit langem untersucht wird, gibt es noch keine einheitliche Beschreibung aller beobachteten Phänomene. Für die Beschreibung mikroskopischer Vorgänge werden verschiedene Mechanismen diskutiert [19, 20]. 
Ein fortgeschrittenes Modell, welches die Auswirkungen von Wasserstoff auf Versetzungen beschreibt, ist das sog. HELP Modell (engl.: Hydrogen-Enhanced Localised Plasticity), welches von Birnbaum vorgestellt wurde [21-23]. Dieses Modell wurde aufgrund der Beobachtung in einem Transmissionselektronenmikroskop (TEM) entwickelt [23-26]. Es wurde gezeigt, dass Versetzungen in dünnen unter Spannung stehenden Metallfilmen sich zu bewegen beginnen, sobald eine Wasserstoffatmosphäre aufgebaut wird. Dabei wurde auch beobachtet, dass Versetzungen ihren Abstand zueinander verringern. Diese erhöhte Aktivität von Versetzungen wird gelöstem Wasserstoff zugeschrieben. Dieser bewirkt, im Rahmen dieses Modells, dass die Spannungsfelder von Versetzungen und anderen Kristallbaufehlern abgeschirmt werden. Versetzungen können sich so ungehinderter bewegen und somit besser auf äußere mechanische Spannungsfelder reagieren.

Der Grund, warum gelöster Wasserstoff in Metall mit Versetzungen (und anderen Kristallbaufehlern) interagiert, ist die daraus resultierende Reduzierung der Defektenergie durch die Segregation von Wasserstoff an Defekten [27, 28]. Dadurch wird bspw. die Bildung von Kinken innerhalb von Versetzungslinien, welche für die Bewegung von Versetzungen durch das Metall nötig sind, erleichtert. Die dieser Interaktion zugrunde liegenden thermodynamischen Vorgänge, die zur Reduzierung der Defektenergie führen, werden durch das von Kirchheim vorgestellte Defactant-Konzept (engl.: defect acting agent) beschrieben [27-30]. Anschaulich erklärt sich die Interaktion von Wasserstoff mit Defekten mit seiner Größe. Obwohl Wasserstoff im Vergleich zu anderen Atomen klein ist, dehnt der gelöste Wasserstoff das umliegende Gitter auf. Um Defekte herum kann das Gitter auch durch die vom Defekt ausgehenden Spannungen verzerrt sein, wodurch die für Wasserstoff möglichen Plätze vergrößert sind. Wenn Wasserstoff zu diesen geweiteten Plätzen diffundiert, wird die Gesamtdehnung des Gitters reduziert. Dadurch kann die Gesamtenergie von Defekt und Wasserstoff reduziert werden. Neben diesen mechanischen Beiträgen zur Gesamtenergie beeinflusst das Wasserstoffatom weitere Faktoren, wie z.B. die lokale Elektronendichte. Das Defactant-Konzept ordnet diese Energiereduzierung der Defektenergie zu und kann somit die erhöhte Aktivität von Versetzungen unter Wasserstoffeinfluss erklären.

Das Ziel dieser Arbeit ist es, die Vorgänge, die in Metall durch Wasserstoff ausgelöst werden, auf der Ebene der Versetzungen zu beschreiben. Um einen breiten Überblick über die Vorgänge zu erlangen, wurde der Effekt von Wasserstoff auf die Bildung und die Bewegung von Versetzungen, bis hin zur Bildung von Versetzungsnetzwerken bei verschiedenen Metallen untersucht.

Für die Untersuchungen wurden hauptsächlich Palladium und Vanadium als Modellmaterialien verwendet. Diese Metalle unterscheiden sich hinsichtlich ihrer Gitterstruktur und erlauben es so einen breiten Überblick über prinzipielle Vorgänge im kubisch flächenzentrierten (kfz) Gitter (Palladium) bzw. kubisch raumzentrierten (krz) Gitter (Vanadium) zu erhalten. Beide Metalle erlauben es zudem große Mengen an Wasserstoff in ihnen zu lösen. Des Weiteren ist es möglich die Interaktion von Wasserstoff mit Versetzungen zu untersuchen, ohne dass andere Effekte, wie z.B. Oxidation, überlagern.

Für die Untersuchung des Einflusses von Wasserstoff auf einzelne Versetzungen wurden zwei Methoden verwendet. Einerseits wurde der direkte Einfluss von Wasserstoff auf die Bildungsenergie von Versetzungen durch die lokale Verformung von einkristallinen Materialien mit Hilfe von Nanoindentation untersucht. Andererseits wurde der Einfluss von Wasserstoff auf die Bewegung von einzelnen Versetzungen mit Hilfe eines Environmental Transmissionselektronenmikroskops (ETEM) beobachtet.

Um den Einfluss von Wasserstoff auf die Interaktion von wenigen Versetzungen miteinander zu untersuchen wurden Mikrosäulen verformt. Die Verformung von Proben in dieser Größenordnung stellt den Übergang von der Verformung auf atomarer Ebene durch einzelne Versetzungen zu makroskopischen Verformungen dar.

Zur Übertragung der gewonnenen Erkenntnisse auf industrielle Prozesse wurde eine typische Verformungsmethode, das Kaltwalzen, verwendet, um den Einfluss von Wasserstoff auf die Defektbildung und Anordnung bei massiver Verformung zu zeigen. 
Daher gliedert sich die Arbeit in folgende Teile: Zunächst wird im Abschnitt 2 auf grundlegende theoretische Hintergründe eingegangen. Hierbei werden in Kapitel 2.1 zunächst verschiedene Aspekte der Versetzungsdynamik, sowie der Interaktion von Fremdatomen mit Versetzungen beschrieben. Danach wird in Kapitel 2.2 auf die verschiedenen Möglichkeiten des Lösungsvorgangs von Wasserstoff in Metallen eingegangen. Daran anschließend werden die thermodynamischen Zusammenhänge der Defekt - Fremdatom Interaktion, das Defactant-Konzept, dargestellt. Als letzter Teil des theoretischen Hintergrunds werden die verwendeten Metall-Wasserstoffsysteme kurz beschrieben (Kapitel 2.4).

Auf den theoretischen Abschnitt folgt zunächst ein Überblick über die verwendeten experimentellen Methoden in Abschnitt 3 und danach die Beschreibung der durchgeführten Versuche in Abschnitt 4.

In Abschnitt 5 werden die Ergebnisse dieser Versuche vorgestellt. Dabei werden die Ergebnisse verschiedener Untersuchungen zum Einfluss von Wasserstoff auf einzelne Versetzungen in Kapitel 5.1 und die Ergebnisse zu Untersuchungen des Einflusses von Wasserstoff auf die Interaktion von Versetzungen miteinander und auf Versetzungsnetzwerke in Kapitel 5.2 vorgestellt.

Die Diskussion der Ergebnisse folgt in Abschnitt 6. Zunächst werden die einzelnen Ergebnisse in Kapitel 6.1 bzw. in Kapitel 6.2 im Rahmen des Defactant-Konzepts analysiert. Daran anschließend wird der Einfluss von Wasserstoff auf die Linienenergie von Versetzungen bei den einzelnen Versuchen in Kapitel 6.3 herausgearbeitet. Die bis hierhin erhaltenen Erkenntnisse werden in Kapitel 6.4 zusammengefasst. Abschließend wird in Kapitel 6.5 aufgezeigt wie die gewonnenen Erkenntnisse der Wasserstoff - Versetzungsinteraktion in Zusammenhang mit Beobachtungen auf makroskopischer Ebene gebracht werden können. 


\section{Theoretische Grundlagen}

Im vorliegenden Kapitel wird die Defektentstehung in Metallen bei Anwesenheit von Fremdatomen dargestellt. Dabei wird die Entstehung von Versetzungen unter Einfluss von Wasserstoff besonders hervorgehoben.

Zunächst wird der atomare Aufbau und die Entstehung von Defekten in Metall behandelt. Anschließend wird der Mechanismus der Lösung und Diffusion von Wasserstoff in Metall dargestellt. Abschließend wird auf das Defactant-Konzept (engl.: defect acting agent) eingegangen, welches die Interaktion zwischen gelösten Atomen mit Defekten im Metall beschreibt und somit die Grundlage dieser Arbeit darstellt.

\subsection{Das reale Metall: Entstehung, Bewegung und Interaktion von Defekten}

In einem kristallinen Material wird jede Abweichung vom idealen Aufbau der Kristallstruktur als Defekt bezeichnet. Defekte haben einen wesentlichen Einfluss auf die mechanischen Eigenschaften makroskopischer Objekte. Durch gezieltes Einbringen von Defekten z. B. durch Verformung oder durch Hinzufügen von Fremdatomen können mechanische Eigenschaften verbessert werden. Wird beispielsweise Kohlenstoff zu Eisen legiert, entsteht Stahl. Durch weitere Verformung kann das Material weiter optimiert werden. So zeigt sich bei kalt gezogenen perlitischen Stahldrähten [31-33] eine erhöhte Zugfestigkeit und Bruchzähigkeit.

Um die ideale Defektdichte hinsichtlich der mechanischen Anforderungen zu erreichen, können durch gezieltes Erwärmen (Auslagern) Defekte abgebaut oder umstrukturiert werden [34-36]. So kann durch Auslagern die Diffusion von Kohlenstoff in verformten Eisen-Kohlenstofflegierungen erhöht werden. Dies führt dazu, dass sich der Kohlenstoff an Korngrenzen anlagert [33, 37].

Im Gegensatz zu Kohlenstoff führt gelöster Wasserstoff bei vielen Metallen zu Versprödungserscheinungen. Dieser Effekt wird daher als Wasserstoffversprödung bezeichnet [1, 12, 30]. Wasserstoff hat die Fähigkeit, bedingt durch seine geringe Größe im Vergleich zu anderen Atomen, sich auf Zwischengitterplätzen zwischen den Atomen des Metalls zu bewegen [38, 39]. Bereits bei Raumtemperatur diffundiert Wasserstoff im Metall Distanzen von mehreren $100 \mu$ m innerhalb weniger Minuten [40]. Wird genügend Wasserstoff angeboten, reichen allein diese Fähigkeiten aus, um Risse in einigen Metallen ohne äußere Kräfte hervorzurufen, z. B. in Eisen [17, 18] oder Aluminium [41, 42]. Wirken zusätzlich mechanische Spannungen auf ein Metall, haben schon kleinste Mengen an Wasserstoff großen Einfluss auf die Defektbildung [14, 40, 43, 44].

In diesem Kapitel wird die Entstehung verschiedener Defekttypen und deren Interaktion mit gelösten Fremdatomen beschrieben. Erklärt wird das beschriebene Verhalten thermodynamisch durch das Defactant-Konzept, welches in Kapitel 2.3 beschrieben wird.

Außerdem wird auf das Verhalten der Defekte bei angelegten mechanischen Spannungen eingegangen.

\section{Punktdefekte}

Bei reinen Metallen gibt es zwei Arten von Punktdefekten, zum einen kann ein Atom fehlen, was zu einer Leerstelle führt, zum anderen kann sich ein Metallatom auf einem Zwischengitterplatz befinden. Sind Fremdatome im Metall vorhanden gibt es mehrere weitere Möglichkeiten. Die Fremdatome können reguläre Gitterplätze einnehmen oder sich auf Zwischengitterplätzen befinden. Des Weiteren können spezielle Kombinationen bzw. Anordnungen mehrerer Punktdefekte entstehen. Den meisten Defekttypen ist gemein, dass das umgebende Metallgitter verzerrt wird. Aus thermodynamischen Gründen befinden sich in einem Material immer Punktdefekte [45]. Dies liegt daran, dass die Entropie $S$ durch Defekte erhöht wird, was zu einer Reduzierung der Freien Enthalpie $G$ führt. Für die Gleichgewichtskonzentration $c_{P}$ an Punktdefekten ergibt sich [45]:

$$
c_{P}=\exp \left(-\frac{G_{B}}{k T}\right)
$$


Dabei ist $G_{B}$ die Bildungsenthalpie des Defektes, $k$ die Boltzmann-Konstante und $T$ die Temperatur. Beispielsweise liegt die Leerstellenkonzentration in Metallen am Schmelzpunkt bei typischerweise $c_{P}=10^{-4}-10^{-3}$ Leerstellen pro Metallatom [46], was einem Abstand der Leerstellen (im kfz-Gitter) von 6-14 Einheitzellen des Kristallgitters entspricht.

Durch das Hinzufügen von Fremdatomen können Leerstellen stabilisiert werden (siehe Kapitel 2.3). So wurde von Fukai et al. [47] gezeigt, dass sich sehr hohe Leerstellenkonzentrationen (bis zu 0,1 Leerstellen pro Metallatom) erzeugen lassen, wenn Palladium hohen Wasserstoffgasdrücken und Temperaturen (5 GPa und $1168 \mathrm{~K}$ ) ausgesetzt wird. Durch Verformen von Palladium-Wasserstofflegierungen mittels HPT (engl.: High Pressure Torsion) (siehe Kapitel 3.2.3) können ähnlich hohe Leerstellenkonzentrationen auch bei Raumtemperatur erzeugt werden [48].

Ein weiteres Beispiel für die Erhöhung der Leerstellenkonzentration durch gelöste Fremdatome ist kalt gezogener Stahl [49]. Wird perlitischer Stahl gezogen, bilden sich Leerstellen-Kohlenstoff Agglomerate in einer Konzentration von der Größenordnung $10^{-4}$.

\section{Versetzungen ${ }^{1}$}

Eine Versetzung in einem kristallinen Material ist eine lokal begrenzte Abweichung der Stapelung einzelner atomarer Ebenen vom Idealzustand. Durch die Bildung einer Versetzung kann das Material mechanische Spannungen abbauen und so plastisch verformt werden. Eine Versetzung kann im einfachsten Fall eine eingeschobene Halbebene sein (vgl. Abbildung 1). Diese Art von Versetzung wird Stufenversetzung genannt. Der eigentliche Defekt ist hierbei nur das Ende der eingeschobenen Halbebene, da die übrige Gitterstruktur erhalten bleibt. In der Umgebung eines solchen Defekts liegen nur elastische Verzerrungen vor.

Um eine Versetzung zu charakterisieren, wird der sog. Burgersvektor verwendet. Dieser kann auf atomarer Ebene bestimmt werden, indem (entfernt von der Versetzungslinie) um die Versetzungslinie in einem Rechteck die Atome gezählt werden. Dabei ergibt sich, dass ein Paar der sich gegenüberliegenden Seiten des Rechteckes nicht gleich lang sind. Der Unterschied in Länge und Richtung zwischen den zwei Seiten wird als Burgersvektor definiert. Bei einer Stufenversetzung steht dieser senkrecht auf der Versetzungslinie und liegt somit auf der Gleitebene der Versetzung.
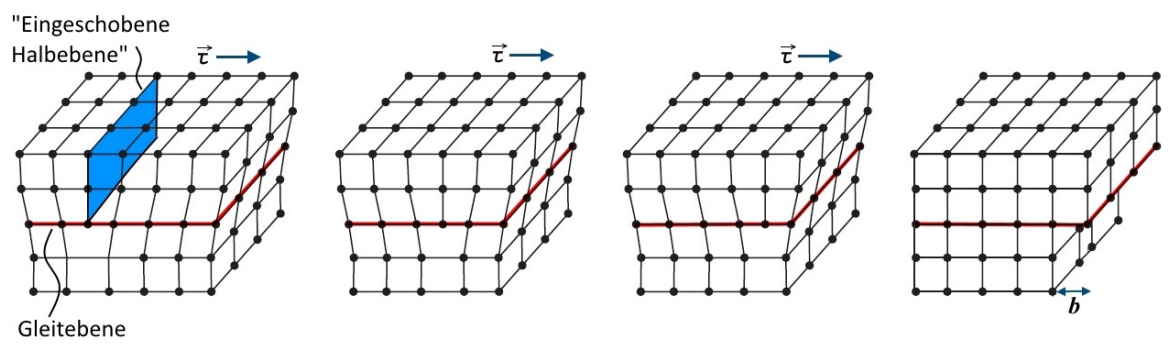

Abb. 1 Wird eine einkristalline Probe einer Scherkraft ausgesetzt, bildet sich im einfachsten Fall eine Stufenversetzung. Diese bewegt sich nach und nach durch das Material mit Hilfe einer eingeschobenen Halbebene [50]. Die Ebene senkrecht zur Halbebene, entlang der sich die Versetzung bewegt, wird Gleitebene genannt.

Ein weiterer wichtiger Typ von Versetzung ist die sog. Schraubenversetzung. Zur Veranschaulichung (vgl. Abbildung 2) dieses Versetzungstyps wird ein idealer Kristall entlang einer kristallinen Ebene zur Hälfte aufgeschnitten, wobei das Ende des Schnittes die Versetzungslinie ist. Nun werden die aufgeschnittenen Teile um einen Ebenenabstand gegeneinander verschoben und wieder zusammengefügt. Wird hier ein Burgersvektorumlauf durchgeführt, kommt man nicht zum Ausgangspunkt zurück, sondern bewegt sich auf einer Schraubenbahn. Der Burgersvektor ist demnach parallel zur Versetzungslinie [45].

\footnotetext{
${ }^{1}$ Im folgenden Abschnitt wird vielfach auf kristalline Richtungen und Ebenen eingegangen. Hierzu werden die üblichen Konventionen zur Beschreibung der millerschen Indizes verwendet: $(h k l)$ Spezifische Ebene; $\{h k l\}$ symmetrisch äquivalente Ebenen; [hkl] spezifische Richtung; $\langle h k l\rangle$ symmetrisch äquivalente Richtungen [45]
} 

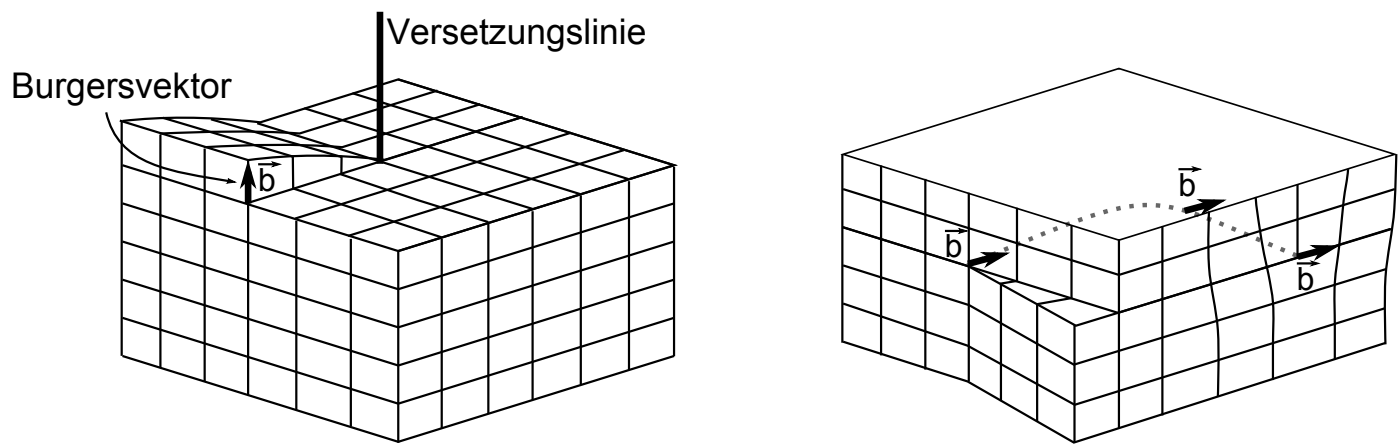

Abb. 2 Links: Schematische Darstellung einer Schraubenversetzung; Rechts: Ausschnitt einer Versetzungslinie, welche den Versetzungscharakter ändert. Darstellung nach [45].

Ein weiterer Aspekt einer Versetzung ist, dass es sich um einen sog. topologischen Defekt handelt. Das heißt, dass eine Versetzungslinie nicht im ungestörten Kristall beginnen bzw. enden kann. Eine Versetzung beginnt entweder an einer Korn- bzw. Phasengrenze ${ }^{2}$ oder einer Oberfläche oder es bilden sich Versetzungsringe aus. Für die Bildung eines Versetzungsrings wird eine Kombination von Stufen- und Schraubenversetzungen gebraucht (vgl. Abbildung 2). Dies zeigt, dass eine Versetzung ihren Charakter entlang ihrer Versetzungslinie verändern kann. Diese Eigenschaft befähigt Versetzungen dazu einem Spannungsfeld zu folgen und um Hindernisse, wie z. B. andere Versetzungen, herum geführt zu werden.

In Abbildung 3 wird dargestellt, wie eine Versetzung sich durch das Ausbilden von sog. Kinken bzw. Jogs bewegen kann. Kinken und Jogs sind Stufen innerhalb einer Versetzungslinie. Es handelt sich also um Abweichungen bzw. Defekte der idealen Versetzungslinie. Eine Versetzung ist ein nicht thermodynamisch stabiler Defekt, wohingegen (Doppel-) Kinken aufgrund ihrer niedrigen Bildungsenergie sehr wohl stabil sind. Es stellt sich analog zu Leerstellen im Festkörper eine Gleichgewichtskonzentration von Kinken auf Versetzungen ein [51].

Zusammengefasst gibt es zwei Sorten von Kinken, thermisch aktivierte und Kinken die aufgrund von geometrischen Gegebenheiten vorhanden sind (siehe Abbildung 3a). Durch Kinken kann eine Versetzung innerhalb ihrer Gleitebene fortbewegt werden (Abbildung 3b), Jogs ändern die Gleitebene, siehe Abbildung 3c [51].

Für die spätere Beschreibung der Interaktion von Fremdatomen mit Versetzungen ist anzumerken, dass Kinken einer Schraubenversetzung vom Charakter her einer Stufenversetzung entsprechen.
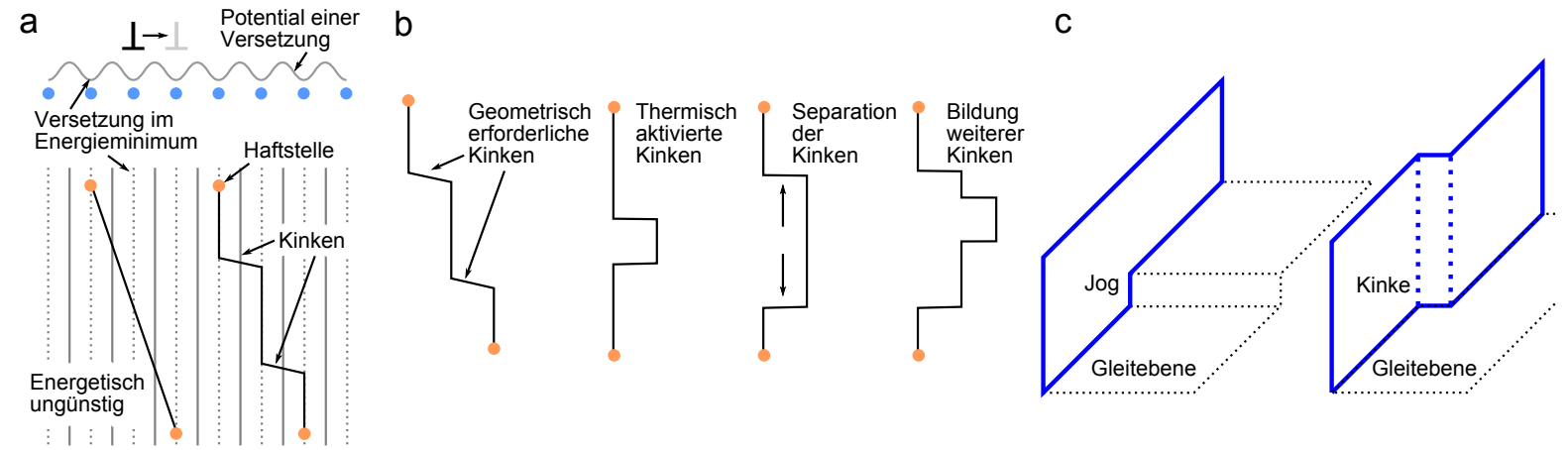

Abb. 3 Darstellung von Kinken und Jogs. Bild a zeigt das energetische Potential einer Stufenversetzung. Ist die Versetzung an zwei Haftstellen fixiert, müssen Kinken entstehen, damit die Versetzungslinie größtenteils dem Energieminimum folgen kann. Abbildung $\boldsymbol{b}$ zeigt den Unterschied zwischen geometrisch notwendigen und thermisch aktivierten (Doppel-) Kinken. Des Weiteren wird gezeigt, wie durch Bewegung der Kinken die gesamte Versetzung bewegt werden kann. Abbildung c zeigt den Unterschied zwischen Jogs und Kinken. Darstellung nach [51].

\footnotetext{
${ }^{2}$ Ein Korn ist ein einheitlich kristalliner Bereich im Material. Der Übergang von einem Korn zu einem anderen wird Korngrenze genannt. Weist das andere Korn jedoch eine andere Struktur oder chemische Zusammensetzung auf, handelt es sich um eine Phasengrenze.
} 


\section{Versetzungen im kfz Gitter}

In dieser Arbeit wurden kubisch flächenzentrierte (kfz) und kubisch raumzentrierte (krz) Metalle untersucht. Daher werden für diese Strukturen die typischen Ausbildungen von Versetzungen und deren Bewegungsmöglichkeiten erläutert.

Für die Linienenergie $E_{L}$ von Versetzungen pro Burgersvektor $b$ ergibt sich [51]:

$$
\begin{aligned}
E_{L, \text { Schraube }} & =\frac{G b^{2}}{4 \pi} \ln \left(\frac{R}{r_{0}}\right) \\
E_{L, \text { Stufe }} & =\frac{G b^{2}}{4 \pi(1-\nu)} \ln \left(\frac{R}{r_{0}}\right)
\end{aligned}
$$

Hierbei bezeichnet $G$ den Schermodul und $\nu$ die Poissonzahl. Die Parameter $R$ und $r_{0}$ resultieren aus der Reichweite des Spannungsfelds der Versetzung, $R$ ist die Reichweite ins Material und $r_{0}$ der Radius des Versetzungskerns. Diese Gleichungen zeigen, dass die Bildung von (vollständigen) Stufenversetzungen energetisch ungünstig ist ${ }^{3}$. Jedoch kann die Energie einer Versetzung herabgesetzt werden, indem sich die Versetzung in zwei partielle Versetzungen aufteilt. Da die Linienenergie proportional zu $b^{2}$ ist, muss für die Aufteilung gelten:

$$
b_{\text {vor }}^{2} \leq b_{\text {nach }, 1}^{2}+b_{\text {nach }, 2}^{2}
$$

Eine Versetzung wird „,vollständig“" genannt, wenn die Stapelung der einzelnen Ebenen im Material unverändert bleibt, nachdem die Versetzung hindurch bewegt wurde. Nachdem eine „partielle“ Versetzung durch das Material gewandert ist, bleibt ein Stapelfehler zurück (vgl. Abbildung 4).

Im kfz Gitter hat eine vollständige Stufenversetzung einen Burgersvektor von $b=\frac{a}{2}\langle 110\rangle$, mit $a$ dem Gitterparameter des Metalls. Durch die Bildung eines Stapelfehlers (vgl. Abbildung 4) kann die Energie der Stufenversetzung herabgesetzt werden. Hierbei wird der Beginn bzw. das Ende des Stapelfehlers durch partielle Versetzungen gebildet. Der Burgersvektor einer partiellen Versetzung ist von der Form $b=\frac{a}{6}\langle 112\rangle$, damit ergibt sich für die möglichen Burgersvektoren folgende Aufspaltungsreaktion:

$$
\frac{a}{2}\langle 110\rangle \rightarrow \frac{a}{6}\langle 211\rangle+\frac{a}{6}\langle 12 \overline{1}\rangle
$$
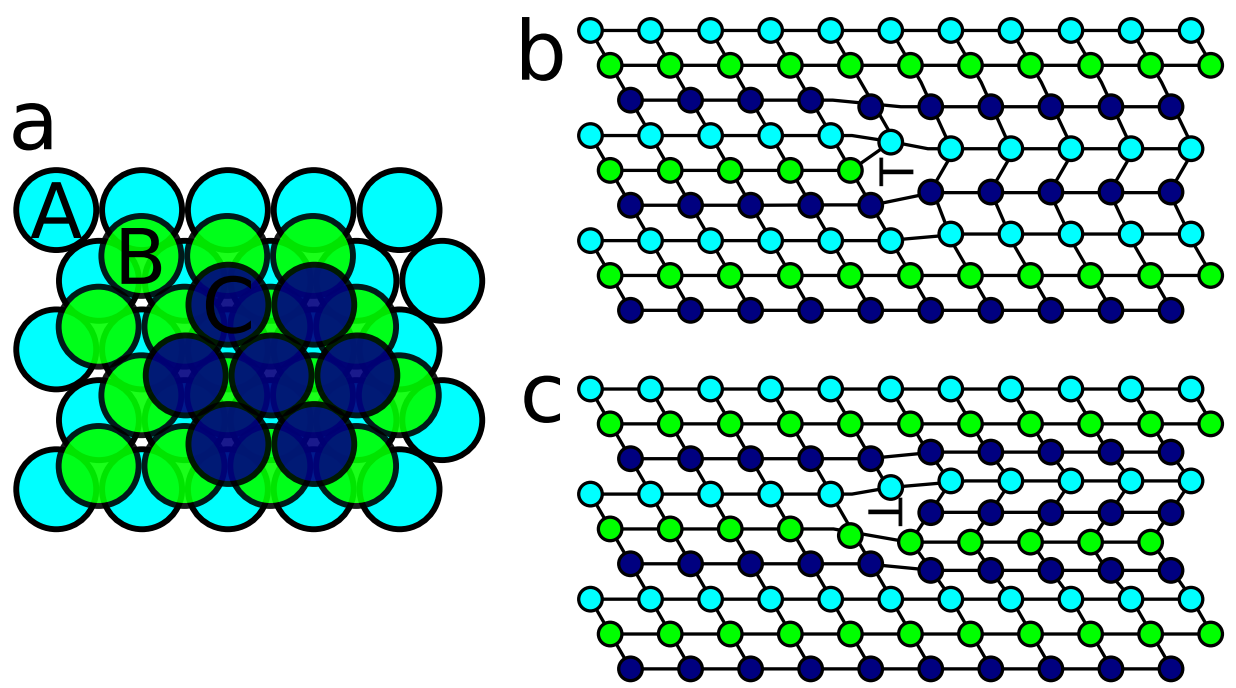

Abb. 4 Darstellung der Atome in einem kfz-Kristall [51]. Abbildung a zeigt die Stapelreihenfolge einzelner atomarer $\{111\}$-Ebenen. Abbildung $\boldsymbol{b}$ und $\boldsymbol{c}$ zeigen die atomaren Ebenen aus einer $<110>$-Richtung. Abbildung $\boldsymbol{b}$ zeigt einen intrinsischen Stapelfehler (entfernte Halbebene) und $\boldsymbol{c}$ einen extrinsischen (eingefügte Halbebene) Stapelfehler.

\footnotetext{
${ }^{3}$ Typische Werte der Poissonzahl $\nu$ liegen bei Metallen zwischen 0,3 und 0,4 .
} 
Dem Energiegewinn, der sich durch die Aufspaltung in zwei partielle Versetzungen ergibt, wirkt die Stapelfehlerenergie $\gamma$ entgegen. Daher können die partiellen Versetzungen nicht beliebig weit auseinander liegen. Es ergibt sich ein Gleichgewichtsabstand $d$ [51]:

$$
d=\frac{G b^{2}}{4 \pi \gamma}
$$

Für Palladium ergibt sich der Abstand und damit der Durchmesser eines von einer Stufenversetzung erzeugten Stapelfehlers $\mathrm{zu}^{4} d=1,47 \mathrm{~nm}$. Dieses Verhalten kann durch Molekulardynamiksimulationen demonstriert werden (siehe Kapitel 3.4). Wird auf eine Grenzfläche, dies können Oberflächen, Korngrenzen oder Phasengrenzen sein, eine (ungleichmäßige) Kraft ausgeübt, werden Versetzungsringe gebildet, die sich ins Material ausbreiten und an der Grenzfläche eine Stufe zurücklassen. Abbildung 5 zeigt eine im Rahmen dieser Arbeit simulierte Palladiumoberfläche, welche mit einem Nanoindenter verformt wurde (siehe Kapitel 5.1.2). Zur Darstellung wurden alle Atome entfernt, welche sich in einer perfekten kfz Umgebung befinden. Es bleiben Oberflächenatome und Stapelfehler, welche die einzelnen Versetzungen bilden, zurück. Es ist zu erkennen, dass sich die einzelnen Stapelfehler so anordnen, dass Versetzungsringe beginnend an der Oberfläche entstehen. Dabei beträgt der Durchmesser der Stapelfehler in [110]-Richtung 3-6 Atomlagen, was $0.82-1.65 \mathrm{~nm}$ entspricht.

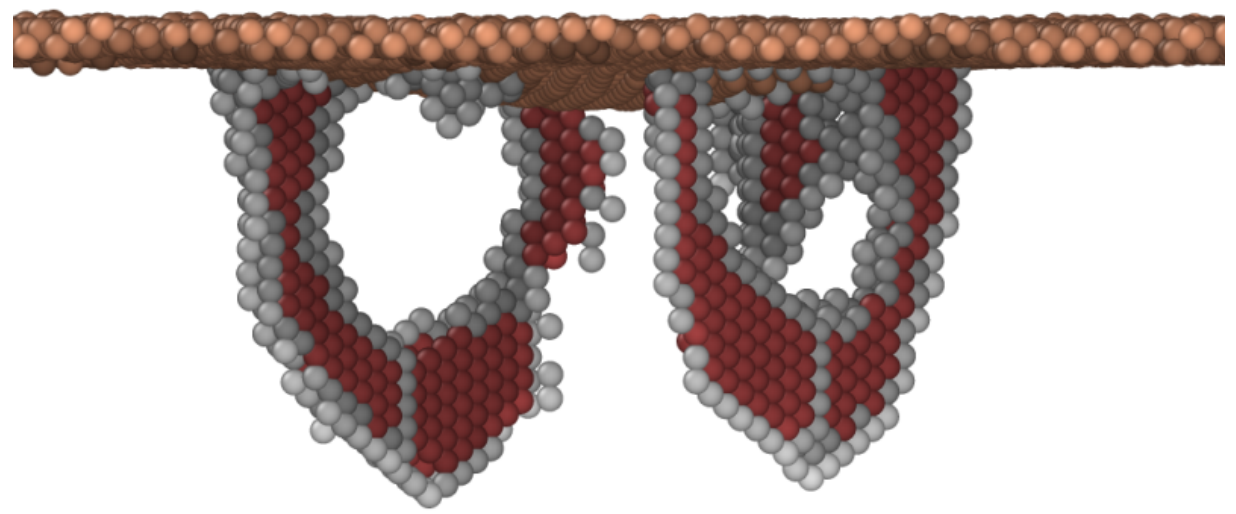

Abb. 5 Momentaufnahme der Bildung von Versetzungsringen in einer Molekulardynamiksimulation eines Nanoindentationsversuches. Zu sehen ist die Oberfläche (braun) eines (110)-orientierten Palladiumeinkristalls, welche mit einer $10 \mathrm{~nm}$ großen Nanoindenterspitze verformt wurde. Zur besseren Darstellung wurden alle Atome, welche dem idealen Gitter entsprechen, entfernt. Rot eingefärbte Atome haben eine (lokale) hexagonale Umgebung und entsprechen einem Stapelfehler in kfz-Gittern (es handelt sich daher um partielle Versetzungen), graue Atome konnten keinem definierten Gittertypen zugeordnet werden. Genauere Informationen zur Simulation sind im Kapitel 3.4 beschrieben.

\footnotetext{
${ }^{4}$ Es wurden folgende Werte verwendet: $G=44 \mathrm{GPa}[52],|b|=\left|\frac{a}{2}\langle 110\rangle\right|=0.275 \mathrm{~nm}[53]$ und $\gamma=180 \frac{\mathrm{mJ}}{\mathrm{m}^{2}}[54]$
} 


\section{Versetzungen im krz Gitter}

Die Beschreibung der atomaren Struktur von Versetzungen im krz Gitter ist komplex. Die Art der Versetzung sowie die Versetzungsbildung und ihre Bewegung hängen von der Temperatur [55], dem Metall [56] und der Konzentration von Fremdatomen [57] ab. Allgemein gilt, dass im krz Gitter der vorherrschende Versetzungstyp die Schraubenversetzung ist. Allerdings sind verschiedene konkrete Ausprägungen möglich [56, 58].

Hinsichtlich des Verhaltens von Versetzungen gibt es im wesentlichen für jedes krz Metall zwei Temperaturregionen [55]. Bei niedrigen Temperaturen bilden sich zwei Typen von Versetzungen aus. Diese sind (bei geringen mechanischen Spannungen) zum einen unbewegliche Schraubenversetzungen mit einem Burgersvektor von $b=\frac{a}{2}\langle 110\rangle$, zum anderen sehr bewegliche, gemischte Versetzungen. Der Unterschied in der Beweglichkeit der Versetzungen führt dazu, dass sich bei mechanischer Verformung im Metall Schraubenversetzungen akkumulieren und es so zu einer Verfestigung des Materials kommt. Zudem verlaufen Schraubenversetzungen sehr geradlinig, was damit erklärt wird, dass die Bildungsenergie von Kinken hoch ist, ihre Bewegung benötigt jedoch kaum Energie. Wenn Kinken gebildet werden, wandern diese entsprechend der mechanischen Verspannungen zu Positionen an denen sie annihilieren können. Dieses Verhalten kann auch in Molekulardynamiksimulationen [58, 59] gezeigt werden.

Bei höheren Temperaturen erhöht sich die Mobilität von reinen Schraubenversetzungen, wodurch das Verformungsverhalten ähnlich zu dem von kfz Metallen wird. Für gemischte Versetzungen ergibt sich jedoch der gegenläufige Effekt, da nun die Interaktion mit Fremdatomen entscheidender wird [60].

Um einen Eindruck von der Struktur von Versetzungen im krz Gitter zu erhalten, ist in Abbildung 6 ein Ausschnitt aus einer im Rahmen dieser Arbeit durchgeführten Molekulardynamiksimulation dargestellt. Hierbei handelt es sich um krz Eisen, welches durch Nanoindentation verformt wurde. Dabei sind nur Atome gezeigt, die nicht dem idealen Gitter entsprechen, wodurch die unter der Oberfläche entstandenen Versetzungen zu erkennen sind. Diese bilden, ausgehend von der Oberfläche, Versetzungsringe. Es handelt sich hierbei um gemischte Versetzungen, die sowohl Schraubenals auch Stufenabschnitte aufweisen.

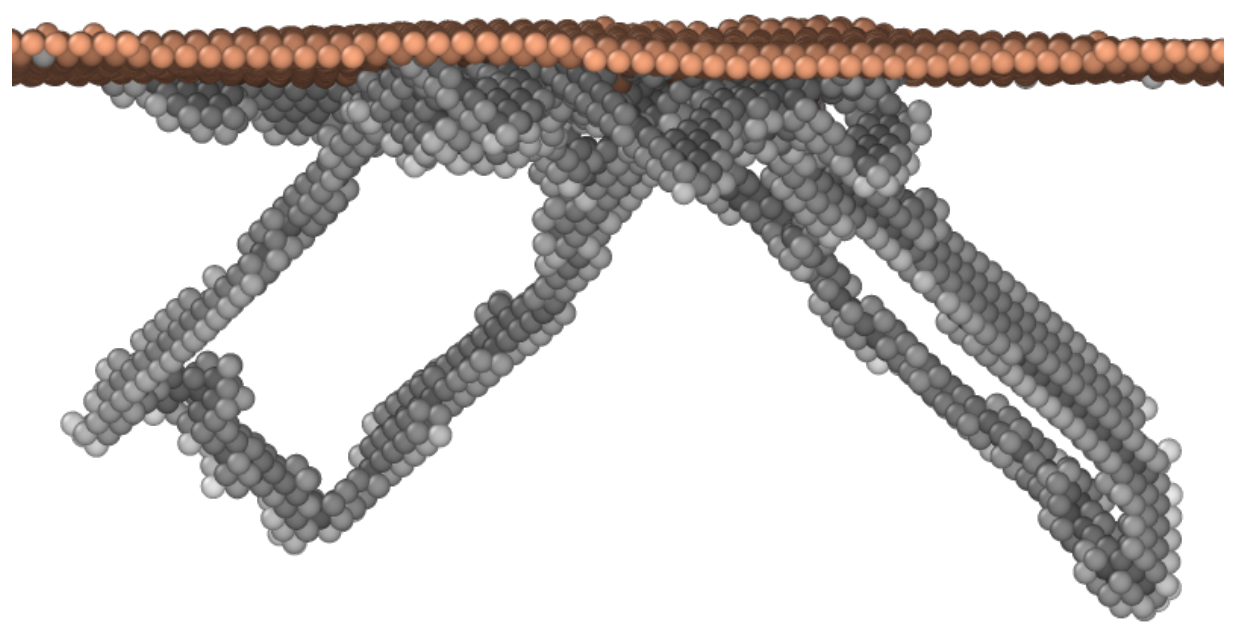

Abb. 6 Momentaufnahme der Bildung von Versetzungsringen in einer Molekulardynamiksimulation eines Nanoindentationsversuches. Zu sehen ist die Oberfläche

(braun) eines (100)-orientierten Eiseneinkristalls, welche mit einer $10 \mathrm{~nm}$ großen

Nanoindenterspitze verformt wurde. Zur besseren Darstellung wurden alle Atome, welche dem idealen Gitter entsprechen, entfernt. Grau eingefärbte Atome konnten keinem definierten

Gittertypen zugeordnet werden. In diesem Fall bilden diese Atome die Versetzungslinien aus.

Genauere Informationen zur Simulation sind im Kapitel 3.4 zu finden. 


\section{Interaktion von Wasserstoff mit Versetzungen}

Experimentelle Untersuchungen an Palladium $[61,62]$ zeigen, dass Proben mit einer hohen Versetzungsdichte bei gleichem chemischen Potential (Definition chemisches Potential siehe Kapitel 2.2) mehr Wasserstoff aufnehmen können. Anhand von Neutronenstreuexperimenten $[62,63]$ wurde gezeigt, dass sich der zusätzliche Wasserstoff im Bereich um Stufenversetzungen herum befindet. Je höher die Wasserstoffkonzentration im Metall ist, desto größer wird auch der Bereich um die Versetzungen, in dem eine lokal erhöhte Wasserstoffkonzentration gefunden wird. Diese lokalen Konzentrationen entsprechen dabei der Hydridphase von Palladium.

Stufenversetzungen werden im Gegensatz zu Schraubenversetzungen von langreichweitigen hydrostatischen Spannungen umgeben. Dies führt dazu, dass es vergrößerte Zwischengitterplätze gibt, vgl. Abbildung 7. Diese Plätze sind im Falle von Palladium energetisch günstiger als reguläre Zwischengitterplätze. Durch

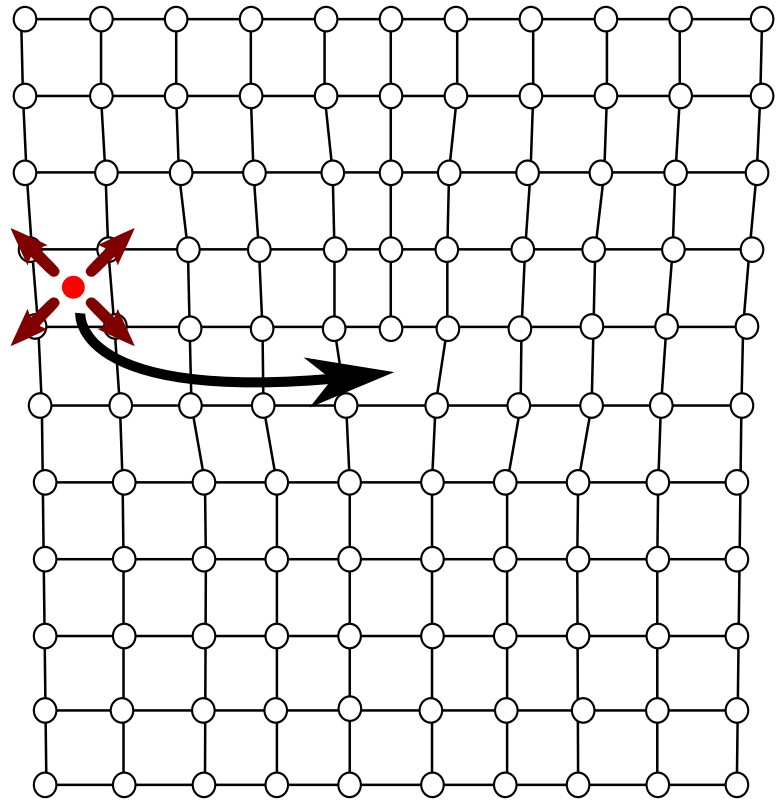

Abb. 7 Darstellung einer Stufenversetzung. Im Fall kleiner Wasserstoffkonzentrationen Diffundiert Wasserstoff zur Versetzung, da die Zwischengitterplätze geweitet sind. die Diffusion von Wasserstoff zu diesen Plätzen wird die vom Wasserstoff erzeugte Verspannung auf regulären Plätzen abgebaut. Andererseits werden die größeren Zwischengitterplätze durch Wasserstoff, vergleichbar zum Vorgang bei Leerstellen, stabilisiert. Dies bedeutet, dass die Linienenergie einer Stufenversetzung durch Wasserstoff reduziert werden kann. Die genauen thermodynamischen Zusammenhänge werden in Kapitel 2.3 dargestellt.

Die Auswirkungen von Wasserstoff auf Versetzungen können eindrucksvoll anhand von in-situ TEM Untersuchungen demonstriert werden [25]. In dieser Studie wurde eine Stahlprobe unter Zugspannung in einem sog. Environmental TEM (ETEM) untersucht. Wird eine Wasserstoffgasatmosphäre um die Probe erzeugt, bewegen sich Versetzungen und der Abstand zwischen aufgestauten Versetzungen verringert sich.

Dieses Verhalten wird durch das HELP-Modell (engl.: Hydrogen-Enhanced Localised Plasticity) beschrieben. Demnach schirmt Wasserstoff die Spannungsfelder von Versetzungen ab, wodurch deren Bewegung weniger durch andere Versetzungen oder Hindernisse behindert wird.

In Abbildung 8 wird schematisch die Auswirkung von gelöstem Wasserstoff auf Versetzungen und die dabei auftretenden mikrostrukturellen Vorgänge dargestellt. 


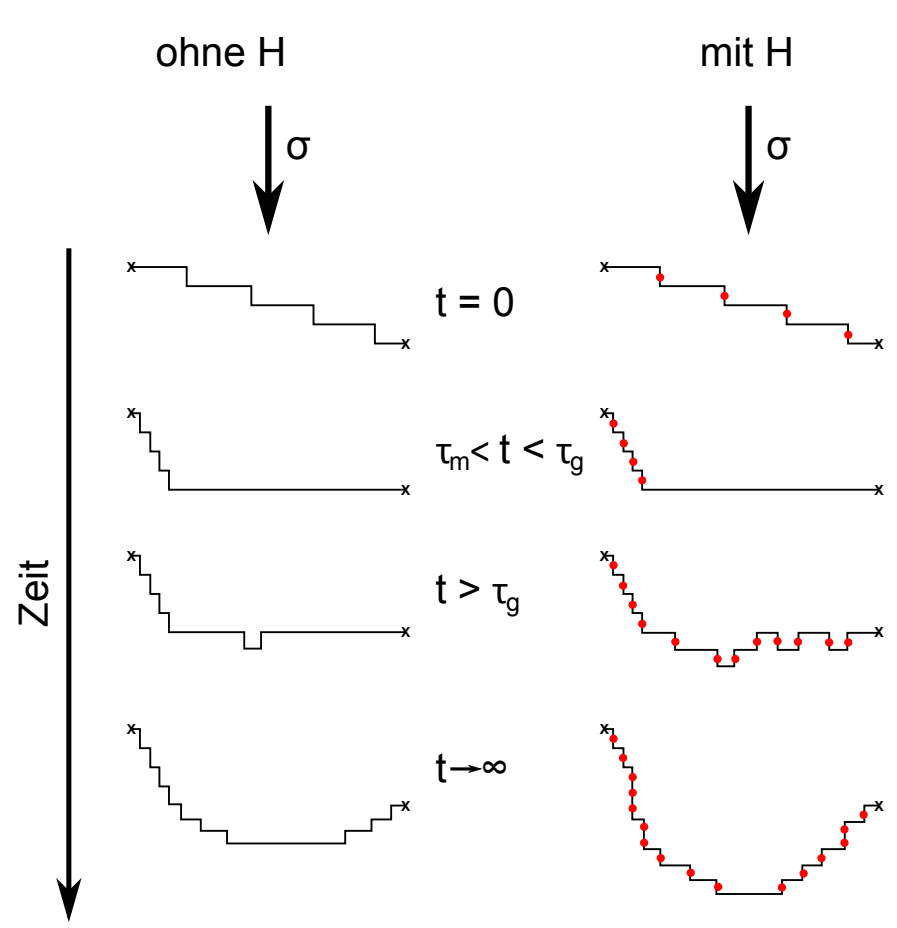

Abb. 8 Darstellung der Auswirkung von Wasserstoff auf die Versetzungsbewegung, nach [64]. Eine Versetzung, welche an zwei Punkten fixiert ist, muss im Regelfall sowohl Stufen- als auch Schraubenanteile besitzen. Wasserstoff im Metall lagert sich bevorzugt an Versetzungsteilstücken mit Stufencharakter (senkrechte Abschnitte) an. Wird eine Kraft $\sigma$ auf die Versetzung ausgeübt, bewegen sich zuerst die Kinken mit Stufencharakter. Da Wasserstoff sehr schnell im Metall diffundiert, wird die dazu benötigte Zeit $\tau_{m}$ kaum beeinflusst. Doppelkinkenpaare bilden sich thermodynamisch aktiviert in größeren Zeiträumen $\tau_{g}$. Wasserstoff reduziert die Linienenergie dieser (stufenartigen) Kinken, d. h. es werden mehr Doppelkinkenpaare gebildet und weniger Doppelkinkenpaare verschwinden wieder. Dadurch kann die Versetzung besser dem äußeren Spannungsfeld folgen, indem die Gesamtlänge größer wird.

Für eine Versetzung, deren Enden fixiert sind, folgt nach diesem Modell folgende Beschreibung der Bewegung [64, 65]: Liegen keine äußeren Spannungen vor, ist die Versetzungslinie so kurz wie möglich. Dabei muss die Versetzung jedoch dem energetischen Minimum (Peierls-Potential) (vgl. Abbildung 3) folgen. D. h. es gibt sowohl stufenartige Kinken als auch schraubenartige Versetzungsabschnitte. Da Wasserstoff bevorzugt die Linienenergie von Stufenversetzungen reduzieren kann, wird dieser sich dort anlagern.

Wird nun eine Scherkraft auf die Versetzung ausgeübt, wölbt sich diese aus, indem zunächst die Kinken sich bewegen. Die Zeit $\tau_{m}$, in der sich die Kinken bewegen, wird aufgrund der hohen Mobilität des Wasserstoffs in den meisten Metallen kaum beeinflusst.

Die weitere Bewegung der Versetzung beruht auf der Bildung von Doppelkinkenpaaren. Eine Versetzung an sich ist kein thermodynamisch stabiler Defekt, jedoch sind Doppelkinkenpaare Defekte entlang einer Versetzungslinie, welche, analog zu Leerstellen im Metall, entstehen und vergehen und somit eine Gleichgewichtskonzentration annehmen [51]. Wasserstoff stabilisiert diese Doppelkinkenpaare, wodurch die Gleichgewichtskonzentration steigt [64]. Umgekehrt kann dies als Reduktion der Zeit zum Bilden von Doppelkinkenpaare $\tau_{g}$ interpretiert werden.

Ausgehend von den Doppelkinkenpaaren können sich die Kinken wegbewegen, die Versetzung ist einen atomaren Abstand weiter gewandert.

Zusammengefasst bilden sich im Fall von Wasserstoff mehr Kinken im Vergleich zum wasserstofffreien Metall, da die Bildungsenergie der Kinken durch Wasserstoff reduziert ist. Somit kann eine Versetzung dem von außen angelegtem Spannungsfeld besser folgen. 


\subsection{Lösung von Wasserstoff in Metallen}

Die Lösbarkeit eines Stoffes in einem anderen wird zum einen durch geometrische Aspekte und zum anderen durch elektronische Beiträge beeinflusst. Wasserstoff lässt sich aufgrund seiner geringen Größe gut in vielen Metallen und Legierungen interstitiell lösen. Die Effekte einer WasserstoffBeladung sind für die einzelnen Metall-Wasserstoffsysteme sehr unterschiedlich. Die Beladung mit Wasserstoff führt bei allen bekannten Metall-Wasserstoffsystemen zu einer Volumenausdehnung der Elementarzelle [66]. Bei mittleren Konzentrationen können sich wasserstoffreiche Hydridphasen ausbilden. Dies hat zur Folge, dass im Material Spannungen aufgebaut werden, was zur Defektbildung führt. Bei vielen Systemen wird dabei die Gitterstruktur zerstört und in extremen Fällen kann das Material pulverisieren (z. B. zerfällt $\mathrm{LaNi}_{5}$ in Wasserstoffgas in kleinste Partikel [67, 68]).

Das Wasserstoffatom besetzt im Bereich kleiner Konzentrationen Zwischengitterplätze, also Tetraeder- oder Oktaederlücken [69].

Die Basis für eine theoretische Beschreibung der Effekte des gelösten Wasserstoffs auf elektronische Eigenschaften des Metalls ist das Bändermodell. Hierbei bilden die Orbitale der Metallatome quasikontinuierliche Energiebänder aus, die durch nicht mit Elektronen besetzte Energien voneinander getrennt sind (Bandlücke) [70]. Bei einfachen Modellen wird davon ausgegangen, dass die Bänderstruktur unverändert bleibt. Dabei wird der gelöste Wasserstoff als Ion behandelt, welches entweder ein Elektron an ein Band abgibt oder aufnimmt und somit die Fermienergie absenkt und somit einen Energiegewinn darstellt [19, 71, 72].

Im Rahmen quantenmechanischer Betrachtungen wird ein Fremdatom im periodischen Metallgitter gleichzeitig lokalisiert und als eine über alle gleichwertigen Plätze ausgedehnte Welle betrachtet, ähnlich der Bandstruktur von Elektronen in Metallen [38].

Bei Wasserstoff ist die Ausbildung von neuen Bändern zu vernachlässigen, da die Überlappungsintegrale der Wellenfunktionen zweier benachbarter Plätze zu klein sind [38]. Dies rechtfertigt die Vorstellung einzelner lokalisierter Wasserstoffionen im Metallgitter.

Zusammengefasst führt das Einbringen eines Wasserstoffelektrons in Energiebänder des Metalls zu einem Energiegewinn, gleichzeitig wird jedoch Arbeit aufgebracht, um das Gitter zu dehnen.

Aus thermodynamischer Sicht lässt sich die gute Löslichkeit von Wasserstoff mit der Reduzierung der Gibbschen Freien Enthalpie $G=H-T S$ erklären. Die Lösung von Wasserstoff in Metall beeinflusst zum einen die Entropie $S=S_{c o n f}+S_{v i b}$, welche sich in einen Konfigurationsanteil $S_{\text {conf }}$ (engl. configuration) und einen Anteil $S_{v i b}$ (engl. vibration) aufteilen lässt, wobei letzterer hauptsächlich aus Beiträgen von Schwingungen und elektronischen Einflüssen besteht. Zum anderen beeinflusst der Wasserstoff die Enthalpie $H$ durch elektronische Beiträge und durch Dehnung des Metallgitters.

Im Weiteren wird auf die theoretische Beschreibung eines defektfreien Metalls, welches einer Gasatmosphäre ausgesetzt ist, eingegangen [38, 73]. Hierbei wird zunächst das chemische Potential erläutert. Dieses beschreibt wie viel freie Energie pro Wasserstoffatom gewonnen/aufgewendet werden muss, wenn Wasserstoff in Metall gelöst wird. Anschließend wird der Zusammenhang zwischen dem von außen angelegten Wasserstoffgasdruck und der Wasserstoffkonzentration in der Probe hergeleitet.

Für kleine Wasserstoffkonzentrationen $c_{\mathrm{H}}=\frac{n_{\mathrm{H}}}{n_{\mathrm{M}}} \ll 1\left(n_{\mathrm{H}}\right.$ : gelöste Wasserstoffatome, $n_{\mathrm{M}}$ : Anzahl der Metallatome der Probe), d. h. im Einphasengebiet des jeweiligen Metall-Wasserstoffsystems, ergibt die Absorption und Desorption im Gleichgewicht folgende Gleichung:

$$
\mathrm{M}+\frac{n}{2} \mathrm{H}_{2} \rightleftharpoons \mathrm{MH}_{n}+n \Delta H
$$

$\Delta H$ ist die Lösungsenthalpie pro absorbiertem Wasserstoffatom. 
Des Weiteren müssen im thermodynamischen Gleichgewicht die chemischen Potentiale des Wasserstoffs im Metall $\left(\mu_{\mathrm{H}}\right)$ und des Wasserstoffs im Wasserstoffgas $\left(\mu_{\mathrm{H}_{2}}\right)$ gleich sein:

$$
\left.\mu_{\mathrm{H}}\right|_{c_{\mathrm{H}}, p, T}=\left.\frac{1}{2} \mu_{\mathrm{H}_{2}}\right|_{p, T}
$$

Da das chemische Potential die partielle Ableitung der Gibbschen Freien Enthalpie nach der Teilchenzahl ist, können die chemischen Potentiale wie folgt ausgedrückt werden:

$$
\begin{aligned}
\mu_{\mathrm{H}_{2}} & =\frac{\partial H}{\partial n_{\mathrm{H}_{2}}}-T \frac{\partial S}{\partial n_{\mathrm{H}_{2}}} \\
\mu_{\mathrm{H}} & =\frac{\partial H}{\partial n_{\mathrm{H}}}-T \frac{\partial S}{\partial n_{\mathrm{H}}}
\end{aligned}
$$

Wird das Wasserstoffgas als ideales Gas aufgefasst, d. h. dass die Enthalpie nicht von der Teilchenzahl abhängt und dass sich die Entropie durch $S=\left.S\right|_{p_{0}}+N k_{B} \ln \left(\frac{p_{0}}{p}\right)$ beschreiben lässt, kann das chemische Potential des Wasserstoffgases wie folgt dargestellt werden:

$$
\mu_{\mathrm{H}_{2}}=\mu_{\mathrm{H}_{2}, 0}+k_{B} T \ln \left(\frac{p}{p_{0}}\right)
$$

Hierbei bezeichnet $\mu_{\mathrm{H}_{2}, 0}$ das chemische Potential bei einem Druck von $p_{0}=1 \mathrm{Atm}=101.325 \mathrm{~Pa}$ bei der Temperatur $T$.

Im Folgenden wird das chemische Potential des Wasserstoffs in Metall als Funktion der Wasserstoffkonzentration $c_{\mathrm{H}}$ dargestellt. Dazu wird die Konfigurationsentropie $S_{\text {conf }}$ nach Boltzmann verwendet:

$$
\begin{aligned}
S_{\text {conf }} & =k_{B} T \ln \left(\frac{r n_{\mathrm{M}} !}{n_{\mathrm{H}} !\left(r n_{\mathrm{M}}-n_{\mathrm{H}}\right) !}\right) \\
& \approx k_{B} T\left[r n_{\mathrm{M}} \ln \left(\frac{r n_{\mathrm{M}}}{r n_{\mathrm{M}}-n_{\mathrm{H}}}\right)-n_{\mathrm{H}} \ln \left(\frac{n_{\mathrm{H}}}{r n_{\mathrm{M}}-n_{\mathrm{H}}}\right)\right]
\end{aligned}
$$

Hierbei verteilen sich $n_{\mathrm{H}}$ Wasserstoffatome auf $r n_{\mathrm{M}}$ Zwischengitterplätze ( $r$ : Anzahl der Zwischengitterplätze pro Metallatom). Um Gleichung $2.13 \mathrm{zu}$ erhalten, wurde die Stirling Formel $(\ln (x !) \approx x \ln (x)-x)$ angewendet. Somit ergibt sich für die partielle Ableitung nach der Wasserstoffatomanzahl:

$$
\begin{aligned}
\frac{\partial S_{\text {conf }}}{\partial n_{\mathrm{H}}} & =-k_{B} T \ln \left(\frac{n_{\mathrm{H}}}{r n_{\mathrm{M}}-n_{\mathrm{H}}}\right) \\
& =-k_{B} T \ln \left(\frac{c_{\mathrm{H}}}{r-c_{\mathrm{H}}}\right)
\end{aligned}
$$

Unter der Bedingung 2.8 können beide Formen des chemischen Potentials (Gleichung 2.10 und 2.11) miteinander verknüpft werden [38, 73], es ergibt sich das Sievertssche Gesetz [74], welches den Zusammenhang zwischen Wasserstoffgasdruck und Wasserstoffkonzentration im Metall darstellt:

$$
\frac{c_{\mathrm{H}}}{r-c_{\mathrm{H}}}=K \sqrt{\frac{p}{p_{0}}}
$$

Hierbei ist $K$ ein Parameter, welcher von der Temperatur abhängt.

Im Folgenden werden zwei Modelle, welche die Lösung von Wasserstoff in Metall aus der Gasphase bzw. aus einem Elektrolyten beschreiben, vorgestellt. 


\subsection{1 Übergang von Wasserstoff aus der Gasphase ins Metall}

In diesem Abschnitt wird der Vorgang der Lösung von Wasserstoff in Metall beschrieben, die thermodynamischen Zusammenhänge sind in Kapitel 2.2 bereits beschrieben worden.

Die Lösung von Wasserstoff ins Metall erfolgt in mehreren Schritten [75-80]: Zunächst können Wasserstoffmoleküle durch Van-der-Waals Kräfte an der Oberfläche gehalten werden, dies wird Physisorption genannt. Dieser Vorgang ist vollständig reversibel.

Wird das Molekül näher zur Oberfläche gebracht, kann es dissoziieren und die Wasserstoffatome gehen eine chemische Verbindung mit Metallatomen der Oberfläche ein. Dieser Prozess ist typischerweise langsam im Gegensatz zu den anderen Lösungsschritten und benötigt eine Aktivierungsenergie.

Der letzte Schritt ist die Lösung des Wasserstoffatoms im Metallgitter. Hierbei nimmt es typischerweise Zwischengitterplätze ein. Eine Darstellung der beteiligten Beiträge zur potentiellen Energie ist in Abbildung 9 dargestellt.

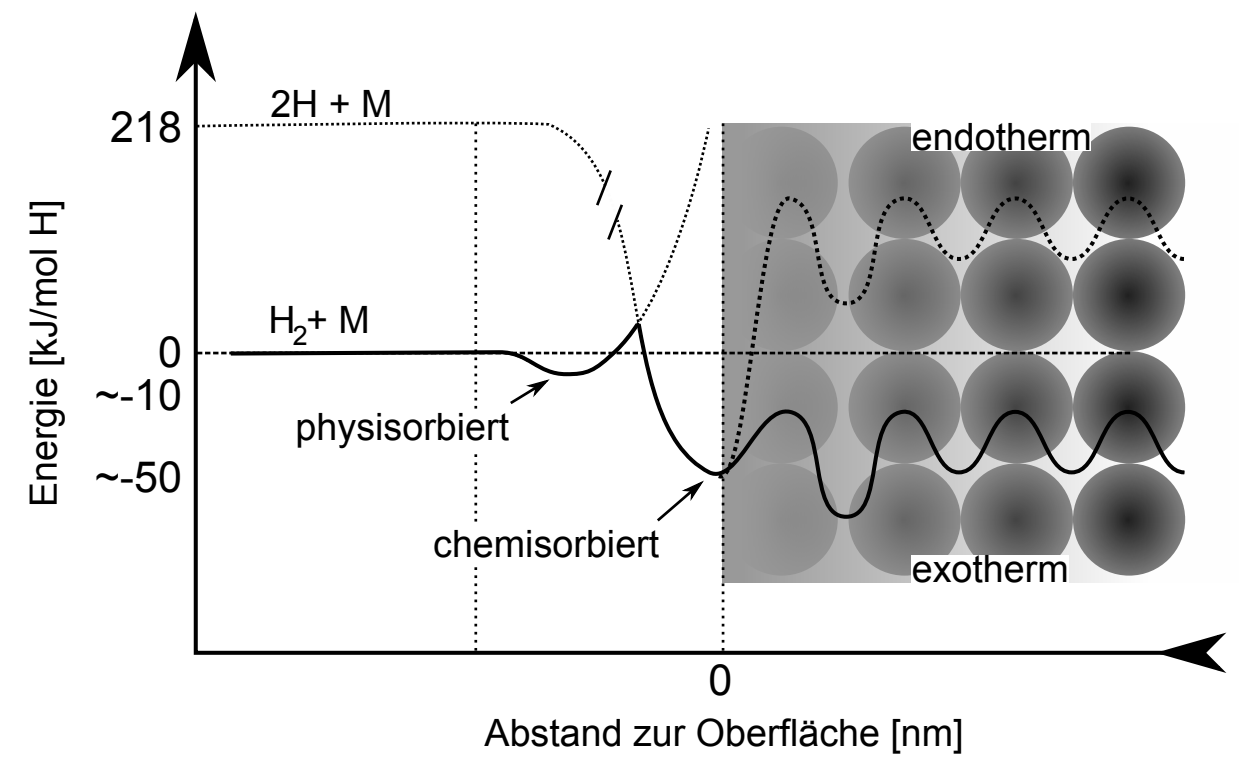

Abb. 9 Schematische Darstellung der Beiträge zur potentiellen Energie von Wasserstoffatomen an einer Metalloberfläche, zur Darstellung der Lösung von Wasserstoff in Metall [75-79]. 


\subsection{2 Übergang von Wasserstoff in Metall bei elektrolytischer Beladung}

Die Gesamtgleichung der Lösung von Wasserstoff aus einem Elektrolyten in ein Metall wird durch die sog. HER-Reaktionsgleichung (engl.: Hydrogen Evolution Reaction) beschrieben [81]:

$$
\begin{aligned}
& 2 \mathrm{H}_{3} \mathrm{O}^{+}+2 \mathrm{e}^{-} \longrightarrow \mathrm{H}_{2}+2 \mathrm{H}_{2} \mathrm{O} \text { in sauren Lösungen } \\
& 2 \mathrm{H}_{2} \mathrm{O}+2 \mathrm{e}^{-} \longrightarrow \mathrm{H}_{2}+2 \mathrm{OH}^{-} \text {in basischen Lösungen }
\end{aligned}
$$

Die eigentliche Reaktion läuft jedoch in mehreren Einzelschritten ab. Zunächst wird ein Wasserstoffatom an der Oberfläche gebunden:

$$
\begin{aligned}
\mathrm{M}+\mathrm{H}_{3} \mathrm{O}^{+}+\mathrm{e}^{-} & \rightarrow \mathrm{MH}_{\text {ads }}+\mathrm{H}_{2} \mathrm{O} \quad \text { in sauren Lösungen } \\
\mathrm{M}+\mathrm{H}_{2} \mathrm{O}+\mathrm{e}^{-} & \longrightarrow \mathrm{MH}_{\text {ads }}+\mathrm{OH}^{-} \quad \text { in basischen Lösungen }
\end{aligned}
$$

Hierbei beschreibt $\mathrm{MH}_{\text {ads }}$ ein an der Oberfläche des Metalls adsorbiertes Wasserstoffatom. Diesen adsorbierten Wasserstoffatomen stehen nun mehrere Reaktionsmöglichkeiten offen.

Durch Diffusion an der Oberfläche können zwei adsorbierte Wasserstoffatome aufeinander treffen und zu einem Wasserstoffmolekül reagieren:

$$
2 \mathrm{MH}_{\mathrm{ads}} \longrightarrow{ }_{2} \mathrm{M}+\mathrm{H}_{2}
$$

Eine andere Möglichkeit ist, dass die adsorbierten Wasserstoffatome mit Wasserstoffatomen aus der Lösung reagieren:

$$
\begin{gathered}
\mathrm{MH}_{\mathrm{ads}}+\mathrm{H}_{3} \mathrm{O}^{+}+\mathrm{e}^{-} \longrightarrow \mathrm{M}+\mathrm{H}_{2} \mathrm{O}+\mathrm{H}_{2} \quad \text { in sauren Lösungen } \\
\mathrm{MH}_{\text {ads }}+\mathrm{H}_{2} \mathrm{O}+\mathrm{e}^{-} \longrightarrow \mathrm{M}+\mathrm{OH}^{-}+\mathrm{H}_{2} \quad \text { in basischen Lösungen }
\end{gathered}
$$

Einige Metalle können Wasserstoff aufnehmen. Somit steht dem adsorbierten Wasserstoffatom ein weiterer Reaktionsweg offen. Aus dem adsorbierten Zustand kann das Wasserstoffatom auf einen Zwischengitterplatz unterhalb der Oberfläche wechseln $\left(\mathrm{MH}_{\mathrm{abs}}\right)$ und von dort aus ins Metall diffundieren [75].

$$
\mathrm{MH}_{\mathrm{ads}} \rightleftharpoons \mathrm{MH}_{\mathrm{abs}}
$$

Welcher der Reaktionswege benutzt wird, hängt neben der Wahl des Elektrolyten und des Metalls (bzw. der Legierung) z. B. von den Oberflächeneigenschaften, der Stromdichte, dem Elektrodenpotential, der Temperatur, dem Druck und vielem mehr ab. Für die Aufnahme von Wasserstoff in Metall ist vor allem der Bedeckungsgrad mit adsorbierten Atomen von Bedeutung [75]. 


\subsubsection{Diffusion von Wasserstoff in Metallen}

Diffusion ist ein Prozess, der es ermöglicht, ein System in das thermodynamische Gleichgewicht zu überführen. Treibende Kraft dafür ist der mögliche Gewinn an freier Enthalpie, was dem chemischen Potential $\mu$ der diffundierenden Teilchen entspricht.

Der Unterschied im lokalem chemischen Potential gibt an, wie viel Energie frei wird, wenn ein Atom seinen Ort wechselt. Dabei hängt der lokale Wert des chemischen Potentials nicht nur von der chemischen Umgebung ab; darüber hinaus können z. B. mechanische Spannungen [82], Temperaturunterschiede oder elektrische Felder [83] das chemische Potential beeinflussen.

Beruht die Diffusion nur auf dem Vorhandensein von Konzentrationsgefällen und liegen kleine Konzentrationen vor, kann die Diffusion einer Teilchensorte in einem anderen Material mit dem ersten Fickschen Gesetz beschreiben werden [45]. Für eindimensionale Diffusion in Richtung $x$ ergibt sich:

$$
J=-\widetilde{D} \frac{\partial c}{\partial x}
$$

Der Teilchenstrom $J$ ergibt sich aus einem örtlichen Gradienten der Konzentration $c$, welche proportional zum chemischen Diffusionskoeffizienten $\widetilde{D}$ ist.

Durch den Bezug auf das chemische Potential $\mu$ lässt sich diese Gleichung auf beliebige Konzentrationen verallgemeinern [45]:

$$
J=-M \frac{\partial \mu}{\partial x}
$$

Hierbei ist $M$ ein Parameter, welcher sich aus der Beweglichkeit $B$ und der Konzentration zusammensetzt, $M=B c$. Um die beiden Gleichungen miteinander zu verknüpfen, wird die Abhängigkeit des Diffusionskoeffizienten von der Konzentration benötigt. Werden die beiden Gleichungen gleichgesetzt, ergibt sich:

$$
\begin{aligned}
B c \frac{\partial \mu}{\partial x} & =\widetilde{D} \frac{\partial c}{\partial x} \\
B \frac{\partial \mu}{\partial x} & =\widetilde{D} \frac{1}{c} \frac{\partial c}{\partial x}=\widetilde{D} \frac{\partial \ln (c)}{\partial x} \\
\Rightarrow \widetilde{D} & =B \frac{\partial \mu}{\partial \ln (c)}
\end{aligned}
$$

Das chemische Potential in Abhängigkeit der Konzentration ist gegeben durch:

$$
\mu=\mu_{0}+R T \ln \left(\gamma^{*} c\right),
$$

mit $R$ der universellen Gaskonstanten und $T$ der Temperatur. $\mu_{0}$ beinhaltet alle Beträge zum chemischen Potential, die über das Material nicht variieren und somit keine Triebkraft für diffundierende Teilchen darstellen. $\gamma^{*}$ ist der Aktivitätskoeffizient und ist im Allgemeinen eine Funktion der Konzentration. Damit ergibt sich:

$$
\begin{aligned}
\frac{\partial \mu}{\partial \ln (c)} & =R T\left(1+\frac{\partial \ln \left(\gamma^{*}\right)}{\partial \ln (c)}\right) \\
\Rightarrow \widetilde{D} & =B R T\left(1+\frac{\partial \ln \left(\gamma^{*}\right)}{\partial \ln (c)}\right) \\
& \equiv D_{0}\left(1+\frac{\partial \ln \left(\gamma^{*}\right)}{\partial \ln (c)}\right)
\end{aligned}
$$

Der Ausdruck in Klammern wird thermodynamischer Faktor genannt. Für kleine Konzentrationen ist $\gamma^{*}(c)=\operatorname{const}(c)$, d. h. $\widetilde{D}=D_{0}$. Der chemische Diffusionskoeffizient $\widetilde{D}$ bei verschwindend kleiner Konzentration wird auch als Selbstdiffusionskoeffizient $D_{0}$ bezeichnet. 
Bisher wurde nur die Auswirkung eines Konzentrationsungleichgewichts auf die Diffusion beschrieben. Werden andere Einflüsse zusätzlich betrachtet, ergibt sich das chemische Potential als Summe der Einzelbeiträge [84].

Das chemische Potential, was sich aufgrund von mechanischen Spannungen ergibt, ist gegeben durch [85]:

$$
\mu=\mu_{0}+\omega+\sigma \nu
$$

Wenn eine Spannung $\sigma$ vorliegt, ergibt sich das chemische Potential aus der molaren Dehnungsenergie $\omega$ und dem molaren Volumen im verspannten Zustand $\nu$.

Wasserstoff wird also entlang des mechanischen Spannungsfelds, entgegen der Spannung diffundieren. Im Fall von Stufenversetzungen bedeutet dies, dass Wasserstoff in den unter Zugspannung stehenden Bereich unterhalb des Versetzungskerns diffundiert (vgl. Abbildung 10).
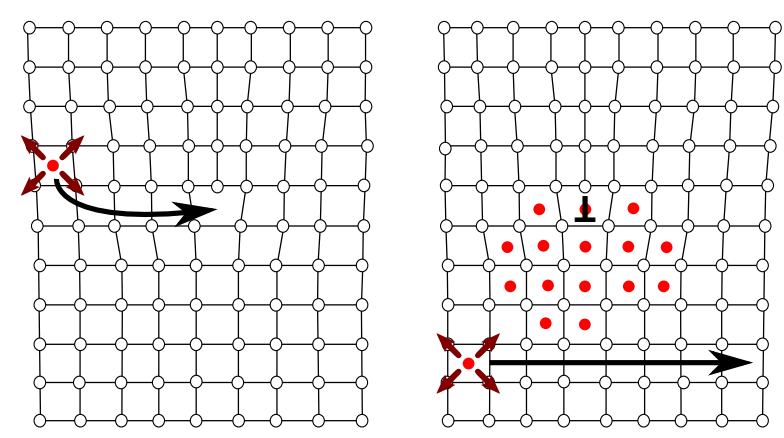

Abb. 10 Diffusion eines Wasserstoffatoms im Spannungsfeld einer Versetzung. Im Fall kleiner Wasserstoffkonzentrationen (links) diffundiert Wasserstoff zu den Versetzungen und hält sich dort länger aufgrund der größeren $Z_{w i-}$ schengitterplätze auf; die Diffusion ist verlangsamt. Bei höheren Konzentrationen (rechts) sind die verspannten $Z$ wischengitterplätze belegt und weiterer Wasserstoff wird kaum beeinflusst von den Spannungsfeldern der Versetzungen.

Zusammengefasst ergibt sich folgendes Bild: Wasserstoff wird sich im Metall homogen verteilen. Liegt jedoch ein Spannungsfeld vor, wird die Diffusion von Wasserstoff in die gedehnte Richtung begünstigt. Dies führt zur lokalen Erhöhung der Konzentration im Spannungsfeld von z. B. Versetzungen. Die lokale Konzentration an einer Versetzung ergibt sich aus dem Gleichgewicht zwischen der Reduzierung des chemischen Potentials aufgrund des Spannungsfelds und dem chemischen Potential, welches sich aufgrund der Erhöhung der lokalen Konzentration ergibt.

Im Fall einer versetzungsreichen Probe und kleinen Konzentrationen ergibt sich das Diffusionsverhalten als Überlagerung von Einflüssen der Konzentrationsunterschiede und mechanischen Spannungen der Versetzungen. Sind die Spannungsfelder der Versetzungen hinreichend mit den diffundierenden Teilchen besetzt, tragen diese nicht mehr zum chemischen Potential bei. Dies bedeutet für die Diffusion, dass bei kleinen Konzentrationen die Diffusion durch die Spannungsfelder der Versetzungen gestört wird und bei höheren Konzentrationen sich ein Verhalten ausbildet, welches dem eines versetzungsfreien Materials entspricht. 


\subsection{Thermodynamik von Defekten und gelösten Atomen Das Defactant-Konzept}

Da alle Arten von Kristallbaufehlern von einem Spannungsfeld umgeben sind und dies auch für gelöste Fremdatome gilt, wird ersichtlich, dass Fremdatome und andere Kristallbaufehler miteinander interagieren.

In vielen Fällen diffundieren Fremdatome zu den Kristallbaufehlern. Dies führt zum Abbau von Spannungen im Material und kann somit die Gesamtenergie reduzieren.

Ein lange untersuchtes Beispiel ist Kohlenstoff in Eisen. Kohlenstoff diffundiert in die Umgebung von Versetzungen [86]. Wird das Material einer mechanischen Spannung ausgesetzt, wird in diesem Fall eine hohe Spannung benötigt, um das Eisen zu verformen. Haben sich jedoch die Versetzungen vom Kohlenstoff entfernt, kann das Eisen mit geringerer Spannung weiter verformt werden. Wird das Eisen geglüht, so dass der Kohlenstoff wieder zu den Versetzungen diffundieren kann, kehrt die erhöhte Festigkeit zurück. Dies bedeutet, dass Versetzungen durch Kohlenstoff immobilisiert werden. Neuere Untersuchungen von Chen et. al. [37] zeigen, dass Kohlenstoff in Eisen sich auch an Korngrenzen akkumuliert. Hier zeigt sich sogar, dass nicht nur Korngrenzen stabilisiert werden, sondern auch, dass die Gesamtfläche der Korngrenzen durch das Vorhandensein von Kohlenstoff vergrößert werden kann.

Ein weiteres Beispiel ist Bismut in Kupfer [87]. Bei erhöhten Temperaturen (1223 K) diffundiert Bismut an die Korngrenzen. An diesen bilden sich nun facettierte Hohlräume, deren Oberflächen mit Bismut bedeckt sind. Dieses Beispiel zeigt, dass die Stabilisierung bzw. die Energiereduzierung des gesamten Material soweit gehen kann, dass neue Kristallbaufehler erzeugt werden können.

Die thermodynamische Beschreibung der Interaktion von gelösten Fremdatomen im Material mit Kristallbaufehlern erfolgt im Rahmen des Defactant-Konzepts und soll im Folgenden kurz vorgestellt werden. Anschließend wird auf die atomaren Vorgänge bei der Interaktion von Versetzungen mit Fremdatomen eingegangen.

\section{Das Defactant-Konzept}

Um die Entstehung von Defekten in einen thermodynamischen Kontext zu erklären, kann die Helmholtz-Freie-Energie betrachtet werden. Diese setzt sich aus der inneren Energie $U$ und dem Entropieanteil TS zusammen. Dabei werden zwei Entropieanteile unterschieden, einen Vibrationsanteil $S_{v i b}$ und einen der die Konfigurationsentropie $S_{c o n f}$ [45]. Die Helmholtz-Freie-Energie ergibt sich danach zu:

$$
F=U-T\left(S_{\text {conf }}+S_{v i b}\right)
$$

Wird ein Defekt gebildet, so steigt die innere Energie um die Defektenergie $\gamma$ an. Des Weiteren steigt auch die Konfigurationsentropie [45]. Für Leerstellen sind diese beiden Beiträge in der gleichen Größenordnung, dies bedeutet, dass im Gleichgewicht Leerstellen vorhanden sein müssen, deren Gleichgewichtskonzentration konstant ist [45] (siehe auch Kapitel 2.1).

Für andere Defekte (Bspw. Versetzungen oder Korngrenzen) ist die Änderung in der Konfigurationsentropie klein gegenüber der Defektenergie, daher sind diese Defekte nicht thermodynamisch stabil.

Sind im Material Fremdatome gelöst, ändert sich diese Betrachtung. Gelöste Fremdatome ändern die innere Energie, indem das Material verspannt wird und die lokale Elektronendichte beeinflusst wird [19, 88-90].

Ein Kristallbaufehler beeinflusst auch diese Anteile der inneren Energie [45, 91-93], was nahelegt, dass Fremdatome mit Defekten interagieren müssen.

Dieses Zusammenspiel wird im Defactant-Konzept beschrieben [27-30].

Dabei stellt das Defactant-Konzept eine Weiterentwicklung der Theorie zur Beschreibung der In- 
teraktion von gelösten Stoffen mit Oberflächen dar, welche von Gibbs [94] beschrieben wurde. Werden bspw. Lipide ${ }^{5}$ in Wasser gelöst, bildet sich an der Oberfläche Schaum, d. h. die Oberfläche wird vergrößert und mehr Lipide finden eine energetisch günstige Position. Gibbs hat diese Systeme wie folgt beschrieben [94]:

$$
\begin{aligned}
\mathrm{d} \gamma & =-\Gamma_{A} \mathrm{~d} \mu_{A} \\
\Rightarrow-\Gamma_{A} & =\left.\frac{\partial \gamma}{\partial \mu_{A}}\right|_{T, V, a, n_{B}}
\end{aligned}
$$

Wenn sich gelöste Stoffe $A$ an einer Oberfläche $a$ anlagern und dabei eine Stoffmenge $\Gamma_{A}$, Excess genannt, annehmen, reduziert sich die Oberflächenenergie $\gamma$ je nach der angebotenen Stoffkonzentration oder allgemeiner mit dem chemischen Potential $\mu_{A}$, unter der Voraussetzung, dass die Anzahl der Wirtsatome $n_{B}$ unverändert bleibt.

Zur Verallgemeinerung auf Kristallbaufehler müssen die beteiligten Parameter so definiert werden, dass diese durch messbare Größen und mit Hilfe von bekannten Parametern dargestellt werden können.

Daher wird für Versetzungen der Excess $\Gamma_{A}^{D}$ (engl.: Dislocation) durch die Anzahl der Fremdatome $n_{A}$ pro Längeneinheit $l$ ermittelt; für Leerstellen wird der Excess $\Gamma_{A}^{V}$ (engl.: Vacancy) durch die Anzahl der Fremdatome auf die Anzahl der Leerstellen ermittelt:

$$
\begin{aligned}
\Gamma_{A}^{D} & =\left.\frac{\partial n_{A}}{\partial l}\right|_{T, V, \mu_{A}, n_{B}} \\
\Gamma_{A}^{V} & =\left.\frac{\partial n_{A}}{\partial n_{V}}\right|_{T, V, \mu_{A}, n_{B}}
\end{aligned}
$$

Die Oberflächenenergie wird ersetzt durch die Linienenergie $\gamma_{D}$ bei Versetzungen bzw. durch die Bildungsenergie $F_{v}$ bei Leerstellen, somit kann die Gleichung 2.33 wie folgt für Versetzungen bzw. für Leerstellen dargestellt werden:

$$
\begin{aligned}
& -\Gamma_{A}^{D}=\left.\frac{\partial \gamma_{D}}{\partial \mu_{A}}\right|_{T, V, l, n_{B}} \\
& -\Gamma_{A}^{V}=\left.\frac{\partial F_{V}}{\partial \mu_{A}}\right|_{T, V, n_{V}, n_{B}}
\end{aligned}
$$

Um quantitative Aussagen z. B. über die Linienenergie einer Versetzung in Abhängigkeit der Konzentration von gelösten Fremdatomen zu erhalten, muss die Abhängigkeit des Excess vom chemischen Potential bekannt sein. Daher setzen hier Untersuchungen an [40, 95-97].

Qualitativ lässt sich die Defektenergie als Funktion des chemischen Potentials wie folgt erklären (vgl. Abbildung 11): Bei kleinen chemischen Potentialen, d. h. bei kleinen Konzentrationen befinden sich nur wenige Fremdatome an den Defekten. Daher wird die Defektenergie kaum verändert. Wird das chemische Potential der Fremdatome erhöht, lagern sich Fremdatome an den Defekten an. Je nach Abhängigkeit des Excesses vom chemischen Potential ergibt sich ein Gleichgewichtsexcess $\Gamma_{A}^{\text {sat }}$ (Verlauf 1 in Abbildung 11) oder der Excess nimmt mit chemischen Potential weiter zu (Verlauf 2 in Abbildung 11). Dies hat nach Gleichung 2.33 zur Folge, dass die Defektenergie abnimmt.

Wird das chemische Potential weiter erhöht, kommt es, falls die Defektenergie Verlauf 1 oder 2 in Abbildung 11 folgt, dazu dass die Defektbildungsenergie auf Null absinkt. Dies bedeutet, dass neue Defekte entstehen können, welche wiederum Fremdatome aufnehmen und somit das chemische Potential wieder herabsetzen. Es werden beim Erreichen von $\gamma=0$ nicht beliebig neue Defekte entstehen, sondern nur so viele, sodass das chemische Potential wieder entsprechende Werte unterschreitet und somit wieder gilt $\gamma>0$. So gesehen beschreibt dieser Vorgang eine

\footnotetext{
${ }^{5}$ Lipide sind Moleküle, welche aus einen hydrophilen und einen hydrophoben Anteil bestehen und daher ihre energetisch günstigste Position finden, wenn sich ein Teil im Wasser und der andere Teil außerhalb befindet.
} 
Phasenumwandlung von defektfreiem Material hin zu defektreichem Material, bei der, wie bei einer Phasenumwandlung 1. Ordnung, das chemische Potential bei Erhöhung der Konzentration konstant bleibt. Gibt es jedoch einen konkurrierenden Phasenübergang, bspw. die Bildung einer Hydridphase im Fall von Metall-Wasserstoffsystemen, bleibt das chemische Potential aufgrund der Phasenumwandlung konstant und die Defektenergie bleibt konstant, es ergibt sich Verlauf 3 in Abbildung 11.

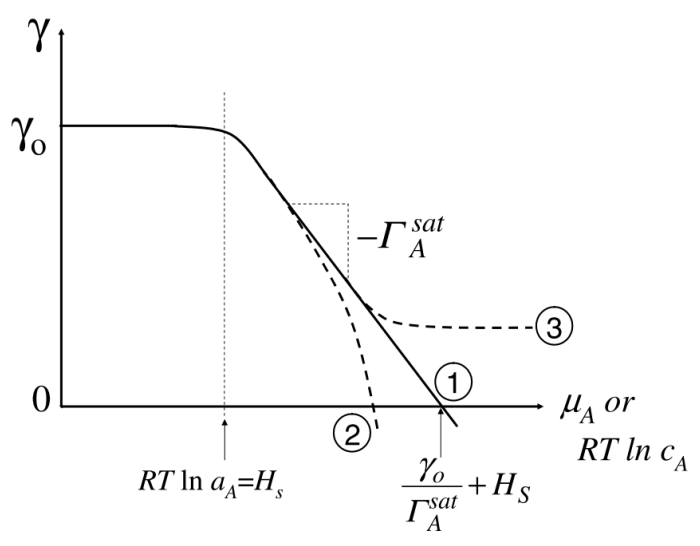

Abb. 11 Schematische Abhängigkeit der Defektenergie $\gamma$ vom chemischen Potential $\mu_{A}$. Bei kleinen chemischen Potentialen segregieren nur wenige Atome an Defekten, der Excess $\Gamma$ ist klein. Daher wird $\gamma$ kaum beeinflusst. Wird $\mu_{A}$ erhöht ergibt sich je nach der Abhängigkeit $\Gamma$ auf $\mu_{A}$ eine Sättigung des Excesses $\Gamma_{A}^{\text {sat }}$ (Verlauf 1) oder $\Gamma$ nimmt mit $\mu_{A}$ zu (Verlauf 2). Wird $\gamma=0$ erreicht bilden sich neue Defekte, solange freie Fremdatome vorhanden sind und somit $\mu_{A}$ genügend groß ist. Gibt es einen konkurrierenden Phasenübergang bleibt $\mu_{A}$ und somit $\gamma$ bei Erhöhung der Konzentration konstant, es ergibt sich Verlauf 3. Abbildung übernommen aus [27].

Zusammengefasst wird die Gesamtenergie eines Material mit Kristallbaufehlern reduziert, wenn Fremdatome sich an diesen anlagern und somit die Defektenergie reduzieren. Der Grund dafür setzt sich zusammen aus dem Abbau von mechanischen Spannungen im Material [18, 40, 44] und der Reduzierung der lokalen Elektronendichte [98-101], wenn Fremdatome an einen Kristallbaufehler diffundieren. 


\subsection{Verwendete Metall-Wasserstoffsysteme}

Für die Untersuchungen dieser Arbeit wurden zwei verschiedene Metalle ausgewählt, Palladium und Vanadium. Beide Metalle können mit einer im Vergleich zu anderen Metallen großen Menge an Wasserstoff beladen werden, ohne dass eine Hydridphase gebildet wird. Da Palladium ein kfz Metall und und Vanadium ein krz Metall ist, erlauben die Untersuchungen einen Überblick über die prinzipiellen Vorgänge, die durch Wasserstoff in Metallen ausgelöst werden. Ein weiterer Aspekt ist die Unterscheidung von Vorgängen, die durch Wasserstoff im Allgemeinen hervorgerufen werden, zu materialspezifischen Vorgängen.

Im Folgenden werden die einzelnen Metall-Wasserstoffsysteme vorgestellt.

\section{Das Palladium-Wasserstoffsystem}

Da Palladium ein Edelmetall ist, kann der Einfluss von Wasserstoff auf die plastische Verformung untersucht werden, ohne dass andere Effekte wie z. B. eine Oxidschicht oder eine fehlende Beständigkeit gegen Säuren, die Messung beeinflussen.

Palladium erlaubt es im Vergleich zu anderen Metallen relativ große Mengen Wasserstoff bei Raumtemperatur ${ }^{6}$ zu lösen, ohne dass es zur Bildung einer neuen wasserstoffreichen Phase kommt, siehe Abbildung 12.

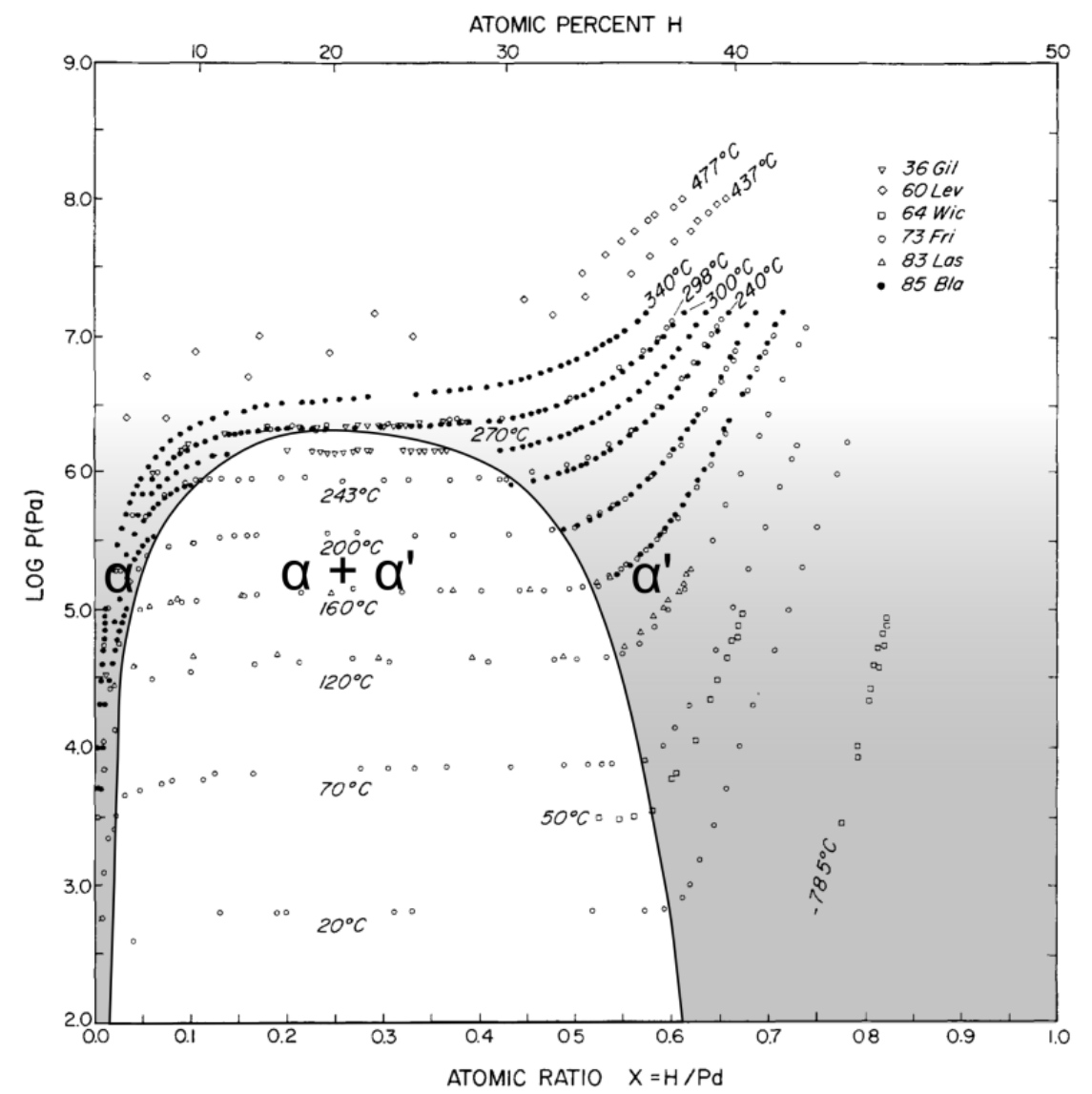

Abb. 12 Druck-Konzentrationsphasendiagramm des Palladium-Wasserstoffsystems [53]. Bei Raumtemperatur existieren zwei Phasen: Bei niedrigen Konzentrationen ${ }^{6}$ liegt die $\alpha$-Phase vor, ab $0.58 \mathrm{H} / \mathrm{Pd}[53,102]$ die wasserstoffreiche $\alpha^{\prime}$-Phase. Diese Phasen (beide kfz-Gitter) unterscheiden sich durch einen unterschiedlichen Gitterparameter.

\footnotetext{
${ }^{6}$ Die Randlöslichkeit der $\alpha$-Phase von Wasserstoff in Palladium bei Raumtemperatur liegt im Bereich von $0,006 \mathrm{H} / \mathrm{Pd}[38]$ bis $0,017 \mathrm{H} / \mathrm{Pd}[53]$
} 


\section{Das Vanadium-Wasserstoffsystem}

Vanadium ist ein sehr unedles Metall, welches im Kontakt mit Luft eine Oxidschicht bildet, wodurch es vor weiterer Oxidation geschützt ist. Dennoch eignet sich Vanadium gut zur Untersuchung von Wasserstoffversprödungsphänomenen, da eine im Vergleich zu anderen Metallen große Menge an Wasserstoff bei Raumtemperatur lösbar ist, ohne dass es zur Bildung einer neuen wasserstoffreichen Phase kommt, siehe Abbildung 12.

Die Oxidschicht von Vanadium verhindert, dass Wasserstoff ohne weitere Präparation in der Probe gelöst werden kann. Andererseits verhindert die Oxidschicht auch den Austritt von Wasserstoff aus dem Metall [103]. Dieses Problem kann überwunden werden, indem das Oxid in einer Vakuumsputteranlage abgetragen wird und die Stelle mit Palladium bedeckt wird. Durch die Palladiumschicht kann die Probe beladen werden. Das so präparierte Vanadium eignet sich hervorragend für Wasserstoffversprödungsexperimente, da die Proben auch längere Zeit (einige Stunden) an Luft liegen können, ohne dass von einer merklichen Veränderung des Wasserstoffgehalts ausgegangen werden muss.

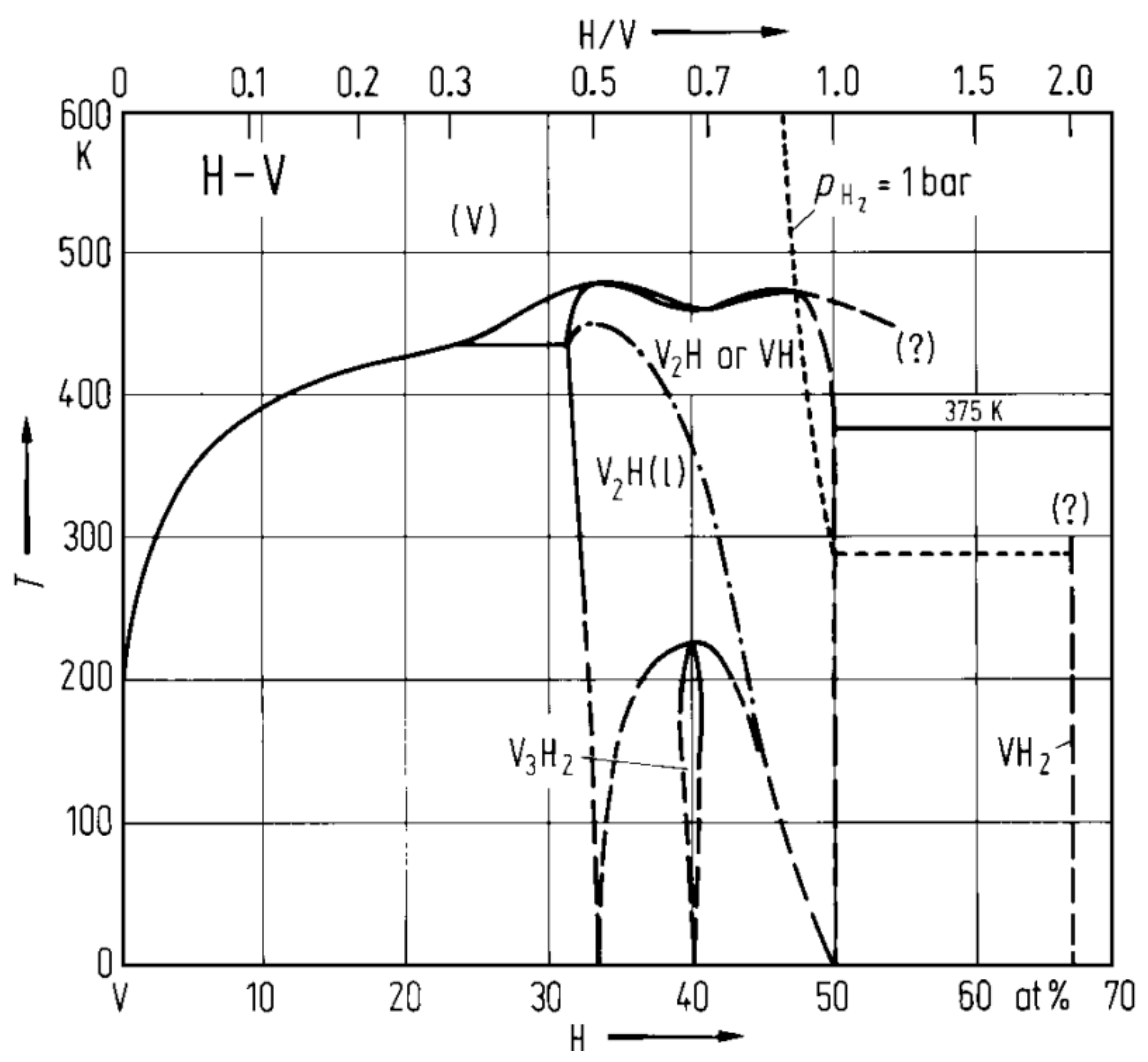

Abb. 13 Phasendiagramm des Vanadium-Wasserstoffsystems [104]. 


\section{Experimentelle Methoden}

Im Rahmen dieser Arbeit wurden verschiedene Aspekte des Einflusses von Wasserstoff auf die Defektbildung und -anordnung untersucht. Um die von Wasserstoff hervorgerufenen Veränderungen beobachten zu können, wurde ein breites Spektrum an Geräten und Apparaten verwendet. Um zielgerichtete Beobachtungen zu ermöglichen, mussten Versuchsapparaturen erweitert oder gänzlich neu entworfen werden.

Im Folgenden werden die verwendeten Geräte vorgestellt. Des Weiteren werden grundlegende experimentelle Verfahren vorgestellt, welche bei verschiedenen Versuchen verwendet wurden.

\subsection{Probenpräparation}

\subsubsection{Elektrolytisches Beladen}

Eine Metallprobe kann elektrolytisch mit Wasserstoff beladen werden, indem die Probe in einem Elektrolyten als negativ polarisierte Kathode geschaltet wird. Als Anode dient aufgrund der Beständigkeit gegen Oxidation eine Platinelektrode.

In den in dieser Arbeit vorgestellten Versuchen wird ein Elektrolyt bestehend aus $1 / 3$ Phosphorsäure und 2/3 Glyzerin verwendet. Dieser wurde vor der Verwendung mindestens 12 Stunden mit Argon durchflutet, damit gelöster Sauerstoff verdrängt wird. Gelöster Sauerstoff im Elektrolyten würde dazu führen, dass Wasserstoff an der Oberfläche zu Wasser reagieren würde und somit die Wasserstoffkonzentration im Metall unbekannt wäre.

Ein vorgegebener Wasserstoffgehalt kann unter Verwendung des Faradayschen Gesetzes eingestellt werden:

$$
\Delta c=\frac{I \cdot t}{F} \cdot \frac{1}{n_{M}}
$$

$\Delta c$ ist die Änderung des Wasserstoffgehalts in Mol Wasserstoff / Mol Palladium, I der während der Beladung (konstant) fließende Strom zwischen Probe und Anode. Somit ergibt sich mit der Zeit $t$ die geflossene Ladung, welche mit der Faraday Konstante $F$ in Stoffmenge umgerechnet wird. Wird die Probe hinreichend langsam beladen ${ }^{7}$ bewirkt jedes geflossene Elektron die Lösung eines Wasserstoffatoms und somit ist die geflossene Ladung ein Maß für den gelösten Wasserstoff. Daraus folgt, dass $\Delta c$ bestimmt werden kann, wenn die Stoffmenge der Probe $n_{M}$ bekannt ist.

Die bisher vorgestellte Methode kann nur verwendet werden, wenn die vom Elektrolyten abgespaltenen Wasserstoffatome schnell genug ins Metall diffundieren. Jedoch haben einige Metalle eine Oxidschicht, die dies verhindern [103]. Um Vanadium zu beladen, wird eine Palladiumschicht aufgetragen, durch die die Beladung ermöglicht wird. In dieser Arbeit wurden daher in einer Vakuumsputteranlage mit Hilfe von Magnus $\mathrm{Hamm}^{8}$ und Ryota Gemma ${ }^{8}$ zunächst die Oxidschicht von einer Seite der Proben entfernt und anschließend eine $20 \mathrm{~nm}$ dicke Palladiumschicht aufgetragen, durch die die Beladung ermöglicht wird.

\section{Entladen einer Probe}

Um den Wasserstoff aus einer Probe zu entfernen, gibt es zwei Methoden: Zum einen kann die Probe elektrolytisch entladen werden, zum anderen kann die Probe an Luft ausgeheizt werden. Die elektrolytische Entladung erfolgt, indem die Probe als Anode geschaltet wird. Dazu wird eine feste Spannung ${ }^{9}$ (für Palladium wurde eine Spannung von $0,78 \mathrm{~V}$ verwendet) angelegt und der

\footnotetext{
${ }^{7} \mathrm{Im}$ Fall von Palladium kann eine Stromdichte von $0.3 \frac{\mathrm{mA}}{\mathrm{cm}^{2}}$ verwendet werden [105].

${ }^{8}$ Institut für Materialphysik, Universität Göttingen

${ }^{9}$ Die Spannung sollte nicht ausreichen, um Wasser zu spalten. Des Weiteren sollte die Spannung zu keiner Oxidation der Probe führen oder die Probe sogar auflösen.
} 
fließende Strom gemessen. Dieser Strom ist ein Maß dafür, wie viel Wasserstoff die Probe verlässt. Versiegt dieser Strom befindet sich kein Wasserstoff mehr in der Probe. Nachteil dieser Methode ist neben unerwünschten chemischen Reaktionen, dass fest gebundener Wasserstoff, bspw. an Versetzungen, evtl. nur teilweise entfernt werden kann.

Die Entladung durch Ausheizen an Luft beruht darauf, dass bei höheren Temperaturen Wasserstoff schneller diffundiert. An der Oberfläche reagiert der Wasserstoff mit Sauerstoff zu Wasser. Diese Methode erlaubt es zuverlässig Wasserstoff zu entfernen, allerdings müssen dazu Temperaturen oberhalb von $200^{\circ} \mathrm{C}$ verwendet werden. Bei diesen Temperaturen kommt es im Falle von verformtem Palladium bereits zu ersten Erholungserscheinungen [48].

\subsubsection{Präparation von Metalloberflächen zur Untersuchung durch Nanoindentation}

Um wiederholbare Nanoindentationsversuche durchzuführen, wird eine Probenoberfläche benötigt, welche eine mittlere Oberflächenrauigkeit von wenigen Nanometern aufweist. Zudem sollte die Versetzungsdichte so gering wie möglich sein, damit die Wahrscheinlichkeit groß ist mit dem Nanoindenter eine versetzungsfreie Region zu treffen. Des Weiteren muss die Probe so präpariert werden, dass Wasserstoff in ihr gelöst werden kann.

In dieser Arbeit wurden Einkristalle aus Vanadium und polykristallines Palladium verwendet. Diese Proben wurden zunächst mit SiC-Schleifpapier in Wasser bis zu einer Körnung von 2400 geschliffen. Die weiteren Schritte unterscheiden sich für die einzelnen Metalle.

\section{Vanadium}

Bei den verwendeten Vanadiumproben ${ }^{10}$ handelt es sich um (001)-orientierte einkristalline Scheiben mit einem Durchmesser von $12 \mathrm{~mm}$ und einer Dicke von $2 \mathrm{~mm}$.

Die Einkristalle wurden nach dem Schleifen unter Verwendung von feiner werdenden Diamantpolierpasten bis zu einer Partikelgröße von $0,04 \mu \mathrm{m}$ poliert. Anschließend wurden die Einkristalle in einer Lösung aus Methanol und Schwefelsäure (5:1 Volumenanteile) bei 6,5 V für ca. 2 Minuten elektropoliert. Danach wurden die Einkristalle in Tantalfolie verpackt und bei $800^{\circ} \mathrm{C}$ für 24 Stunden im Vakuum bei $p \approx 10^{-7}$ mbar ausgelagert, um Defekte auszuheilen. Die Tantalfolie dient dazu den Sauerstoffpartialdruck um die Probe zu reduzieren. Damit keine neuen Defekte entstehen, wurden die Proben im Ofen langsam abgekühlt.

Um die Vanadiumproben mit Wasserstoff beladen zu können, wird von einer Seite in einer Vakuumsputteranlage die Oxidschicht abgetragen und eine Palladiumschicht aufgebracht.

Die so erhaltenen Proben können nun für verschiedene Versuche verwendet werden, da gelöster Wasserstoff durch Ausheizen bei $200^{\circ} \mathrm{C}$ an Luft wieder entfernt werden kann.

\section{Palladium}

Bei den verwendeten Palladiumproben ${ }^{11}$ handelt es sich um polykristallines Blech mit einer Dicke von $500 \mu \mathrm{m}$.

Die Präparation von glatten Palladiumoberflächen ist aufwendig. Dabei ist auf eine saubere Arbeitsweise zu achten, da kleinste Verunreinigungen zum Verkratzen der Oberfläche führen. Nach dem Schleifen mit SiC-Papier mit einer Körnung von 2400, wird die Palladiumprobe zunächst mit einer Diamantpaste mit $3 \mu \mathrm{m}$ Körnung bei hoher Umdrehungszahl und mit hohem Anpressdruck auf einer langflorigen Polierscheibe (MD-Plus von Struers) für 5-10 Minuten poliert. Anschließend wird die Geschwindigkeit herabgesetzt und für 5-10 Minuten unter Verwendung eines geringen Anpressdrucks poliert. Als letzter mechanischer Polierschritt wird die Probe auf einem feinem Poliertuch (MD-Chem von Struers) unter Verwendung von Wasser per Hand mit wenig Druck für 2-5 Minuten poliert.

\footnotetext{
${ }^{10}$ Erworben bei Goodfellow mit einer Reinheit von 99,99\%

${ }^{11}$ Erworben bei Wieland Edelmetalle GmbH mit einer Reinheit von 99,95\%
} 
Der letzte Polierschritt ist das sog. Elektrowischpolieren, siehe Abbildung 14. Hierbei wird die Probe an eine senkrecht stehende Polierscheibe, welche in einen Elektrolyten bestehend aus $149 \mathrm{~mL}$ $\mathrm{H}_{2} \mathrm{O}, 9,1 \mathrm{~g} \mathrm{Na}_{2} \mathrm{~S}_{2} \mathrm{O}_{3}, 12,7 \mathrm{~g} \mathrm{KSCN}$ und $10,9 \mathrm{~g} \mathrm{NH} \mathrm{Nl}_{4} \mathrm{Cl}$ (nach [106]) eingetaucht ist, gefahren. Die Probe ist durch den Elektrolyten benetzt, jedoch berührt die Probe nicht die Polierscheibe. Das Polieren wird für 30 Sekunden bei einer Stromdichte von $1,5 \mathrm{~A} / \mathrm{cm}^{2}$ durchgeführt.

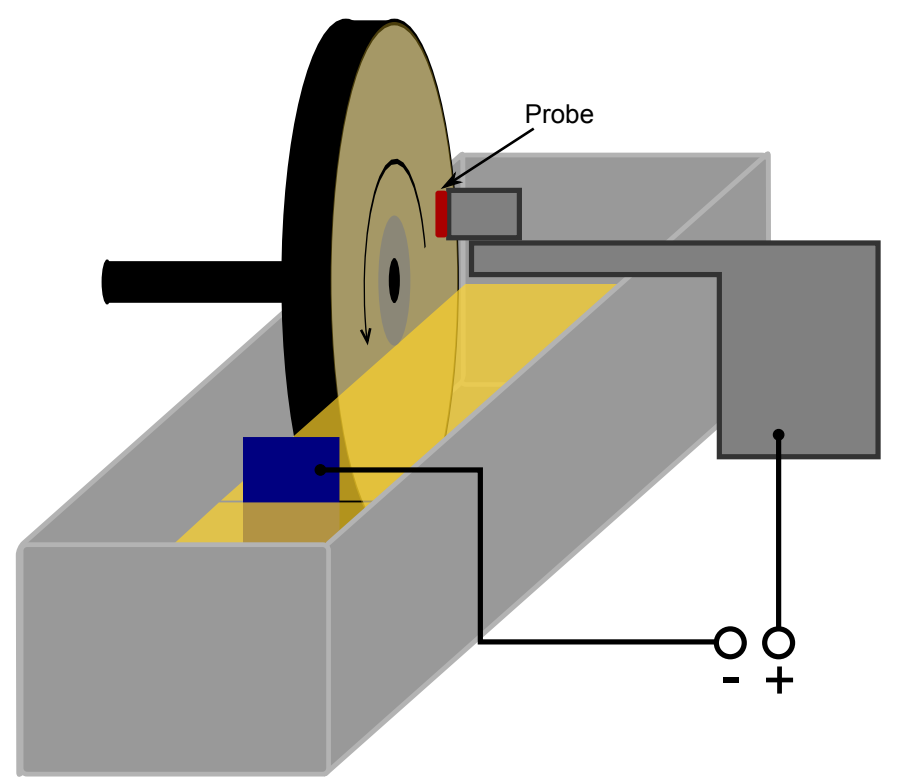

Abb. 14 Schematische Darstellung des Elektrowischpolierens. Eine Polierscheibe mit einem feinen Poliertuch wird senkrecht in einem Elektrolyten gedreht. Die Probe wird oberhalb der Flüssigkeit an die feuchte Polierscheibe herangefahren. Zwischen Probe und Polierscheibe kann eine Spannung angelegt werden.

Um eine geringe Versetzungsdichte zu erhalten, werden die Proben bei $900^{\circ} \mathrm{C}$ im Vakuum für 48 Stunden bei $p \approx 10^{-7}$ mbar ausgelagert. Durch das Auslagern kommen die einzelnen Körner zum Vorschein, indem die Korngrenzen einsinken. Das Innere der Körner ist für die Nanoindentation geeignet. Dies erlaubt es Untersuchungen mit verschiedenen Wasserstoffkonzentrationen im gleichen Korn durchzuführen. Ist eine auch makroskopisch glatte Oberfläche erwünscht, kann dies durch erneutes Elektropolieren erreicht werden.

Wasserstoff kann aus der Probe entfernt werden, indem die Palladiumprobe im Elektrolyten als Anode geschaltet wird und eine Spannung von 0,78 V angelegt wird. Im Stromkreis wird der Strom gemessen. Versiegt dieser, ist die Probe wasserstofffrei. 


\subsubsection{Präparation von Vanadiumsäulen im Mikrometerbereich}

Für die Präparation wird ein sog. Focused Ion Beam (FIB) verwendet. Das FIB ist ein Rasterelektronenmikroskop (s.u.), welches neben einem Elektronenstrahl über einen Galliumionenstrahl verfügt, mit dem Material aus einer Oberfläche gezielt entfernt werden kann.

Bevor in das Vanadium mit einem FIB die Säulen hineingeschnitten werden, wird die Vanadiumprobe mit Wasserstoff beladen. Die Präparation von Mikrosäulen wird normalerweise in mehreren Schritten durchgeführt. Als erstes wird ein breiter Graben ausgehoben (Durchmesser ca. $30 \mu \mathrm{m}$ ). Dieser Graben hat zum einen die Aufgabe Platz für die Indenterspitze zu schaffen und zum anderen soll ein großes freies Volumen um die eigentliche Säule geschaffen werden, damit Material, welches beim Feinschnitt abgetragen wird sich nicht direkt an der Säule wieder absetzen kann. Danach wird die Säule in mehreren Schritten weiter auf den Enddurchmesser heruntergeschnitten. Um Strahlenschäden zu vermeiden wird mit dem Strahl von außen nach innen geschnitten.

Dabei ergibt sich keine ideale Säule, sondern eine nach oben hin schmaler werdende Säule, siehe dazu Abbildung 15.
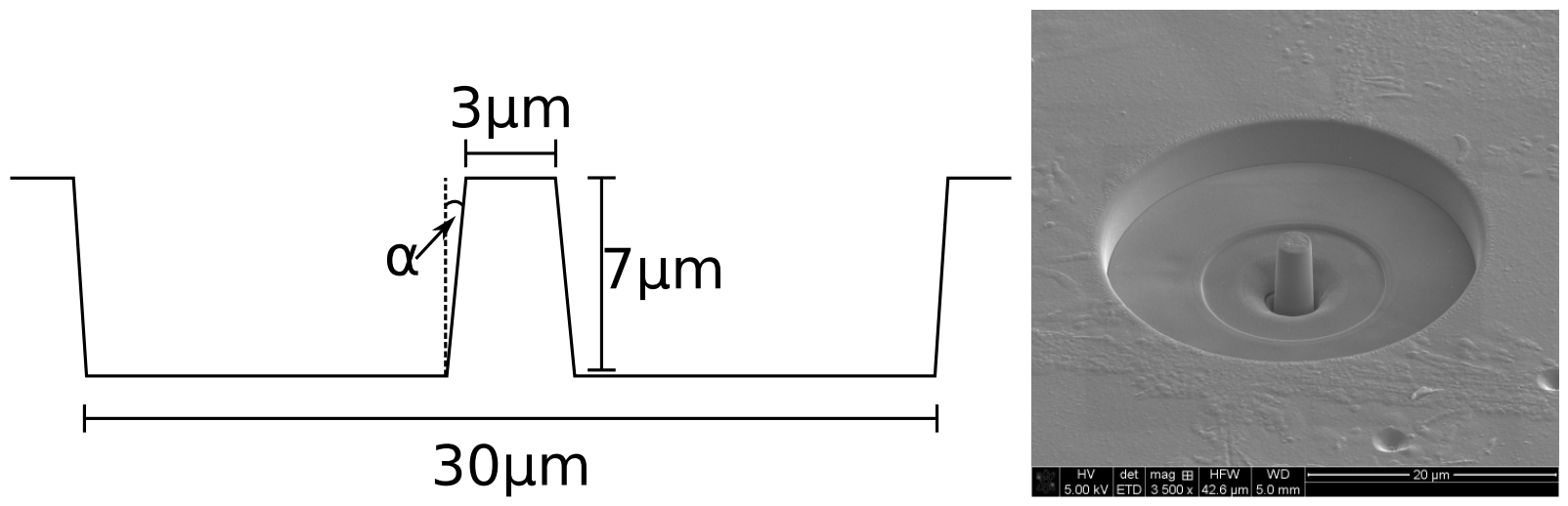

Abb. 15 Veranschaulichung der verwendeten Geometrie der Säulendruckversuche. Links: Schematische Querschittsdarstellung einer Säule; Rechts: REM Aufnahme einer fertig präparierten Vanadiumsäule.

\subsubsection{Präparation von TEM-Proben aus Palladiumblech}

Eine geeignete Probe für die Transmissionsenelektronenmikroskopie (TEM) sollte wenige $10 \mathrm{~nm}$ dünn sein. Ferner sollten durch die Präparation keine weiteren Defekte in der Probe erzeugt werden.

Eine Methode dies zu erreichen, ist die Präparation mittels elektrolytischem Ätzen.

Zuerst wird eine Scheibe (Durchmesser $3 \mathrm{~mm}$ ) aus dem Probenmaterial ausgestanzt und auf ca. $100 \mu \mathrm{m}$ geschliffen. Danach werden runde Vertiefungen in beide Seiten der Probe geschliffen. Üblicherweise wird die Probe so geschliffen, dass diese an der dünnsten Stelle eine Dicke von weniger als $30 \mu \mathrm{m}$ aufweist.

Anschließend wird die Probe in einem Tenupol der Firma Struers elektrolytisch weiter gedünnt, bis in der Mitte ein Loch entsteht. Dadurch wird die Probe an den Rändern des Loches im Allgemeinen hinreichend dünn.

Für die TEM-Proben Präparation von Palladium wird ein (Ätz-)Elektrolyt aus $70 \%$ Essigsäure $(96 \%)$ und $30 \%$ Perchlorsäure $(60 \%)$ verwendet [107]. Der Ätzvorgang findet bei ca. $10^{\circ} \mathrm{C}$ statt. Dabei werden eine Platin-Kathode und eine Spannung von $30 \mathrm{~V}$ verwendet. Durch diese Einstellung fließt ein Strom von ca. 0.25 A und der Ätzvorgang benötigt je nach Dicke der Probe wenige Sekunden bis maximal 5 Minuten. 


\subsection{Verformungsmethoden}

\subsubsection{Nanoindentation}

Bei der Nanoindentation wird ein sog. Indenter in die Oberfläche eines Materials getrieben. Für die Versuche in dieser Arbeit wurde ein Nanoindenter von Typ NANO G200 der Firma Nano Instruments verwendet. Bei der Nanoindentation wird die Eindringtiefe als Funktion der auferlegten Kraft gemessen. Die Spitze des Indenters kann verschiedene Formen haben, in dieser Arbeit wurde eine Berkovichgeometrie verwendet. Hierbei handelt es sich um einen flache dreiseitige Pyramide mit einem Gesamtwinkel von $142,3^{\circ}$. Diese Spitzen sind typischerweise am Ende abgerundet und weisen einen Spitzenradius im Bereich von $100 \mathrm{~nm}$ auf.

Aufgrund dieser Geometrie wird nur ein kleines Volumen verformt, wodurch zum einen viele Versuche an einer Probe möglich sind und zum anderen die Wahrscheinlichkeit, ein defektfreies Gebiet zu treffen, hoch ist $[108,109]$.

In-situ Nanoindentation Für die Untersuchungen an Palladium wird ein in-situ Aufbau (Abbildung 16) benötigt, da Wasserstoff ansonsten während des Versuchs die Probe verlassen würde. Die Untersuchungen an Palladium werden daher in einer in-situ Zelle [110], welche von Eike Epler ${ }^{12}$ entwickelt wurde und in Rahmen dieser Arbeit an die Untersuchungen von Palladium angepasst wurde, durchgeführt. In dieser Zelle befindet sich die Probe im Elektrolyten. Dies ermöglicht zum einen die elektromotorische Kraft (EMK) mittels einer Silber/Silberchlorid Bezugselektrode zu bestimmen. Die EMK hängt von der Wasserstoffkonzentration ab. Wird also die EMK mit einem Potentiostaten konstant gehalten, wird genau soviel neuer Wasserstoff in der Probe gelöst, wie hinaus diffundiert.

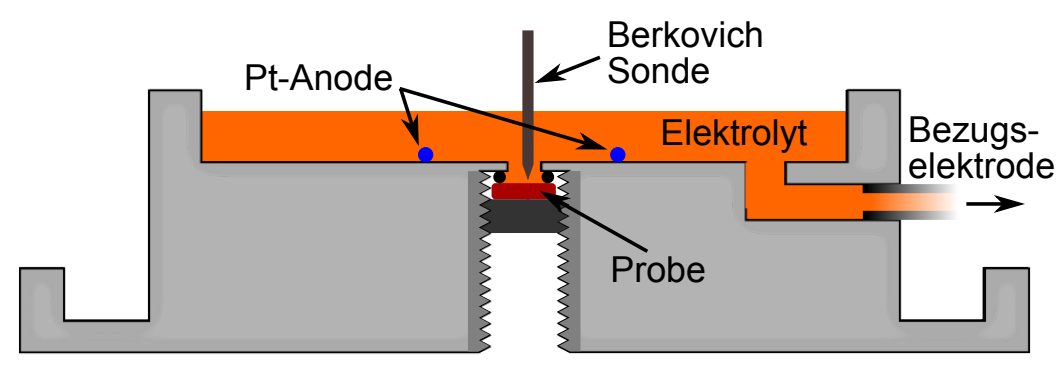

Abb. 16 Schematische Darstellung eines in-situ-Aufbaus zur Nanoindentation im Elektrolyten. Mit Hilfe einer Bezugselektrode kann die elektromotorische Kraft (EMK) bestimmt werden. Diese ist anhängig von der Wasserstoffkonzentration. Durch einen Potentiostaten kann die EMK konstant gehalten werden, $d$. h. verloren gegangener Wasserstoff wird während des Versuchs durch neuen Wasserstoff ersetzt.

\footnotetext{
${ }^{12}$ Institut für Materialphysik, Universität Göttingen
} 


\subsubsection{Kaltwalzen von Palladiumblechen}

Um den Einfluss von Wasserstoff auf die Bildung von Versetzungsnetzwerken bei Verformung zu studieren, wurde Palladium zunächst bei $900^{\circ} \mathrm{C}$ für 48 Stunden im Vakuum bei $p \approx 10^{-7}$ mbar ausgelagert, um vorhandene Versetzungen auszuheilen und um eine definierte Ausgangssituation sicherzustellen.

Anschließend wurde Wasserstoff elektrolytisch im Palladium gelöst. Danach wird die PalladiumWasserstofflegierung sofort mit einer Walze verformt, wobei auf jede Probe die gleiche Verformung (Reduzierung der Dicke auf $50 \%$ ) angewendet wird.

Der nach der Verformung noch vorhandene Wasserstoff wurde elektrolytisch entfernt. Der Versuchsablauf ist in Abbildung 17 schematisch gezeigt.

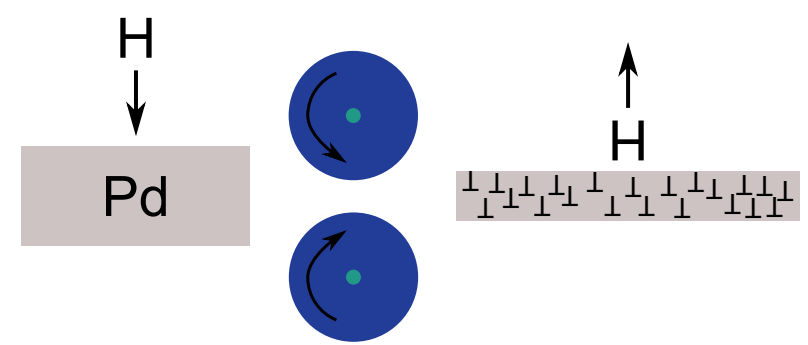

Abb. 17 Schematische Darstellung des Kaltwalzen von Palladium-Wasserstofflegierungen 


\subsubsection{Hochdruck-Torsion}

Wird eine Probe unter hohem Druck einer Torsionsspannung ausgesetzt, können sehr große Dehnungen erreicht werden [111]. Hierzu wird eine Metallprobe mit einem Druck im GPa-Bereich in einer hydraulischen Presse belastet und gleichzeitig werden die Stempel der Presse gegeneinander verdreht. Dabei wird im Regelfall eine Rotationsgeschwindigkeit unterhalb von $1 \mathrm{~min}^{-1}$ verwendet.

Diese HPT genannte Methode (engl. High-Pressure Torsion) erlaubt es, neben diversen anderen Möglichkeiten [112], Proben höchsten Verformungen auszusetzen, wodurch Korngrößen mit Durchmessern von wenigen $100 \mathrm{~nm}[113,114]$ oder sogar unter $100 \mathrm{~nm}$ [115-118] erreicht werden können.

HPT findet auch Verwendung in zweistufigen Anwendungen. So kann HPT verwendet werden, um Pulverproben zu kompaktieren [119] oder um den Effekt von Verformung auf bereits nanokristalline Proben zu untersuchen [120, 121].

In Abbildung 18 ist eine schematische Darstellung des verwendeten Aufbaus zu sehen, welcher an der Universität Wien und in Zusammenarbeit mit der Arbeitsgruppe von Prof. Dr. M. Zehetbauer ${ }^{13}$ benutzt wurde. Dieser Aufbau ermöglicht es, Proben mit einem Durchmesser von $8 \mathrm{~mm}$ zu verformen, des Weiteren kann der Aufbau mit Trockeneis gekühlt werden $\left(\mathrm{T}_{\mathrm{CO}_{2}} \approx-60^{\circ} \mathrm{C}\right)$.

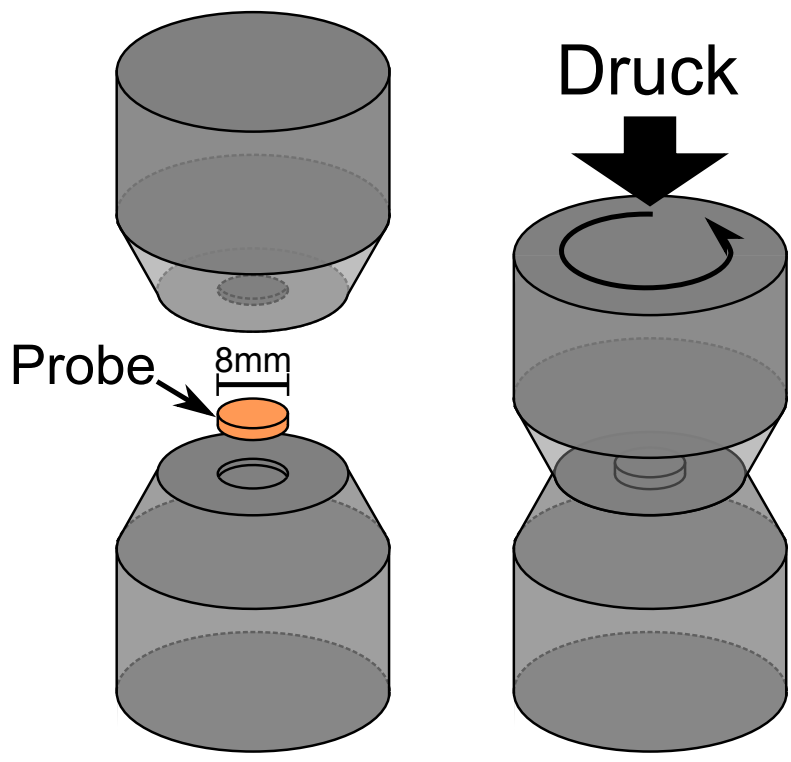

Abb. 18 Schematischer Aufbau einer High-Pressure Torsion Verformungsanlage. Ein solcher Aufbau ermöglicht es Proben mit einem Durchmesser von $8 \mathrm{~mm}$ unter Druckbelastung $\mathrm{zu}$ verdrillen.

\footnotetext{
${ }^{13}$ Groups Physics of Nanostructured/Functional Materials, Universität Wien
} 


\subsection{Probencharakterisierung}

\section{Transmissionselektronenmikroskopie}

Mit dem Transmissionselektronenmikroskop (TEM) können kleinste Strukturen darstellt werden [124]. Dazu werden Proben benötigt, die mit Elektronen durchstrahlt werden können und somit nur wenige $10 \mathrm{~nm}$ dick sein dürfen. Im TEM wird ein Elektronenstrahl auf die Probe fokussiert und somit ein Durchstrahlbild erzeugt. Für materialwissenschaftliche Anwendungen werden Beschleunigungsspannungen im Bereich von $120 \mathrm{kV}$ bis $300 \mathrm{kV}$ und mehr verwendet.

Die Kontrasterzeugung erfolgt auf verschiedene Art und Weise. Die wichtigsten Mechanismen sind Streuung von Elektronen, dies hängt von der Probendicke aber auch von der Ordnungszahl der Atome ab und Beugung der Elektronen an kristallinen Ebenen.

Um die Beugung der Elektronen auszunutzen, um einen Kontrast zu erzeugen, wird das Beugungsbild verwendet. Durch eine Blende können z. B. die ungebeugten Elektronen ausgewählt werden. Wird in das Realraumbild zurückgeschaltet so ergibt sich ein sog. Hellfeldbild. Werden hingegen gebeugte Elektronen ausgewählt ergibt sich ein Dunkelfeldbild.

Des Weiteren kann auch das Beugungsbild aufgenommen werden. Bei kristallinen Proben ergibt sich ein Punktemuster, aus dem auf die Kristallstruktur und die Orientierung der Probe geschlossen werden kann. Sind mehrere Körner im Strahlengang kommt es zu einer Überlagerung der einzelnen Beugungsbilder. Dies kann beim Ausleuchten vieler Körner dazu führen, dass im Beugungsbild Ringe sichtbar werden.

\section{Environmental TEM}

Ein Environmental TEM (ETEM) (vgl. Abbildung 19) bietet die Möglichkeit, TEM Untersuchungen in Gasatmosphäre durchzuführen. Dies ist nur möglich, wenn besondere Vorkehrungen unternommen werden, um die Probe einer Atmosphäre auszusetzen und gleichzeitig den Rest des Mikroskops in Ultrahochvakuum zu halten. Um dies zu erreichen, gibt es prinzipiell zwei Möglichkeiten: Zum einen kann ein spezieller Probenhalter verwendet werden, welcher eine elektronendurchsichtige Kammer besitzt, in der sich Probe und Gas befinden. Diese Möglichkeit erlaubt es Gasdrücke bis zu 400 mbar zu verwenden [125], jedoch ist die Auswahl der Haltertypen begrenzt und es können nicht höchste Aufösungen erreicht werden. Die andere Möglichkeit ergibt sich, indem an der Position der Probe Gas in den Strahlengang des TEM eingelassen wird, welches wiederum mit Hilfe von mehreren Turbomolekularpumpen oberhalb und unterhalb der Probe abgepumpt wird, sodass im restlichen Teil des Strahlengangs Hochvakuumbedingungen vorherrschen [122]. 
Diese Methode erlaubt es am FEI Titan environmental TEM in Göttingen Gasdrücke je nach Gassorte von bis zu 20 mbar (z. B. Argon, Stickstoff) [123] einzustellen. Aufgrund der besonders

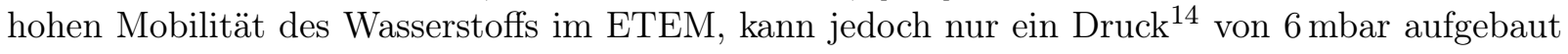
werden.

Des Weiteren ermöglicht das verwendete ETEM höchste Auflösungen unter Verwendung von Korrektoren der sphärische Aberration und der chromatische Aberration [123].

\section{Rasterelektronenmikroskopie}

Eine andere Art der Elektronenmikroskopie stellt das Rasterelektronenmikroskop (REM) dar. Bei dieser Methode wird ein Elektronenstrahl, welcher für materialphysikalische Anwendungen typischerweise mit 5 bis $20 \mathrm{kV}$ beschleunigt wird, auf der Probenoberfläche fokussiert. Zusätzlich dazu wird der Strahl über die Oberfläche gerastert. Trifft der Elektronenstrahl auf die Oberfläche, werden verschiedene Vorgänge ausgelöst, mit denen die Oberfläche charakterisiert werden können. Für die topographische Darstellung können z. B. die sog. Sekundärelektronen verwendet werden. Diese entstehen, indem der Primärstrahl Elektronen aus dem Material herausschlägt. Die Sekundärelektronen haben somit eine geringere Energie. Daher können nur Sekundärelektronen den Detektor erreichen, die aus einer Tiefe von 1-10 nm stammen.

Eine anderer Effekt ist die Erzeugung von charakteristischer Röntgenstrahlung. Diese Strahlung kann verwendet werden, um die Probenoberfläche chemisch zu analysieren.

\section{Rasterkraftmikroskopie}

Für die topologische Darstellung einer Oberfläche kann das Rasterkraftmikroskop (AFM, engl. Atomic Force Microscope) verwendet werden. Bei diesem Instrument rastert eine Spitze die Oberfläche ab. Die Spitze wird hierzu mit ihrer Resonanzfrequenz in Schwingung versetzt. Kommt die schwingende Spitze der Oberfläche nahe, wird eine Kraft auf die Spitze ausgeübt, ohne dass die Spitze die Oberfläche berührt. Dadurch wird die Schwingfrequenz verändert. Die Änderung der Frequenz ist ein Maß für die Höhenänderungen der Probenoberfläche. Die so erhaltenen Aufnahmen haben eine besonders hohe Auflösung normal zu Probenoberfläche. Somit können bei geeigneten Proben atomare Stufen auf der Oberfläche sichtbar gemacht werden.

\footnotetext{
${ }^{14}$ Messungen von Andreas Kelling, Materialphysik Universität Göttingen
} 


\subsection{Molekulardynamiksimulationen}

Molekulardynamiksimulationen (MD) erlauben es ein besseres Verständnis der Vorgänge im Inneren von Metallen bei der Verformung zu erhalten. Durch die Simulation jedes einzelnen Atoms in einem virtuellen Versuch kann die Entstehung und Entwicklung von Kristallbaufehlern studiert werden. Darüber hinaus können weitere physikalische Größen bestimmt werden, dies ist in dieser Arbeit bspw. die Kraft, die der Nanoindenter benötigt, um die simulierte Metallprobe zu verformen.

Ziel der Untersuchungen in dieser Arbeit ist es, zum einen die generelle Versetzungsentstehung zu untersuchen und zum anderen den Einfluss von Wasserstoff auf die Versetzungsbildung und Entwicklung bei Nanoindentationsversuchen zu zeigen. Die in dieser Arbeit verwendete Herangehensweise beruht auf der Embedded Atom Method (EAM), welche von Daw und Baskes [126-128] entwickelt wurde. Der Einsatz dieser Methode zur Ergründung der Wasserstoffversprödung geht auf Baskes, et al. zurück [129, 130], hier wurde das Nickel-Wasserstoffsystem untersucht. Dieses System ist daher auch das am besten untersuchte System [96, 129-135]. Weitere MetallWasserstoffsysteme, die mit der EAM-Methode untersucht wurden sind: Fe-H [136-138], Pd-H [139] und V-H $[98,140]$. MD-Simulationen beruhen darauf, dass empirische Potentiale, welche die Interaktion von Atomen untereinander beschreiben, an die jeweiligen Atomsorten angepasst werden. Für die Untersuchungen wurden aus der Literatur bekannte Potentiale und Ansätze zur Simulation von Nanoindentationsversuchen verwendet, um ein kfz Material und ein krz Material zu untersuchen.

Für diese Methode werden neben den atomaren Potentialen $\phi_{M M}\left(r_{i j}\right), \phi_{M H}\left(r_{i j}\right)$ und $\phi_{H H}\left(r_{i j}\right)$ (Metall-Metall, Metall-Wasserstoff, Wasserstoff-Wasserstoff im Abstand $r_{i j}$ ) auch zwei Elektronendichtefunktion $\rho_{M}(r)$ und $\rho_{H}(r)$ benötigt, welche die Elektronendichte im Abstand $r$ vom (Metall-, bzw. Wasserstoff-) Atom angeben. Des Weiteren wird je eine Funktion $F_{M}(\rho)$ bzw. $F_{H}(\rho)$ benötigt, die die Reaktion eines Atoms auf die Elektronendichte beschreibt. Die potentielle Energie eines Atom $i$ ergibt sich zu [127, 128]:

$$
E=F_{i}\left(\sum_{j \neq i} \rho_{j}\left(r_{i j}\right)\right)+\sum_{j \neq i} \phi_{i j}\left(r_{i j}\right)
$$

wobei die Funktionen $F_{i}, \rho_{i}$ und $\phi_{i j}$ je nach beteiligten Atomen $i$ und $j$ gewählt werden.

Für die Simulation wurde die Orts- und Geschwindigkeitsaktualisierung durch den Velocity-VerletAlgorithmus [141] vorgenommen und als Thermostat ${ }^{15}$ wurde ein Nosé-Hoover Thermostat $[142,143]$ verwendet, um numerische Ungenauigkeiten auszugleichen.

Gleichung 3.2 zeigt, dass es sich um ein $N^{2}$-Problem ${ }^{16}$ handelt. Um Rechenzeit zu sparen wurde im Rahmen dieser Arbeit ein Algorithmus entwickelt, um dies auf ein $N$-Problem zu reduzieren. Es wird angenommen, dass sich die nächsten Nachbarn eines Atoms nur selten ändern und dass die Potentiale $\phi_{i j}$ bzw. die Elektronendichte $\rho_{i}$ nur eine begrenze Reichweite haben. Daher wird für jedes Atom eine Liste der möglichen Interaktionspartner angelegt, welche in regelmäßigen Abständen aktualisiert wird. Um auch dieses Aktualisierungsproblem auf ein $N$-Problem zu reduzieren, wird ein dreidimensionales Netz von virtuellen Punkten, welches einem einfach kubischen Gitter mit einem Gitterparameter, der der Hälfte des Metallgitterparameter entspricht, angelegt. Auf jeden Punkt $P$ im Netz kann entsprechend seiner Position zugegriffen werden ${ }^{17}$. Im ersten Schritt wird jedem Netzpunkt im Umkreis ${ }^{18}$ eines Atoms dessen Index zugeordnet, in einem zweiten Schritt ergeben die Einträge des zum Atom nächsten Netzpunkt die Liste der nächsten Nachbarn.

\footnotetext{
${ }^{15}$ Ein Thermostat ist in diesen Kontext eine Verfahrensweise, um den simulierten Atomen eine vorgegebene Temperatur zu geben und diese zu halten.

${ }^{16} N^{2}$-Problem ist eine Berechnung, deren Zeitaufwand quadratisch mit der Problemgröße (hier der Atomanzahl) wächst.

${ }^{17}$ Umsetzung im Programmcode: Das Punktenetz ist ein dreidimensionaler Array, auf dessen Einträge wie folgt zugegriffen wird: $P(\vec{x})=P\left[\operatorname{runde}\left(\frac{\vec{x} \cdot x}{a / 2}\right)\right.$, runde $\left(\frac{\vec{x} \cdot y}{a / 2}\right)$, runde $\left.\left(\frac{\vec{x} \cdot z}{a / 2}\right)\right], \vec{x}$ ist eine Raumkoordinate und $a$ der Gitterparameter des Metallgitters.

${ }^{18}$ Der Radius ergibt sich aus der Reichweite der Potentiale.
} 


\section{Versuchsübersicht}

Ziel dieser Arbeit war es den Einfluss von Wasserstoff auf Kristallbaufehler in Metallen darzustellen. Dazu wurden die Versuche hauptsächlich an den Modellsystemen Palladium-Wasserstoff und Vanadium-Wasserstoff durchgeführt.

Die in dieser Arbeit durchgeführten Versuche dienen dazu, die Auswirkungen von Wasserstoff, angefangen von Effekten auf der Atomskala bis hin zu Effekten im Mikrometerbereich, zu untersuchen.

Im Folgenden werden die einzelnen Versuche kurz vorgestellt. Dabei wird neben der Beschreibung der Versuchsdurchführung auch auf das Ziel der Untersuchungen eingegangen.

\subsection{Untersuchung des Einflusses von Wasserstoff auf die Versetzungsnuklea- tion bei Nanoindentationsversuchen}

Wird ein einkristallines Material mit einer spitzen Sonde (Spitzenradius typischerweise $\leq 100 \mathrm{~nm}$ ) indentiert, ist die Wahrscheinlichkeit hoch, einen Bereich zu treffen, der keine Defekte aufweist. Das verformte Material reagiert zunächst elastisch, d. h. das Material kehrt bei Entlastung in seinen Ausgangszustand zurück. Wird das Material darüber hinaus belastet, ordnen sich vorhandene Defekte irreversibel neu an oder neue Defekte entstehen. Es kommt zu einer plastischen Verformung [45]. Wird ein defektfreies Material verformt müssen folglich im plastischen Bereich neue Defekte entstehen [144].

Die Untersuchung der Entstehung neuer Defekte in ehemals defektfreiem Material erlaubt es, Aussagen über die Defektbildungsenergie und, wenn Wasserstoff im Material gelöst ist, den Einfluss von Wasserstoff darauf darzustellen.

Aufgrund der Geometrie des Nanoindenters wird nur ein kleines Volumen verformt, wodurch zum einen viele Versuche an einer Probe möglich sind und zum anderen die Wahrscheinlichkeit, ein defektfreies Gebiet zu treffen, hoch ist [108, 109]. Dadurch erlaubt die Nanoindentation es, den Übergang vom elastischen ins plastische Verhalten in einkristallinen Proben zu beobachten [145147] (siehe Abbildung 20).

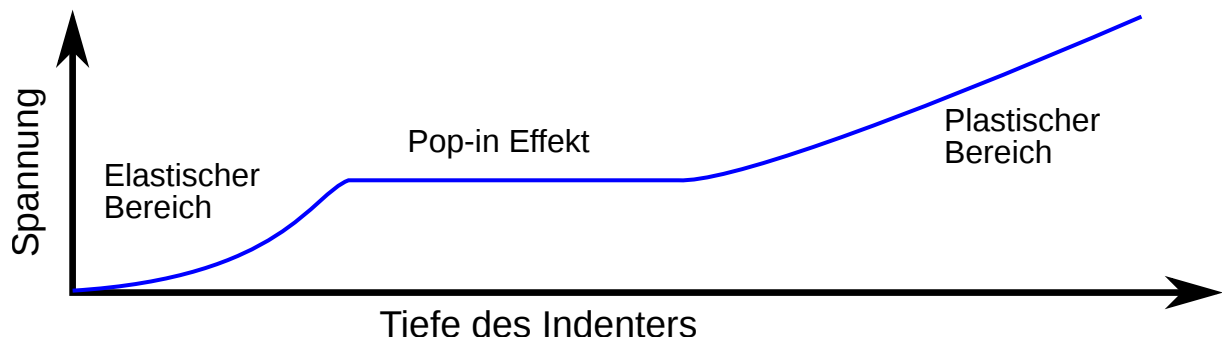

Abb. 20 Theoretische Verformungskurve einer einkristallinen Probe bei

Nanoindentationsversuchen. Bei kleinen Eindringtiefen des Indenters reagiert das Material elastisch. Die Kurve kann mit dem Hertzschen Kontakt Modell [145, 148] beschrieben werden.

Ab einer bestimmten Belastung reagiert die Probe mit plastischem Verhalten. Bei diesem Übergang werden erste Versetzungen gebildet, was zum Abbau von Spannung führt. Dies ist sichtbar anhand des „Pop-in“ Effekts.

In typischen Kraft-Dehnungs-Kurven (siehe Abbildung 20) verhält sich das Material zunächst elastisch. Die Abhängigkeit der Kraft von der Eindringtiefe kann mit dem Hertzschen Kontakt Modell $[145,148]$ beschrieben werden. Die aufzuwendende Kraft $F$ lässt sich durch eine Funktion der Form $F=a \cdot d^{b}$ beschreiben. Hierbei ist $d$ die Eindringtiefe und $a$ und $b$ sind freie Parameter. Der Übergang vom elastischen in den plastischen Bereich wird durch einen waagerechten Abschnitt (Pop-in Effekt) sichtbar [109, 144-147]. Durch das Bilden der ersten Defekte wird Spannung abgebaut und die Sonde gleitet in kurzer Zeit weit ins Material. 
Beruht der Pop-in Effekt auf der Bildung von Versetzungen, ist die Kraft, bei der der Pop-in Effekt auftritt, abhängig von der Bildungsenergie der Versetzungen. Daher ermöglicht diese Methode den Einfluss von gelösten Fremdatomen auf die Bildungsenergie von Versetzungen, wie es im Defactant-Konzept (Kapitel 2.3) beschrieben wird, direkt zu beobachten.

Weitere Informationen über das Verhalten von Versetzungen gibt der zurückbleibende Eindruck in der Oberfläche (vgl. Abbildung 21). Können Versetzungen sich ungestört bewegen, wird die Verformung weit ins Material getragen, die umliegende Oberfläche sinkt ein, die Oberfläche zeigt einen sog. "Sink-In“. Ist dies nicht gegeben, bleibt die Verformung lokal und das verdrängte Material wölbt sich an der Sonde entlang hinauf, dieser Effekt wird „Pile-Up“ genannt [146, 149].
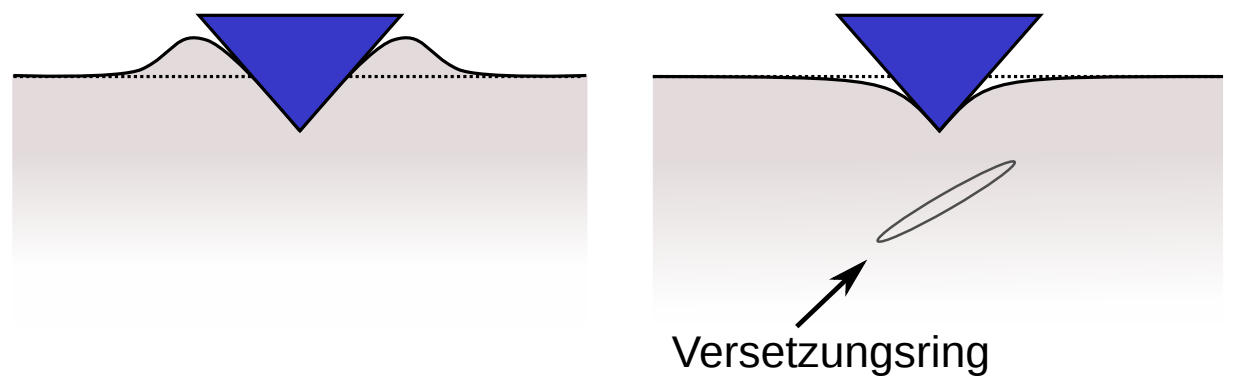

Abb. 21 Schematische Darstellung der Oberfläche nach der Nanoindentation [149]. Wölbt sich das verdrängte Material an der Sonde auf, bedeutet dies, dass die Verformung lokal erfolgt, es kommt zum „Pile-Up“. Ist die Oberfläche nach dem Indentationsexperiment eingesunken, bedeutet dies, dass Versetzungen sich weit bewegen können und somit die Verformung im Material verteilt wird. Dieses Verhalten wird „Sink-In“ bezeichnet.

Im Rahmen dieser Arbeit wurde der Einfluss von Wasserstoff auf den Pop-in Effekt in einem Vanadiumeinkristall und in einer polykristallinen Palladiumprobe untersucht.

Ziel der Untersuchungen war es, die Veränderungen der Defektbildungsenergie darzustellen und die im weiteren Verlauf entstehende Versetzungsinteraktion zu untersuchen, indem die Veränderungen des zurückbleibenden Eindrucks untersucht werden.

Die Untersuchungen an Vanadium wurden mit Inga Knorr ${ }^{19}$ durchgeführt.

Für die Untersuchungen an Palladium wurde ein in-situ Aufbau in Zusammenarbeit mit mit Eike Epler ${ }^{19}$ genutzt.

Zunächst wurden die Probenoberflächen, wie in Kapitel 3.1.2 beschrieben präpariert. Im Falle von Palladium ergeben sich Körner mit Durchmessern im Bereich von 50-400 $\mu \mathrm{m}$. Um eine Vergleichbarkeit der Ergebnisse der Untersuchungen an Palladium zu gewährleisten, wurde ein großes Korn ausgewählt, in dem alle Versuche durchgeführt wurden.

Vor der Untersuchung wurden die Proben zunächst von evtl. vorhandenem Wasserstoff befreit, vgl. Kapitel 3.1.1. Daran anschließend wurden die Proben mit einer vorher festgelegten Wasserstoffkonzentration beladen. Bei Palladium geschah das bereits im in-situ Aufbau. Bei Vanadium wurde ein Kabel an die Probe geklebt, durch welches der elektrische Kontakt hergestellt wurde. Vanadium wurde ex-situ mit Wasserstoff beladen.

Anschließend wurden mit einer Berkovichsonde mindestens 25 Eindrücke erzeugt. Dabei wird die Dehnrate $\dot{\epsilon}$ konstant gehalten. Der Nanoindenter regelt die Kraft $P$ so, dass $\dot{\epsilon}=\frac{\dot{P}}{P}=$ konst. gilt. Die Dehnrate wurde auf $\dot{\epsilon}=0,05 \mathrm{~s}^{-1}$ gesetzt und die Verformung wurde bis zur voreingestellten Endtiefe erhöht. Anschließend wird für 10 Sekunden die Kraft gehalten. Danach wird die Berkovichsonde bis auf 10\% der Maximalkraft zurückgezogen. Dieser letzte Schritt dient zur Bestimmung des Drifts, um entsprechende Korrekturen durchführen zu können.

Neben der Aufnahme der Kraft-Eindringkurven werden nach der Verformung die Probenoberflächen mit einem AFM abgebildet, um die Eindrucksstellen genauer charakterisieren zu können.

\footnotetext{
${ }^{19}$ Institut für Materialphysik, Universität Göttingen
} 


\subsection{Untersuchung der Versetzungsentwicklung in Molekulardynamiksimula- tionen von Nanoindentationsexperimenten}

Im vorherigen Abschnitt wurde erläutert, wie die Defektbildungsenergie und Versetzungsinteraktion experimentell untersucht werden können. Jedoch können so keine Aussagen zu den genauen Vorgängen auf der Ebene der Versetzungen gemacht werden. Eine Methode, um mögliche Versetzungsbewegungen darzustellen ist die Simulation der einzelnen Atombewegungen bei einem Nanoindentationsversuch. Da aufgrund von begrenzten Rechenkapazitäten keine Simulation möglich ist, welche den gleichen Bedingungen unterliegt, wie in einem Experiment, kann das Ziel dieser Untersuchungen nur sein eine generelle Vorstellung der Vorgänge zu erhalten.

Für die Simulation eines Nanoindentationsversuchs gibt es verschiedene Ansätze [134, 150-152], in dieser Arbeit wird die Spitze des Nanoindenters als Kugel mit einem Radius von $R_{S}=10 \mathrm{~nm}$ simuliert. Ein Atom erfährt eine Kraft, wenn dieses in die Spitze eingedrungen ist. Für diese Kraft $F_{S}$ wird folgendes Potential in Abhängigkeit des Abstandes von Spitzenmitte zum Atom $r_{i S}$ festgelegt:

$$
\overrightarrow{F_{S}}\left(\overrightarrow{r_{i S}}\right)=K \frac{\overrightarrow{r_{i S}}}{\left\|\overrightarrow{r_{i S}}\right\|} \exp \left(\left(R_{S}-\left\|\overrightarrow{r_{i S}}\right\|\right)^{3}\right)
$$

Die Konstante $K$ wurde auf 1 gesetzt. Die Nanoindentierung erfolgt, indem in jedem MD-Schritt die Position der Indenterspitze mit einer Geschwindigkeit von $10 \frac{\mathrm{m}}{\mathrm{s}}$ in die Oberfläche des simulierten Kristalls gefahren wird ${ }^{20}$.

Aufgrund der hohen Geschwindigkeiten des Indenters wurden weitere Simulationen durchgeführt, um den Einfluss von Wasserstoff zu veranschaulichen. Dazu wurden weitere Simulationsschritte eingefügt, bei denen sich nur der Wasserstoff bewegt. Dies gibt dem Wasserstoff mehr Zeit auf Spannungsfelder zu reagieren.

Die Simulationen wurden mit einem Zeitschritt von $\Delta t=1 \mathrm{fs}$ durchgeführt unter der Verwendung von Potentialen von Zhou et. al. [139] für das Palladium-Wasserstoffsystem und von Kimizuka et. al. [153] bzw. Wen et. al. [136] für das Eisen-Wasserstoffsystem ${ }^{21}$ Für die Oberfläche, die durch den Indenter verformt wird (Ebenennormale in Y-Richtung), wird eine freie Oberfläche verwendet. Für die gegenüberliegende Oberfläche wird als Randbedingung festgelegt, dass sich die Atome in der Ebene (X-,Z-Richtung) frei bewegen können, jedoch nicht in die Y-Richtung. Dies dient der Simulation eines unendlich ausgedehnten Kristalls in Indentationsrichtung und bewahrt die simulierten Atome vom Wegdriften. In der X-Z-Ebene werden periodische Randbedingungen verwendet. Des Weiteren wurden Leerstellen ins Material durch entfernen einzelner Atome eingebracht. Bevor die Nanoindentierung erfolgte wurden 5000 Schritte zum Equilibrieren durchgeführt.

Für die graphische Darstellung wurde das Programm Ovito [154] verwendet. In der Tabelle 1 sind die durchgeführten Simulationen mit den verwendeten Simulationsparameter dargestellt.

\footnotetext{
${ }^{20}$ Typische Geschwindigkeiten im Experiment sind $100 \frac{\mathrm{nm}}{\mathrm{s}}$. Damit folgt, dass die Ergebnisse der Simulation nur eine Veranschaulichung der Vorgänge beim Nanoindentieren erlauben.

${ }^{21}$ Die Simulation von Vanadium war nicht möglich, da kein passendes Potential in der Literatur gefunden werden konnte. Da das Eisen-Wasserstoffsystem bereits häufig durch Molekulardynamiksimulationen untersucht wurde, wird dieses System als Beispiel für krz Metall-Wasserstoffsysteme verwendet.
} 


\begin{tabular}{|c|c|c|c|}
\hline Metall und $\mathrm{H}-$ Konzentration $[\mathrm{H} / \mathrm{M}]$ & $\begin{array}{l}\text { Orientierung } \\
(\mathrm{x} ; \mathrm{y} ; \mathrm{z})[\mathrm{nm}] / \mathrm{Anz}\end{array}$ & $\begin{array}{l}\text { Größe } \\
\text { Atome }\end{array}$ & Besonderheiten \\
\hline$\overline{\mathrm{Pd} 0 \mathrm{H} / \mathrm{Pd}}$ & $\begin{array}{l}\text { kfz-}(011) ; \quad(22,0 \\
21,8) ; 627190\end{array}$ & 19,2 & \\
\hline $\mathrm{Pd} 0.0025 \mathrm{H} / \mathrm{Pd}$ & $\begin{array}{l}\text { kfz- }(011) ; \quad(22,0 \\
21,8) ; 628758\end{array}$ & 19,2 & \\
\hline $\mathrm{Pd} 0.0025 \mathrm{H} / \mathrm{Pd}$ & $\begin{array}{l}\text { kfz-(011); } \quad(22,0 \\
21,8) ; 628758\end{array}$ & 19,$2 ;$ & Extra Schritte für $\mathrm{H}$ \\
\hline $\mathrm{Fe} 0 \mathrm{H} / \mathrm{Fe}$ & $\begin{array}{l}\text { krz- }(001) ; \quad(21,7 \\
21,7) ; 635350\end{array}$ & 18,6 ; & Kimizuka et. al. [153] \\
\hline $\mathrm{Fe} 0 \mathrm{H} / \mathrm{Fe}$ & $\begin{array}{l}\text { krz- }(001) ; \quad(21,7 \\
21,7) ; 635350\end{array}$ & 18,6 ; & Wen et. al. [136] \\
\hline $\mathrm{Fe} 0.0025 \mathrm{H} / \mathrm{Fe}$ & $\begin{array}{l}\text { krz- }(001) ; \quad(21,7 \\
21,7) ; 636918\end{array}$ & 18,6 & Wen et. al. [136] \\
\hline
\end{tabular}

Tab. 1 Übersicht über die durchgeführten Simulationen. Damit der Wasserstoff auf Spannungsfelder reagieren kann, wurden bei einem Versuch zusätzliche MD-Schritte für Wasserstoff eingefügt, bei denen sich nur der Wasserstoff bewegt. Dabei folgten auf 100 normale Schritte 10000 Schritte für die Wasserstoffbewegung. Des Weiteren wurde das Eisen-Wasserstoffsystem mit Hilfe von zwei verschiedenen Potentialen untersucht. 


\subsection{Untersuchungen von Defekten in Wasserstoffatmosphäre im ETEM}

Ein ETEM (siehe Kapitel 3.3) erlaubt es, den Einfluss von Wasserstoff auf Versetzungen direkt zu sehen. Durch die Verwendung eines Zughalters (siehe Abbildung 22) kann das Zusammenspiel von mechanischen Spannungen und gelöstem Wasserstoff auf Versetzungen und Risse untersucht werden. Daher war das Ziel dieser Untersuchungen die Bewegung und die Interaktion von Versetzungen unter Wasserstoffeinfluss zu beobachten.

Für Untersuchungen dieser Arbeit wurden Palladium und Vanadium verwendet. Die Proben wurden zunächst aus ca. $80 \mu \mathrm{m}$ dicken Blechen geschnitten, danach wurde in die Mitte der Probe eine Mulde hinein geschliffen. Um die Versetzungsdichte zu reduzieren, wurden die Proben danach ausgelagert. Anschließend wurden die Vanadiumproben mit Palladium beschichtet, damit eine Wasserstoffbeladung ungehindert von der Oxidschicht erfolgen kann. Im letzten Schritt wurden die Proben elektronentransparent geätzt, wie in Kapitel 3.1.4 beschrieben. Die sich so ergebende Probengeometrie ist in Abbildung 22 dargestellt.

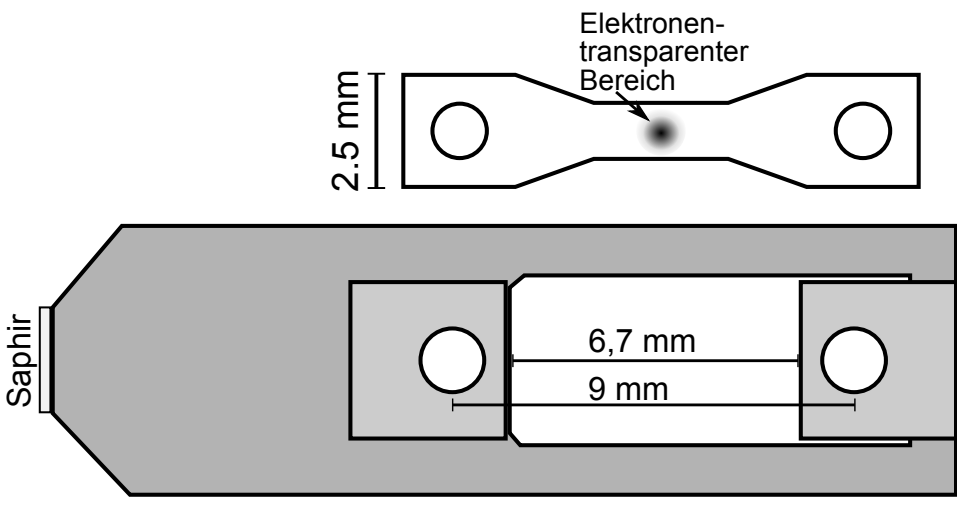

Abb. 22 Schematische Darstellung der verwendeten Probengeometrie (oben) und des Zughalters (unten).

Durch die Probengeometrie und durch die ungleichmäßigen Ränder des elektronentransparenten Bereichs ist es nicht möglich, eine definierte mechanische Spannung vorzugeben. Daher wurde ein Zughalter gewählt, welcher Längenänderungen der Probe definiert durchführen kann, jedoch keine Kraftmessungen ermöglicht. Dieser Halter zeichnet sich jedoch durch einen geringen Drift $^{22}$ im Vergleich zu anderen TEM-Zughaltern aus.

Um eine Vergleichbarkeit zwischen verschiedenen Proben zu ermöglichen, kann ein definierter Spannungszustand wie folgt erzeugt werden: Die Proben werden zunächst mit dem Zughalter gedehnt bis Versetzungen anfangen sich zu bewegen. Wenn diese Versetzungsbewegung abgeschlossen ist, befindet sich die Probe in einem Spannungszustand, in dem kleinste zusätzliche Spannungen wieder zur Bewegung von Versetzungen führen würden. Der beobachtete Bereich ist also mit einer Spannung nahe der Fließgrenze belastet. Anschließend wird der Wasserstoffpartialdruck in kleinen Schritten erhöht, hierbei wird die Schrittweite anhand des Sievertsschen Gesetzes (vgl. Kapitel 2.2 bzw. Gleichung 2.15) variiert um bekannte Konzentrationen einzustellen.

\footnotetext{
${ }^{22}$ Drift bezeichnet die Bewegung der Probe aus dem Beobachtungsbereich raus.
} 


\subsection{Verformungsexperimente an Vanadium-Mikrosäulen}

Die bisher beschriebenen Versuche untersuchen einzelne oder wenige Versetzungen. Die Verformung von Säulen erlaubt es, die Entstehung vieler Versetzungen unter genau bekannten experimentellen Voraussetzungen zu studieren. Dabei stellt die Untersuchung von Säulen im Mikrometerbereich den Übergang einzelner Versetzungen zu makroskopischen Verformungsverhalten dar.

Zur Durchführung von Säulendruckversuchen an Vanadium wird ein Vanadiumeinkristall verwendet. Die Oberfläche wird zunächst nach der Beschreibung in Kapitel 3.1.2 präpariert. Bevor in das Vanadium mit einem FIB die Säulen hinein geschnitten werden (siehe Kapitel 3.1.3), wird das Vanadium mit Wasserstoff mit verschiedenen Wasserstoffkonzentrationen in der $\alpha$-Phase des Vanadium-Wasserstoffsystems beladen. Nach dem Schneiden der Säulen werden diese im Nanoindenter verformt. Dazu wurden die Säulen mit einer Belastungsrate von $250 \mu \mathrm{N} / \mathrm{s}$ bis zu einer Solltiefe von $1000 \mathrm{~nm}$ verformt und anschließend mit doppelter Entladungsrate entlastet. Dabei wurden die Kraft und die Eindringtiefe des Indenters gemessen. Des Weiteren wurden REMAufnahmen von den Säulen vor und nach dem Verformen angefertigt. Dies ermöglicht die Ermittlung der Höhe der jeweiligen Säule für die Bestimmung der technischen Dehnung und der technischen Spannung. Besondere Aufmerksamkeit bei diesen Experimenten wird der Oberflächenmorphologie der Säulen gewidmet.

\subsection{Untersuchungen zur Bildung von Versetzungsnetzwerken am Beispiel von Palladium-Wasserstoff Legierungen}

Eine typische Verformungsmethode in industriellen Anwendungen ist das Walzen von Blechen. Das Kaltwalzen (siehe Kapitel 3.2.2) stellt eine einfache Methode dar, mit der eine hohe Verformungsrate der Probe aufgezwungen werden kann und damit eine hohe Defektdichte generiert werden kann. Um den Einfluss von Wasserstoff auf die Bildung von Versetzungsnetzwerken bei Verformung zu studieren, wurde Palladium bei verschiedenen Wasserstoffkonzentrationen, wie in Kapitel 3.2.2 beschrieben, präpariert und gewalzt.

Die so erhaltenden Palladiumbleche wurden in Zusammenarbeit mit Hans Peter Barth ${ }^{23}$ und Dr. Yuzeng Chen ${ }^{24}$ untersucht. Der Schwerpunkt in dieser Arbeit lag auf der Untersuchung der Mikrostruktur. Dazu wurden aus den Blechen TEM-Proben präpariert, um die Anordnung und Struktur von Versetzungen, bzw. Versetzungsnetzwerken zu untersuchen. Die Präparation einer TEM-Probe aus Palladium kann im Kapitel 3.1.4 nachvollzogen werden.

\footnotetext{
${ }^{23}$ Institut für Materialphysik, Univeristät Göttingen

${ }^{24}$ State Key Lab of Solidification Processing, Northwestern Polytechnical University, Xián, People's Republic of China
} 


\subsection{Bestimmung des Einflusses von Wasserstoff auf Palladium bei extremer Verformung}

Um eine maximale Verformung einer Probe zu erreichen, kann die HPT-Methode (siehe Kapitel 3.2.3) verwendet werden. Ziel dieser Untersuchungen ist es bei einem Höchstmaß an Verformung den zusätzlichen Einfluss von Wasserstoff auf die Verformung zu zeigen.

Für die Untersuchungen wurde ein $500 \mu$ m dickes Palladiumblech mit einer Reinheit von 99,5\% der Firma Wieland verwendet. Aus diesem Blech wurden mittels Funkenerosion Proben mit einem Durchmesser von $8 \mathrm{~mm}$ geschnitten und anschließend in verdünnter Salpetersäure gereinigt, um Rückstände der Funkenerosion zu entfernen. Diese Proben wurden danach bei $900^{\circ} \mathrm{C}$ im Vakuum $\left(p \approx 10^{-7}\right.$ mbar) für 48 Stunden ausgelagert.

HPT-Verformungen an der Universität Wien wurden von Dr. Y. Chen ${ }^{25}$ und Dr. D. Setman ${ }^{26}$ durchgeführt. Direkt vor der Verformung wurde Wasserstoff elektrolytisch in der Probe gelöst um Konzentrationen in der $\alpha$-Phase und im Zweiphasengebiet $\left(\alpha+\alpha^{\prime}\right)$ zu erreichen. Nach der Verformung wurden die Proben bis zur weiteren Verwendung in flüssigem Stickstoff gelagert. Für die Untersuchungen wurden die Proben in mehrere Teile aufgeteilt: Ein Teil wurde von Dr. D. Setman ${ }^{26}$ mittels DSC (engl. Differential Scanning Calorimetry) untersucht. Aus einem anderen Teil wurde im Rahmen dieser Arbeit TEM-Proben angefertigt und untersucht. Die Ergebnisse sind im Kapitel 5.2.3 dargestellt.

\footnotetext{
${ }^{25}$ State Key Lab of Solidification Processing, Northwestern Polytechnical University, Xián, People's Republic of China

${ }^{26}$ Groups Physics of Nanostructured/Functional Materials, Universität Wien
} 


\section{Ergebnisse}

Im Rahmen dieser Arbeit wurden verschiedenste Experimente zur Untersuchung des Einflusses von Wasserstoff auf die Bildung von Defekten in Metallen durchgeführt. Allen Experimenten ist gemeinsam, dass Metallproben mit oder ohne Wasserstoff verformt wurden. Die Art und Weise der Verformung wurde hierbei so gewählt, dass verschiedene Aspekte der Defektbildung untersucht werden konnten.

Durch Nanoindentation ist es möglich, den Effekt von Wasserstoff auf die Bildung einzelner Versetzungen zu untersuchen. Die Reaktion von Versetzungen unter Zugspannung auf Wasserstoffgas ermöglicht es, einzelne Versetzungen im ETEM zu betrachten. Hingegen kann beim Walzen von Palladiumproben oder der Verformung durch High Pressure Torsion der Effekt von Wasserstoff auf die Bildung vieler Versetzungen und deren Interaktion bzw. die Entstehung von Korngrenzen beobachtet werden. Mikrosäulendruckversuche stellen ein mittleres Regime dar. Hierbei wird die Verformung zum einen von der Bildung einzelner Versetzungen und zum anderen, falls die Versetzungen im Material verbleiben, von der Interaktion der Versetzungen miteinander beeinflusst. Das Ziel der Untersuchungen war es, die Wirkung von Wasserstoff auf diese Verformungsarten an sich und die Abhängigkeit von der Konzentration des eingelagerten Wasserstoffs im Besonderen $\mathrm{zu}$ verstehen und zu quantifizieren.

\subsection{Einfluss von Wasserstoff auf einzelne Defekte}

\subsubsection{Einfluss von Wasserstoff auf den Pop-In Effekt bei Nanoindentierung}

Wird ein einkristallines Material mit einer spitzen Sonde (Spitzenradius typischerweise $\leq 100 \mathrm{~nm}$ ) indentiert, zeigt die Kraft-Dehnungskruve häufig einen sog. Pop-In, siehe Kapitel 3.2.1. Im Folgenden werden die Ergebnisse zu den Nanoindentationsversuchen an Vanadium und Palladium vorgestellt. Dabei wird auf den Einfluss von Wasserstoff auf die Kraft-Eindringkurven und auf die Morphologie der Oberfläche eingegangen.

\section{Nanoindentationsversuche an Vanadium}

In Abbildung 23 sind die Ergebnisse zu den Versuchen an Vanadium zusammenfassend dargestellt. Die AFM-Aufnahmen zeigen zwei Indentationseindrücke. Aufgrund der steilen Kanten kann die wahre Tiefe des Eindrucks nicht bestimmt werden. Die Höhenskala orientiert sich daher an der Oberfläche. Die linke Seite zeigt einen Versuch ohne Wasserstoff, die rechte einen Versuch mit einer Konzentration von $0,03 \mathrm{H} / \mathrm{V}$. Es ist zu erkennen, dass die Wölbungen neben dem Loch des Eindrucks, der sog. Pile-up, beim Versuch mit Wasserstoff deutlich größer sind als beim Versuch ohne Wasserstoff.

Die Kraft-Eindringkurven zeigen zwei Pop-Ins, erkennbar durch einen waagerechten Abschnitt in den Kraft-Eindringkurven. Der erste Pop-In (durch 1 markiert) bei ca. $10 \mu \mathrm{N}$ ist unabhängig von der Wasserstoffkonzentration. Beim zweiten Pop-In (durch 2 bzw. 3 markiert) ist erkennbar, dass die Kraft (engl. Pop-In Load), bei der der Pop-In Effekt auftritt, bei der Probe, welche mit einer Konzentration von $0,03 \mathrm{H} / \mathrm{V}$ untersucht wurde, deutlich niedriger ist.

In Abbildung 24 ist die Kraft, bei der dieser Pop-In auftritt, gegen die Wasserstoffkonzentration dargestellt. Hierzu wurde die Kraft in der Mitte des waagerechten Abschnitt der KraftEindringkurven genommen und für eine Konzentration gemittelt. Es ist zu erkennen, dass die Kraft, bei der der Pop-In Effekt $P_{C}$ auftritt, zwischen den Wasserstoffkonzentrationen 0,0075 und $0,0150 \mathrm{H} / \mathrm{V}$ reduziert wird. 


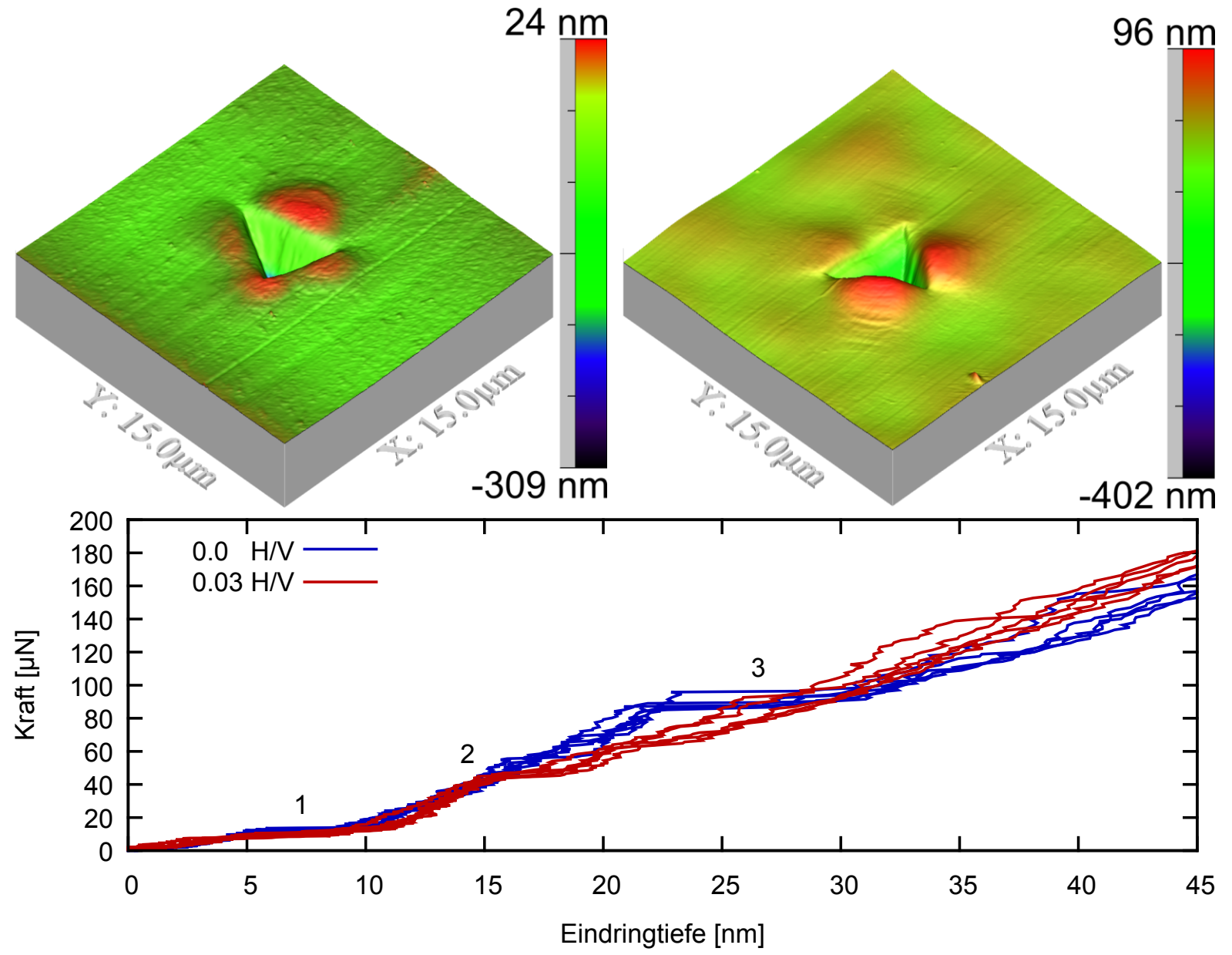

Abb. 23 AFM-Abbildungen von Berkovichsonden-Eindrücken in Vanadium. Oben links: Eindruck in unbeladenem Vanadium, oben rechts: Eindruck in mit 0,03 H/V beladenem Vanadium. Die AFM-Bilder zeigen, dass ein Pile-up entsteht, jedoch mit Wasserstoff ist dieser erheblich größer. Die Kraft-Eindringkurven (unten) zeigen jeweils zwei Pop-Ins.

Die Kraft beim Pop-In 1 ist unabhängig von der Wasserstoffkonzentrationen und tritt bei ca. $10 \mu \mathrm{N}$ auf. Mit gelöstem Wasserstoff in der Probe ist die Kraft des Pop-Ins 2 geringer als ohne Wasserstoff (Pop-In 3).

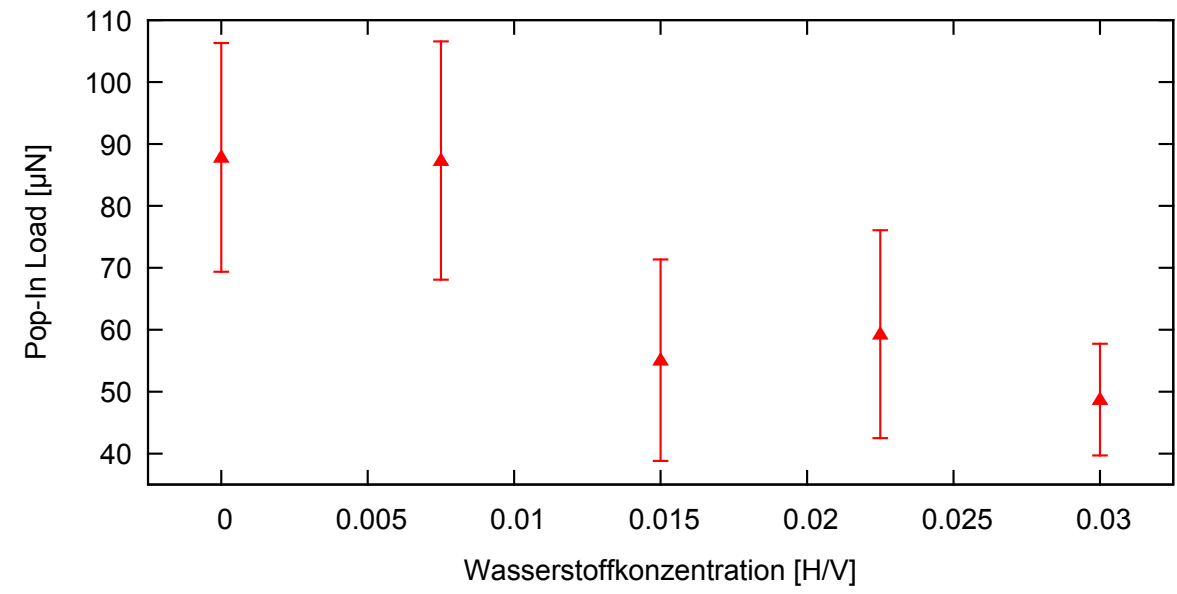

Abb. 24 Entwicklung der Kraft, bei der der Pop-In Effekt auftritt, gegen die Wasserstoffkonzentration während der Nanoindentierung. 


\section{Nanoindentationsversuche an Palladium}

In Abbildung 25 sind die entsprechenden Versuchsergebnisse zur Nanoindentierung von wasserstofffreiem und mit 0,005 H/Pd beladenem Palladium dargestellt. Bei diesen Versuchen wurde eine polykristalline Palladiumprobe verwendet. Damit eine Vergleichbarkeit gewährleistet werden kann, wurde ein und das selbe Korn, welches einen Durchmesser von ca. $300 \mu \mathrm{m}$ hatte, für alle Versuche verwendet. Im Gegensatz zu Vanadium ist hier kein Unterschied im Pile-up des zurückbleibenden Eindrucks zu erkennen. Die nicht indentierte Oberfläche bleibt nahezu unverändert. Jedoch zeigen die Kraft-Eindringkurven ein zum Vanadium vergleichbares Verhalten und die Kraft zum Erzeugen des Pop-In Effekts wird auch hier mit Wasserstoff niedriger. Dieser Effekt scheint jedoch nicht so ausgeprägt zu sein wie beim Vanadium.

Für die Kraft $P_{C}$, bei der der Pop-In Effekt auftritt, ergeben sich folgende Werte:

$$
\begin{aligned}
& P_{C, 0,000 H / P d}=68 \pm 7 \\
& P_{C, 0,005 H / P d}=48 \pm 9
\end{aligned}
$$

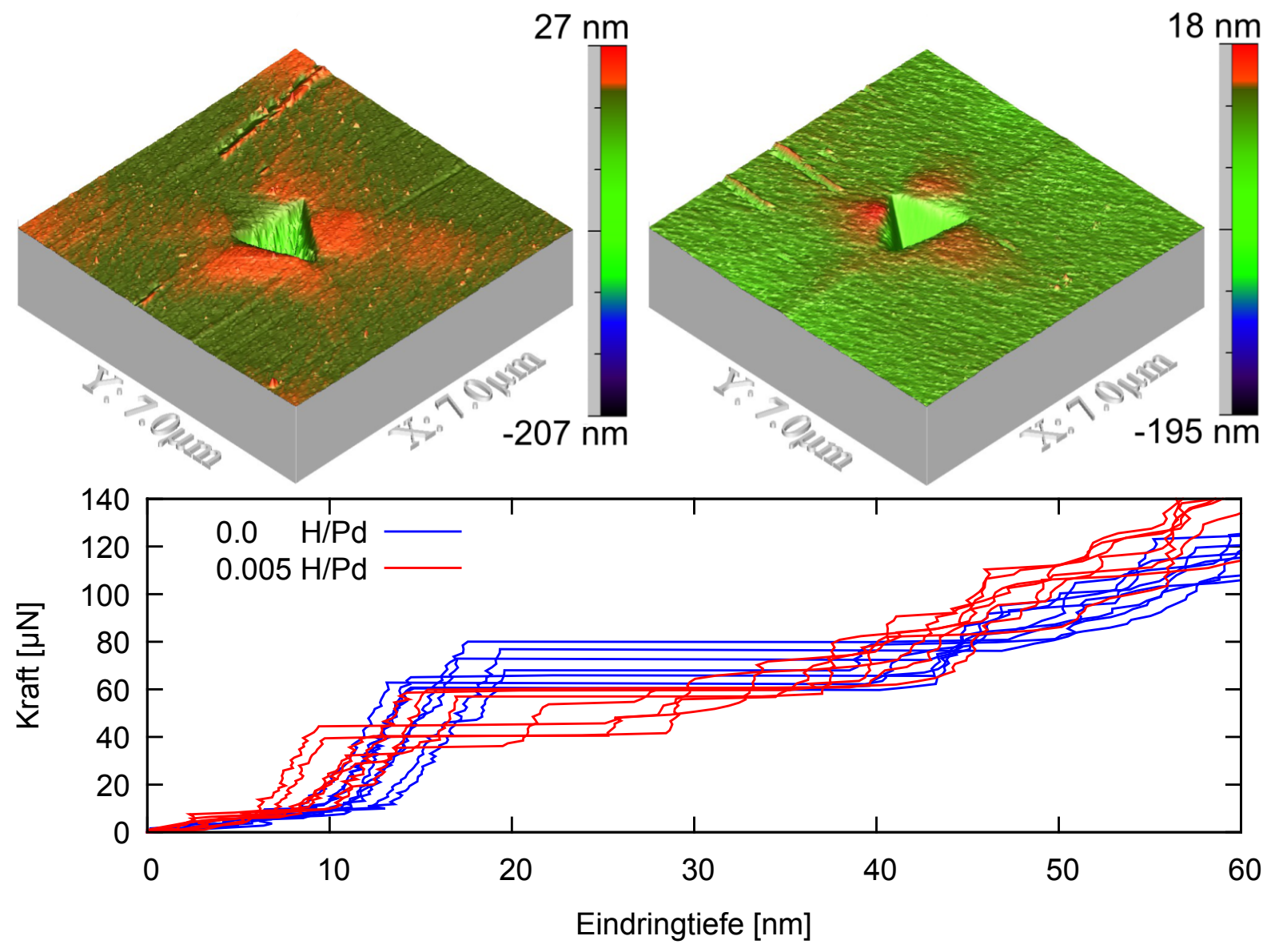

Abb. 25 AFM-Abbildungen von Berkovichsonden-Eindrücken in Palladium. Oben links: Eindruck in unbeladenem Palladium, oben rechts: Eindruck in mit 0,005 H/Pd beladenem Palladium. Die AFM-Bilder zeigen, dass die nicht indentierte Oberfläche nahezu unverändert bleibt. Die Kraft-Eindringkurven (unten) zeigen, dass die Kraft, bei der der Pop-In Effekt auftritt, ein wenig niedriger ist, wenn Wasserstoff in Palladium gelöst ist. 


\subsubsection{Versetzungsentwicklung in MD-Simulationen von Nanoindentation}

Molekulardynamiksimulationen (MD) ermöglichen es, die Entstehung und Entwicklung von Versetzungen auf atomarer Skala zu veranschaulichen. Dazu wurde ein kfz Metall (Palladium) und ein krz Metall (Eisen) virtuell durch Nanoindentation verformt.

Die hier vorgestellten Ergebnisse sollen zur bildlichen Darstellung der Versetzungen und deren Interaktion im Metall beim Nanoindentieren dienen. Die Darstellung der Ergebnisse gliedert sich in zwei Teile: Zunächst wird die Bildung der Versetzungsstruktur gezeigt. Anschließend werden diese Vorgänge mit der Kraft-Eindringkurve verknüpft.

Zur Darstellung der Versetzungsstruktur wird die CNA Methode (engl. Common Neighbor Analysis) im Programm Ovito [154] verwendet. Diese Methode ermittelt für jedes Atom die Nachbarschaftsbeziehung und ermittelt dadurch die lokale Gitterstruktur. Werden alle Atome, welche sich in der Ausgangsstruktur (Palladium kfz; Eisen krz) befinden, entfernt bleiben nur die Atome zurück, die an einen Defekt grenzen oder Teil eines Defekts sind. Diese sind in diesen Simulationen neben der Oberfläche, Leerstellen und Versetzungen. Da sich Versetzungen im kfz Gitter in partielle Versetzungen aufteilen, sind auch Stapelfehler zu erkennen. Diese haben eine hcp Struktur.

Die Abbildung 26 dient zur Veranschaulichung der Darstellungsmethode. Bei der dargestellten Simulation wurde eine quaderförmige krz Eisen-Probe, in der Wasserstoff gelöst wurde, simuliert. Die Versetzungsstruktur wurde durch einen kugelförmigen Nanoindenter erzeugt, indem dieser in die Oberfläche hineingefahren wurde.
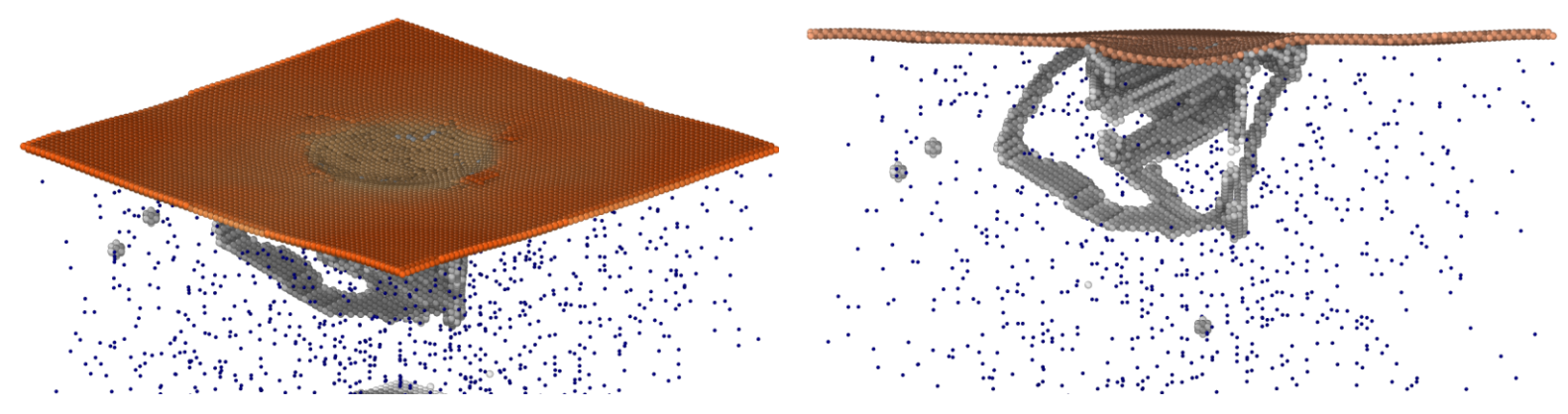

Abb. 26 Darstellung des Eindrucks eines Nanoindenters in einer in (001) orientierten Eisenoberfläche (braun). Links: Aufsicht auf den Eindruck (Blickrichtung in eine $\langle 012\rangle$-Richtung), Rechts: Blickrichtung aus einer $\langle 011\rangle$-Richtung, wobei die simulierte Probe senkrecht zur Blickrichtung durch den Eindruck aufgeschnitten wurde. Diese Simulation zeigt die Entstehung von Versetzungen (graue fadenartige Strukturen in der Mitte der Bilder) unterhalb eines Nanoindentationseindrucks. Neben den Versetzungen sind Wasserstoffatome (blau) und einzelne Leerstellen (zu erkennen an punktförmig angeordnete graue Atome) zu sehen. 


\section{Simulationen der Nanoindentation von Palladium-Wasserstoff}

In der Abbildungsserie 27 ist die Entstehung von Versetzungen in Palladium bei Nanoindentation gezeigt. Die Abbildungsserie 28 zeigt die entsprechende Serie von Palladium, in dem Wasserstoff mit einer Konzentration von $0,0025 \mathrm{H} / \mathrm{Pd}$ gelöst ist. Hierbei wurde eine (011) orientierte Oberfläche mit einer Nanoindentationsspitze mit einem Radius von $10 \mathrm{~nm}$ verformt, die Identationsgeschwindigkeit betrug $10 \mathrm{~m} / \mathrm{s}$.

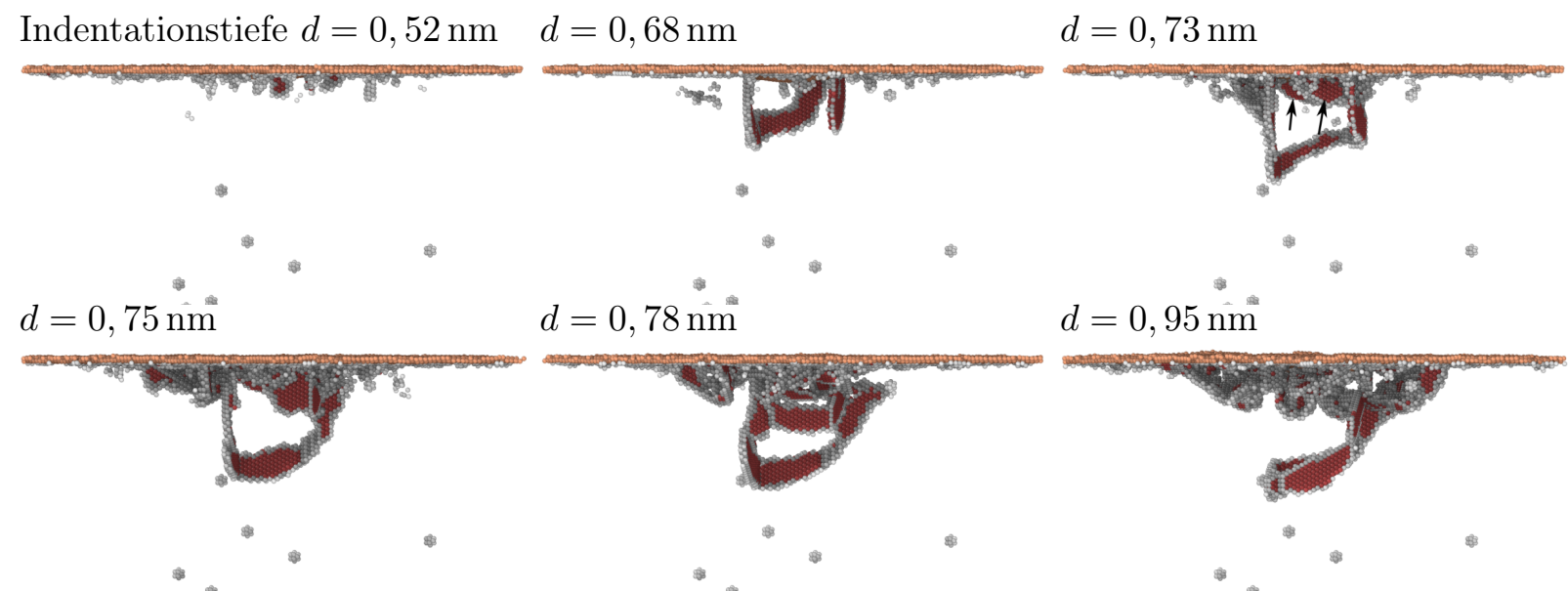

Abb. 27 Darstellung der Versetzungsstrukturen in Palladium mit einer in (011) orientierten Oberfläche bei Nanoindentationsversuchen, Blickrichtung in eine $\langle 111\rangle$-Richtung. Bei der Darstellung wurden alle Atome, welche sich in einer lokalen kfz Umgebung befinden entfernt.

Rot gefärbte Atome besitzen eine hcp Umgebung, d. h. sind Teil eines Stapelfehlers, grau gefärbte Atome konnten keiner eindeutigen Struktur zugeordnet werden. Die Nanoindentation wurde zentral auf die Oberfäche (obere braun gefärbte Atomschicht) ausgeführt (Indenter nicht gezeigt). Die Pfeile in der Abbildung bei $d=0,73 \mathrm{~nm}$ deuten auf die Bildung sekundärer Versetzungsringe hin.

Die wasserstofffreie Palladiumprobe zeigt, dass ab einer Eindringtiefe von 0,52 nm die ersten Stapelfehler an der Oberfläche entstehen, welche sich leicht versetzt zum Zentrum der Spitze befinden. Diese Stapelfehler sind umgeben von partiellen Versetzungen und befinden sich auf (111) Ebenen. Bei weiterer Verformung wachsen die Stapelfehler, wobei nur geringe Tiefenänderungen des Indenters benötigt werden. Ist ein Durchmesser von 3-6 Atomlagen, was 0.82-1.65 nm entspricht, erreicht, bildet sich eine abschließende partielle Versetzung im Inneren des Stapelfehlers und es entsteht ein Versetzungsring, vgl. dazu Abbildung 27 bei $d=0,68 \mathrm{~nm}$. Hier sind zwei Versetzungsringe gezeigt, der rechte befindet sich im Anfangsstadium und der linke ist weiter fortgeschritten. Ein Vergleich der Versetzungsstruckturen mit der Kraft-Eindringkurve (Abbildung 29) zeigt, dass der Pop-In Effekt genau mit der Bildung der ersten Versetzungsringe bei einer Tiefe von $d=0,65 \mathrm{~nm}$ zusammenfällt. Wird der Indenter weiter ins Material getrieben, bildet sich zunächst ein waagerechter Verlauf aus. Dies wird damit interpretiert, dass Versetzungen sich ungehindert ausbreiten können. Ab einer Tiefe von $d=0,73 \mathrm{~nm}$ (vgl. Abbildung 27) bilden sich sekundäre Versetzungsringe aus. Dies benötigt mehr Energie und diese neuen Versetzungen sind in ihrer Ausbreitung durch die schon vorhandenen Versetzungen eingeschränkt. Dies zeigt sich in der Kraft-Einringkurve durch das Ende des Pop-In Effektes.

Wie in Kapitel 3.4 beschrieben wurden zusätzlich Leerstellen ins Material eingebracht, diese scheinen jedoch die Versetzungsbildung nicht zu beeinflussen

In einer Simulation mit Wasserstoff, bei der der Wasserstoff zusätzliche Simulationsschritte durchläuft (Abbildung 28), zeigt sich ein ähnlicher Ablauf. Versetzungen liegen aufgespalten in partielle Versetzungen vor. Die dabei vorliegenden Stapelfehler bilden Ringe aus. Sind die ersten Versetzungen gebildet, können sich diese ungehindert ausbreiten. Es ergibt sich der Pop-In-Effekt. Werden sekundäre Versetzungen gebildet ist das Ende des Pop-In erreicht. 
Der Vergleich der beiden Simulationen zeigt, dass die Versetzungen im Fall der mit Wasserstoff simulierten Probe zunächst näher zur Oberfläche lokalisiert sind. Des Weiteren reichte die Simulationszeit im Fall der mit Wasserstoff simulierten Probe aus, damit sich ein, von der Oberfläche unabhängiger, Versetzungsring loslösen konnte.

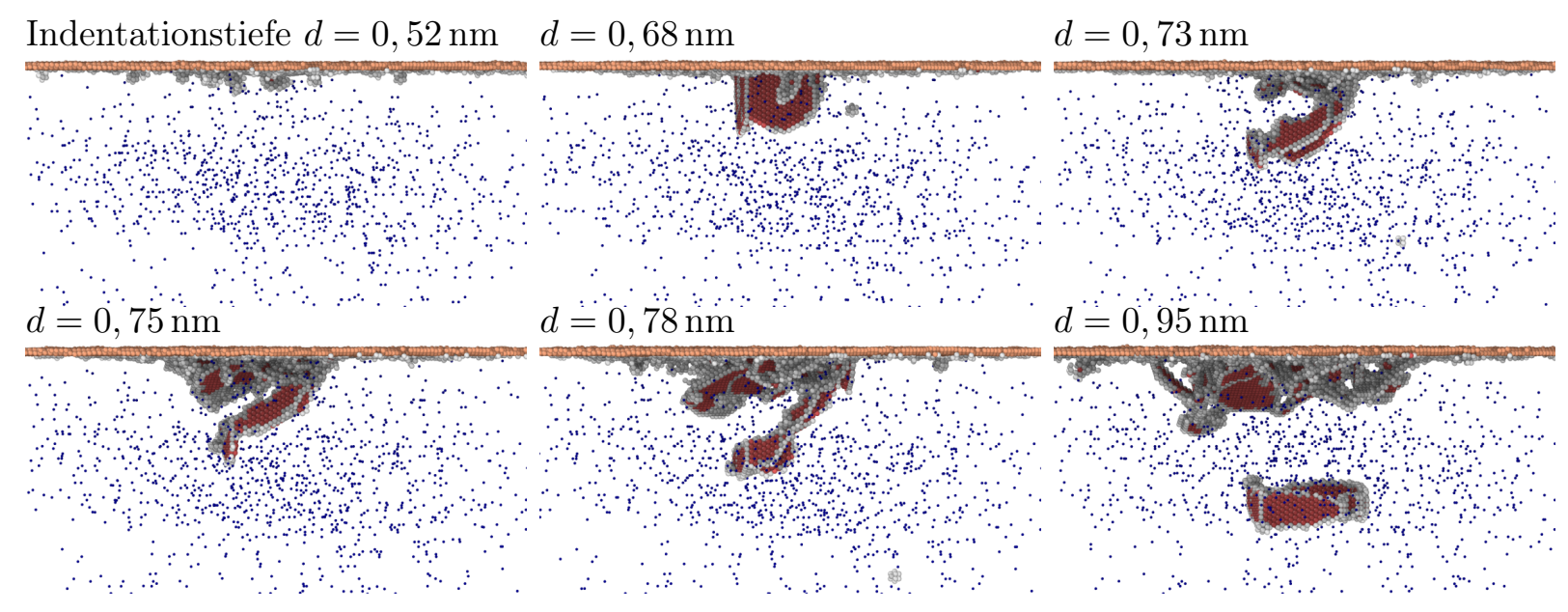

Abb. 28 Darstellung der Versetzungsstrukturen in Palladium, welches einen Wasserstoffgehalt von $0.0025 \mathrm{H} / \mathrm{Pd}$ hat, mit einer in (011) orientierten Oberfläche bei Nanoindentationsversuchen,

Blickrichtung in eine $\langle 111\rangle$-Richtung. Bei der Darstellung wurden alle Atome, welche sich in einer lokalen kfz Umgebung befinden entfernt. Blau gefärbte Atome sind Wasserstoffatome. Rot gefärbte Atome besitzen eine hcp Umgebung, $d$. h. sind Teil eines Stapelfehlers, grau gefärbte Atome konnten keiner eindeutigen Struktur zugeordnet werden. Die Nanoindentation wurde zentral auf die Oberfläche (obere braun gefärbte Atomschicht) ausgeführt (Indenter nicht gezeigt).

Abbildung 29 zeigt die bei der Simulation der Nanoindentation von Palladium aufgenommenen Kraft-Eindringkurve. Diese Kurven zeigen, das in Kapitel 4.1 beschriebene Verhalten. Bis zu einer Tiefe von ungefähr $d=0,6 \mathrm{~nm}$ folgt die Kraft-Eindringkurve einer Potenzfunktion der Form $F=a \cdot d^{b}(F$ : Kraft, $a$ und $b$ Fitparameter $)$. Danach gibt es leichte Abweichungen von der Potenzfunktion. Im Vergleich zu der Versetzungsstruktur fällt dies mit der Bildung der ersten Stapelfehler bei $d=0,52 \mathrm{~nm}$ zusammen. Ab ungefähr $d=0,65 \mathrm{~nm}$ folgt ein waagerechter Abschnitt. Dies Phänomen fällt mit der Bildung erster vollständiger Versetzungsringe zusammen.

Der weitere Verlauf der Kraft-Eindringkurven unterscheidet sich je nach Wasserstoffkonzentration und Simulationsmethode. Die Kurven der wasserstofffreien Simulation und der Simulation bei einer Konzentration von 0,0025 H/Pd, bei der zusätzliche Schritte für Wasserstoff eingefügt wurden, zeigen ein ähnliches Verhalten. Die Position des Endes des Pop-Ins ist hierbei unterschiedlich. Die Simulation mit Wasserstoff zeigt ein früheres Ende des Pop-Ins.

Der Vergleich der Kraft-Eindringkurven der Simulationen mit Wasserstoff zeigt, dass es entscheidend ist, ob genügend Zeit zum Diffundieren von Wasserstoff gegeben wird. Erst, wenn zusätzliche Schritte für Wasserstoff eingefügt werden, wird ein Verhalten vergleichbar zum Experiment beobachtet.

Um den Beginn des Pop-Ins genau zu bestimmen, wurden die Atome, welche nicht dem idealem Gitter entsprechen (und nicht Teil der Oberfläche sind) gezählt. Diese Anzahl spiegelt die Menge der Vesetzungen wieder. In Abbildung 29 zeigt sich, dass zu Beginn des Pop-Ins die Anzahl der Atome, welche nicht in einer idealen Gitterumbegung sind stark ansteigt und im weiteren Verlauf des Pop-Ins ungefähr gleich bleibt. Bei der Untersuchugun ohne Wasserstoff steigt die Anzahl nach dem Ende des Pop-Ins weiter an. Bei den Untersuchungen mit Wasserstoff ist dies nicht eindeutig zu erkennen. 

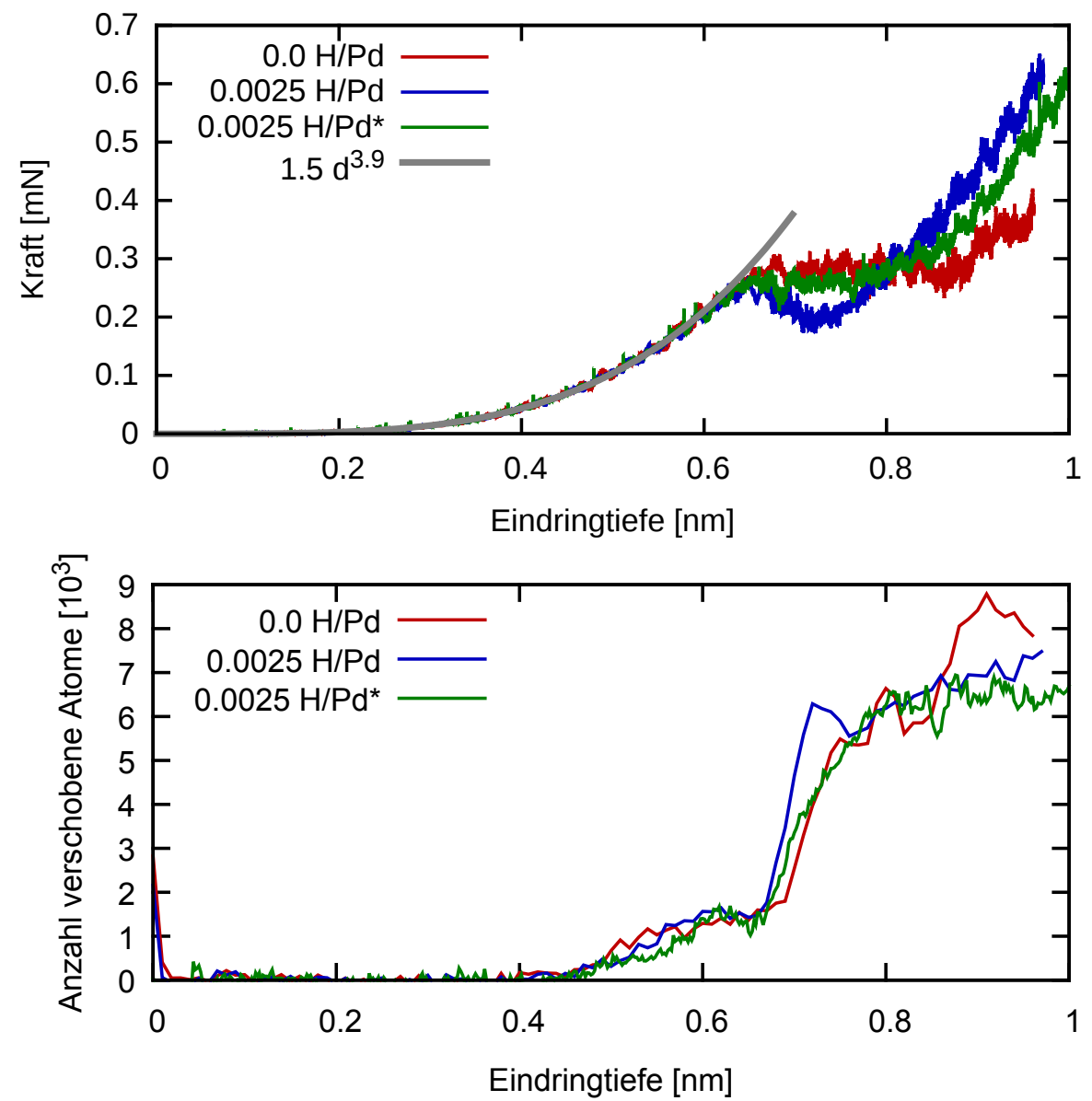

Abb. 29 Kraft-Eindringkurve von Palladium (oben), von (011)-orientierten Oberflächen für wasserstofffreies Palladium und Palladium mit einer Wasserstoffkonzentration von $0.0025 \mathrm{H} / \mathrm{Pd}$. Die untere Abbildung zeigt die Anzahl der Atome, welche nicht dem idealen Gitter entsprechen, also Versetzungen und Leerstellen, gegen die Eindringtiefe des Indenters. Bei den mit * gekennzeichneten Kurven wurden zusätzliche Schritte eingefügt, bei denen Wasserstoff unabhängig vom Palladium berechnet wurde, damit dieser besser auf Spannungsfelder reagieren kann. 


\section{Simulationen der Nanoindentation von Eisen-Wasserstoff}

Die Simulation von Eisen erwies sich als deutlich schwieriger. Daher wurden verschiedene in der Literatur gefundene Potentiale für das Eisen-Wasserstoff System untersucht.

In der Abbildungsserie 30 sind die Ergebnisse einer Simulation von Eisen mit dem Potential von Kimizuka et. al. [153] ohne Wasserstoff zu sehen. Beim Betrachten der Oberfläche fällt auf, dass diese im Verlauf der Simulation schwingt. Dieses Phänomen führt in der Kraft-Eindringkurve zu Schwingungen, da der Abstand zwischen Indenterspitze und Oberfläche unvorhersagbar variiert. Dieses Problem tritt bei allen untersuchten Potentialen auf und führt dazu, dass keine aussagekräftige Kraft-Eindringkurve für das Potential von Kimizuka et. al. [153] ermittelt werden konnte. Da jedoch die Versetzungsdynamik im Falle des Potentials von Kimizuka et. al. sichtbarer ist, werden diese Ergebnisse zur Darstellung benutzt. Die Ergebnisse, welche mit dem Potential von Wen et. al. [136] erzeugt wurden, werden im Anschluss behandelt.

In der Abbildungsserie 30 sind Zwischenschritte der Simulation unter Verwendung des Potentials von Kimizuka et. al. [153] gezeigt. Hierbei wurden alle Atome entfernt, welche sich in einer idealen krz Umgebung befinden. Es bleiben neben der Oberfläche und den Versetzungen Leerstellen zurück.

Bei der Nanoindentation entstehen zunächst an der Oberfläche Versetzungen, welche in den meisten Fällen eine lokalisierte Versetzungslinie aufweisen. Des Weiteren sind bereits beim Auftauchen der ersten Versetzungen an der Oberfläche $(d=0,70 \mathrm{~nm})$ größere Unregelmäßigkeiten unabhängig von der Oberfläche zu erkennen. Die Abbildung bei $d=0,73 \mathrm{~nm}$ zeigt, dass auch Versetzungen entfernt von dem Nanoindenter entstehen, welche jedoch im Verlauf der Simulation wieder verschwinden.

In den Abbildungen mit $d=0,80 \mathrm{~nm}$ bzw. $d=0,85 \mathrm{~nm}$ ist eine weitere Entstehungsmöglichkeit für Versetzungen zu erkennen. Am rechten Rand von der Abbildung mit $d=0,80 \mathrm{~nm}$ ist ein Versetzungsring zu erkennen. Der Vergleich mit der gleiche Position in der Abbildung mit $d=0,73 \mathrm{~nm}$ zeigt, dass hier zwei Leerstellen vorlagen. In der Abbildung mit $d=0,85 \mathrm{~nm}$ ist ein weiterer von der Oberfläche unabhängiger Versetzungsring zu erkennen. Auch hier zeigt ein Vergleich zum vorherigen Bild $(d=0,80 \mathrm{~nm})$, dass hier eine Leerstelle vorlag.

Einen weiteren Entstehungsmechanismus für Versetzungen ist in den Abbildungen mit $d=0,96 \mathrm{~nm}$ (Diese Zwischenschritte liegen nur 200 Simulationsschritte auseinander) zu sehen. Hier ist zentral eine Scheibe von Atomen zu erkennen. Ob es sich hierbei um einen Stapelfehler handelt, ist unklar. Bei der weiteren Entwicklung entstehen in diesem Fall zwei Versetzungsringe.

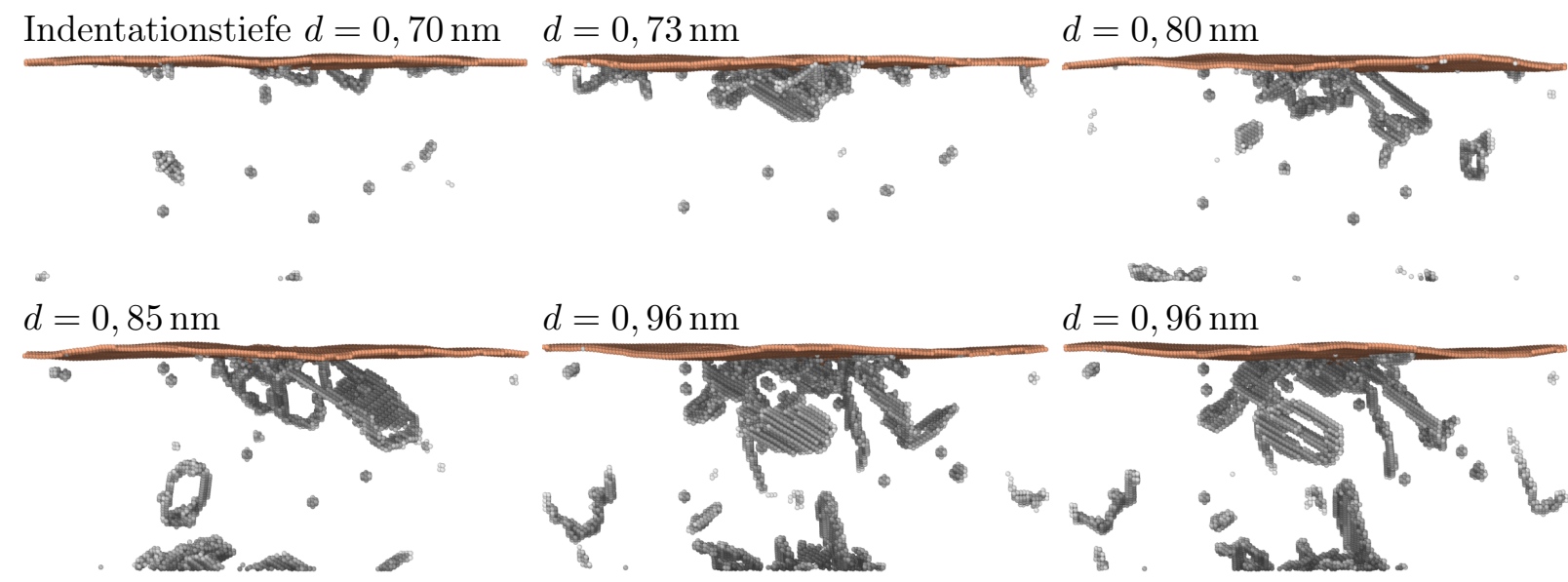

Abb. 30 Darstellung der Versetzungsstrukturen in Eisen, welche sich unter Verwendung des Potentials von Kimizuka et. al. [153] bei Nanoindentationsversuchen ergeben. Dabei ist die Oberfläche (001) orientiert, Blickrichtung in eine $\langle 011\rangle$-Richtung. Bei der Darstellung wurden alle Atome, welche sich in einer lokalen krz Umgebung befinden entfernt. Grau gefärbte Atome konnten keiner eindeutigen Struktur zugeordnet werden. $\mathrm{Zu}$ sehen sind Versetzungen und Leerstellen. Die Nanoindentation wurde zentral auf die Oberfläche (obere braun gefärbte Atomschicht) ausgeführt (Indenter nicht gezeigt). 
Die Abbildungsserie 31 zeigt die Entwicklung von Versetzungen in wasserstofffreiem Eisen unter der Verwendung des Potentials von Wen et. al. [136]. Es zeigt sich, dass Versetzungen zunächst unabhängig von der Oberfläche entstehen $(d=0,91 \mathrm{~nm}$ bis $d=0,93 \mathrm{~nm})$, in einem Abstand von $2,8 \mathrm{~nm}$ zwischen Zentrum der Versetzungsentstehung zur unveränderten Oberfläche. Wenn die Versetzungen die Oberfläche erreicht haben $(d=0,95 \mathrm{~nm})$ können nun auch Versetzungen an der Oberfläche nukleiert werden. Bei weiterer Nanoindentation nukleieren immer mehr Versetzungen, welche jedoch lokal unterhalb des Nanoindenters verbleiben. Dadurch kommt es zur Interaktion vieler Versetzungen. Erst bei fortgeschrittener Indentationstiefe bspw. bei $d=1,49 \mathrm{~nm}$ kommt es zu langreichweitiger Verformung des Materials.

Die Kraft-Eindringkurve (Abb. 33) zeigt für den Fall ohne Wasserstoff einen Pop-In Effekt. Dieser beginnt im Bereich von $d=0,9 \mathrm{~nm}$ und fällt somit mit der Entstehung der ersten Versetzung zusammen. Das Ende des Pop-Ins ist nicht eindeutig zu erkennen, jedoch steigt im Bereich um $d=0,95 \mathrm{~nm}$ die Kurve an. Die im Material nukleierten Versetzungen haben nun ihre maximale Ausbreitung erreicht, da die Oberfläche diese Versetzungen am weiteren Wachstum hindert, siehe Abbildung 31 bei $d=0,95 \mathrm{~nm}$.

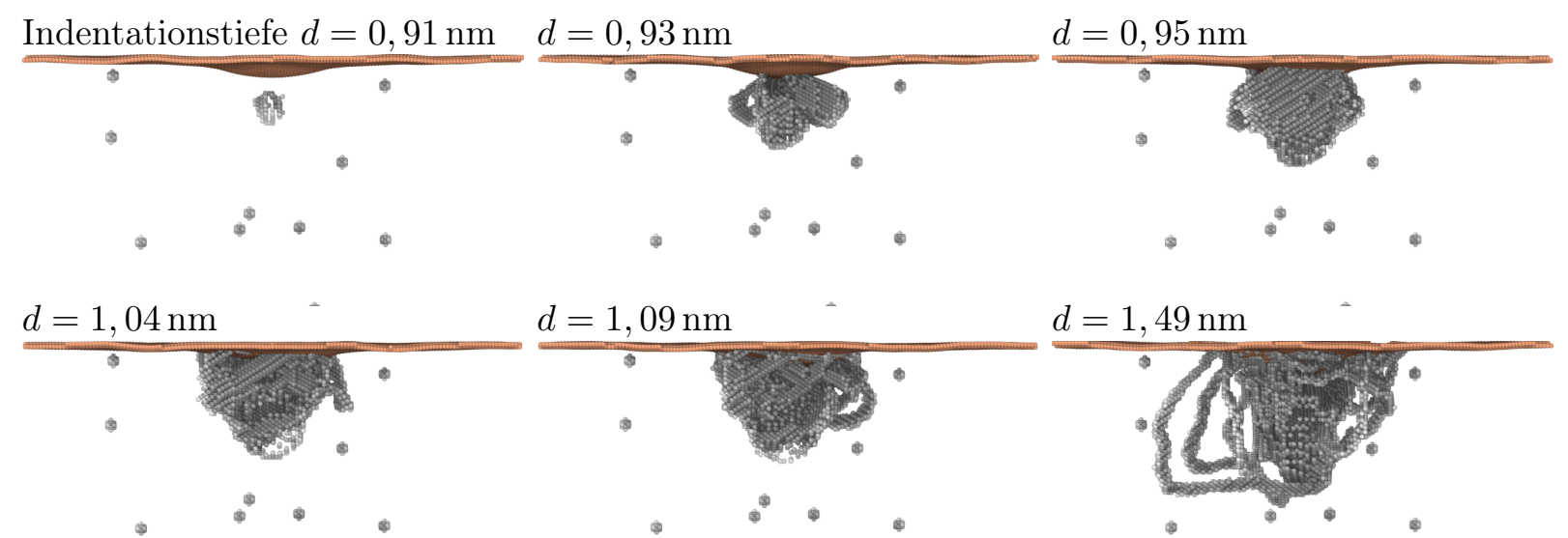

Abb. 31 Darstellung der Versetzungsstrukturen in Eisen (ohne Wasserstoff), welche sich unter Verwendung des Potentials von Wen et. al. [136] bei Nanoindentationsversuchen ergeben, Blickrichtung in eine $\langle 011\rangle$-Richtung. Dabei ist die Oberfläche (001) orientiert. Bei der Darstellung wurden alle Atome, welche sich in einer lokalen krz Umgebung befinden entfernt. Grau gefärbte Atome konnten keiner eindeutigen Struktur zugeordnet werden. Zu sehen sind Versetzungen und Leerstellen. Leerstellen sind anhand von vereinzelten Punkten zu erkennen. Die Nanoindentation wurde zentral auf die Oberfläche (obere braun gefärbte Atomschicht) ausgeführt (Indenter nicht gezeigt).

Bei der Simulation eines Nanoindentationsversuchs an Eisen mit Wasserstoff zeigen sich deutliche Unterschiede. In Analogie zum Palladium wurde versucht, zusätzliche Schritte für den Wasserstoff einzufügen. Dies führte jedoch (für alle untersuchten Potentiale) zur Bildung von Unregelmäßigkeiten (Bildung von kfz Gittern Ramasubramaniam et. al. [155], Bildung von Leerstellenansammlungen Kimizuka et. al. [153] oder wasserstofffreien unregelmäßig geordnete Strukturen Wen et. al. [136]) und wurde daher nicht weiter verfolgt.

In der Abbildungsserie 32 sind die Ergebnisse einer Simulation von Eisen mit einer Wasserstoffkonzentration von $0,0025 \mathrm{H} / \mathrm{Fe}$ unter Verwendung des Potentials von Wen et. al. zu sehen. Es zeigt sich, dass die Bildung von Versetzungen erst deutlich später einsetzt, als im Fall ohne Wasserstoff. Des Weiteren zeigt sich eine offenere Struktur, also eine geringere Häufung von Versetzungen unterhalb des Zentrums des Nanoindenters, vergleiche hierzu die Abbildungen mit $d=0.91 \mathrm{~nm}$ bis $d=1,04 \mathrm{~nm}$ in der Abbildungsserie 31, welche die gleiche Tiefenänderung des Nanoindenters aufweist, wie die gesamte Abbildungsserie 32.

Die Kraft-Eindringkurve 33 für diese Simulation unterliegt im Bereich der Versetzungsentstehung sehr großen Schwankungen, wodurch keine Aussagen zum Pop-In Verhalten möglich sind.

Analog zum Palladium wurden die Atome gezählt, welche nicht in der idealen Gitterstruktur sind. In Abbildung 33 zeigt sich, dass die Versetzungen in der Untersuchung ohne Wasserstoff deutlich 
früher entstehen, als in der Untersuchung mit Wasserstoff. Bei der Untersuchung ohne Wasserstoff ist zudem zu erkennen, dass die Anzahl, nachdem die ersten Versetzungen gebildet worden sind, nahezu linear mit der Indentertiefe zunimmt.

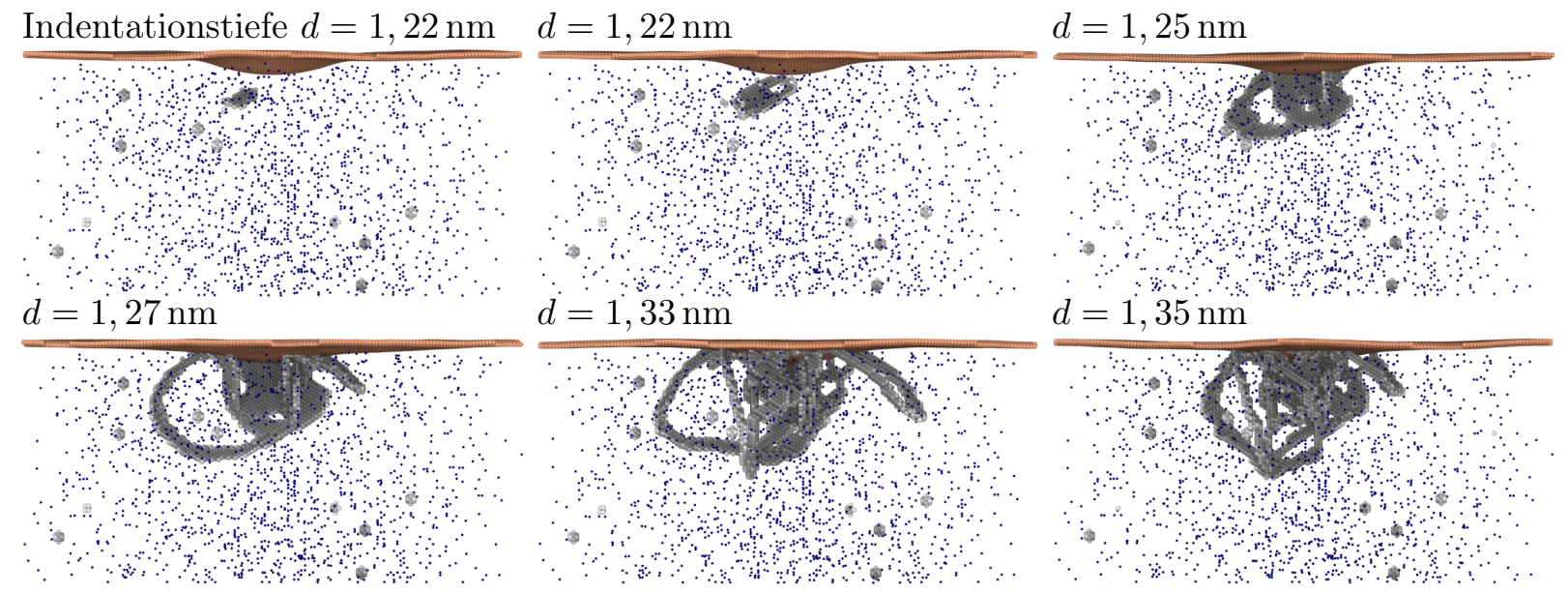

Abb. 32 Darstellung der Versetzungsstrukturen in Eisen (mit einer Konzentration von $0,0025 \mathrm{H} / \mathrm{Fe}$ ), welche sich unter Verwendung des Potentials von Wen et. al. [136] bei Nanoindentationsversuchen ergeben, Blickrichtung in eine $\langle 011\rangle$-Richtung. Dabei ist die Oberfläche (001) orientiert. Bei der Darstellung wurden alle Atome, welche sich in einer lokalen krz Umgebung befinden entfernt. Grau gefärbte Atome konnten keiner eindeutigen Struktur zugeordnet werden. Die Nanoindentation wurde zentral auf die Oberfläche (obere braun gefärbte Atomschicht) ausgeführt (Indenter nicht gezeigt).
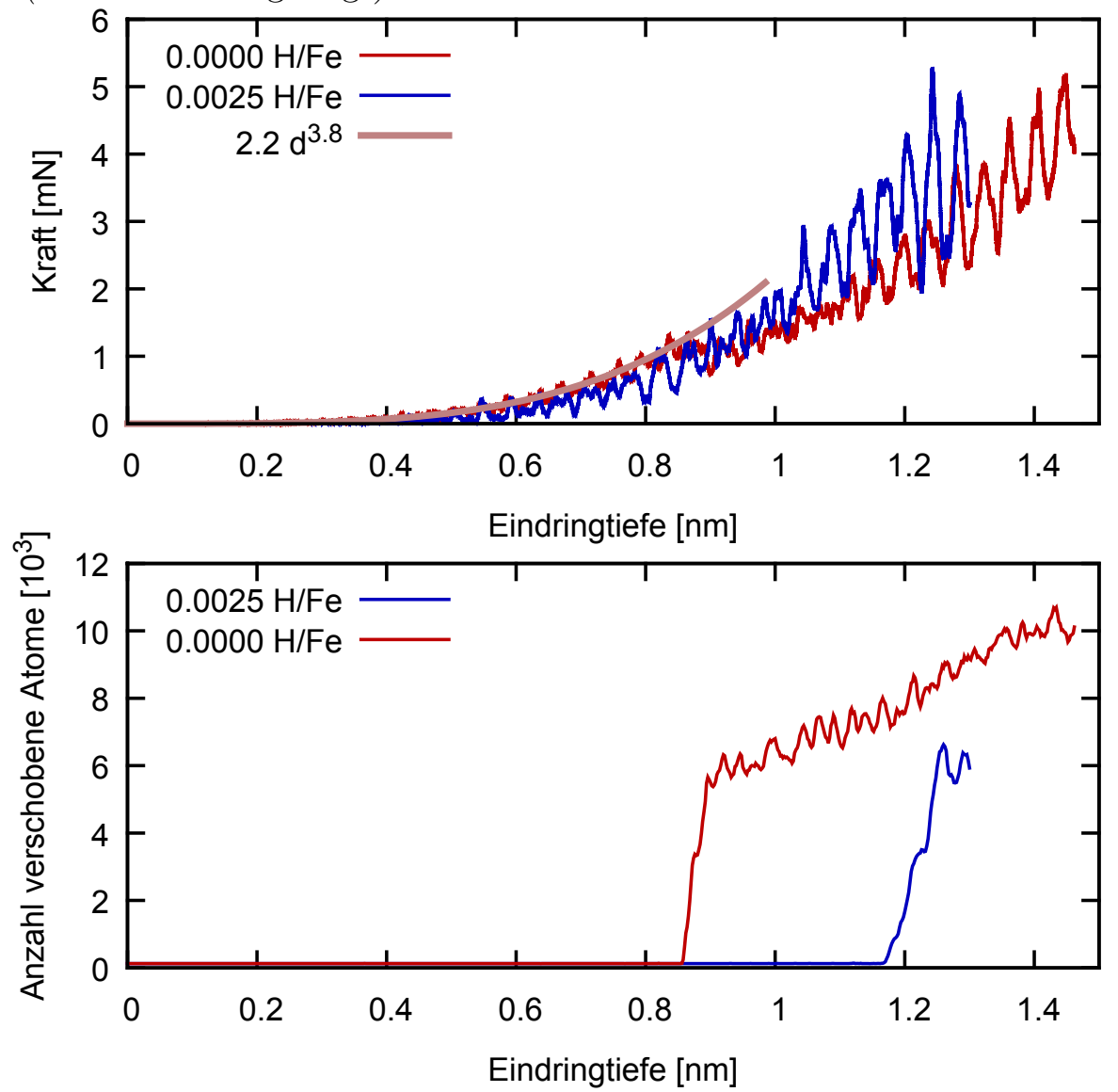

Abb. 33 Kraft-Eindringkurve von Eisen (oben), von (001)-orientierten Oberflächen für wasserstofffreies Eisen und Eisen mit einer Wasserstoffkonzentration $0.0025 \mathrm{H} / \mathrm{Fe}$. Die rosa Kurve wurde an den Verlauf der wasserstofffreien Kurve im Bereich 0 bis 0,8 nm angepasst und soll den Pop-In Effekt sichtbarer machen. Die untere Abbildung zeigt die Anzahl der Atome, welche nicht dem idealen Gitter entsprechen, also Versetzungen und Leerstellen, gegen die Eindringtiefe des Indenters. 


\subsubsection{Versetzungsanordnung unter Einfluss von Wasserstoff im ETEM}

Untersuchungen von Versetzungsbewegungen im ETEM wurden sowohl an Palladium als auch an Vanadium durchgeführt. Für diese Versuche wurden Zugproben aus Blech verwendet, in deren Mitte ein Loch geätzt wurde. Dadurch ist die Probe an den Rändern des Lochs durchlässig für Elektronen. Eine genaue Beschreibung der Probenpräparation befindet sich in Kapitel 5.1.3.

Diese Proben wurden solange gezogen, bis sich Versetzungen bewegt haben. Danach wurde gewartet bis diese Bewegung abgeschlossen war. Die Probe befindet sich nun unter Zugspannung und die Spannung im beobachtete Bereich ist nahe zur Fließgrenze.

Der Wasserstoffgasdruck wurde in diesen Experimenten schrittweise im Bereich von 0 bis 6 mbar erhöht und währenddessen wurde die beobachtete Stelle im Abstand von 0,2 s abgebildet.

\section{In-situ ETEM Zugversuche an Palladium}

Um die Auswirkung von Spannungen auf das Verhalten von Palladium zu untersuchen, muss vor dem Zugversuch die Stelle in der Probe gefunden werden, an der sich die Spannungen konzentrieren. In Abbildung 34 ist ein Übersichtsbild einer solchen Region zu sehen, in der im weiteren Verlauf des Versuchs die Beobachtungen durchgeführt wurden.

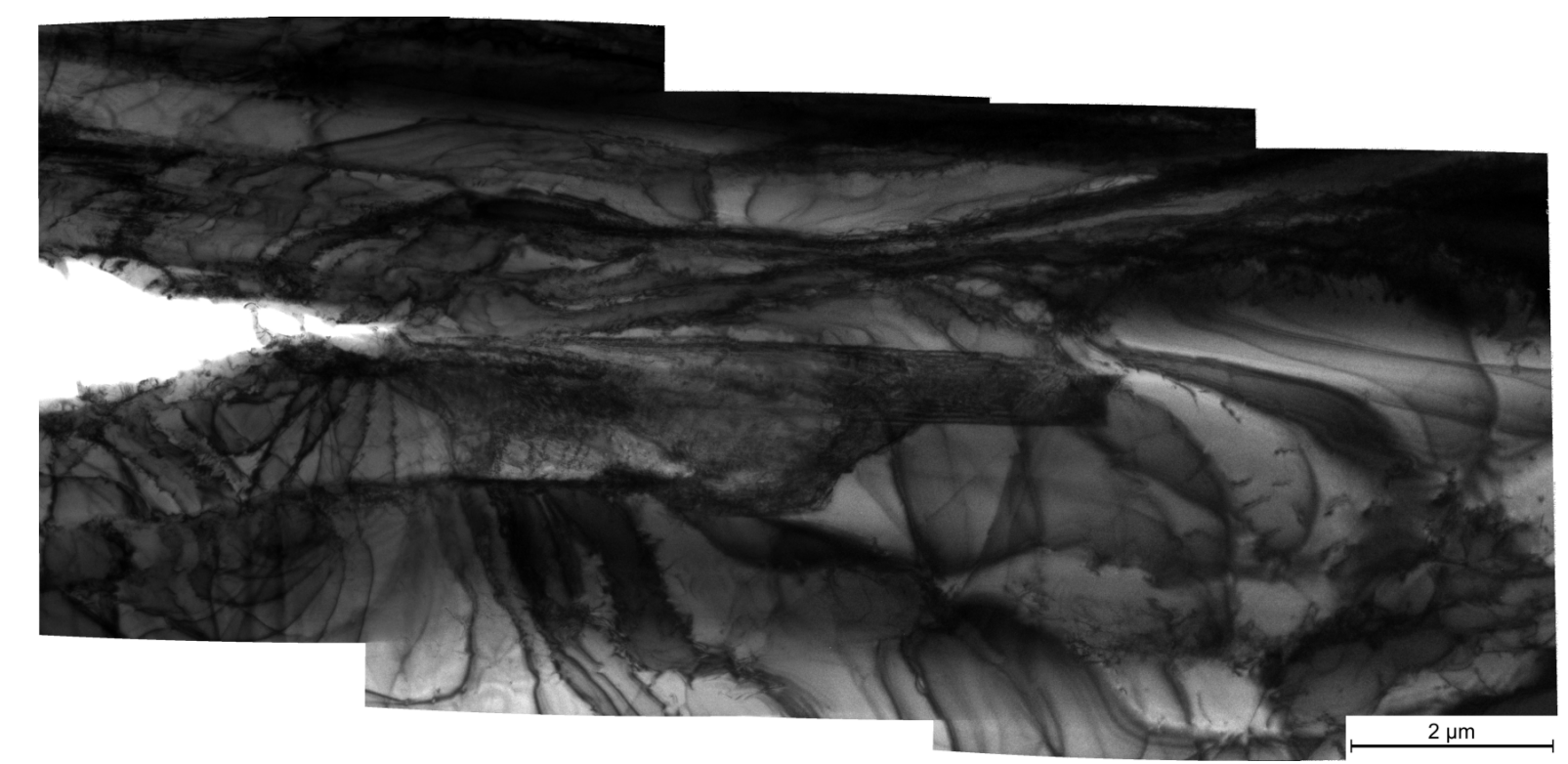

Abb. 34 Übersichtsbild der Umgebung um den untersuchten Probenbereich. An der linken Seite ist die Öffnung eines Risses zu erkennen, welcher im Material durch einen Stapelfehler (eckig erscheinende Struktur in der Mitte) endet. Deutlich zu erkennen sind Biegekonturen, die sich über die gesamte Probe verteilen. An den Stellen, an denen auch Versetzungen liegen sind die Biegekonturen unstetig, siehe z. B. unten in der Mitte. Einzelne Versetzungen sind im rechten Viertel in Form von kurzen Linien erkennbar. Die im weiteren Verlauf des Versuchs angelegte Zugbelastung erfolgte senkrecht zum Riss.

Für die einzelnen Beobachtungen wurde ein Bereich am Ende des zum Riss gehörenden Stapelfehlers gewählt. Die Bilderserie in Abbildung 35 zeigt die Vorgänge während des Ziehens. Dabei zeigt sich, dass sich der Stapelfehler in einzelnen Sprüngen vergrößert. Des Weiteren ist eine deutliche Bewegung der Biegekonturen zu erkennen, die jedoch unabhängig von der Vergrößerung des Stapelfehlers ist und die gesamte Zeit während des Ziehens zu beobachten ist. 

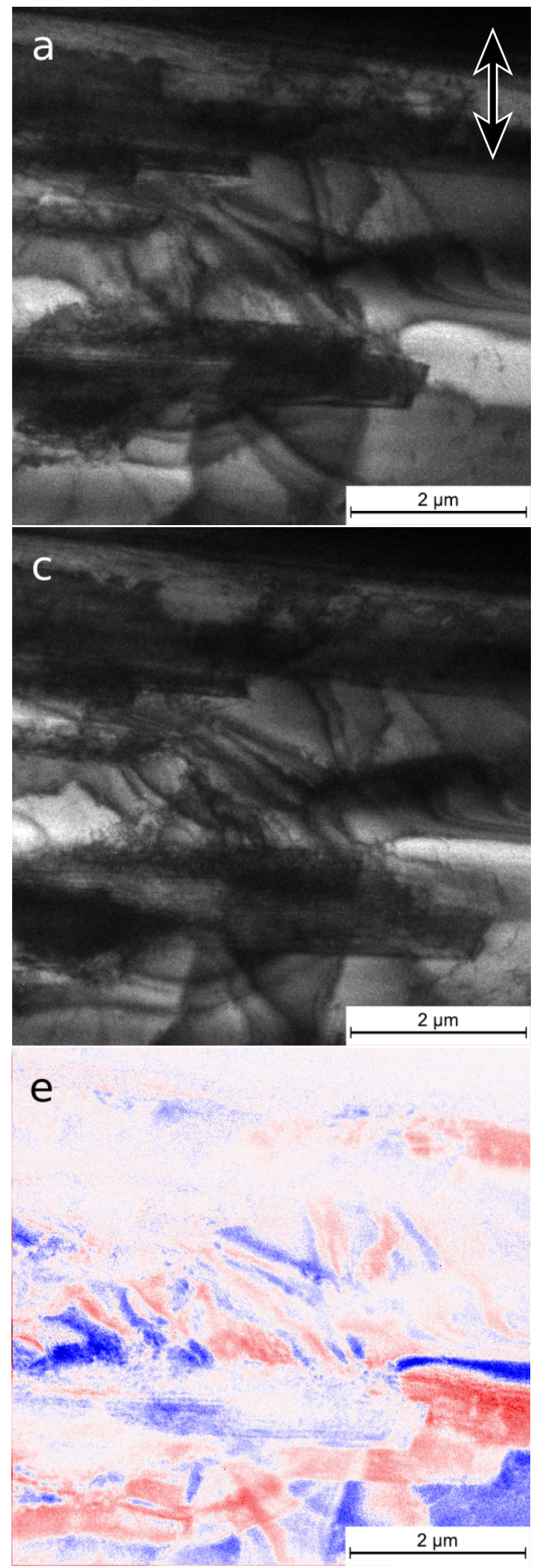
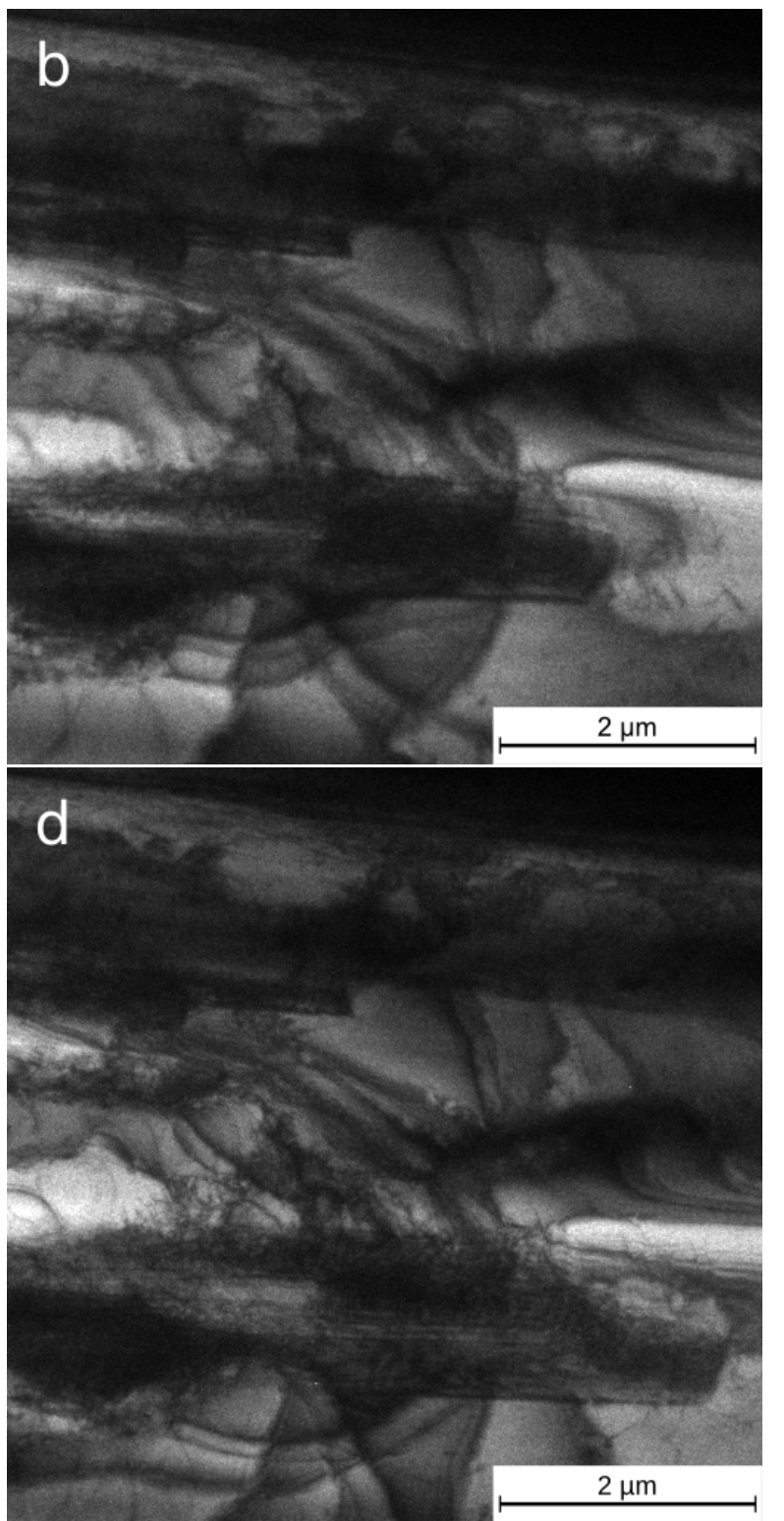

Abb. 35 Entwicklung einer Rissspitze in Palladium unter Zugbelastung. Der Pfeil in Abbildung a zeigt die Richtung der Zugbelastung an. Die Aufnahmen entstanden mit einem Abstand von 0,4 s. Zu erkennen ist, dass der Stapelfehler in der Bildmitte sich sprunghaft vergrößert. Um die Veränderungen in der Bilderserie zu verdeutlichen ist ein Differenzbild e zwischen dem Bild a und $\boldsymbol{d}$ erzeugt wurden. Rote Färbung zeigt dunkler gewordene Bereiche an und blaue Färbung zeigt heller gewordene Bereiche an. 

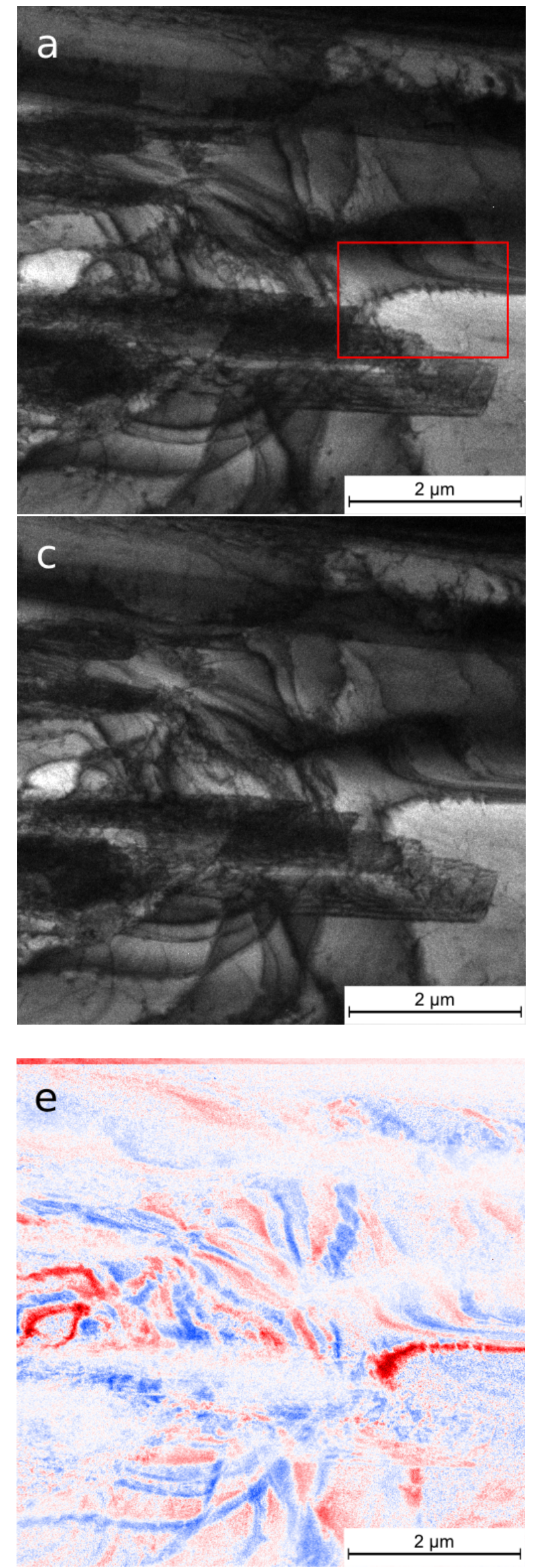
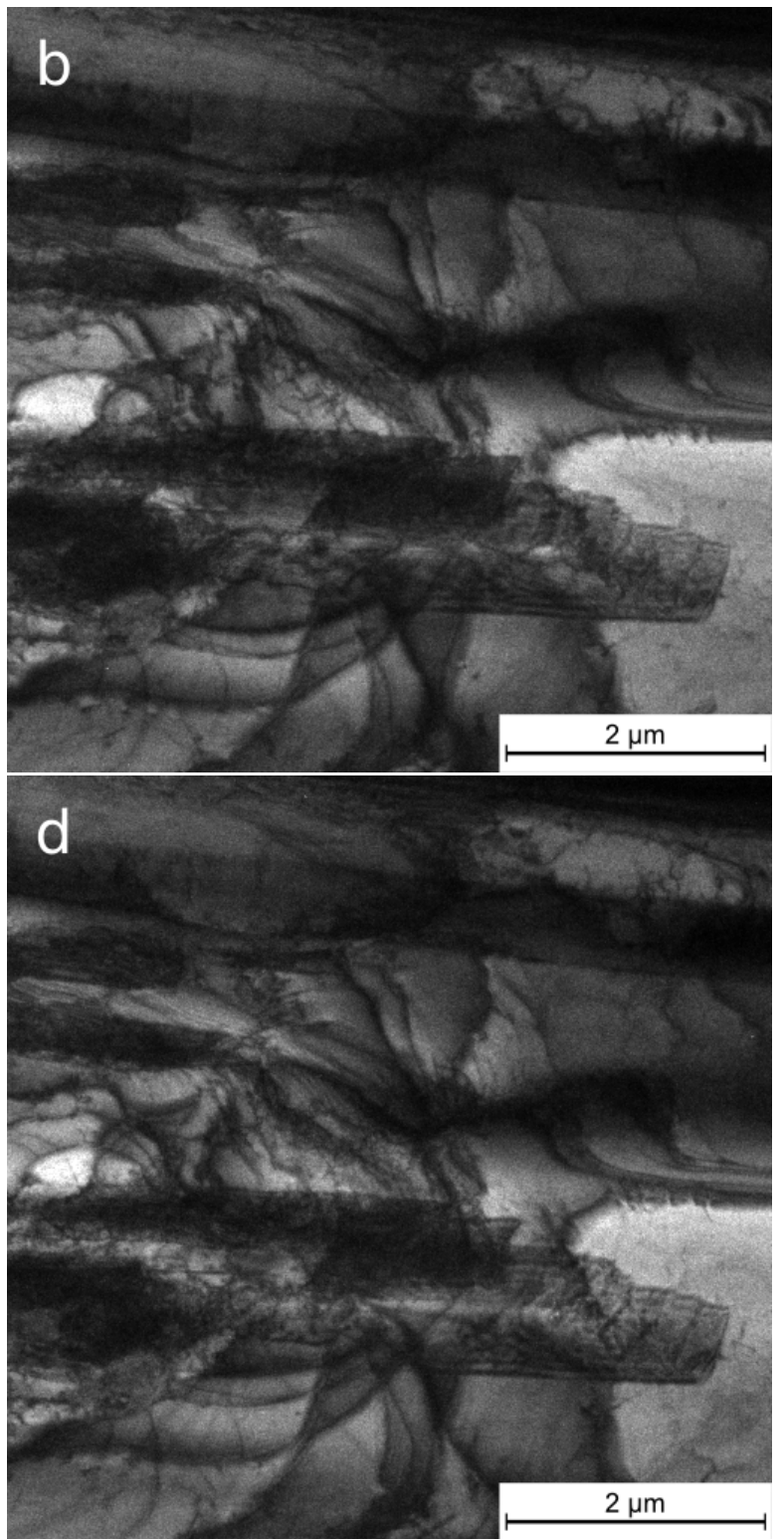

Abb. 36 Veränderung der Mikrostruktur bei Erhöhung des Drucks von 0 mbar auf 1 mbar. Die Aufnahmen entstanden im Abstand von 20 s. Der Druck wurde nach der Aufnahme des Bildes a erhöht und war zwischen dem Bild $\boldsymbol{b}$ und $\boldsymbol{c}$ abgeschlossen. Nach dem Bild $\boldsymbol{d}$ wurden keine Veränderungen mehr festgestellt. Die auffälligsten Veränderung sind die Bewegungen der Biegekonturen. Ein weiterer Effekt ist das Zusammenrücken von Versetzungen, siehe hier$\mathrm{zu}$ Bilderserie in der Abbildung 37, welche eine vergrößerte Darstellung des Bereichs im roten Kasten ist. Um die Veränderungen in der Bilderserie zu verdeutlichen ist ein Differenzbild e zwischen dem Bild a und $\boldsymbol{d}$ erzeugt wurden. Rote Färbung zeigt dunkler gewordene Bereiche an und blaue Färbung zeigt heller gewordene Bereiche an. 
In der Abbildungsserie in Abbildung 36 sind die Auswirkungen von Wasserstoffgas auf die Palladiumprobe dargestellt. Die Abbildungsserie zeigt die Ereignisse bei der ersten Gasdruckerhöhung von 0 auf 1 mbar. Bei dieser und auch bei jeder weiteren Druckerhöhung setzen sich sofort nach der Erhöhung die Biegekonturen in Bewegung. Nachdem der neue Druck erreicht ist, kommt die Bewegung erst über einen Zeitraum von mehreren Sekunden zu Stillstand. Sobald der Druck erneut erhöht wird, beginnt die Bewegung erneut.

Die Bewegung der Biegekonturen zeigt an, dass sich das Material verformt. Dadurch wird die gleichzeitige Beobachtung von Versetzungen erschwert, da die Darstellung dieser auf der exakten Ausrichtung der Probe zum Elektronenstrahl beruht. Jedoch gibt es in den Abbildungsserien 36 bzw. 37 Hinweise darauf, dass auch Versetzungen durch Wasserstoff beeinflusst werden. Diese Abbildungsserien zeigen Versetzungen, welche vor der Druckerhöhung in gleichmäßigen Abständen zueinander aufgestaut sind. Wird der Druck erhöht bewegt sich ein Teil der Versetzungen in eine Richtung und der andere Teil in die entgegengesetzte Richtung. Dabei erfolgt dies indem die Versetzungen ihren Abstand zueinander verkleinern.
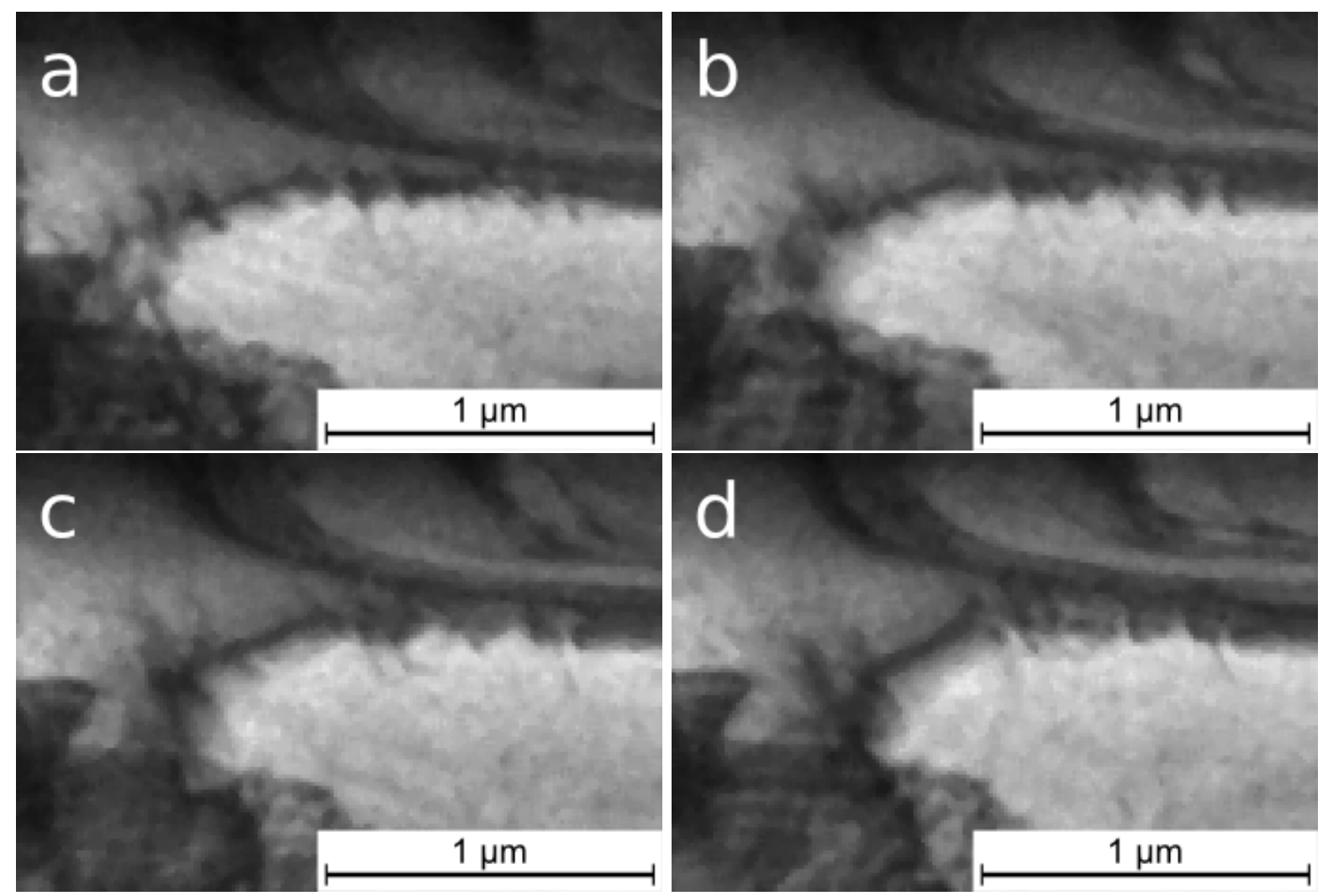

Abb. 37 Vergrößerter Ausschnitt aus der Bilderserie in Abbildung 36. Zu sehen sind mehrere aufgestaute Versetzungen. Bei Erhöhung des Druckes von 0 auf 1 mbar rücken die Versetzungen auseinander und bilden zwei Bereiche mit kleineren Abständen zwischen den Versetzungen. 


\section{In-situ ETEM Zugversuche an Vanadium}

Zur Untersuchung des Verhaltens der Mikrostruktur in Vanadium auf eine Wasserstoffatmosphäre wird analog zu den Palladiumversuchen vorgegangen. Der Bildvergleich in Abbildung 38 zeigt einen Ausschnitt der Ausläufer eines Risses. Merkmal dieser Ausläufer ist, dass diese zur Mitte hin immer dünner werden, jedoch nicht offen sind. Wird Wasserstoff hinzugegeben, wird beobachtet, dass einzelne Versetzungen springen.

Dies zeigt sich in Abbildung 38 durch Veränderungen im Zentrum der TEM-Bilder. Wird dieser Riss über mehrere Minuten beobachtet, kann eine Aufweitung erkannt werden.

Um diese Veränderungen deutlich zu machen wurde ein Differenzbild angefertigt, welches die absoluten Helligkeitsunterschiede darstellt. Hierbei bedeutet weiß kein Unterschied in der Helligkeit und schwarz bedeutet maximaler Unterschied. Dabei wurde das mittlere Bild in Abbildung 38 auf das linke Bild gelegt und so verschoben, dass der rechte Rand des Risses übereinstimmt. Dies dient dazu, den Drift der Probe auszugleichen. Anschließend wurde der absolute Helligkeitsunterschied berechnet.

Auf diesem Differenzbild ist eine von oben nach unten verlaufende Linie zu erkennen. Diese kommt aufgrund der Aufweitung des Risses zustande. Des Weiteren sind diffuse Kontraste links und rechts dieser Linie zu erkennen, welche auf die Bewegung von Versetzungen hindeuten.
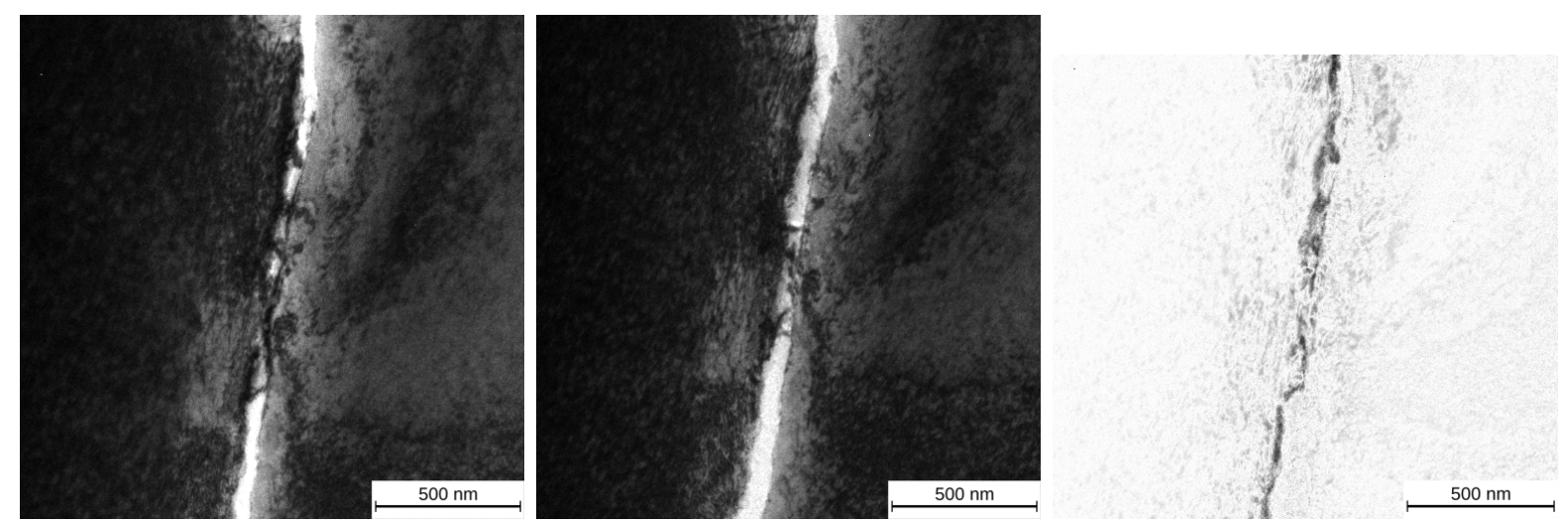

Abb. 38 Veränderung der Mikrostruktur in Vanadium bei Erhöhung des Drucks von 1 auf 3,5 mbar. Die Bilder wurden im Abstand von 7 Minuten aufgenommen. Das linke TEM-Bild zeigt den Zustand bei 1 mbar, das mittlere TEM-Bild den bei 3,5 mbar. Im rechten Bild ist die

Differenz der Helligkeit dargestellt; Weiß bedeutet kein Unterschied; Schwarz bedeutet maximaler Unterschied.

Dargestellt ist ein Ausschnitt der Ausläufer eines Risses im Material. Wird der Druck erhöht weitet sich der Riss. Dies ist erkennbar durch die senkrechte schwarze Linie im Differenzbild. Im

Zentrum der beiden TEM-Bilder sind Unterschiede in der Mikrostruktur erkennbar. Diese entwickeln sich während des Versuchs durch diskrete Sprünge, was ein Hinweis auf Versetzungsbewegung ist. 


\subsection{Einfluss von Wasserstoff auf die Versetzungsanordnung}

\subsection{1 Änderung der Verformungscharakteristik von Vanadium-Mikrosäulen}

In diesem Abschnitt wird das Verhalten von (001)-orientierten Vanadiumsäulen unter Druckbelastung beschrieben. Die Säulen wurden, wie in Kapitel 3.1.3 vorgestellt, präpariert und verformt. Die hier vorgestellten Säulen wurden wie in Tabelle 2 beschrieben verformt. Eine ausführliche Beschreibung dieser Experimente und deren Ergebnisse kann in der Veröffentlichung [44] nachvollzogen werden.

\begin{tabular}{c|c|c|c} 
Wasserstoffbeladung $[\mathrm{H} / \mathrm{V}]$ & Höhe $[\mu \mathrm{m}]$ & Dehnung maximal [-] & Dehnung Ende [-] \\
\hline \hline 0,0000 & 7,376 & 0,075 & 0,073 \\
0,0000 & 7,478 & 0,157 & 0,156 \\
\hline 0,0075 & 7,331 & 0,092 & 0,090 \\
0,0075 & 7,331 & 0,156 & 0,154 \\
\hline 0,0150 & 7,213 & 0,085 & 0,081 \\
0,0150 & 7,284 & 0,158 & 0,155 \\
\hline 0,0225 & 7,284 & 0,087 & 0,084 \\
0,0225 & 7,225 & 0,154 & 0,150 \\
\hline 0,0300 & 6,980 & 0,084 & 0,079 \\
0,0300 & 6,882 & 0,162 & 0,159
\end{tabular}

Tab. 2 Säulengröße vor der Verformung und Wasserstoffkonzentration bei Verformung der einzelnen Säulen. Des Weiteren ist die maximale Dehnung während der Verformung und die Dehnung nach Entlastung gezeigt.

In Abbildung 39 sind die Kraft-Dehnungskurven für verschiedene Wasserstoffkonzentrationen dargestellt. Es fällt auf, dass je mehr Wasserstoff in der Probe gelöst ist, desto mehr Druck wird benötigt, um die Säulen zu verformen. Im Anfangsbereich der Kurven sind bei niedrigen Konzentrationen ( 0 und $0.0075 \mathrm{H} / \mathrm{V}$ evtl. auch bei $0.0150 \mathrm{H} / \mathrm{V})$ Unregelmäßigkeiten zu erkennen, bei höheren Konzentrationen $(0.0225$ und $0.0300 \mathrm{H} / \mathrm{V})$ ist ein stetiger Verlauf zu sehen.

Die SEM-Aufnahmen der Säulen (Abbildung 40) zeigen bei niedrigen Konzentrationen (0 und $0.0075 \mathrm{H} / \mathrm{V}$ evtl. auch bei $0.0150 \mathrm{H} / \mathrm{V})$ Abgleitungen auf wenigen Gleitebenen, bei höheren Konzentrationen $(0.0225$ und $0.0300 \mathrm{H} / \mathrm{V})$ ist eine fassartige Verformung zu erkennen.
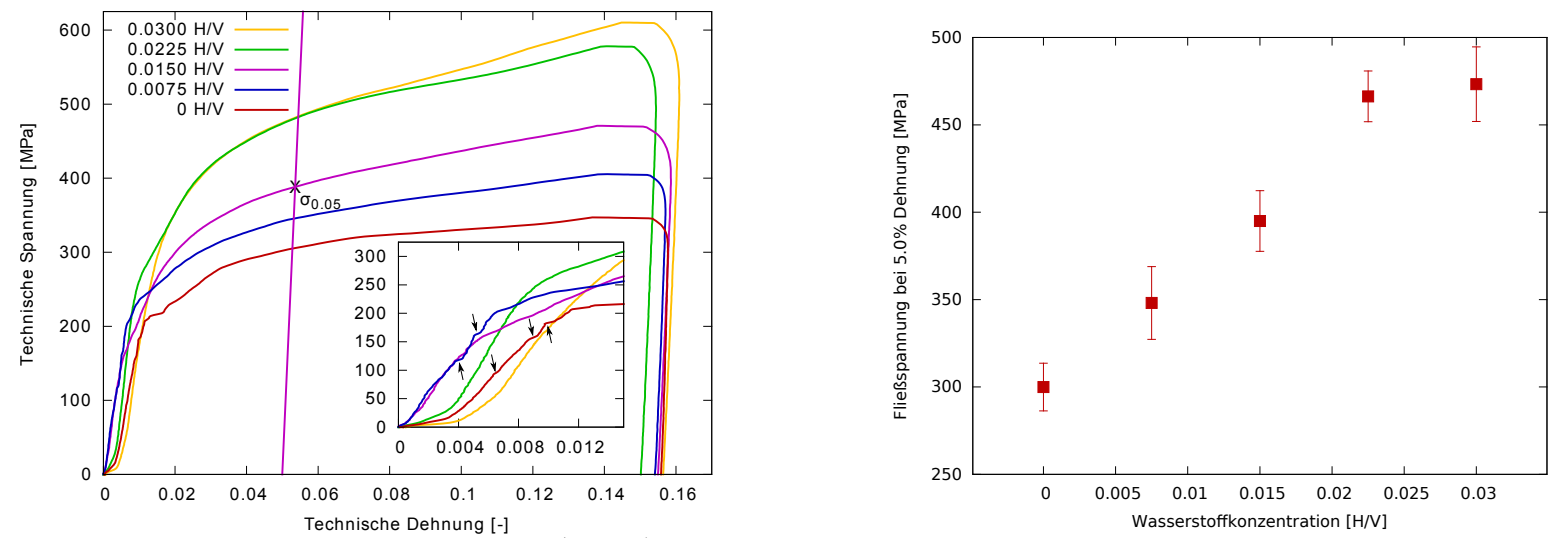

Abb. 39 Kraft-Dehnungskurven (links) der Säulen aus Abbildung 40 und die Fließspannung bei $5 \%$ Dehnung gegen die Wasserstoffkonzentration (rechts). Die Gerade in der linken Abbildung hat die gleiche Steigung, wie die Messwerte im elastischen Bereich der Säulen. Die Schnittpunkte mit den Messwerten ist die Fließspannung. Je mehr Wasserstoff bei der Verformung vorhanden ist desto mehr Kraft wird benötigt um die gleiche Verformung einzustellen.

Des Weiteren ist im Einschub in der linken Abbildung ein vergrößerter Ausschnitt des Anfangsbereichs zu sehen. Bei kleinen Konzentrationen treten Unregelmäßigkeiten im Anfangsbereich (Pfeile) auf, bei höheren Konzentrationen ist der Verlauf stetig. 
Bei genauer Betrachtung der SEM-Aufnahmen der verformten Säulen (Abbildung 40) fällt auf, dass bei den Wasserstoffkonzentrationen 0 und $0.0075 \mathrm{H} / \mathrm{V}$ die Säulen nur auf zwei verschiedenen

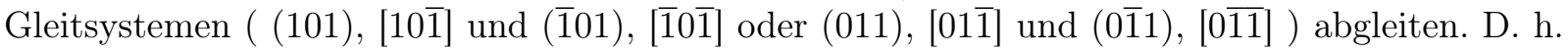
die Ebenennormalen liegen in einer Ebene. Ab einer Konzentration von $0.0150 \mathrm{H} / \mathrm{V}$ (Abbildung 40d) ist zu erkennen, dass die Verformung unter Verwendung aller vier Gleitebenenscharen erfolgt.
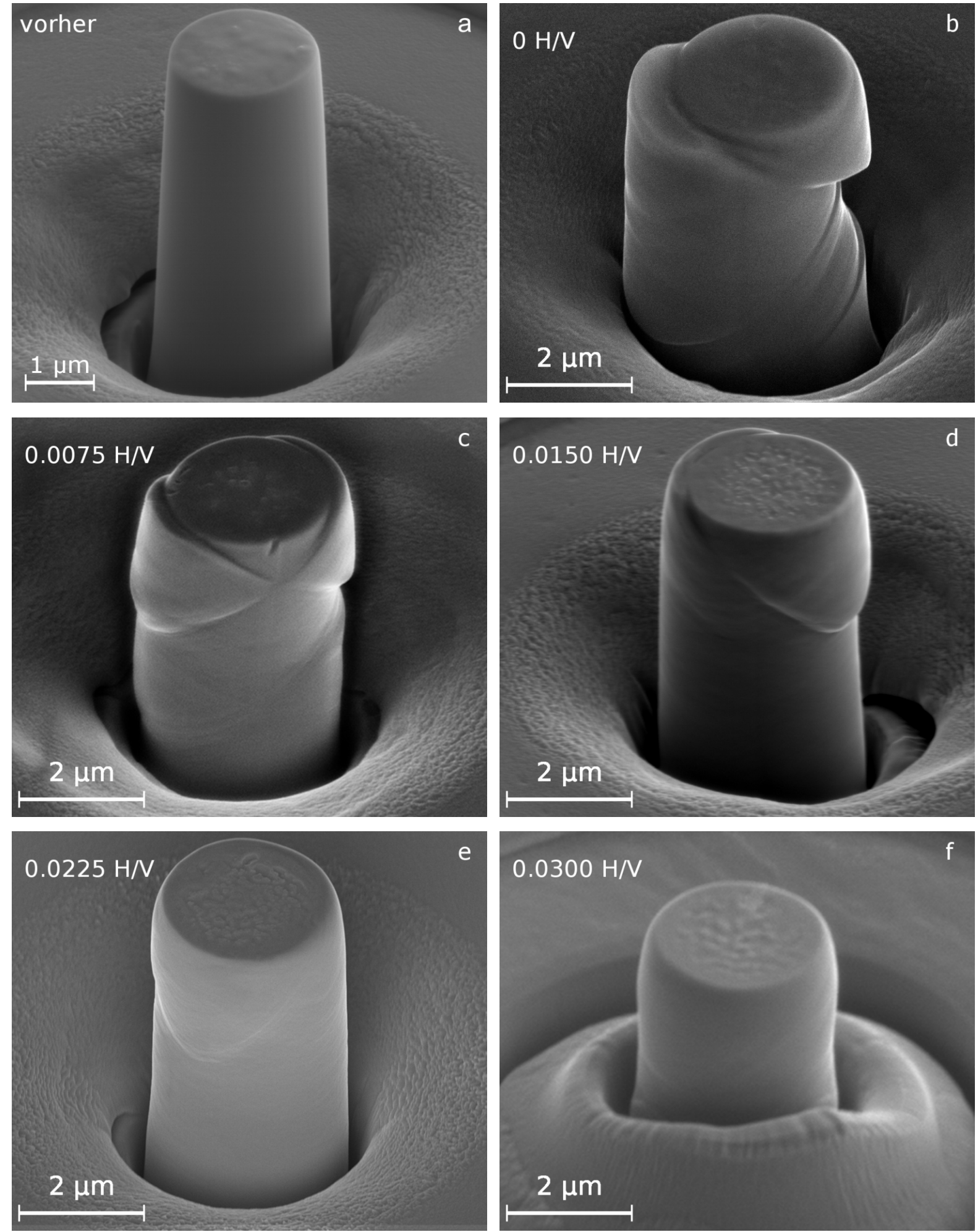

Abb. 40 REM Aufnahmen der Vanadiumsäulen. a Ausgangsform, $\boldsymbol{b}$-f Verformte Säulen, bei verschiedenen Wasserstoffkonzentrationen. Bei geringen Konzentrationen (b-d) erfolgt die Verformung auf einzelnen Gleitebenen, bei hohen Konzentrationen (e und $\mathbf{f}$ ) entsteht eine fassartige Verformung. Die REM Aufnahmen wurden unter einen $45^{\circ}$ Winkel zur Oberfläche aufgenommen. 
In Abbildung 41 ist eine Detailaufnahme einer Säule, welche mit einer Wasserstoffkonzentration von $0.03 \mathrm{H} / \mathrm{V}$ verformt wurde, zu sehen. Es ist zu erkennen, dass die Säule durch Abrutschen auf vielen Gleitebenen verformt wurde.

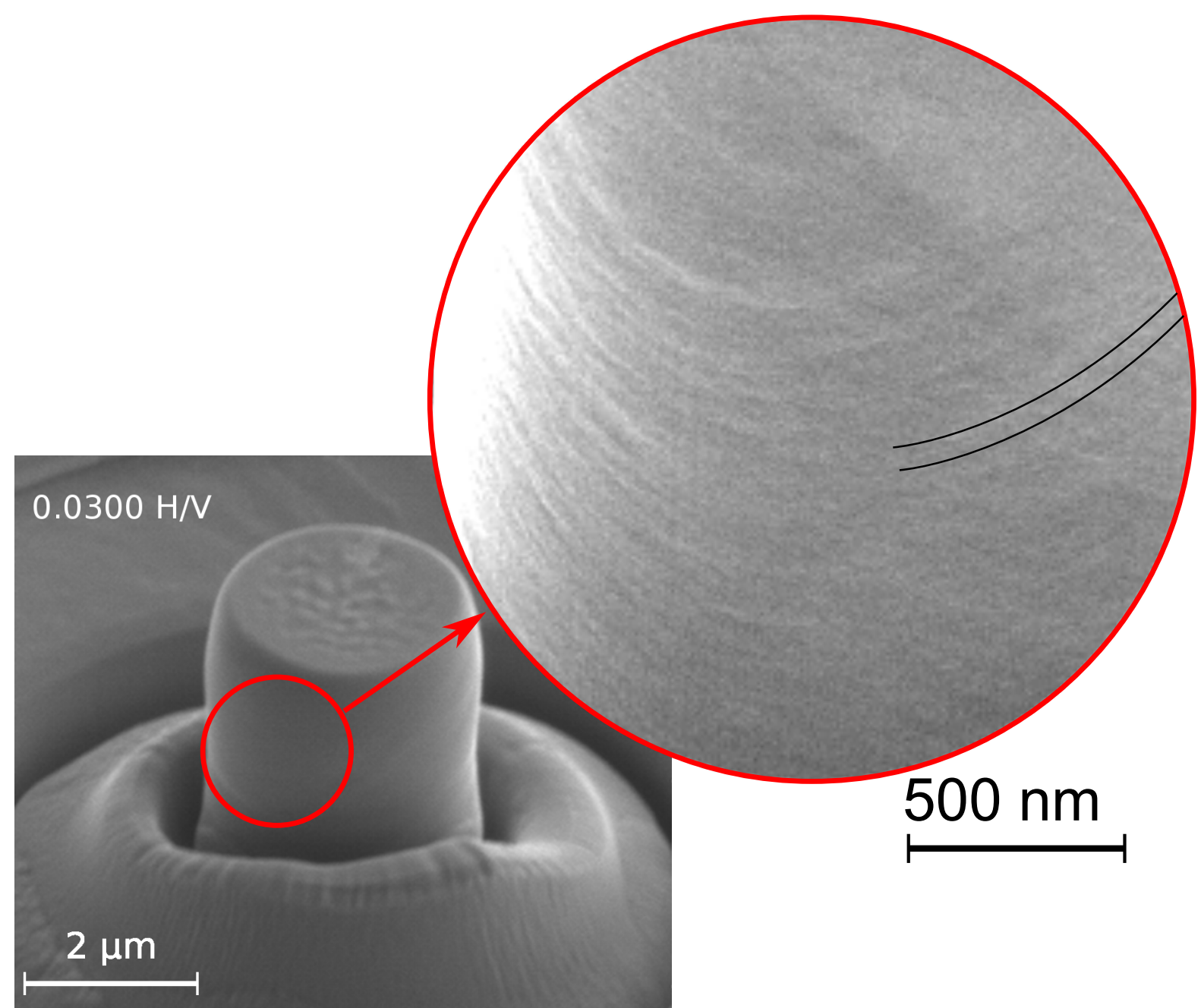

Abb. 41 Detailaufnahme einer verformten Vanadiumsäule, $0,03 \mathrm{H} / \mathrm{V} ; \epsilon=0,162$. Es sind viele Abgleitungen zu erkennen. Zur Veranschaulichung wurden zwei Gleitebenen durch Linien markiert. 


\subsubsection{Entwicklung der Versetzungsdichte von kaltgewalzten Palladium-Wasserstofflegierungen}

Das Walzen einer Probe ermöglicht es, eine genau definierte Verformung durchzuführen. Um den Einfluss von Wasserstoff auf die Versetzungsbildung bei Walzprozessen zu untersuchen, wurde ausgelagertes Palladiumblech mit verschiedenen Wasserstoffkonzentrationen beladen und anschließend kaltgewalzt (vgl. Kapitel 4.5). Eine ausführliche Beschreibung dieser Experimente und deren Ergebnisse kann in den Veröffentlichungen [40, 43] nachvollzogen werden.

\section{Mikrostruktur von kaltgewalzten Palladium-Wasserstofflegierungen}

Abbildung 42 zeigt die Versetzungsnetzwerke für verschiedene Wasserstoffkonzentrationen, welche sich beim Walzen gebildet haben, anhand von TEM-Aufnahmen.

Ohne Wasserstoff beim Walzen (Abbildung 42a) bilden sich große Versetzungszellen. Sobald Wasserstoff beim Walzen vorhanden ist $\left(c_{\mathrm{H}}=0.001 \mathrm{H} / \mathrm{Pd}\right.$, Abbildung $\left.42 \mathbf{b}\right)$, steigt die Anzahl der sichtbaren Versetzungen sprunghaft an. Für höhere Konzentrationen (Abbildung 42c und d) organisieren sich die Versetzungen wieder in Versetzungszellen. In Abbildung 42d sind die Zellwände so dicht, dass die inneren Bereiche der Versetzungszellen unterschiedliche Kontraste aufweisen. Dies deutet auf eine unterschiedliche Orientierung der Versetzungszellen hin, was als Bildung von Subkörnern interpretiert werden kann. Im Anhang (Kapitel 9.2.1) ist eine ähnliche Bilderserie mit höherer Vergrößerung zu finden (Abbildung 51a-d).
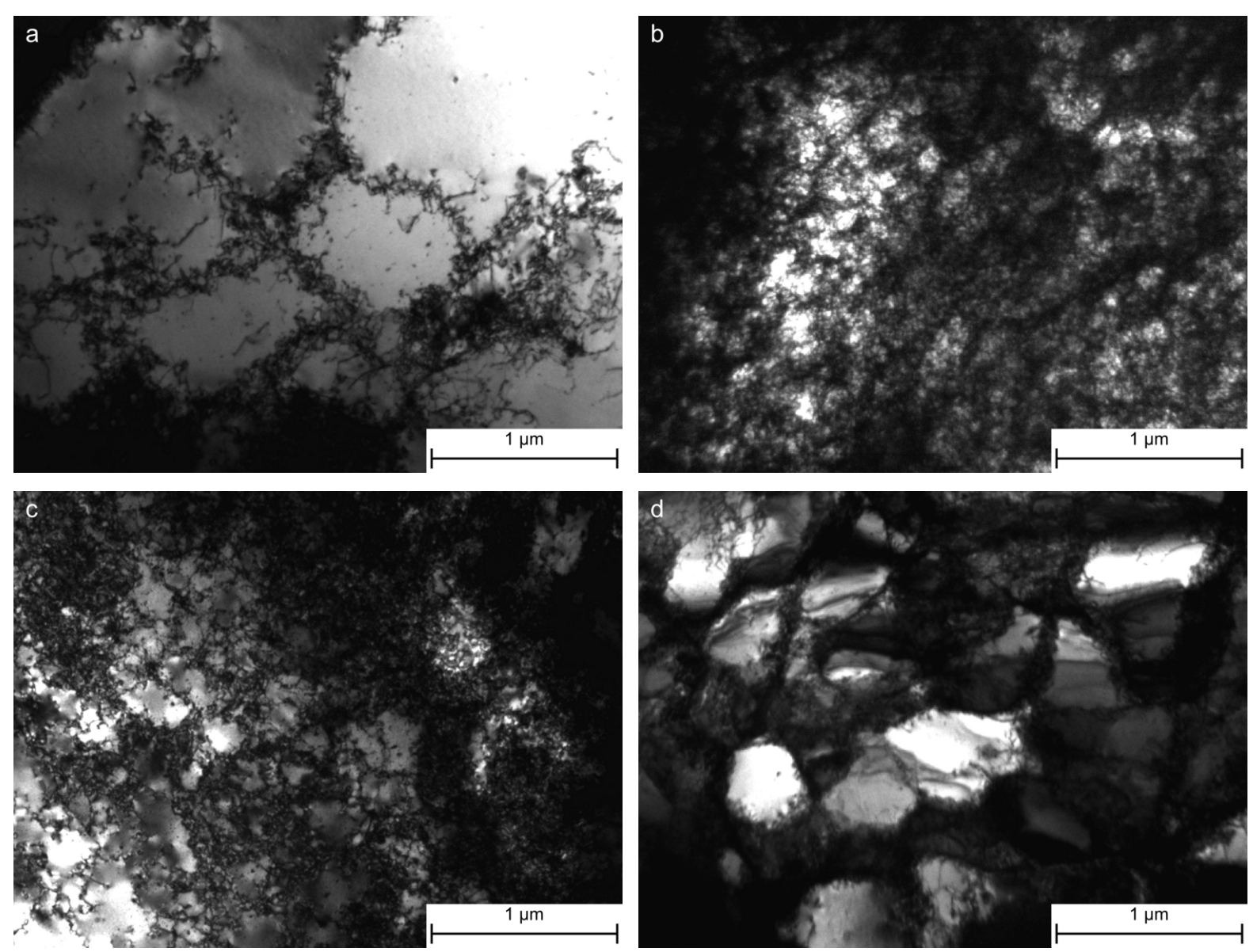

Abb. 42 TEM-Aufnahmen von kaltgewalzten Palladium-Wasserstofflegierungen [40], a zeigt eine Probe welche ohne Wasserstoff gewalzt wurde, die Abbildungen b-d zeigen TEM-Aufnahmen von kaltgewalzten Palladiumblechen, welche eine Wasserstoffkonzentration von $0.001 \mathrm{H} / \mathrm{Pd}$, $0.005 \mathrm{H} / \mathrm{Pd}$ bzw. $0.01 \mathrm{H} / \mathrm{Pd}$ bei der Verformung hatten. 


\subsubsection{Untersuchungen zur Defektbildung in Palladium-Wasserstofflegierungen bei extremer Verformung}

In diesem Abschnitt werden TEM Untersuchungen an Palladiumproben, welche von Dr. Y. Chen ${ }^{27}$ und Dr. D. Setman ${ }^{28}$ in der Arbeitsgruppe von Prof. Dr. M. Zehetbauer ${ }^{28}$ mittels HPT (vgl. Kapitel 4.6) verformt wurden, vorgestellt.

Ziel dieser Experimente war die Untersuchung des Effekts von Wasserstoff auf die Bildung von Leerstellen bei extremer Verformung unter verschiedenen Randbedingungen [48, 156, 157]. Im Rahmen dieser Arbeit stand die Untersuchung der Mikrostruktur im Vordergrund.

Die TEM Proben stammen aus der Mitte zwischen Zentrum der HPT-Probe und deren Rand und wurden konventionell präpariert. Dies bedeutet, dass die Probe senkrecht zur Druckrichtung gedünnt wurde. Die Präparation wurde analog zu den TEM-Proben bei kaltgewalztem Palladium durchgeführt und kann im Kapitel 3.1.4 nachvollzogen werden. In der Tabelle 3 sind die Versuchsparameter der hier vorgestellten Proben aufgelistet.

\begin{tabular}{|c|c|c|}
\hline$c_{H}[\mathrm{H} / \mathrm{Pd}]$ & $\begin{array}{l}\text { Verformungstemperatur } \\
\left(\mathrm{RT} T \approx 20^{\circ} \mathrm{C} ; \mathrm{CO}_{2} T \approx-60^{\circ} \mathrm{C}\right)\end{array}$ & $\begin{array}{l}\text { HPT Parameter } \\
\text { (Umdrehungen; Drehgeschwindigkeit } \\
{[\mathrm{RPM}] ; \text { Anpressdruck [GPa]) }}\end{array}$ \\
\hline 20,0025 & 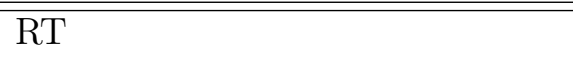 & $4 ; 0,2 ; 8$ \\
\hline 0,0025 & $\mathrm{CO}_{2}$ & $4 ; 0,2 ; 8$ \\
\hline 0,0075 & $\mathrm{RT}$ & $4 ; 0,2 ; 8$ \\
\hline 0,0075 & $\mathrm{CO}_{2}$ & $4 ; 0,2 ; 8$ \\
\hline 0,2000 & $\mathrm{RT}$ & $4 ; 0,2 ; 8$ \\
\hline 0,2000 & $\mathrm{CO}_{2}$ & $4 ; 0,2 ; 8$ \\
\hline 0,4500 & RT & $4 ; 0,2 ; 8$ \\
\hline 0,4500 & $\mathrm{CO}_{2}$ & $4 ; 0,2 ; 8$ \\
\hline
\end{tabular}

Tab. 3 Versuchsparameter der mittels HPT verformten Palladium Proben. Es wurden Proben mit Konzentrationen innerhalb der $\alpha$-Phase und im Zweiphasengebiet $\left(\alpha+\alpha^{\prime}\right)$ bei

Raumtemperatur und mit Trockeneiskühlung verformt.

Die Abbildungen 43 und 45 zeigen TEM Aufnahmen einer Palladiumprobe, welche mit 0,0025 H/Pd bei Raumtemperatur verformt wurde. $\mathrm{Zu}$ sehen ist, dass Körner mit einer mittleren Größe von $200 \mathrm{~nm}$ (vgl. Abbildung 47) entstanden sind. Die Kornstruktur ist klar zu erkennen, da im inneren der Körner wenige Versetzungen vorliegen. Des Weiteren gibt es Kleinwinkelkorngrenzen, welche dadurch erkennbar sind, dass hier Versetzungen aufgestaut sind.

Ein völlig gegensätzliches Bild zeigt sich bei der Probe, welche bei der Verformung eine Wasserstoffkonzentration von $0,45 \mathrm{H} / \mathrm{Pd}$ aufwies. In den TEM-Aufnahmen dieser Probe (Abbildungen 44 und 46) sind kaum noch einzelne Körner sichtbar, zu dem ist die sichtbare Versetzungsdichte erheblich höher. Im Übersichtsbild 44 ist des Weiteren zu erkennen, das nur wenige jedoch ungewöhnlich geformte Körner vorliegen, welche aufgrund der hohen Versetzungsdichte leichte Orientierungsvariationen aufweisen, was an Biegekonturen erkennbar ist. Diese Beobachtungen spiegeln sich wider in den Beugungsbildern. In Abbildung 44 sind Unterbrechungen in den Ringen zu erkennen, dies weist auf eine Texturierung hin.

Aufnahmen der anderen Proben befinden sich im Anhang im Kapitel 9.2.2.

\footnotetext{
${ }^{27}$ State Key Lab of Solidification Processing, Northwestern Polytechnical University, Xián,

People's Republic of China

${ }^{28}$ Groups Physics of Nanostructured/Functional Materials, Universität Wien
} 

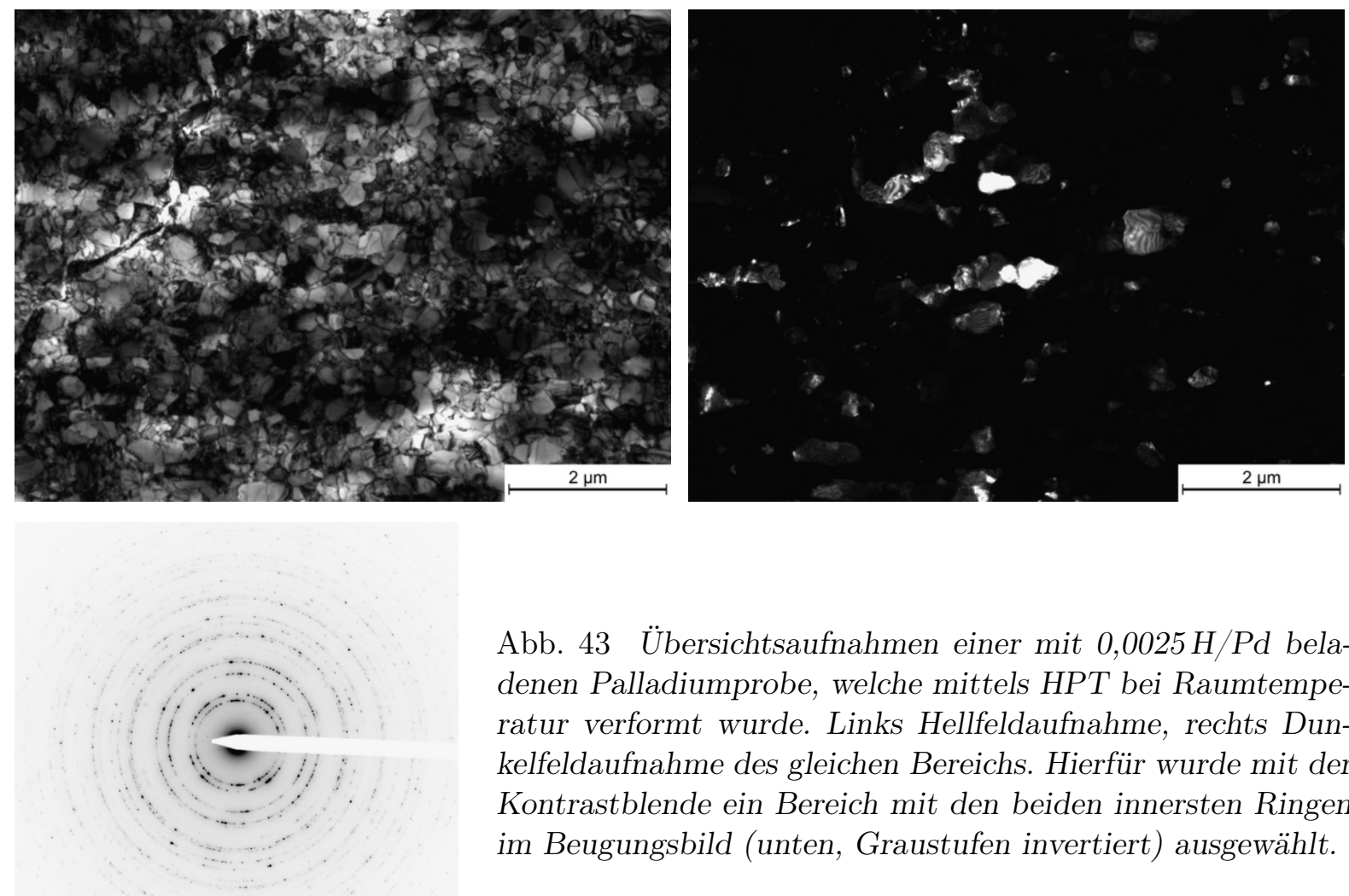

Abb. 43 Übersichtsaufnahmen einer mit 0,0025 H/Pd beladenen Palladiumprobe, welche mittels HPT bei Raumtemperatur verformt wurde. Links Hellfeldaufnahme, rechts Dunkelfeldaufnahme des gleichen Bereichs. Hierfür wurde mit der Kontrastblende ein Bereich mit den beiden innersten Ringen im Beugungsbild (unten, Graustufen invertiert) ausgewählt.
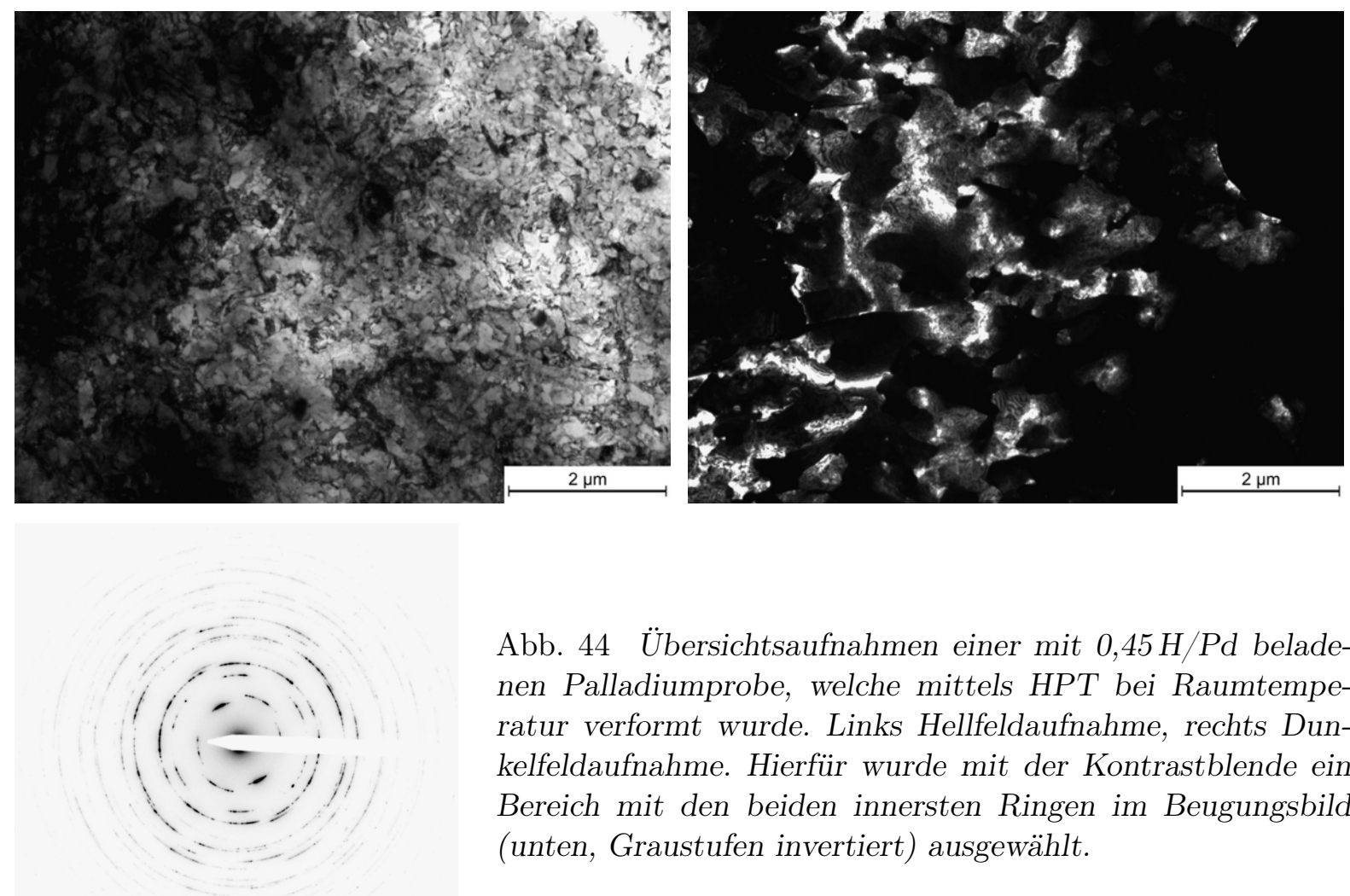

Abb. 44 Übersichtsaufnahmen einer mit 0,45 H/Pd beladenen Palladiumprobe, welche mittels HPT bei Raumtemperatur verformt wurde. Links Hellfeldaufnahme, rechts Dunkelfeldaufnahme. Hierfür wurde mit der Kontrastblende ein Bereich mit den beiden innersten Ringen im Beugungsbild (unten, Graustufen invertiert) ausgewählt. 

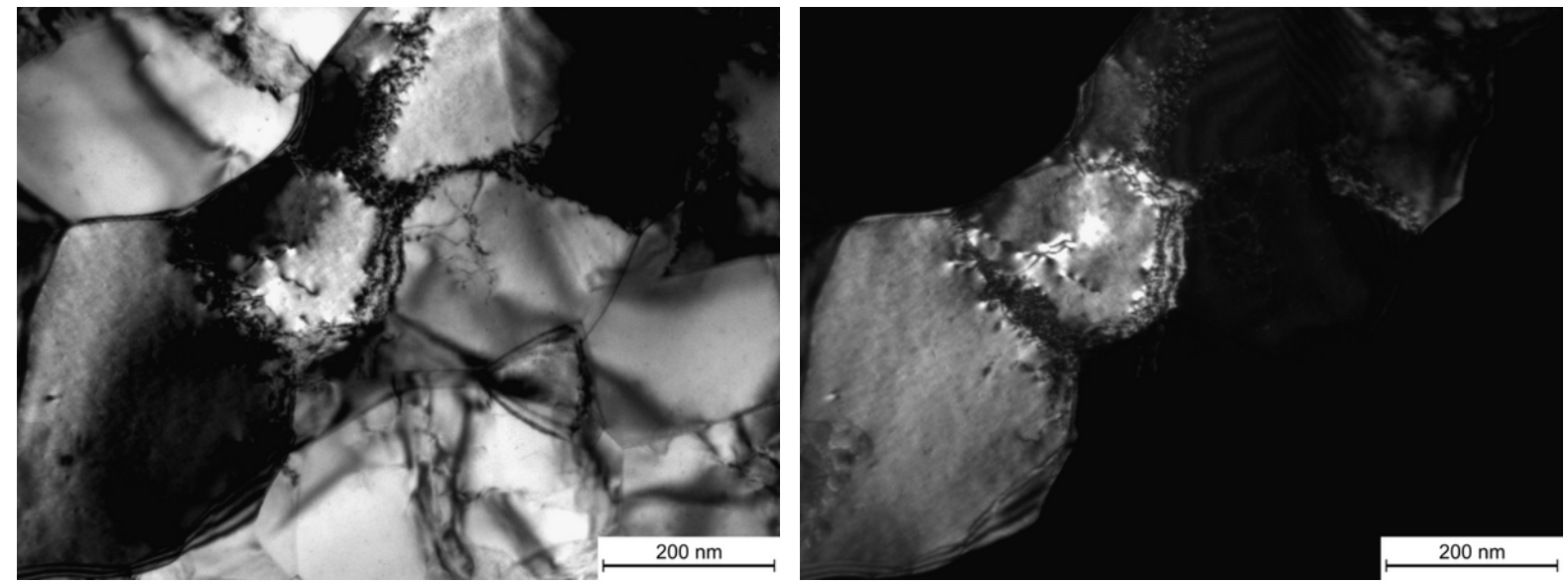

Abb. 45 Detailaufnahmen einer mit 0,0025 H/Pd beladenen Palladiumprobe, welche mittels HPT bei Raumtemperatur verformt wurde. Links Hellfeldaufnahme, rechts Dunkelfeldaufnahme des gleichen Bereichs.
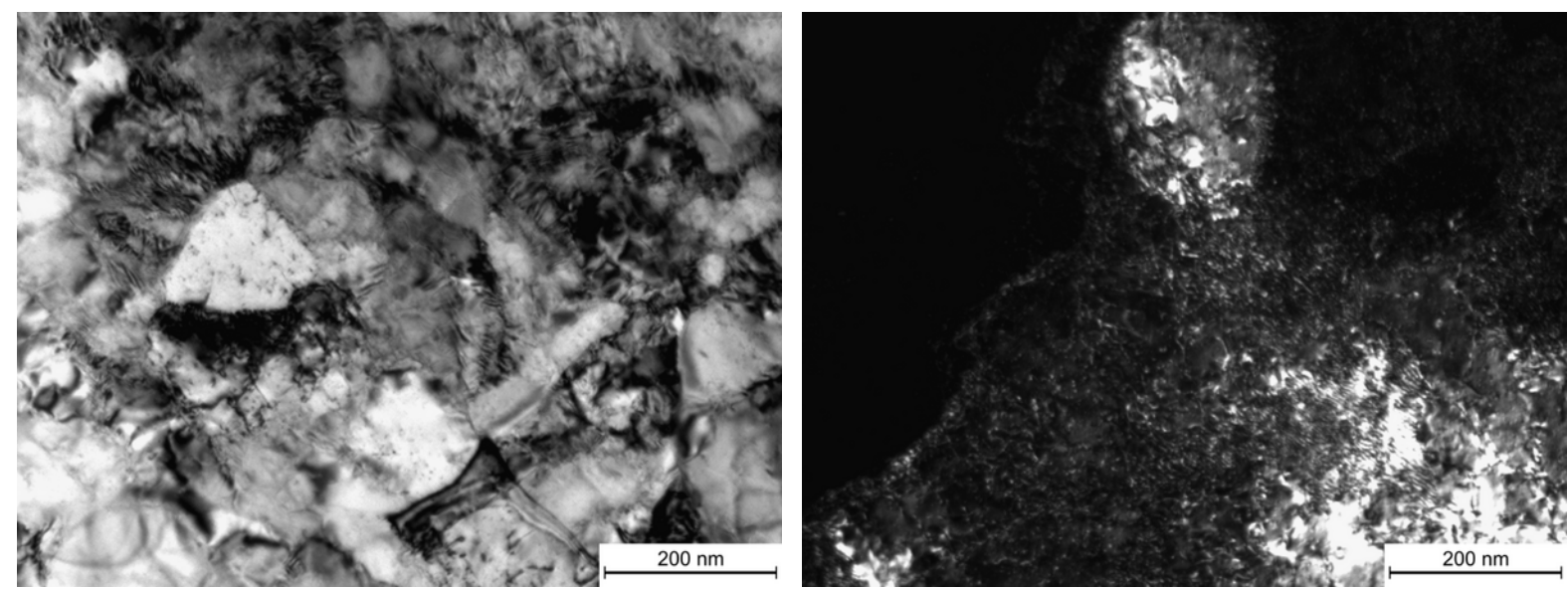

Abb. 46 Detailaufnahmen einer mit 0,45 H/Pd beladenen Palladiumprobe, welche mittels HPT bei Raumtemperatur verformt wurde. Links Hellfeldaufnahme, rechts Dunkelfeldaufnahme.

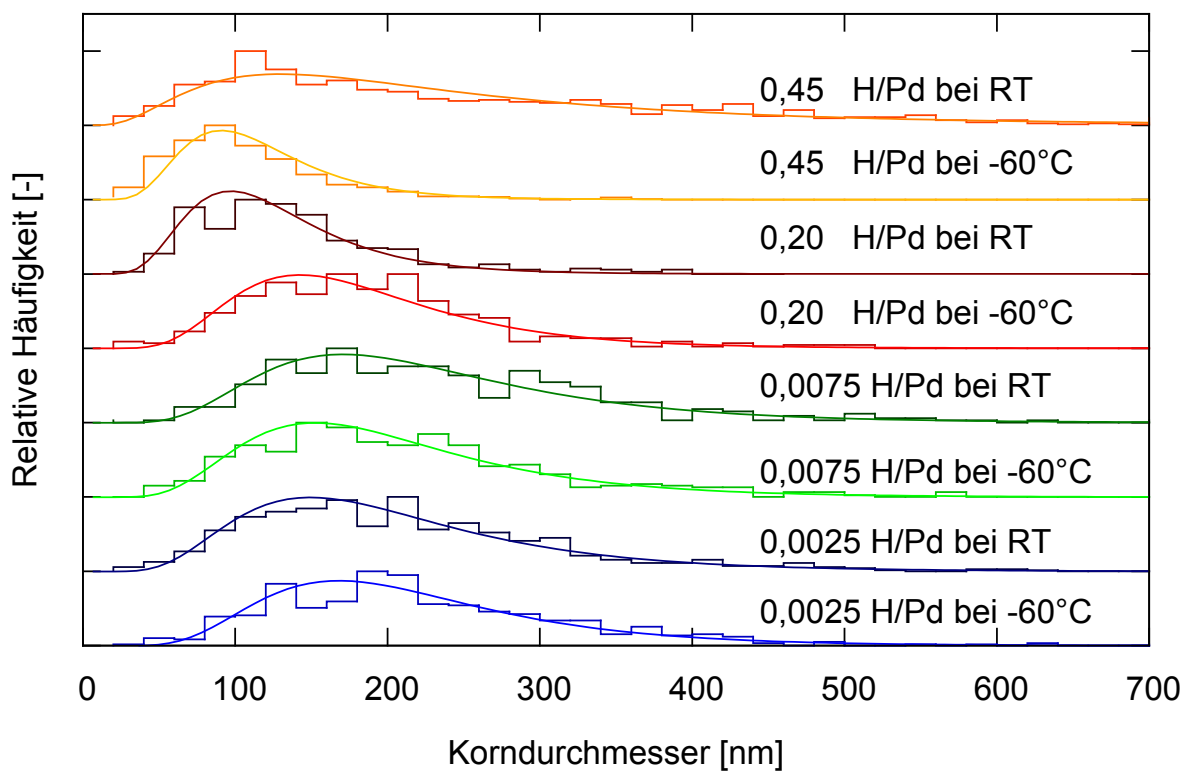

Abb. 47 Verteilung der Korngrößen von mittels HPT verformten Palladium-Wasserstofflegierungen. 


\section{Diskussion}

Wasserstoff hat, wie in der Einführung geschildert, in vielen Fällen einen negativen Einfluss auf mechanische Eigenschaften von Metallen. Dieses Phänomen wird unter dem Begriff Wasserstoffversprödung zusammengefasst [1]. Die Wasserstoffversprödung entzieht sich bisher einer einheitlichen theoretischen Beschreibung, da viele hochkomplexe Vorgänge daran beteiligt sind [25, 77, 88, 158]. Im Rahmen dieser Arbeit wurden daher verschiedene Experimente durchgeführt, um die zugrundeliegenden mikrostrukturellen Vorgänge besser zu verstehen. Für die Untersuchungen wurden Modellsysteme verwendet, die es erlauben, einzelne Vorgänge der Wasserstoffversprödung unabhängig voneinander zu untersuchen. Dazu wurde in verschiedenen Metallproben Wasserstoff gelöst und anschließend auf unterschiedlichste Arten verformt. Dabei lag der Schwerpunkt auf der Untersuchung der Entstehung von Versetzungen unter Wasserstoffeinfluss.

Bei der Diskussion der Ergebnisse dieser Arbeit werden zunächst die beobachteten Effekte von Wasserstoff auf einzelne Versetzungen behandelt. Danach werden die gewonnenen Erkenntnisse zur Entstehung und Interaktion von Versetzungen beschrieben. Daran anschließend werden die Auswirkungen von Wasserstoff auf die Linienenergie von Versetzungen quantifiziert und erörtert. Abschließend werden die Ergebnisse zusammenfassend diskutiert und der Übergang zu makroskopischen Vorgängen vorgenommen.

\subsection{Einfluss von Wasserstoff auf einzelne Versetzungen}

Im Rahmen dieser Arbeit wurden zwei experimentelle Methoden verwendet, welche es erlauben den Einfluss von Wasserstoff auf einzelne Versetzungen direkt zu untersuchen. Bei diesen Methoden handelt es sich um die Nanoindentation und um die Beobachtung einzelner Versetzungen in einem Environmental Transmissionselektronenmikroskop (ETEM).

Die Nanoindentation erlaubt es hochpräzise Kraft-Eindringkurven bei der Indentierung eines Materials aufzunehmen. Damit lässt sich der Übergang von elastischem zu plastischem Verformungsverhalten studieren. Dieser Übergang wird bei einkristallinem Material durch die Bildung von Kristalldefekten begleitet [159].

Die direkte Beobachtung im ETEM erlaubt es die Reaktionen von Versetzungen auf eine Wasserstoffgasatmosphäre zu untersuchen. Wird Wasserstoff in Form von Gas angeboten, nimmt die Probe einen Teil auf. Dieser Wasserstoff kann mit den Versetzungen interagieren, was zur Bewegung dieser führen kann.

\section{Generelles Verhalten von einkristallinen Metallen bei Nanoindentation}

Die im Rahmen dieser Arbeit durchgeführten Nanoindentationsversuche an Vanadium- und Palladium-Wasserstofflegierungen (vgl. Kapitel 5.1.1) zeigen, das für diese Art von Nanoindentationsversuchen typische Verhalten [160].

Die Kraft-Eindringkurven der Versuche am Vanadium zeigen zwei waagerechte Abschnitte. Diese Abschnite sind charakteristisch für Pop-In Effekte. Dabei ist der erste unabhängig vom Wasserstoffgehalt. Die Ursache für den ersten Pop-In konnte im Rahmen dieser Arbeit nicht vollständig ergründet werden. Es wird angenommen, dass dieser Pop-In auf die Oxidschicht des Vanadiums zurückzuführen ist. Der weitere Verlauf der Kraft-Eindringkurven der Versuche am Vanadium und die Kraft-Eindringkurven der Versuche am Palladium weisen dabei drei Bereiche auf. Bei kleinen Eindringtiefen ergibt sich ein ideales elastisches Verhalten, welches sich durch einen Hertzschen Kontakt beschreiben lässt $[145,148]$, d. h. die aufzuwendende Kraft $F$ hängt mit der Eindringtiefe $d$ durch eine Funktion der Form $F=a \cdot d^{b}$ zusammen ( $a$ und $b$ freie Parameter).

Wird eine kritische Kraft überschritten, sinkt der Indenter mehrere Nanometer ins Material, ohne dass die Kraft erhöht wird. Dieses Phänomen wird Pop-In genannt. Der Pop-In wird in der Literatur [109, 144-147] damit erklärt, dass bei dieser kritischen Kraft neue Defekte entstehen. 
Wird bspw. ein Versetzungsring gebildet, benötigt dies eine hohe Energie. Ist der Versetzungsring jedoch gebildet, benötigt die Vergrößerung des Durchmessers kaum noch Energie, solange die Versetzung nicht auf ein Hindernis trifft. Somit ist eine weitreichende Verformung des Materials ohne hohen Energieaufwand möglich.

Daran anschließend steigt die Kraft-Eindringkurve. Dies bedeutet, dass neue Versetzungen gebildet werden oder vorhandene Versetzungen Hindernisse überwinden müssen. Da bei der Nukleation weiterer Versetzungen schon andere Versetzungen vorhanden sind, kommt es auch zur Interaktion zwischen einzelnen Versetzungen. Dieses Verhalten von Versetzungen ist typisch für plastische Verformung [159]. Dies bedeutet, dass der Pop-In Effekt den Übergang vom elastischen zu plastischem Verhalten markiert.

\section{Vergleich mit Molekulardynamiksimulationen}

Um die bei der Nanoindentation auftretenden Prozesse zu veranschaulichen, wurden Molekulardynamiksimulationen (MD) von Nanoindentationsversuchen durchgeführt, vgl. Kapitel 5.1.2. In den Abbildungsserien 27 bzw. 30 wird die Entstehung von Versetzungen in Palladium bzw. Eisen bei Nanoindentierung dargestellt. Im Folgenden werden die bei den Simulationen auftretenden Vorgänge, welche bei den Simulationen der reinen Metalle auftreten, behandelt.

\section{MD-Simulation von Palladium}

Die Simulation der Nanoindentation von kfz-Palladium zeigt das aus Experimenten gefolgerte Verhalten [160]. Zunächst zeigt sich eine elastische Antwort in der Kraft-Eindringkurve (Abbildung 29), welches aufgrund der mit dem Hertzschen Kontaktmodell beschreibbaren Kurve gefolgert werden kann. Darauf folgt ein waagerechter Abschnitt. Daran anschließend steigt die Kraft mit zunehmender Eindringtiefe des Indenters weiter an.

$\mathrm{Zu}$ den einzelnen Abschnitten in den Kraft-Eindringkurven werden die im Folgenden beschriebenen Defektmechanismen, welche in den Darstellungen der Abbildungsserie 27 zu erkennen sind, zugeordnet: Im elastischen Bereich entstehen zunächst keine Versetzungen. Wird die Eindringtiefe weiter erhöht bilden sich zum Ende des elastischen Bereiches hin erste Stapelfehler aus. Dies wird daran sichtbar, dass sich Scheiben im Gitter ausbilden, welche eine lokale hexagonal dichtest gepackte (hdp) Struktur aufweisen. Die Bildung von Stapelfehlern stellt nicht den Beginn des Pop-Ins dar, jedoch sind erste Abweichungen vom idealen Verhalten nach dem Hertzschen Kontaktmodell zu erkennen.

Bei weiterer Verformung bilden sich aus den Stapelfehlern halbringförmige Strukturen, deren Enden sich an der Oberfläche befinden. Die Atome innerhalb der Stapelfehler ordnen sich also wieder so an, dass im Inneren der Stapelfehler wieder eine lokale kfz Struktur vorliegt. Wie in Kapitel 2.1 beschrieben handelt es sich bei diesem Vorgang um die Bildung einer vollständigen Versetzung. Die Bildung dieser vollständigen Versetzungen fällt mit dem Beginn des waagerechten Abschnittes in den Kraft-Eindringkurven, dem Pop-In, zusammen. Der Durchmesser der Versetzungsringe nimmt mit zunehmender Eindringtiefe zu, ohne dass deutlich mehr Kraft aufgewendet werden muss. Dieses Verhalten wird durch den gleichzeitigen rapiden Anstieg der Anzahl der aus der idealen krz Position verschobenen Atome sichtbar (vgl. Abbildung 29). Im Rahmen dieser Arbeit wird aus diesem Verhalten gefolgert, dass erst die Bildung von vollständigen und mobilen Versetzungen den Pop-In Effekt ermöglichen.

Da diese ersten Versetzungen sich von der Position des Indenters wegbewegen, können diese die vom Nanoindenter lokal aufgebrachte Verspannung nicht für beliebige Eindringtiefen ausgleichen. Bei weiterer Verformung müssen sich neue Versetzungen bilden. Dies geschieht wieder in Form von Stapelfehlern, welche sich nach und nach zu vollständigen Versetzungen umformen. Jedoch können sich diese sekundären Versetzungen nicht frei ausbreiten, da diese mit den bereits vorhandenen Versetzungen interagieren. Diese Interaktion fällt mit dem Ende des Pop-Ins zusammen. 


\section{MD-Simulation von Eisen}

Bei der Simulation der Nanoindentation von krz-Eisen folgt die Kraft-Eindringkurve nicht so gut dem experimentellen Verhalten, vgl. Kapitel 5.1.2. Der Grund hierfür sind wahrscheinlich unnatürliche Schwingungen der Oberfläche aufgrund von Ungenauigkeiten in der Simulation. Deswegen wurden verschiedene Potentiale zur Simulation des Eisen-Wasserstoffsystems verwendet. Zur Diskussion genereller materialphysikalischer Vorgänge im krz Gitter eignet sich das Potential von Kimizuka et. al. [153], da verschiedenste Versetzungsvorgänge erkennbar sind. Das Potential von Wen et. al. [136] eignet sich besser zur Untersuchung mechanischer Eigenschaften, da sich dieses Potential in vielen Arbeiten $[161,162]$ bewährt hat und daher die Eigenschaften von Eisen besser darstellt.

Für die Bildung von Versetzungen im krz-Gitter bei Nanoindentation wurden mit Hilfe des Potentials von Kimizuka et. al. [153] drei unterschiedliche Mechanismen gefunden (vgl. Abbildungsserie 30): Es können sich Versetzungsringe an der Oberfläche ausbilden, deren Durchmesser mit weiterer Verformung wächst; ein weiterer Mechanismus ist die Bildung von flächenartigen Fehlordnungen, welche sich beim weiteren Verformen in einen Versetzungsring umwandeln. Die Bildung dieser Fehlordnungen können sowohl an der Oberfläche als auch im Material erfolgen. Werden auch Leerstellen in der Simulation berücksichtigt, können diese auch Ausgangspunkt der Bildung von Versetzungsringen sein. Diese Ringe bilden sich dann unabhängig von der Oberfläche aus.

Bei der Simulation der Nanoindentation an reinem Eisen unter Verwendung des Potentials von Wen et. al. [136] können auch die typischen Abschnitte in der Kraft-Eindringkurve ausgemacht werden (vgl. Abbildung 33). Bei kleinen Eindringtiefen kann die Kurve mit dem Hertzschen Kontaktmodell (vgl. Kapitel 4.1) beschrieben werden. Dies deutet darauf hin, dass eine rein elastische Verformung vorliegt. Bei der Bildung der ersten Versetzungen, welche innerhalb des Materials stattfindet, folgt ein waagerechter Verlauf, der Pop-In. Sobald die Versetzungen bis zur Oberfläche gewachsen sind, endet der Pop-In. Im weiteren Verlauf der Simulation bilden sich neue Versetzungen aus, welche mit den vorhandenen interagieren. Es folgt der Übergang zum plastischen Verhalten.

Ein deutlicheres Bild zeigt die Bestimmung der Anzahl der aus der idealen krz Position verschobenen Atome (vgl. Abbildung 33). Hier zeigt sich eine sprunghafte Zunahme am Ende des elastischen Bereichs. Daran anschließend folgt eine in erster Näherung linearen Zunahme der Anzahl der aus der idealen krz Position verschobenen Atome. Die sprunghafte Zunahme wird als Pop-In interpretiert. Da jedoch dieser Sprung schon nach einer geringen Erhöhung der Indentereindringtiefe abgeschlossen ist, und aufgrund der Simulationsschwierigkeiten wird der Effekt in den Kraft-Eindringkurven nicht so deutlich sichtbar wie beim Palladium.

\section{Vergleich der Simulationen von Palladium und Eisen}

Der Vergleich der Simulationen von Palladium und Eisen zeigt, dass das aus der Versetzungstheorie [51] beschriebene Verhalten beobachtet werden kann: In kfz-Metallen bilden sich Versetzungen, indem sich Stapelfehler bilden und in krz-Metallen bilden sich vornehmlich Schraubenversetzungen aus.

Der Ort der Entstehung von Versetzungen ist uneinheitlich und im Falle von Eisen ändert sich das Verhalten während der Simulation. Die Simulationen an Palladium zeigen die Bildung von Versetzungsringen an der Oberfläche. Bei den Simulationen von Eisen mit dem Potential von Kimizuka et. al. [153] findet die Bildung zunächst an der Oberfläche und bei weiterer Verformung im Material statt. Die Simulationen von Eisen mit dem Potential von Wen et. al. [136] zeigen die Bildung von Versetzungen zentral unterhalb des Nanoindeters im Material.

Wird das Problem der Nanoindentierung kontinuumsmechanisch berechnet [151, 163], ergibt sich, dass die größte mechanische Spannung nicht direkt an der Oberfläche vorherrscht. Daher sollten generell bei der Nanoindentation Versetzungen im Material unterhalb des Nanoindenters entstehen. 
Bei den Simulationen wird dieses Verhalten nur bei Eisen unter der Verwendung des Potentials von Wen et. al. [136] beobachtet.

Im Folgenden wird die Tiefe $Z_{\tau_{\max }}$, in der die maximale Scherspannung $\tau_{\max }$ vorherrscht, für die verschiedenen Metalle berechnet. Die Tiefe $Z_{\tau_{\max }}$ ergibt sich kontiuumsmechanisch unter der Annahme einer ideal glatten Oberfläche wie folgt [163, 164]:

$$
Z_{\tau_{\max }}=0,48\left(\frac{3 P R_{S}}{4 E^{*}}\right)^{1 / 3}
$$

$P$ ist die auferlegte Kraft, $R_{S}$ ist der Radius des Indenters (bei den verwendeten Simulationen ist $R_{S}=10 \mathrm{~nm}$ ) und $E^{*}$ ist der reduzierte elastische Modul. Dieser ergibt sich aus dem elastischen Modul des Materials $E_{M}$ und dem elastischen Modul des Materials der Spitze $E_{S}$ unter Verwendung der jeweiligen Poissonzahlen $\nu_{M}$ und $\nu_{S}$, wie folgt:

$$
\frac{1}{E^{*}}=\frac{1-\nu_{M}^{2}}{E_{M}}+\frac{1-\nu_{S}^{2}}{E_{S}}
$$

Da der elastische Modul $E_{S}$ für die simulierte Nanoindenterspitze nicht bekannt ist, wird von einer idealen Spitze ausgegangen, d. h. $E_{S} \rightarrow \infty$ und $\nu_{S}=0$. Damit ergibt sich eine untere Abschätzung von $Z_{\tau_{\max }}$. Bei der Entstehung der ersten Versetzungen in den Simulationen liegt bei Eisen eine Kraft von $P_{C} \approx 1 \mathrm{mN}$ und bei Palladium $P_{C} \approx 0,3 \mathrm{mN}$ an. Damit ergibt sich für Eisen ${ }^{29}$ eine Tiefe von $Z_{\tau_{\max }}=3,3 \mathrm{~nm}$ und für Palladium ${ }^{30} Z_{\tau_{\max }}=2,5 \mathrm{~nm}$.

In der Simulation wird für Eisen unter Verwendung des Potentials von Wen et. al. [136] bei einer Nanoindentertiefe von $d=0,91 \mathrm{~nm}$ eine Entstehungstiefe der ersten Versetzungen von $Z=2,8 \mathrm{~nm}$ gefunden, vgl. Abbildung 31, und liegt somit im Bereich des theoretischen Werts. Da bei den anderen Simulationen Versetzungen an der Oberfläche entstehen, ergibt sich ein uneinheitliches Bild. Dies kann mehrere Gründe haben: Zum einen wird bei der theoretischen Herleitung der Tiefe $Z_{\tau_{\max }}[164]$ von einer ideal glatten Oberfläche ausgegangen. Dies ist bei einer Nanoindenterspitze mit einem Radius von $10 \mathrm{~nm}$ nicht mehr gegeben, da die Atomradien im Verhältnis zum Spitzenradius nicht mehr vernachlässigbar sind. Zudem wird in der kontinuumsmechanischen Beschreibung ein Spannungsfeld für ein isotropes Material berechnet. Dies ist bei der Simulation eines Einkristalls nicht gegeben. Hier muss die auferlegte Spannung im Bezug zu den einzelnen Kristallebenen betrachtet werden. Diese Gründe zeigen, dass eine kontinuumsmechanische Beschreibung der Simulationen nicht möglich ist.

\footnotetext{
${ }^{29}$ Verwendete Parameter für Eisen $E_{M}=200 \mathrm{GPa}, \nu_{M}=0,29$ [165]

${ }^{30}$ Verwendete Parameter für Palladium $E_{M}=121 \mathrm{GPa}, \nu_{M}=0,39$ [52]
} 


\section{Einfluss von Wasserstoff auf Nanoindentationsversuche}

Durch in Metall gelösten Wasserstoff ändert sich das prinzipielle Verhalten der (experimentellen) Kraft-Eindringkurve nicht. Die Kurven weisen einen elastischen Anfangsbereich, den Pop-In und daran anschließend einen plastischen Abschnitt auf.

Durch die Hinzugabe von Wasserstoff sinkt die Kraft, bei welcher der Pop-In auftritt. Dies gilt sowohl für Vanadium als auch für Palladium.

Dieses Verhalten wird durch das Defactant-Konzept [27, 28] vorhergesagt. Dadurch, dass Wasserstoff im Metall gelöst ist, wird die Linienenergie von Stufenversetzungen reduziert. Dies bedeutet, dass die Gesamtenergie eines Versetzungsrings, welcher sowohl aus Stufen- als auch Schraubenversetzungsanteilen zusammengesetzt ist, reduziert wird.

Für die weitere Bewegung der Versetzungen und damit für einen Pop-In, der durch ein Einsinken des Indenters sichtbar wird, ist es notwendig, dass Doppelkinken auf Versetzungen entstehen. Dies kann erklären, warum der Effekt bei Vanadium sehr ausgeprägt ist. Versetzungen in (reinem) Vanadium liegen größtenteils als Schraubenversetzungen vor [166]. Schraubenversetzungen im krz-Gitter sind im Gegensatz zu gemischten Versetzungen sehr unbeweglich. Die Bewegung von Schraubenversetzungen beruht auf der Bildung von Kinken, welche Stufencharakter haben. Ohne Wasserstoff ist die Bildung von Kinken sehr gehemmt, jedoch benötigt die Bewegung von Kinken auf einer Versetzung nur wenig Energie [55]. Dies führt zu geradlinigen Versetzungen, wodurch nur wenige Kinken auf der Versetzugslinie vorhanden sind.

Durch gelöste Fremdatome wird die Aktivierungsenergie von Doppelkinkenpaaren auf einer Schraubenversetzung reduziert [55] und somit wird die normalerweise blockierte Bewegungsmöglichkeit von Schraubenversetzungen aufgehoben. Der Grund hierfür liegt in der Reduzierung der Linienenergie von Stufenversetzungen, wenn Wasserstoff sich an der Versetzung anlagert [27, 28]. Palladium zeigt denselben Effekt in weniger ausgeprägter Form. Dies kann zum einen an der geringeren Wasserstoffkonzentration im Vergleich zum Versuch an Vanadium liegen, zum anderen spalten sich Versetzungen in partielle Versetzungen auf (siehe Kapitel 2.1), um ihre langreichweitigen Spannungen zu minimieren. Wasserstoff interagiert zwar auch mit partiellen Versetzungen [167], jedoch wird, anders als bei Vanadium, kein normalerweise gehemmter Verformungsmechanismus ermöglicht.

Der Vergleich des experimentellen Verhaltens zu dem Verhalten in Molekulardynamiksimulationen ist nur bedingt möglich. Bei den Simulationen war es nur im Fall von Palladium möglich einen Pop-In Effekt sowohl ohne als auch mit Wasserstoff anhand von Kraft-Eindringkurven nachzuweisen. Hier zeigt sich, dass es zu einer geringfügigen Reduzierung der Kraft kommt, bei der der Pop-In auftritt. Da der Unterschied der Kräfte jedoch kleiner ist als das Rauschen dieser Kurven, kann dieser Effekt auch zufälliger Natur sein.

Bei näherer Untersuchung des Einflusses von Wasserstoff auf die Bildung von Versetzungen konnten keine besonderen Vorgänge ausgemacht werden, also auch keine Anlagerung von Wasserstoff an den Versetzungen. Dies ist nicht überraschend, da die Gesamtsimulationszeit im Bereich von $t=0,1 \mathrm{~ns}$ liegt und der Wasserstoff so kaum Zeit hat, um zu günstigen Stellen zu diffundieren. Unter der Vorraussetzung, dass die Diffusion auf dieser Größenskala mit dem Diffusionskoeffizienten $D$ von Wasserstoff in Palladium in makroskopischen Proben beschreibbar ist, ergibt sich eine mittlere Diffusionslänge von $\sqrt{\left\langle x^{2}\right\rangle}=\sqrt{2 D t}=0,08 \mathrm{~nm}$. Auch wenn zusätzliche Simulationsschritte für Wasserstoff eingefügt werden (auf 100 normale Schritte folgten 10000 zusätzliche Schritte für Wasserstoff) ergibt sich eine mittlere Diffusionslänge von $\sqrt{\left\langle x^{2}\right\rangle}=0,8 \mathrm{~nm}$, was einer Distanz von 5 Zwischengitterplätzen entspricht. Jedoch wurden auch hier keine Besonderheiten bei der Bildung der Versetzungen auf atomarer Skala gefunden. Allerdings reichte die Simulationszeit aus, um einen von der Oberfläche unabhängigen Versetzungsring loszulösen. Zusammengefasst ergeben sich daher auch in den Simulationen von Palladium Anzeichen für eine Reduzierung der Linienenergie von Versetzungen durch Wasserstoff. 


\section{Auswirkungen von Wasserstoff auf die Bewegung von Versetzungen im ETEM}

In Anlehnung an frühere Experimente durch Birnbaum et. al. [25, 125] wurden Zugversuche an Palladium- und Vanadiumproben in Wasserstoffatmosphäre durchgeführt. Zur Beobachtung einzelner Versetzungen geschah dies in einem sog. environmental TEM (ETEM). Hierzu wurden die Proben bis zur Streckgrenze verformt, wodurch Versetzungsdynamik erzeugt wurde. Sobald die Versetzungsbewegung zum Erliegen gekommen war, wurde Wasserstoffgas ins TEM eingelassen. Es zeigte sich, dass sobald Wasserstoff hinzugegeben wurde, Versetzungen erneut anfingen sich zu bewegen. Diese Bewegung kam beim Erreichen eines konstanten Drucklevels erst nach und nach zum Erliegen. Wurde der Gasdruck erhöht, vollzog sich dieser Vorgang erneut. Dabei wurde für Palladium gezeigt, dass Versetzungen näher zueinander rücken. Dieses Verhalten ist vergleichbar zu früheren Arbeiten von Birnbaum et. al. [25, 125]. Diese Untersuchungen führten zur Formulierung des HELP-Mechanismus (engl.: Hydrogen-Enhanced Localised Plasticity) [21]. Dieses Modell besagt, dass Wasserstoff die Spannungsfelder von Versetzungen und anderen Defekten reduzieren, die somit besser auf von außen angelegte Spannungen reagieren können.

Die Versuche, welche im Rahmen dieser Arbeit durchgeführt wurden, zeigen, dass dieses Verhalten auch bei Palladium beobachtbar ist. Da für die Bewegung von Versetzung die Bildung von Kinken entscheidend ist (vgl. Kapitel 2.1), ist dies ein indirekter Hinweis darauf, dass die Interaktion von Wasserstoff mit Versetzungen die Bildungsenergie von Doppelkinkenpaaren, wie es im DefactantKonzept [27, 28] beschrieben wird, herabsetzt.

Die Untersuchung von Vanadium im ETEM (vgl. Abbildung 38 in Kapitel 5.1.3) zeigt, dass es durch Wasserstoff zum Wachstum eines vorhandenen Rissansatzes kommt. Gleichzeitig wurden viele Versetzungsbewegungen beobachtet.

Daraus wird gefolgert, dass auch hier die Lösung von Wasserstoff zur Reduzierung der Spannungsfelder von Versetzungen führt und Versetzungen einem äußeren Spannungsfeld besser folgen können. In diesem Fall ist das von außen auferlegte Feld durch den Riss gegeben. Die erhöhte Bewegung der Versetzungen führt zum Wachstum des Risses. Dies zeigt, dass unter Wasserstoffeinfluss Risse sich ausbreiten können, auch wenn die angelegte mechanische Spannung normalerweise für ein Risswachstum nicht ausreichen würde. Diese Beobachtung stellt also die Verbindung der mikrostrukturellen Vorgänge mit den makroskopischen Erscheinungen der Wasserstoffversprödung dar. 


\subsection{Einfluss von Wasserstoff auf Versetzungsnetzwerke}

Das Zusammenspiel vieler Versetzungen miteinander bei Verformung wurde im Rahmen dieser Arbeit anhand von Druckversuchen an Mikrosäulen aus Vanadium und anhand von TEM Untersuchungen an kaltgewalztem Palladiumblech untersucht. Des Weiteren wurde für die Untersuchung der Bildung von Korngrenzen Palladium durch HPT stark verformt.

Mit der Untersuchung von Säulen im Mikrometerbereich kann zum einen die Entstehung von einzelnen Versetzungen untersucht werden und zum anderen ist die Größe hinreichend groß, dass Versetzungen im Inneren miteinander interagieren können.

Die Untersuchung der Mikrostruktur, die beim Kaltwalzen entsteht, ist zum einen interessant, da es sich beim Walzen um eine häufig angewandte Methode handelt, die auch im industriellen Maßstab ihre Anwendungen findet, und zum anderen können mit ihr starke Verformung realisiert werden.

\section{Einfluss von Wasserstoff auf die Verformung von Vanadium-Mikrosäulen}

Die Untersuchungen an Vanadium-Mikrosäulen (vgl. Kapitel 5.2.1 bzw. [44]) zeigen, dass das Vanadium ohne Wasserstoff ein typisches Verhalten eines einkristallinen Metalls zeigt [168-170]. Die Säulen gleiten auf einzelnen $\{110\}$-Ebenen ab, welche mit den theoretischen Ebenen übereinstimmen, welche sich nach einer Schmidfaktoranalyse (siehe Kapitel 9.3 im Anhang) des verwendeten Belastungszustandes (die Säule ist in [001]-Richtung orientiert) ergeben. Die Erklärung, dass nur wenige Gleitebenen angeworfen werden, liegt in der Natur der Bildung von Versetzungen. Wandert eine Versetzung durch die Säule, bleibt an der Oberfläche eine atomare Stufe zurück. Die Bildung einer neuen Versetzung ist nun an dieser Stufe aufgrund der Geometrie begünstigt. Die Säule gleitet daher bevorzugt auf einer Ebene ab.

Allerdings zeigt sich eine Abweichung zum theoretischen Verhalten. Bei den Versuchen ohne und mit niedrigen Wasserstoffkonzentrationen $(0$ bis $0,0150 \mathrm{H} / \mathrm{V})$ erfolgt die Abgleitung nicht in den vorhergesagten $\langle 111\rangle$-Richtungen. Es zeigt sich, dass nur zwei gegenüberliegende Gleitsysteme an-

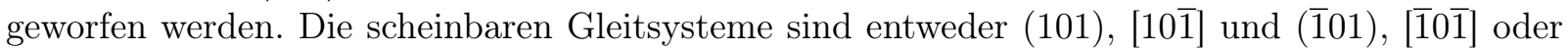

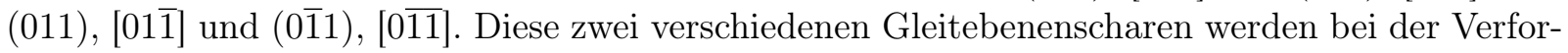
mung angeworfen, da die Säule am Grund durch die restliche Probe und an der Oberseite durch den Indenter in der Ebene fixiert ist. Dies erklärt die scheinbare Abweichung der Abgleitrichtung vom Idealverhalten. Ein ideales Gleitsystem im krz Gitter besteht aus einer $\{110\}$ Ebene und kann auf zwei zur Ebenennormale senkrechten $\langle 111\rangle$ Richtungen abgleiten. Es wird angenommen, dass bei diesen Versuchen die Abgleitung zunächst auch in eine dieser Richtungen erfolgt. Da jedoch die Säule fixiert ist, baut sich eine rücktreibende Kraft auf, wodurch die Abgleitrichtung geändert wird. Die Säule gleitet also in einer Zickzack-Bewegung ab.

Bei der Zugabe von Wasserstoff ergibt sich ein zunächst widersprüchliches Ergebnis: Die Versuche zeigen, dass die Verformung von Vanadium-Mikrosäulen mehr Energie benötigt, wenn Wasserstoff in Vanadium gelöst ist, vgl. Abbildung 39. Dieser Widerspruch lässt sich auf die Reduzierung der Linienenergie durch Wasserstoff zurückführen. Beim Verformen werden aufgrund der Reduzierung der Linienenergie durch Wasserstoff mehr Gleitsysteme für die Verformung angeworfen. Die Säule verformt sich unter Verwendung aller vier Gleitebenenscharen. Folglich kommt es im Laufe der Verformung dazu, dass Versetzungen auf unterschiedlichen Gleitsystemen miteinander interagieren müssen. Passiert dies, benötigt dieses zum einen Energie und zum anderen kann durch die Interaktion von Versetzungen im krz Gitter eine immobile Schraubenversetzung zurückbleiben [57, 171]. Daher kann eine Gleitebene nur für wenige Versetzungen verwendet werden. Dies kann im Experiment anhand von feinen Stufen auf der Oberfläche nachvollzogen werden, vgl. Abbildung 41. Makroskopisch nimmt die Säule bei Anwesenheit von Wasserstoff durch Verformung die Form eines Fasses an. Des Weiteren führt die Interaktion von Versetzungen dazu, dass Versetzungen in der Säule verbleiben. Es kommt zur Erhöhung der Versetzungsdichte und dies führt zu einer Verfestigung des Materials. 


\section{Versetzungsbildung in kaltgewalztem Palladiumblech}

Für die Untersuchung des Einflusses von Wasserstoff auf die Bildung von Versetzungsnetzwerken in Palladium (Kapitel 5.2.2) wurden nahezu versetzungsfreie Palladiumbleche mit unterschiedlichen Konzentrationen an Wasserstoff beladen und anschließend auf die gleiche Art und Weise kaltgewalzt. Dabei wurden verschiedene Konzentrationen innerhalb des Einphasengebiets des Palladium-Wasserstoffsystems ( $\alpha$-Phase) gewählt, um zusätzliche Effekte durch das Ausbilden einer Hydridphase auszuschließen.

TEM-Aufnahmen davon (Abbildungsserie 42) zeigen eine drastische Zunahme der Versetzungsdichte. Ohne Wasserstoff (Abbildung 42a) bilden sich sog. Versetzungszellen aus. Dies sind versetzungsfreie Bereiche, welche durch Versetzungsnetzwerke voneinander getrennt werden. Bereits die Lösung einer kleinen Konzentration von $0,001 \mathrm{H} / \mathrm{Pd}$ (Abbildung 42b) verursacht die Entstehung von so vielen Versetzungen, dass keine freien Bereiche in den TEM-Aufnahmen mehr erkennbar sind. Bei weiterer Erhöhung der Wasserstoffkonzentration bilden sich wieder Versetzungezellen, jedoch mit dichteren Zellwänden. Bei hohen Konzentrationen (ab 0,01 H/Pd, Abbildung 42d) innerhalb der $\alpha$-Phase bilden sich sog. Subkörner aus. Dabei handelt es sich um versetzungsarme Bereiche, welche durch sehr versetzungsreiche Zellwände voneinander getrennt sind. Dies führt dazu, dass die einzelnen Subkörner gegeneinander verkippt sind, was aus der Variation der Helligkeiten der Körner in den TEM-Aufnahmen geschlossen werden kann.

Die beim Kaltwalzen entstehende Versetzungsdichte wurde in der Diplomarbeit von Hans Peter Barth ${ }^{31}$ [172] in Zusammenarbeit mit Dr. Yuzeng Chen ${ }^{32}$ untersucht und in den Arbeiten [40,43] veröffentlicht. In Abbildung 48 ist die beim Kaltwalzen entstehende Versetzungsdichte als Funktion des gelösten Wasserstoffs in der Probe dargestellt [40]. Die Versetzungsdichte wurde hierzu auf zwei verschiedene Arten bestimmt: Für diese Untersuchungen wurde die Methode von Williamson und Hall [173] verwendet. Dabei wird die Verbreiterung von RöntgenBeugungsreflexen durch Verzerrungen analysiert. Wird angenommen, dass die Verzerrung hauptsächlich durch Versetzungen hervorgerufen wird, kann die Versetzungsdichte ermittelt werden.

Des Weiteren wurde eine eigens für diese Untersuchungen entwickelte Diffusionsmethode verwendet. Diese erlaubt eine völlig unabhängige Bestimmung der relativen Versetzungsdichte. Hierbei wird das Diffusionsverhalten von Wasserstoff ausgenutzt. Nach der Verformung wird der Wasserstoff zunächst aus der Probe entfernt. Danach wird die Probe in einem sog. Doppelzellaufbau untersucht. Hier wird von einer Seite der Probe definiert neuer Wasserstoff in kurzen Stößen in der Probe gelöst. Der Wasserstoff diffundiert durch die Probe und wird auf der anderen Seite detektiert. Dadurch kann die Diffusionskonstante des Wasserstoffs in Palladium bestimmt werden. Mit jedem Beladepuls wird die Wasserstoffkonzentration erhöht, somit kann die Diffusionskonstante als Funktion der Wasserstoffkonzentration ermittelt werden. Der Wert der Diffusionskonstante wird reduziert, wenn Wasserstoff attraktiv mit Kristallbaufehlern interagiert; der Wasserstoff verweilt länger an den Defekten. Die Konzentration des mit Kristallbaufehlern interagierenden Wasserstoffs wird gebundener Wasserstoff genannt und kann aus der Diffusionskonstante bestimmt werden und ist eine Funktion des chemischen Potentials und der Versetzungsdichte. Ein Vergleich des gebundenen Wasserstoffs von zwei verschiedenen Proben bei gleichem chemischen Potential ergibt die relative Versetzungsdichte zwischen den Proben. Eine genaue Beschreibung des Verfahrens ist in der Veröffentlichung [40] zu finden.

\footnotetext{
${ }^{31}$ Institut für Materialphysik, Univeristät Göttingen

${ }^{32}$ State Key Lab of Solidification Processing, Northwestern Polytechnical University, Xián, People's Republic of China
} 


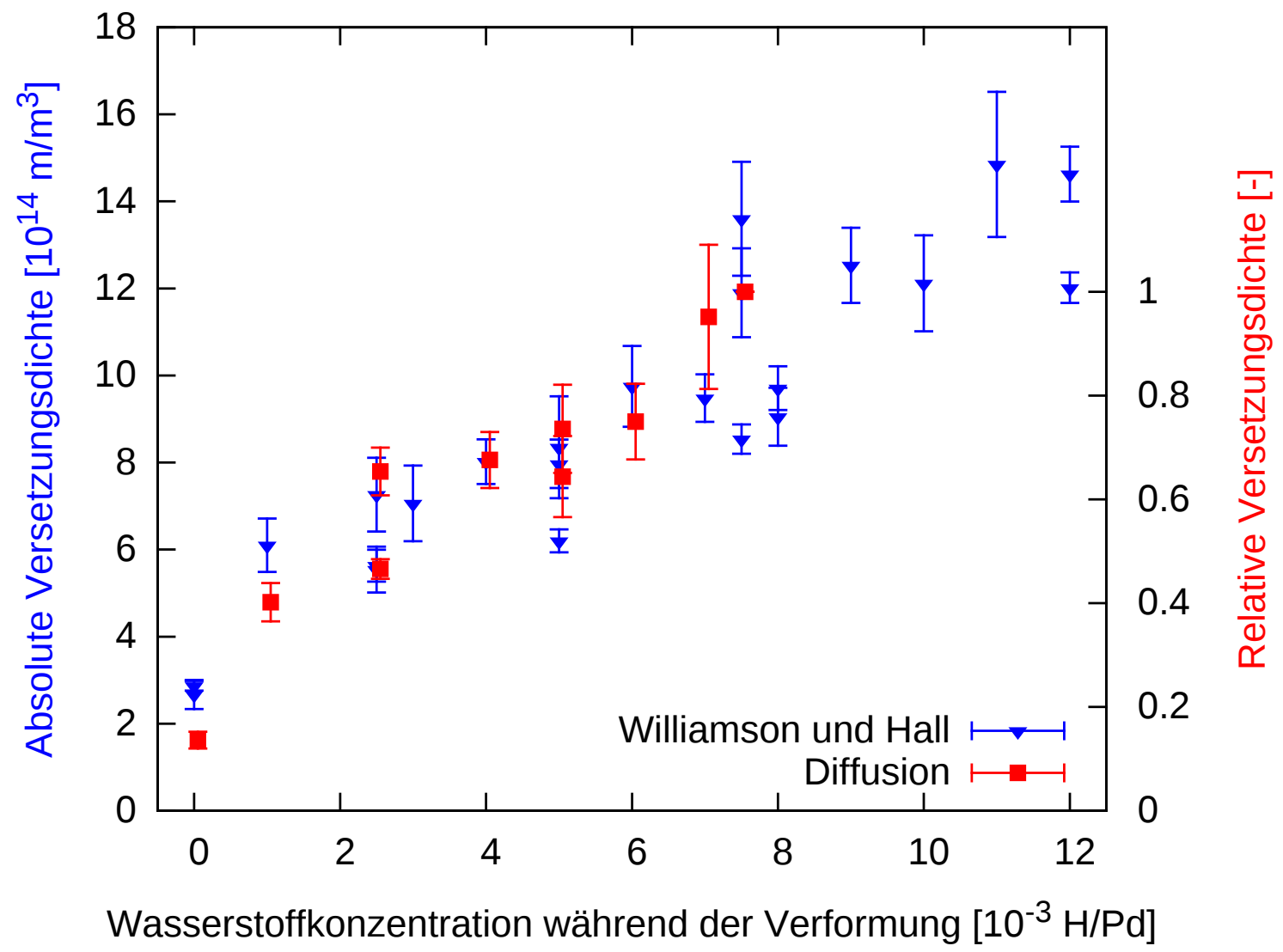

Abb. 48 Entwicklung der Versetzungsdichte von kaltgewalztem Palladium mit zunehmender Wasserstoffkonzentration während der Verformung [40]. Dabei wurden zwei unabhängige Methoden verwendet: Zum einen wurde die Methode von Williamson und Hall [173], bei der die Verbreiterung von Reflexen in Röntgenbeugungsdiagrammen untersucht wird, verwendet. Zum anderen wurde durch die Messung der Diffusion die relative Versetzungsdichte ermittelt. Dabei wurde die relative Versetzungsdichte zur Probe, welche mit 0,0075 H/Pd verformt wurde, bestimmt und anhand dieser auch im Graphen ausgerichtet.

Das in den TEM-Bildern von kaltgewalztem Palladium gesehene Verhalten kann in der Auftragung der Versetzungsdichte in Abbildung 48 nachvollzogen werden. Bereits eine kleine Konzentration in der $\alpha$-Phase des Palladium-Wasserstoffsystems von $0,001 \mathrm{H} / \mathrm{Pd}$ führt zur Verdoppelung der Versetzungsdichte im Vergleich zum wasserstofffreien Palladium. Wird die Wasserstoffkonzentration beim Walzen weiter erhöht, steigt auch die Versetzungsdichte linear mit einer Steigung von $(7,2 \pm 0,8) \cdot 10^{16} \mathrm{~m}^{-2}\left(\frac{\mathrm{H}}{\mathrm{Pd}}\right)^{-1}$ an.

Zusammengefasst zeigen die Beobachtungen an kaltgewalztem Palladium zwei Effekte, die durch Wasserstoff hervorgerufen werden: Die TEM-Bilder zeigen, dass es zur Bildung von Versetzungszellen und bei hohen Konzentrationen zur Bildung von Subkörnern kommt. Gleichzeitig steigt die Versetzungsdichte mit zunehmender Wasserstoffkonzentration beim Walzen. Dies bedeutet, dass in den Zellwänden die Versetzungen mit steigender Wasserstoffkonzentration dichter zusammenrücken. Dieses Ergebnis zeigt im Vergleich zu den ETEM Ergebnissen auf eine andere Art die Reduzierung der Spannungsfelder von Versetzungen durch Wasserstoff, wodurch die Versetzungen dichter zusammenrücken können.

Des Weiteren wurde gezeigt, dass die Versetzungsdichte bei Anwesenheit von Wasserstoff deutlich erhöht wird. Dies ist also ein weiteres Anzeichen dafür, dass die Linienenergie durch die Anwesenheit von Wasserstoff reduziert wird. 


\section{HPT Verformung von Palladium-Wasserstofflegierungen}

In dieser Arbeit wurden aufbauend auf Untersuchungen [48, 156, 157] in der Arbeitsgruppe von Prof. Dr. M. Zehetbauer an der Universität Wien HPT-Verformungen an Palladium-Wasserstofflegierungen durchgeführt. Dazu wurden von den verformten Palladiumproben TEM-Aufnahmen angefertigt, welche eine nanokristalline Kornstruktur bei jeder untersuchten Wasserstoffkonzentration zeigen. Der mittlere Korndurchmesser bei den Proben, welche in der $\alpha$-Phase des PalladiumWasserstoffsystems bei Raumtemperatur verformt wurden, ergibt sich zu $D=200 \mathrm{~nm}$. Bei einer kleinen Konzentration im Zweiphasengebiet von $0,2 \mathrm{H} / \mathrm{Pd}$ reduziert sich der mittlere Korndurchmesser auf $D=100 \mathrm{~nm}$ und viele Versetzungen sind zu erkennen. Eine Kornverkleinerung durch Hinzugabe von Fremdatomen wird auch bei anderen Verformungsmethoden beobachtet [37]. Um einen Wert für den gebundenen Wasserstoff an den Korngrenzen zu ermitteln, wird wie folgt vorgegangen: Nach [28] wurde eine Konzentration von $c_{t}=0,11 \mathrm{H} / \mathrm{Pd}$ an gebundenen Wasserstoff für nanokristallines Palladium mit einer Korngröße von $D=22 \mathrm{~nm}$ im Zweiphasengebiet ermittelt. Der Excess $\Gamma$ von Wasserstoff an den Korngrenzen kann wie folgt ausgedrückt werden:

$$
\Gamma=c_{t} \cdot \frac{K_{V} D^{3}}{\Omega_{H}} \cdot \frac{1}{K_{O} D^{2}}
$$

Hierbei ist $K_{V}$ ein Formfaktor, um das Volumen zu bestimmen, $\Omega_{H}$ das molare Volumen des Wasserstoffs und $K_{O}$ ein Formfaktor, um die Oberfläche eines Korns zu bestimmen. Wird angenommen, dass $\Gamma$ und die Formfaktoren $K_{V}$ und $K_{O}$ unabhängig von der Korngröße sind, ergibt sich die gebundene Wasserstoffkonzentration $c_{t}$ für die Korndurchmesser $D=100 \mathrm{~nm}$ und $D=200 \mathrm{~nm}$, wie folgt:

$$
\begin{aligned}
& c_{t, 100 \mathrm{~nm}}=0,025 \mathrm{H} / \mathrm{Pd} \\
& c_{t, 200 \mathrm{~nm}}=0,012 \mathrm{H} / \mathrm{Pd}
\end{aligned}
$$

Dies zeigt, dass zwar eine doppelt so hohe zusätzliche Konzentration von $\Delta c_{t}=c_{t, 100 \mathrm{~nm}}-$ $c_{t, 200 \mathrm{~nm}}=0,013 \mathrm{H} / \mathrm{Pd}$ an den kleineren Körner gebunden werden kann, diese Konzentration ist jedoch immer noch klein gegenüber der Gesamtkonzentration. Daher kann der eingebrachte Wasserstoff nicht alleinig durch die Korngrenzen aufgenommen werden. Der Wasserstoff liegt somit gelöst in der $\alpha$ - und als Teil der $\alpha^{\prime}$-Phase des Palladiums vor. Des Weiteren wurde von Krystian et. al. [48] gezeigt, dass es bei der Verformung von Palladium-Wasserstofflegierungen mit HPT zur Bildung s.g. SAV (engl. superabundant vacancies) kommt. Dies sind Leerstellen, welche durch Wasserstoff stabilisiert werden. Bei den Untersuchungen von Krystian et. al. [48] entstehen bei der Verformung sehr hohe Konzentrationen zwischen $1,3 \cdot 10^{-2}$ und $4,8 \cdot 10^{-2}$ Leerstellen pro Palladium Atom.

Eine deutliche Änderung des Verhaltens beim Verformen wird erst bei einer Konzentration von $0,45 \mathrm{H} / \mathrm{Pd}$ beobachtet. Bei dieser hohen Konzentration im Zweiphasengebiet entstehen neben wenigen kleinen Körnern mit einem mittleren Durchmesser von $D=200 \mathrm{~nm}$, große unregelmäßige Körner mit Durchmessern im Mikrometerbereich. Zusätzlich liegt eine sehr hohe Versetzungsdichte vor.

Für eine ähnliche Abschätzung des gebundenen Wasserstoffs an Versetzungen ergibt sich bei der Annahme einer sehr hohen Versetzungsdichte von $\rho=10^{16} \mathrm{~m} / \mathrm{m}^{3}$ nach [63] eine maximale Konzentration an gebundenem Wasserstoff $c_{t, \rho}$ von

$$
c_{t, \rho}=0,037 \mathrm{H} / \mathrm{Pd}
$$

Beide Abschätzungen zeigen, dass nur ein Bruchteil des gelösten Wasserstoffs zur Bildung neuer Defekte verwendet wird und somit der restliche Wasserstoff in der Hydridphase ( $\alpha^{\prime}$-Phase) oder an SAVs gebunden ist. Jedoch zeigen diese Versuche, dass Wasserstoff auch bei dieser extremen Verformung einen enormen Einfluss auf die Defektbildung hat. 


\subsection{Einfluss von Wasserstoff auf die Linienenergie}

Um den Einfluss von Wasserstoff auf die Bildung von Versetzungen zu quantifizieren, kann die Linienenergie der Versetzungen bestimmt werden. Dazu eignen sich zum einen die Untersuchungen durch Nanoindentation und zum anderen die Untersuchungen am Palladiumblech.

\section{Bestimmung der Linienenergie aus Nanoindentationsversuchen}

Wie bereits im Kapitel 6.1 diskutiert hängt die Reduzierung der Kraft, bei dem der Pop-In Effekt $P_{C}$ auftritt, von der Linienenergie $E_{L}$ der Versetzungen ab. Die Linienenergie kann wie folgt bestimmt werden [95]:

$$
P_{C}=\left(\frac{E_{L}}{0,31}\right)^{3} \frac{4 \pi^{3} R_{S}^{2}}{3 E^{* 2} b^{3} r_{c}^{3}}
$$

Hierbei ist $E^{*}$ der reduzierte elastische Modul (siehe Gleichung 6.2), $b$ der Burgersvektor und $r_{c}$ ist die Versetzungsringgröße bei der Nukleation. $R_{S}$ ist der Spitzenradius des Nanoindenters, welcher nicht bestimmt werden konnte. Unter der Voraussetzung, dass der elastische Modul nicht durch Wasserstoff beeinflusst wird, kann der relative Unterschied der Linienenergie $r_{E_{L}}$ zwischen zwei Untersuchungen mit verschiedenen Wasserstoffkonzentrationen (1 und 2) wie folgt bestimmt werden:

$$
r_{E_{L}}=1-\left(\frac{P_{C, 1}}{P_{C, 2}}\right)^{1 / 3}
$$

Unter Verwendung der Ergebnisse zur Kraft, bei der der Pop-In Effekt auftritt, aus Kapitel 5.1.1 Abbildung 24 ergeben sich für Vanadium die Werte in Abbildung 49.

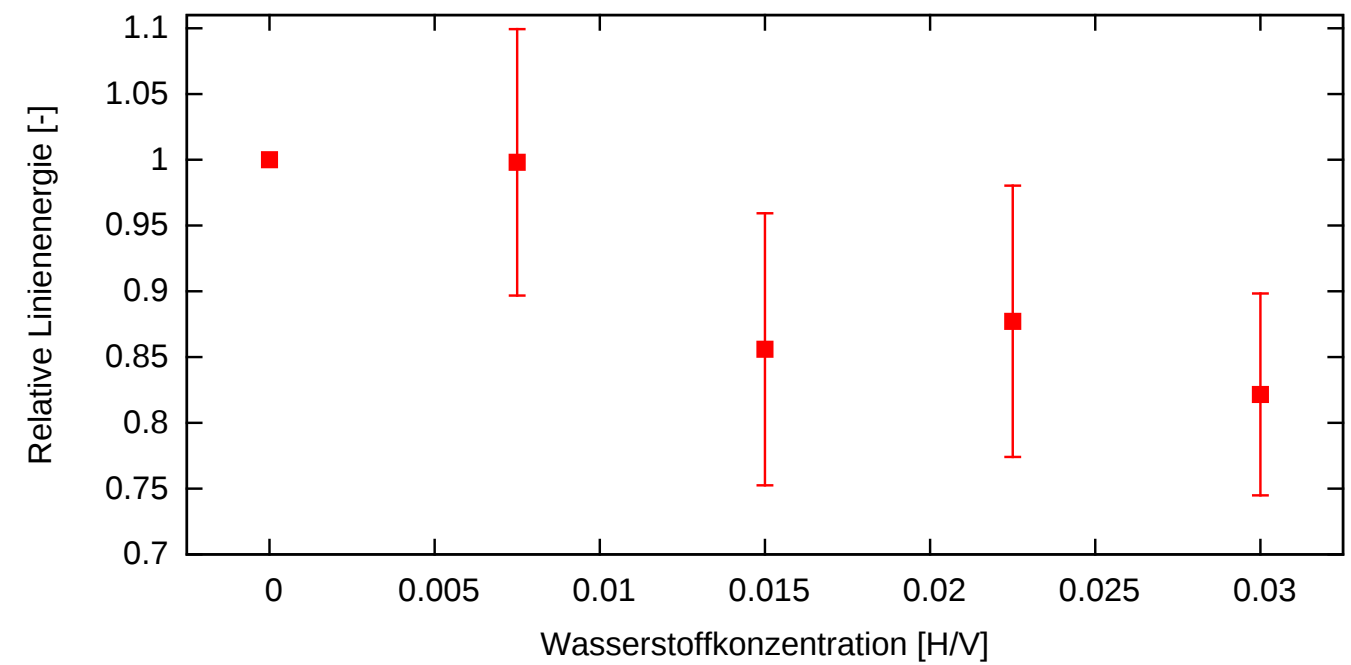

Abb. 49 Einfluss von Wasserstoff auf die Linienenergie von Versetzungen in Vanadium, ermittelt aus Nanoindentation. Dargestellt ist die relative Abnahme der Linienenergie bezogen auf die Ergebnisse der Nanoindentation ohne Wasserstoff.

Zusammengefasst ergibt sich eine Reduzierung der Linienenergie bei Vanadium durch das Hinzufügen von $0,03 \mathrm{H} / \mathrm{V}$ um $18 \%$.

Die gleiche Vorgehensweise bei Palladium zeigt, dass die Linienenergie durch Hinzufügen von $0,005 \mathrm{H} / \mathrm{Pd}$ um $11 \%$ reduziert wird. 


\section{Bestimmung der Linienenergie aus der Versetzungsdichte in kaltgewalztem Palladium}

Die Ergebnisse zur Versetzungsdichte können zur Abschätzung der Linienenergie der Versetzungen verwendet werden [172]. Dazu muss angenommen werden, dass, nachdem der Wasserstoff aus der Probe wieder entfernt wurde, die Versetzungen nicht wieder annihilieren. Dies kann vorrausgesetzt werden, da eine sehr hohe Versetzungsdichte vorliegt und dadurch die Versetzungen durch Interaktion miteinander in ihrer Bewegung eingeschränkt sind. Zudem zeigen Dynamische Differenzkalorimetrie Studien [48], dass Versetzungen in Palladium erst oberhalb von $480 \mathrm{~K}$ ausheilen. Die im Defactant-Konzept vorhergesagte Reduzierung der Linienenergie durch die Anlagerung von Wasserstoff an Versetzungen kann ausgehend von der Versetzungsdichte wie folgt abgeschätzt werden: Die Linienenergie einer Stufenversetzung $E_{L}$ ergibt sich nach Gleichung $2.3 \mathrm{zu}$ [172]:

$$
E_{L}=\frac{G b^{2}}{4 \pi(1-\nu)} \ln \left(\frac{R}{r_{0}}\right)
$$

Da nur Stufenversetzungen im kfz Gitter über ein langreichweitiges hydrostatisches Spannungsfeld verfügen, wird angenommen, dass Wasserstoff sich nur an Versetzungen mit Stufencharakter anlagert. Des Weiteren wird angenommen, dass der Schermodul $G=44 \mathrm{GPa}$ [52] nicht beeinflusst wird (Dies kann unter Umständen nicht pauschal angenommen werden $[22,174,175]) . r_{0}$ ist der innere Radius des Versetzungskerns und ist in der Größenordnung des Burgersvektor $b=0,275$ nm und $\nu=0,39$ ist die Poissonzahl [52]. $R$ ist die Reichweite des Spannungsfeldes einer Stufenversetzung. Bei hohen Versetzungsdichten $\rho$, wie sie in diesen Versuchen vorliegen, kann die Reichweite durch $R \approx \frac{1}{\sqrt{\rho}}$, also den mittleren Abstand zwischen zwei benachbarten Versetzungen, genähert werden. Damit ergibt sich die Linienenergie zu [172]:

$$
E_{L}=\frac{G b^{2}}{4 \pi(1-\nu)} \ln \left(\frac{1}{b \sqrt{\rho}}\right)
$$

Die Linienenergie von kaltgewalztem Palladium ohne Wasserstoff unter Verwendung der Versetzungsdichte, welche mit der Analyse der Röntgenbeugungsdiagramme nach der Methode von Williamson und Hall bestimmt wurde, ergibt sich damit zu:

$$
E_{L, 0}=2 \times 10^{-9} \frac{\mathrm{J}}{\mathrm{m}}=3 \frac{\mathrm{eV}}{\mathrm{b}}
$$

Um eine unabhängige Berechnung der Linienenergie zu ermöglichen, kann auch die relative Versetzungsdichte $\left(\frac{\rho_{A}}{\rho_{B}}\right)$ zwischen zwei Proben $A$ und $B$, welche mit der Diffusionsmethode in $[40,172]$ ermittelt wurde, verwendet werden:

$$
\Delta E_{L}=E_{L}\left(\rho_{A}\right)-E_{L}\left(\rho_{B}\right)=\frac{G b^{2}}{8 \pi(1-\nu)} \ln \left(\frac{\rho_{B}}{\rho_{A}}\right)
$$

Die Abbildung 50 zeigt die berechneten Linienenergien, die ausgehend von der Versetzungsdichte bestimmt wurden.

Die Linienenergie für die Probe, welche bei bspw. $c_{\mathrm{H}}=0,0075 \mathrm{H} / \mathrm{Pd}$ verformt wurde, ist um $16 \%$ (Röntgenmethode) bzw. $23 \%$ (Diffusionsmethode) reduziert. 


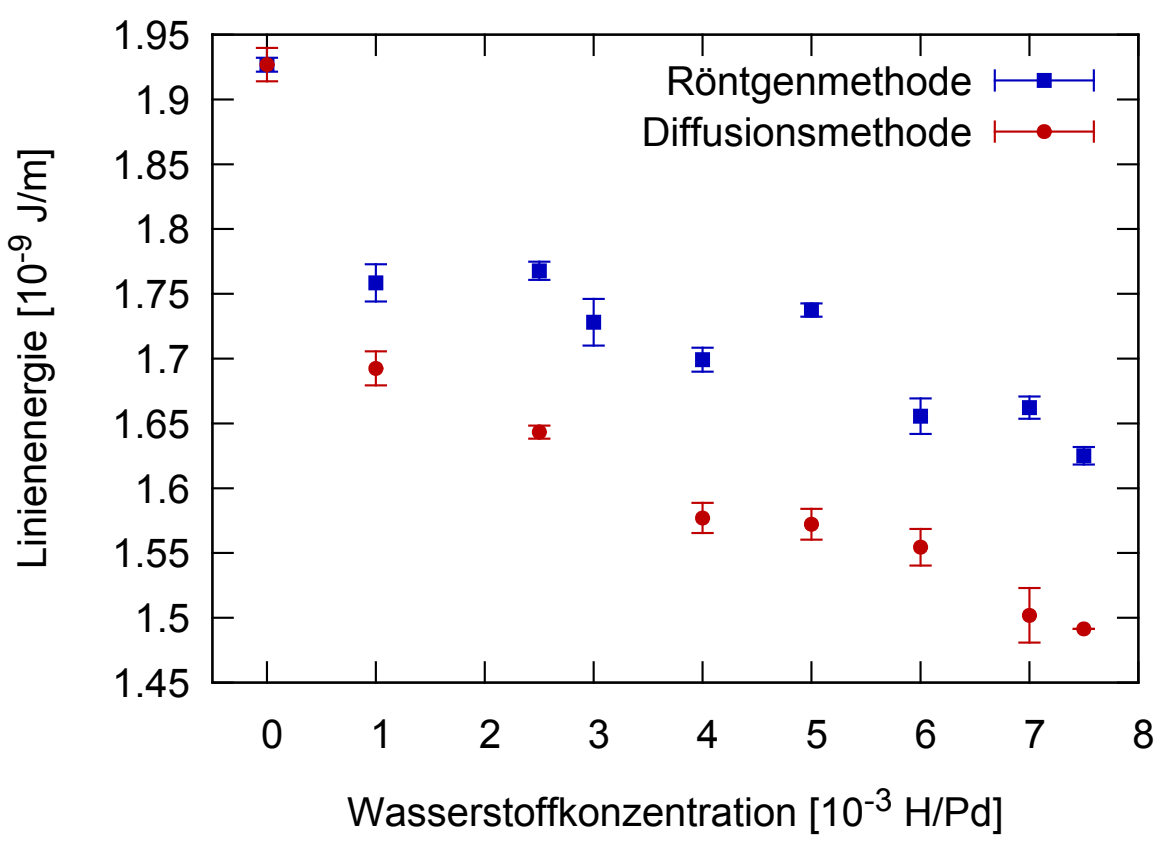

Abb. 50 Einfluss von Wasserstoff auf die Linienenergie von Versetzungen in kaltgewalztem Palladium ermittelt aus der Versetzungsdichte. Die relativen Werte der Linienenergie, die mit der Diffusionsmethode bestimmt wurden, sind bezogen auf den Wert, der sich bei einer Verformung ohne Wasserstoff ergibt. Für die Darstellung sind die relativen Werte der Linienenergie, welche sich aus der Diffusionsmethode ergeben, in absolute Werte umgerechnet worden.

\section{Einfluss von Wasserstoff auf die Linienenergie von Versetzungen}

Die hier vorgestellten Versuche zeigen, dass Wasserstoff die Linienenergie in den untersuchten Metallen deutlich reduzieren kann. Für Palladium konnte die Reduzierung der Linienenergie durch Wasserstoff mit drei unabhängigen Methoden bestimmt werden.

So wurde bei den Nanoindentationsversuchen durch Hinzugabe einer Wasserstoffkonzentration von $0,005 \mathrm{H} / \mathrm{Pd}$ eine Reduzierung der Linienenergie um $11 \%$ bestimmt. Für die gleiche Wasserstoffkonzentration ergibt sich durch Auswertung der Versetzungsdichte eine Reduzierung von 10\% (Röntgenmethode) bzw. 18\% (Diffusionsmethode).

Die maximale Reduzierung der Linienergie von Versetzungen wurde anhand von kaltgewalztem Palladiumblech festgestellt. Hier ergibt sich eine Reduzierung der Linienenergie von Versetzungen bei einer Probe, welche bei einer Wasserstoffkonzentration von $c_{\mathrm{H}}=0,0075 \mathrm{H} / \mathrm{Pd}$ verformt wurde, mit 16\% (Röntgenmethode) bzw. $23 \%$ (Diffusionsmethode). Diese Ergebnisse sind vergleichbar zu anderen Untersuchungen des Einflusses von Wasserstoff auf die Linienenergie von Versetzungen. Bspw. zeigt Palladium eine maximale Reduzierung der Linienenergie von Versetzungen von $26 \%$ [28] ermittelt durch Messungen der EMK von verschieden verformten Palladiumproben bzw. von $33 \%$ [62] gemessen durch Kleinwinkelneutronenstreuung an mehrmals bis in die Hydridphase beladenem und entladenem Palladium. Dieser Effekt ist nicht nur auf Palladium und Vanadium beschränkt. So wurde bspw. bei Nanoindentationsuntersuchungen an FeAl-Einkristallen eine maximale Reduzierung der Linienenergie von $27 \%$ gefunden [176].

All diese Untersuchungen zeigen, dass die Reduzierung der Linienenergie von Versetzungen ein genereller Effekt von gelöstem Wasserstoff ist. 


\subsection{Zusammenfassung der beobachteten Einflüsse von Wasserstoff auf die Defektbildung}

Alle in dieser Arbeit beobachteten Vorgänge basieren auf der Reduzierung der Linienenergie bzw. auf der Reduzierung der Interaktionsenergie von Versetzungen durch die Lösung von Wasserstoff in Metall. Der Grund für die Reduzierung ist die energetisch positive Interaktion von gelösten Fremdatomen mit Kristalldefekten, wie es im Defactant-Konzept [27, 28] beschrieben wird. Gelöste Atome und auch Kristalldefekte verspannen das Kristallgitter. Können die gelösten Atome sich bspw. durch Diffusion an den Defekten anlagern reduzieren diese ihre Energie im Gitter. D. h. die Freie Energie eines Materials kann reduziert werden dadurch, dass es zur lokalen Erhöhung der Konzentration an Defekten kommt. Wird also eine Konzentration an Fremdatomen in ein defektfreies Material eingebracht, kann die Bildung von neuen Kristalldefekten zur Reduzierung der Freien Energie führen. Folglich interpretiert das Defactant-Konzept diese Energiereduzierung aus der Sicht der Defekte, indem die Energiereduzierung als Defektenergiereduzierung interpretiert wird. Diese Erscheinung gilt nicht nur für die in dieser Arbeit gezeigte Reduzierung der Linienenergie von Versetzungen, sondern für alle Arten von Kristalldefekten. So kommt es bei der Lösung von enormen Mengen von Wasserstoff in Palladium zur Bildung von ungewöhnlich hohen Leerstellenkonzentrationen. Dies passiert entweder durch hohe Wasserstoffdrücke bei hohen Temperaturen [47] oder durch extreme Verformung [48]. Dieses Phänomen ist nicht nur auf Wasserstoff in Palladium beschränkt. Ein weiteres Beispiel für die Erhöhung der Defektbildung ist das Kugelmühlen von Eisen mit Kohlenstoff [37]. Hier zeigt sich eine Kornverfeinerung, also eine Vergrößerung der Oberfläche der Körner, durch die Hinzugabe von Kohlenstoff beim Mahlvorgang.

\section{Auswirkungen der Reduzierung der Linienenergie von Versetzungen}

Die Reduzierung der Linienenergie von Versetzungen führt bei Nanoindentationsversuchen zum Absinken der Kraft beim Pop-In Effekt und bei der Verformung von Vanadium-Mikrosäulen dazu, dass die Säulen mit gelöstem Wasserstoff einer höheren mechanischen Belastung widerstehen. Dieses zunächst widersprüchlich erscheinende Verhalten kann dadurch erklärt werden, dass durch gelösten Wasserstoff mehr Versetzungen gleichzeitig in verschiedenen Gleitsystemen entstehen. Diese bewegen sich durch die Säule und treffen dabei auf andere Versetzungen in anderen Gleitsystemen. Passiert dies, benötigt diese Interaktion zum einen Energie und zum anderen kann dadurch eine immobile Schraubenversetzung im krz Gitter zurückbleiben [57, 171]. Es kommt zur Erhöhung der Versetzungsdichte und dies führt dazu, dass das Material schwieriger zu verformen ist. Dies führt außerdem dazu, dass eine Gleitebene nur für wenige Versetzungen verwendet werden kann. Erkennbar ist dies im Experiment anhand von feinen Stufen auf der Oberfläche der verformten Säulen, vgl. Abbildung 41. Makroskopisch nimmt die Säule bei Anwesenheit von Wasserstoff durch Verformung die Form eines Fasses an.

Am Beispiel der Untersuchungen im ETEM konnte gezeigt werden, dass Versetzungen durch gelösten Wasserstoff dichter aneinander rücken, um dem von außen auferlegten Spannungsfeld besser zu folgen. Diese direkte Beobachtung unterstreicht die Ergebnisse am gewalzten Palladium, bei denen indirekt der Effekt der Reduzierung des Abstandes von Versetzungen durch die Bildung von Versetzungszellen gezeigt wurde. Dies wird im Rahmen des HELP Modells [21] damit erklärt, dass die mechanischen Spannungen ausgehend von Versetzungen durch Wasserstoff reduziert werden. Diese Reduzierung der Spannungsfelder kann damit erklärt werden, dass Versetzungen eine erhöhte Beweglichkeit haben. Dies erlaubt es ihnen besser eine optimale Position zueinander einzunehmen. Die Beweglichkeit beruht auf der Bildung von Kinken auf der Versetzungslinie (vgl. Kapitel 2.1). Diese Bildung von Kinken und die Bildung von Versetzungszellen in Palladium wird zum einen durch die Reduzierung der Linienenergie von Versetzungen und zum anderen durch die Reduzierung der Spannungsfelder von Versetzungen begünstigt. Dies führt bei hohen Konzentrationen $(c=0,01 \mathrm{H} / \mathrm{Pd}$, vgl. Abbildung 42d) im Einphasengebiet des PalladiumWasserstoffsystems so weit, dass sich Subkörner bilden. Bei massiverer Verformung (vgl. Kapitel 5.2.3) wird dieses Verhalten fortgeführt und es kommt zur Bildung eines nanokristallinen Gefüges. 


\section{5 Übergang zu makroskopischen Vorgängen ausgelöst durch gelösten Wasserstoff}

Die Lösung von Wasserstoff und damit einhergehend die Reduzierung der mechanischen Belastbarkeit kann in Konstruktionen aus Metall eine verheerende Auswirkung haben [177]. Ein häufig auftretendes Problem ist der Eintrag von Wasserstoff in Metallprodukte bei der Herstellung. Oft werden diese Produkte mit einer elektrolytisch aufgetragenen Beschichtung gegen Oxidation geschützt. Beim Aufbringen kann es zur Lösung von Wasserstoff im Metall kommen. Dies führt entweder direkt zur Bildung von Oberfächenschäden oder bleibt zunächst unbemerkt und kann zum frühzeitigen Versagen des Produktes in der Verwendung führen, indem die Rissbildung gefördert wird.

Um diese Vorgänge zu untersuchen wurden im Rahmen dieser Arbeit zwei Bachelorarbeiten betreut, die die makroskopischen Auswirkungen von Wasserstoff auf die mechanischen Eigenschaften untersucht haben. In der Arbeit von Annegret Lehmberg [178], siehe auch Kapitel 9.4.1 im Anhang, wurde untersucht, welche Auswirkungen eine extreme Wasserstoffbeladung von Eisen hat, wie sie bei elektrolytischer Beschichtung auftreten kann. Die Arbeit von Moritz Roscher [179], siehe auch Kapitel 9.4.3 im Anhang, hatte die Auswirkung von gelöstem Wasserstoff auf mechanische Eigenschaften und das Bruchverhalten zum Thema.

Wird reines Eisen bei sehr hohen Stromdichten elektrolytisch mit Wasserstoff beladen, kommt es zur Bildung von Wölbungen, sog. Blistern auf der Oberfläche des Materials. Diese haben bei den Untersuchungen in der Bachelorarbeit von Annegret Lehmberg [178] Durchmesser im Bereich von 0,3 mm, vgl. Kapitel 9.4.1 im Anhang. Zur Bildung von Blistern kommt es, indem nahe unter der Oberfläche des Materials Risse entstehen, welche das Material darüber zur Oberfläche hin wegdrücken. Der Mechanismus der Entstehung verläuft wie folgt: Wasserstoff diffundiert im Eisen zu geeigneten Stellen und reichert sich dort an. Wie sich in der weiteren Erforschung von Tiegel et. al. [18] herausgestellt hat, sind dies Manganoxidausscheidungen. Wasserstoff führt hier zur Spaltung der Oberfläche zwischen Ausscheidung und Eisen. Durch die weitere Anlagerung von Leerstellen wird ein Hohlraum gebildet. Der Wasserstoff kann nun zu Wasserstoffgas rekombinieren, was zum Druckanstieg führt. Ist ein Druck im Bereich der Fließgrenze des Eisens erreicht, reißt der Hohlraum auf und es entsteht ein Riss. Dieser füllt sich erneut mit Wasserstoffgas bis wieder ein Druck im Bereich der Fließgrenze erreicht ist. Der Riss vergrößert sich in einzelnen Schritten nach und nach. Unterstützt wird dieses Modell durch die Messung des Wasserstoffdrucks in den Rissen nach der Beladung in der Arbeit von Annegret Lehmberg [178]. Es wurde ein Wasserstoffgasdruck von ca. $100 \mathrm{MPa}$ gemessen, was im Bereich der Fließgrenze von reinem Eisen von 76-97 MPa [180, 181] liegt. Die genauen thermodynamischen Hintergründe sind in [182] erläutert.

In der Arbeit von Tiegel et. al. [18] wurde die Mikrostruktur unterhalb der Rissoberfläche untersucht. Hier stellt sich heraus, dass dort eine enorme Versetzungsdichte vorliegt. Nach den Erkenntnissen der vorliegenden Arbeit bedeutet dies, dass die Bildung der Blister unter anderem möglich ist, weil Versetzungen bei Anwesenheit von Wasserstoff bei geringeren Energien gebildet werden können. So kommt es bei der Bildung der Risse zur Entstehung vieler Versetzungen, welche miteinander interagieren und so eine große Versetzungsdichte im Material zurückbleibt.

Um den Einfluss von Wasserstoff auf das Bruchverhalten von Metallen zu untersuchen, wurden in der Arbeit von Moritz Roscher [179], vgl. hierzu auch Kapitel 9.4.3 im Anhang, verschiedene Metalle mit gelöstem Wasserstoff in Zugversuchen verformt. Dabei stellt sich heraus, dass sich die Metalle Nickel, Vanadium und Tantal ohne Wasserstoff duktil verformen. Wird jedoch Wasserstoff in ihnen gelöst, kommt es zu einem scheinbaren spröden Verhalten. Die Bruchfläche ähnelt äußerlich einem spröden Bruch, jedoch ist bekannt im Falle von Nickel, dass derartige durch Was- 
serstoff hervorgerufene spröde Brüche eine sehr hohe Versetzungsdichte unterhalb der Oberfläche aufweisen. In der Arbeit von Robertson et. al. [23] wurde die Mikrostruktur unterhalb von derartigen Brüchen mit Hilfe von TEM-Untersuchungen dargestellt. Es stellt sich heraus, dass im Falle vom Nickel nicht nur unter spröden Rissen, wie sie in der Arbeit von Moritz Roscher [179] untersucht wurden, sondern auch unter intergranular (zwischen den einzelnen Körnern) gerissenen Proben eine enorme Versetzungsdichte vorliegt. Daher wird im Rahmen dieser Arbeit davon ausgegangen, dass sich bei der Verformung mit gelöstem Wasserstoff viele Versetzungen bilden. Diese interagieren miteinander, wodurch die zurückbleibende Versetzungsdichte ansteigt. Je höher die Versetzungsdichte wird, umso schwieriger ist es weitere Versetzungen hinzuzufügen. Ab einer bestimmten Versetzungsdichte ist die Bildung eines spröden Risses, um die aufgezwungene Verformung vollziehen zu können, dann energetisch günstiger.

Das spröde Verhalten bei gelöstem Wasserstoff im Falle von Vanadium und Tantal kann jedoch nicht auf die erhöhte Bildung von Versetzungen zurückgeführt werden. Basierend auf vergleichbaren Untersuchungen an Niob in der Gruppe von Birnbaum [15, 16] wird davon ausgegangen, dass beim Zugversuch durch die mechanische Spannung sich lokal die Hydridphase ausbildet. Dieses Hydrid bricht daraufhin spröde.

Diese hier vorgestellten Experimente zeigen, dass die Vorgänge der Wasserstoffversprödung auf wenigen Veränderungen auf der Ebene der Kristalldefekte beruhen. Die Vielzahl der Erscheinungen kommen durch das dynamische Zusammenspiel einzelner Defektgattungen miteinander und untereinander zustande, worin die Komplexität der Wasserstoffversprödung begründet liegt. 


\section{Zusammenfassung}

Gelöster Wasserstoff in Metallen führt in vielen Fällen zu einer Reduzierung der Güte von mechanischen Eigenschaften. Dies äußert sich auf vielfältige Weise und wird unter dem Begriff Wasserstoffversprödung zusammengefasst. Für ein grundlegendes Verständnis dieses Phänomens müssen die Vorgänge im Metall auf mikroskopischer Skala ergründet werden. Im Rahmen dieser Arbeit wurde daher ein Aspekt der Wasserstoffversprödung, die Interaktion von Wasserstoff mit Versetzungen, näher untersucht.

Zur Untersuchung des Einflusses von Wasserstoff auf die Versetzungsbildung wurden verschiedene Verformungsexperimente an Palladium und Vanadium durchgeführt. Prinzipielle Vorgänge der Defektbildung wurden durch Versuche an einzelnen Versetzungen unter Verwendung von Nanoindentation und Zugexperimenten im ETEM durchgeführt, um einen breiten Überblick zu erlangen. Zusätzlich wurden zum besseren Verständnis der Vorgänge Molekulardynamiksimulationen von derartigen Versuchen ausgeführt. Zur Untersuchung der Interaktion von Versetzungen miteinander wurden Säulen im Mikrometerbereich verformt und Blech durch Kaltwalzen verformt. Des Weiteren wurde durch Hochdruck-Torsion maximale Verformungen realisiert.

Die verwendeten Modellmaterialien erlauben es verschiedene prinzipielle Vorgänge der Defektbildung zu untersuchen und so einen breiten Überblick über prinzipielle Vorgänge im kfz Gitter (Palladium) bzw. krz Gitter (Vanadium) zu erhalten.

\section{Einfluss von gelöstem Wasserstoff auf die Defektbildung}

Anhand von Nanoindentation konnte gezeigt werden, dass Wasserstoff die Linienenergie von Versetzungen reduziert. Für diese Versuche wurde eine Spitze mit einem Radius im Nanometerbereich in eine Metalloberfläche gedrückt. Dabei wurde die dazu benötigte Kraft aufgezeichnet. Bei diesen Versuchen wurden nahezu versetzungsfreie Proben verwendet. Sowohl Palladium als auch Vanadium zeigen dabei ein ähnliches Verhalten. Aus den Kraft-Eindringkurven kann der Übergang vom elastischen zum plastischen Verhalten anhand eines Pop-Ins erkannt werden. Vanadium zeigt zwei solcher Pop-Ins. Der erste konnte dem Aufbrechen der natürlichen Oxidschicht des Vanadiums zugeordnet werden und ist unabhängig von der Wasserstoffkonzentration. Der zweite wird durch die Entstehung erster Versetzungen unterhalb der Nanoindenterspitze erklärt. Beide Metalle zeigen im Falle von gelöstem Wasserstoff, dass die Kraft, bei der der Pop-In auftritt, sinkt. Dies wird, gestützt durch das Defactant-Konzept [27, 28], damit erklärt, dass die Linienenergie von Versetzungen durch die Segregation von Wasserstoff an diesen reduziert wird.

Gestützt durch Molekulardynamiksimulationen von Nanoindentationsversuchen wird der Verlauf dieser Kraft-Eindringkurven wie folgt interpretiert: Im Anfangsbereich wird nur eine elastische Verformung durchgeführt. Wenn die ersten Versetzungen entstehen, kommt es zum Pop-In. Das bedeutet, dass ein weiteres Einfahren des Indenters nur zur Bewegung von Versetzungen führt und so keine Änderung der aufzuwendenden Kraft benötigt wird. Wenn die aufgezwungene Verformung nicht mehr durch Bewegung von Versetzungen möglich ist, müssen neue Versetzungen gebildet werden, wofür Energie benötigt wird. Somit steigt die zur Verformung benötigte Kraft wieder an und das Ende des Pop-Ins ist erreicht. Somit konnte gezeigt werden, dass der Pop-In der Übergang vom elastischen zum plastischen Verhalten ist.

Anhand von Zugversuchen im ETEM konnte gezeigt werden, dass die Lösung von Wasserstoff in Metall dazu führt, dass Versetzungen anfangen sich zu bewegen und näher aneinander rücken können. Bei Vanadium führt dies zum Wachstum eines vorhandenen Risses. Diese Beobachtungen zeigen, dass das HELP-Modell [21-23] auch auf Palladium und Vanadium anwendbar ist.

Diese Effekte werden mit Hilfe des Defactant-Konzepts [27, 28] erklärt. Gelöster Wasserstoff verzerrt das Metallgitter. Der Wasserstoff liefert also neben der Erhöhung der Entropie durch die Lösung weitere Beiträge zur Freien Energie des Metalls. Eine Stufenversetzung in Palladium und Vanadium verzerrt das Gitter, sodass größere Zwischengitterplätze entstehen. Diffundiert Wasserstoff zu diesen Plätzen, reduziert dies die Verzerrung des Gitters und somit die freie Energie des Metalls. Die Interpretation des Defactant-Konzepts ist, dass eine Versetzung ihre Energie reduziert, indem Wasserstoff an ihr segregiert. 


\section{Erhöhung der Versetzungsdichte durch Wasserstoff}

Alle weiteren Experimente in dieser Arbeit beruhen auf der Erkenntnis, dass Wasserstoff die Linienenergie von Versetzungen reduziert. Dies bedeutet, dass bei gleichem Verformungsgrad mehr Versetzungen gebildet werden können.

Um diesen Effekt nachvollziehen zu können, wurden zwei weitere Versuchsreihen durchgeführt. Anhand von Druckversuchen an Vanadiumsäulen konnte der Effekt indirekt nachgewiesen werden. Es wurden Säulen im Mikrometerbereich in die Oberfläche eines Einkristalls geschnitten. Enthalten diese Säulen keinen oder nur wenig Wasserstoff bei der Verformung, gleiten die Säulen auf wenigen Ebenen ab. Bei höheren Wasserstoffkonzentrationen sind kaum noch diskrete Abgleitebenen zu erkennen und die Säulen nehmen die Form eines Fasses an. Auf der Oberfläche der wasserstoffreichen Säulen sind nach der Verformung feine Linien zu erkennen, welche als minimale Abgleitungen interpretiert werden. Zudem widerstehen die Säulen größeren Kräften. Diese zunächst widersprüchlich erscheinenden Ergebnisse werden damit erklärt, dass wenn Wasserstoff im Metall gelöst ist, mehr Versetzungen gebildet werden und diese nicht rechtzeitig wieder aus dem Material heraus wandern können. Somit müssen die Versetzungen miteinander interagieren, was dazu führt, dass eine hohe Versetzungsdichte in der Säule verbleibt, wodurch eine Verformung erschwert wird.

Ein direkter Nachweis der Erhöhung der Versetzungsdichte durch Wasserstoff wurde anhand von kaltgewalztem Palladium erbracht. Palladium wurde hierzu mit verschiedenen Wasserstoffkonzentrationen beladen und gewalzt. TEM-Aufnahmen dieser Proben zeigen, dass die Versetzungsdichte deutlich mit der Wasserstoffkonzentration zunimmt. Ohne Wasserstoff bilden sich große Versetzungszellen aus. Durch die Hinzugabe von 0,001 H/Pd entstehen unregelmäßige dichtere Versetzungsanhäufungen. Bei weiterer Erhöhung bilden sich erneut Versetzungszellen, welche bei $0,01 \mathrm{H} / \mathrm{Pd}$ so dichte Zellwände haben, dass von Subkörnern gesprochen werden kann. Weitere Experimente bei denen Palladium durch HPT verformt wurde, zeigen, dass sich dieser Trend fortsetzt. Bei höheren Verformungsraten entstehen nanokristalline Körner.

Damit lassen sich die Auswirkungen von Wasserstoff auf Versetzungen wie folgt zusammenfassen: Gelöster Wasserstoff führt zur Reduzierung der Energie, die zur Bildung von Versetzungen nötig ist. Dadurch werden bei gleicher Verformung mehr Versetzungen gebildet. Wasserstoff bewirkt auch eine Reduzierung der Interaktionsenergie zwischen Versetzungen. Dies führt zu erhöhter Beweglichkeit von Versetzungen, wodurch diese besser einem äußeren mechanischen Spannungsfeld folgen können. Eine Auswirkung von diesem Verhalten ist z. B. die Erleichterung des Wachstums von Rissen. Dies zeigt, dass die Materialauswahl bei der Konstruktion von OffshoreWindenergieanlagen, welche dem korrosiven Meerwasser ausgesetzt sind, von entscheidender Bedeutung für eine lange Nutzungsdauer ist. 


\section{Ausblick}

Im Rahmen dieser Arbeit wurde unter anderem die Dynamik des Zusammenspiels von Wasserstoff mit wenigen Versetzungen auf mikroskopischer Skala untersucht. Die Analyse makroskopischer Erscheinungen der Wasserstoffversprödung bei Verformungsexperimenten anhand mikroskopischer Vorgänge stellt eine große Herausforderung dar. Durch die Aufnahme von mechanischen Parametern während der Verformung können nur indirekte Aussagen über die Defektentwicklung getroffen werden.

Eine andere Möglichkeit ist die Untersuchung der Defektstruktur im Nachhinein bspw. im TEM. Um die Dynamik der Versetzungsentstehung zu verstehen, wäre z. B. eine Methode zur in-situ Bestimmung der Versetzungsdichte bei Zugversuchen hilfreich. Eine Möglichkeit dies zu bewerkstelligen ist es, Wasserstoff als Sonde zu verwenden. Um die relative Zunahme der Versetzungsgesamtlänge während eines Zugversuchs zu bestimmen, kann eine ähnliche Herangehensweise wie bei der Methode, welche in der Diskussion vorgestellt und in der Veröffentlichung [40] nachvollzogen werden kann, verwendet werden. Im Anhang in Kapitel 9.1 werden zwei mögliche Verfahrensweisen vorgestellt. Bei diesen Methoden wird eine mit Wasserstoff beladene Probe innerhalb eines Elektrolyten einem Zugversuch ausgesetzt. Gleichzeitig wird die elektromotorische Kraft (EMK) gemessen. Die EMK hängt vom frei beweglichen Wasserstoff in der Probe ab. Bei der Bildung von Versetzungen wird Wasserstoff an Versetzungen gebunden und trägt somit weniger zur EMK bei. Dies wird ausgenutzt, um die Veränderung der Versetzungsdichte während des Zugversuchs $\mathrm{zu}$ ermitteln.

In zukünftigen Arbeiten können diese Methoden verwendet werden, um entweder die relative Änderung der Versetzungsgesamtlänge bezogen auf eine konstante Wasserstoffkonzentration oder auf ein konstantes chemisches Potential zu bestimmen. Beide Methoden spiegeln Situationen, welche in der Realität auftreten, wider. Die erste Methode zeigt den Effekt von Wasserstoff auf eine im Bezug zum verformten Bereich kleine Probe. Ein Beispiel ist eine unter Zugspannung stehende Schraube. Die andere Methode zeigt den Effekt auf eine große Probe, die es ermöglicht Wasserstoff zum verspannten Bereich nachzuliefern. Als Beispiel sei eine Schweißnaht genannt. 


\section{Anhang}

\subsection{Möglichkeiten zur Protokollierung der Versetzungsentwicklung während Zugversuchen}

Im Rahmen der Arbeit wurde die Dynamik des Zusammenspiels von Wasserstoff mit wenigen Versetzungen auf mikroskopischer Skala untersucht. Um die Versetzungsdynamik auch auf makroskopischer Ebene direkt untersuchen zu können, muss ein Weg gefunden werden Versetzungen sichtbar zu machen. In der Veröffentlichung [40] wurden zwei prinzipielle Methoden aufgezeigt. Einerseits ist es möglich mit der Auswertung von Röntgenbeugungsdiagrammen die absolute Versetzungsdichte zu bestimmen. Andererseits kann Wasserstoff als Sensor für Versetzungen genutzt werden.

Im Folgenden wird beschrieben, wie ausgehend von den theoretischen Grundlagen [40] die Versetzungsdichte in-situ bei Zugversuchen bestimmt werden kann. Der in der betreuten Bachelorarbeit von Moritz Roscher [179] entwickelte in-situ Zugaufbau eignet sich hervorragend um die Dynamik der Versetzungsbildung bei Zugversuchen zu untersuchen. Dieser Aufbau, vgl. Kapitel 9.4.3, ermöglicht die Messung der elektromotorischen Kraft (EMK) während des Zugversuchs. Wie in der Diskussion angedeutet und in der Veröffentlichung [40] beschrieben, kann aus einer Veränderung der EMK die (relative) Versetzungsdichte bestimmt werden. Bei einem Zugversuch bildet sich die Versetzungsdichte in der Regel nicht homogen aus. Wird das gleiche Verfahren angewendet, wie in der Veröffentlichung [40] beschrieben, wird daher der relative Unterschied in der Gesamtlänge aller Versetzungen bestimmt. Die Verwendung eines in-situ Zugversuchs ermöglicht somit während des Zugversuchs einer Probe die Entstehung von Versetzungen zu verfolgen.

Der Aufbau in Abbildung 78 in Kapitel 9.4.3 besteht aus einem Plexiglasgefäß, welches an der unteren Probenfassung angebracht wurde. In dieses Gefäß kann eine $\mathrm{Ag} / \mathrm{AgCl}$ Bezugselektrode eingebracht werden, um die elektromotorische Kraft (EMK), welche proportional zum Logarithmus des chemischen Potentials $\left(\mu_{\mathrm{H}}\right)$ des Wasserstoffs in der Probe ist, zu bestimmen.

Die Messung der EMK während des Zugversuchs eröffnet ein breites Spektrum an experimentellen Möglichkeiten.

Die EMK $U$ kann nach der Nernstschen Gleichung dargestellt werden:

$$
\begin{aligned}
U & =U_{0}+\frac{R T}{F} \ln \left(a_{H}\right) \\
& =U_{0}+\frac{R T}{F} \ln \left(\gamma^{*} c_{H}\right)
\end{aligned}
$$

mit $R$ der universellen Gaskonstanten, $T$ der Temperatur, $F$ der Faradaykonstante und $a_{H}$ der Aktivität des Wasserstoffs in der Probe. Die Aktivität kann über den Aktivitätskoeffizienten $\gamma^{*}$ mit der Wasserstoffkonzentration $c_{H}$ verknüpft werden.

Der Aktivitätskoeffizient $\gamma^{*}$ beschreibt, wie viel Wasserstoff zur EMK beiträgt. Wird der Wasserstoff an Kristalldefekten gebunden, trägt dieser weniger zur EMK bei [61, 183-185] und zwar umso weniger je fester der Wasserstoff gebunden ist [185]. Dies bedeutet auch, dass wenn die Atomabstände im elastischen Bereich eines Zugexperiments vergrößert werden, Wasserstoff durch die größer werdenden Zwischengitterplätze fester gebunden wird. Dies wurde bei Zugversuchen an einem Palladiumdraht von Kirchheim [82] gezeigt. Hierbei ergibt sich für die Änderung der EMK $(\Delta U)$ mit der Zugbelastung $\sigma$ :

$$
\Delta U=\frac{V_{H}}{3 F} \sigma,
$$

mit $V_{H}$ dem partiellen molaren Volumen des Wasserstoffs. 
Die Veränderungen der EMK durch elastische Dehnung und durch plastische Verformung können auseinander gehalten werden, da die Bildung von Versetzungen zur permanenten Änderung der EMK führt und der Beitrag durch die elastische Verformung wieder verschwindet, wenn die Belastung zurückgenommen wird [82].

Ziel der folgenden Überlegungen ist es einen Ausdruck für den Aktivitätskoeffizient $\gamma^{*}$ zu erhalten. Unter der Annahme, dass sich die plastische Dehnung im Zugversuch nur durch die Bildung von Versetzungen ergibt, kann aus der Änderung des Aktivitätskoeffizienten $\gamma^{*}$ die Versetzungsdichte bestimmt werden. Dabei wird analog zu den Experimenten an gewalztem Palladium [40] vorgegangen.

\section{Bestimmung der Versetzungsdichte während Zugexperimenten bei fester Wasserstoffkonzentration}

Wird die EMK bei einem Zugversuch bei fester Konzentration gemessen, steigt diese im elastischen Bereich zunächst nach Gleichung 9.3. Im plastischen Bereich wird der Beitrag von Gleichung 9.3 konstant, jedoch werden nun Versetzungen gebildet, d. h. Wasserstoff wird an Versetzungen gebunden und der Aktivitätskoeffizienten $\gamma^{*}$ sinkt und damit sinkt auch die EMK.

Wird die EMK gegen die (wahre ${ }^{33}$ ) Spannung aufgetragen, sollte sich zunächst der erwartete lineare Verlauf ergeben. Bei Erreichen der Fließgrenze, fällt die Kurve. Der Unterschied zwischen dem verlängerten linearen Verlauf und dem tatsächlichen Verlauf $\left(\Delta U=U_{\text {elastisch }}-U_{\text {plastisch }}\right)$ ergibt sich zu:

$$
\begin{aligned}
U_{\text {elastisch }} & =U_{0} \frac{R T}{F} \ln \left(\gamma_{0}^{*} c_{H}\right)+\frac{V_{H}}{3 F} \sigma \\
\Delta U=U_{\text {elastisch }}-U_{\text {plastisch }} & =\frac{R T}{F} \ln \left(\frac{\gamma_{\text {plastisch }}^{*}}{\gamma_{0}^{*}}\right)+\frac{V_{H}}{3 F} \sigma
\end{aligned}
$$

Für kleine Konzentrationen und bei vernachlässigbarer Versetzungsdichte gilt $\gamma_{0}^{*}=1$ und somit folgt:

$$
\gamma_{\text {plastisch }}^{*}=\exp \left(\frac{F}{R T}\left(\Delta U-\frac{V_{H}}{F} \sigma\right)\right)
$$

Der gebundene Wasserstoff $c_{t}$ ergibt sich nach [40]:

$$
c_{t}=c_{H}\left(1-\gamma_{\text {plastisch }}^{*}\right)
$$

Des Weiteren ergibt sich $c_{t}$ durch den Unterschied des gelösten Wasserstoffs einer ausgelagerten Probe $\left(\frac{n_{\mathrm{H}}}{n_{\mathrm{M}}}\right)_{\text {ausgelagert }} \mathrm{zu}$ dem einer verformten Probe $\left(\frac{n_{\mathrm{H}}}{n_{\mathrm{M}}}\right)_{\text {verformt }}$ bei gleichem chemischen Potential $\mu_{\mathrm{H}}[28]$ :

$$
c_{t}=\left[\left(\frac{n_{\mathrm{H}}}{n_{\mathrm{M}}}\right)_{\text {verformt }}-\left(\frac{n_{\mathrm{H}}}{n_{\mathrm{M}}}\right)_{\text {ausgelagert }}\right]=\rho \Gamma_{\mathrm{H}}\left(\mu_{\mathrm{H}}\right) \Omega_{\mathrm{M}}
$$

Der fest gebundene Wasserstoff hängt von der Versetzungsdichte $\rho$, dem molaren Volumen $\Omega_{\mathrm{M}}$ des Metalls und dem Excess $\Gamma_{\mathrm{H}}\left(\mu_{\mathrm{H}}\right)$ ab. Der Excess gibt an, wie viele Wasserstoffatome pro Burgersvektor an einer Versetzung gebunden werden können. Dieser Parameter ist unabhängig von der Versetzungsdichte, hängt jedoch vom chemischen Potential ab.

\footnotetext{
${ }^{33}$ Der Probenquerschnitt, welcher zur Bestimmung der wahren Spannung benötigt wird, kann durch die angebrachte Kamera bestimmt werden.
} 
Dies bedeutet, dass wenn zwei Proben $(A, B)$ mit verschiedenen Wasserstoffkonzentrationen gezogen werden, ein Vergleich bei gleichem chemischen Potential, d. h. bei gleicher EMK, den relativen Unterschied in der Versetzungsdichte liefert:

$$
\begin{aligned}
\frac{c_{t, A}}{c_{t, B}}(U) & =\frac{\rho_{A} \Gamma_{\mathrm{H}}\left(\mu_{\mathrm{H}}\right) \Omega_{\mathrm{M}}}{\rho_{B} \Gamma_{\mathrm{H}}\left(\mu_{\mathrm{H}}\right) \Omega_{\mathrm{M}}}=\frac{\rho_{A}}{\rho_{B}} \\
& =\frac{\rho_{A}}{\rho_{B}}(U)
\end{aligned}
$$

Das Problem dieser Methode ist, dass sich das gleiche chemische Potential im Regelfall nicht bei der gleichen Zugspannung ergibt. Eine mögliche Aussage ist bspw. bei welcher Zugspannung die gleiche Versetzungsdichte erreicht ist.

\section{Bestimmung der Versetzungsdichte während Zugexperimenten bei festem chemischen Potential}

Eine erweiterte Möglichkeit ergibt sich bei der Verwendung eines Potentiostaten. Dieser misst die EMK und versucht durch das Anlegen eines Stromes zwischen Probe und Anode die EMK konstant zu halten. Es wird also je nach Bedarf Wasserstoff in der Probe gelöst oder aus ihr entfernt. Wird beim Zugexperiment Wasserstoff an Versetzungen gebunden, trägt dieser nicht mehr dermaßen zur EMK bei, und muss daher durch neuen Wasserstoff ersetzt werden, um die EMK konstant zu halten. Die gesamt geflossene Ladung $Q=\int_{t} I(t) \mathrm{d} t$ entspricht also dem gebundenen Wasserstoff $c_{t}$. Über das Faradaysche Gesetz kann dieser bestimmt werden:

$$
\Delta c=c_{t}=\frac{\int_{t} I(t) \mathrm{d} t}{F} \frac{1}{n_{M}},
$$

mit $F$ der Faradaykonstante und $n_{M}$ der Stoffmenge der Probe.

Experimentell wird bei dieser Methode die mechanische Spannung $\sigma$ stufenweise erhöht und wieder entlastet. Im elastischen Bereich wird während der Belastung neuer Wasserstoff gelöst, der beim Entlasten wieder heraus gelöst wird.

Im plastischen Bereich werden nun Versetzungen gebildet, an denen sich Wasserstoff fester bindet. Bei Entlastung bleibt dieser Wasserstoff in der Probe zurück.

Bei der Bestimmung der Versetzungsdichte ergibt sich wieder das Problem, dass der Excess $\Gamma_{\mathrm{H}}\left(\mu_{\mathrm{H}}\right)$ unbekannt ist. Es kann daher wieder nur eine relative Versetzungsdichte bestimmt werden. Jedoch wird hier nur eine Probe benötigt. Durch Vergleich der Menge des gebundenen Wasserstoffs bei verschiedenen mechanischen Spannungen kann so der Unterschied zwischen den einzelnen (mechanischen) Spannungsstufen ermittelt werden, vgl. Gleichung 9.9.

Die Aussage dieser Methode ist wie sich die Versetzungsdichte während des Versuches erhöht. Werden verschiedene Proben mit unterschiedlichen Ausgangspotentialen miteinander verglichen, kann der Einfluss von Wasserstoff auf die Bildung von Versetzungen während eines Zugversuchs ermittelt werden. 


\subsection{Weitere Messergebnisse}

\subsubsection{Untersuchungen zur Defektbildung in Palladium-Wasserstofflegierungen durch Walzen}

In diesem Abschnitt sind weitere TEM Aufnahmen von Palladiumproben, welche durch Kaltwalzen verformt wurden, dargestellt. Genaue Versuchs- und Ergebnissbeschreibung können den Kapiteln 4.5 bzw. 5.2.2 entnommen werden.
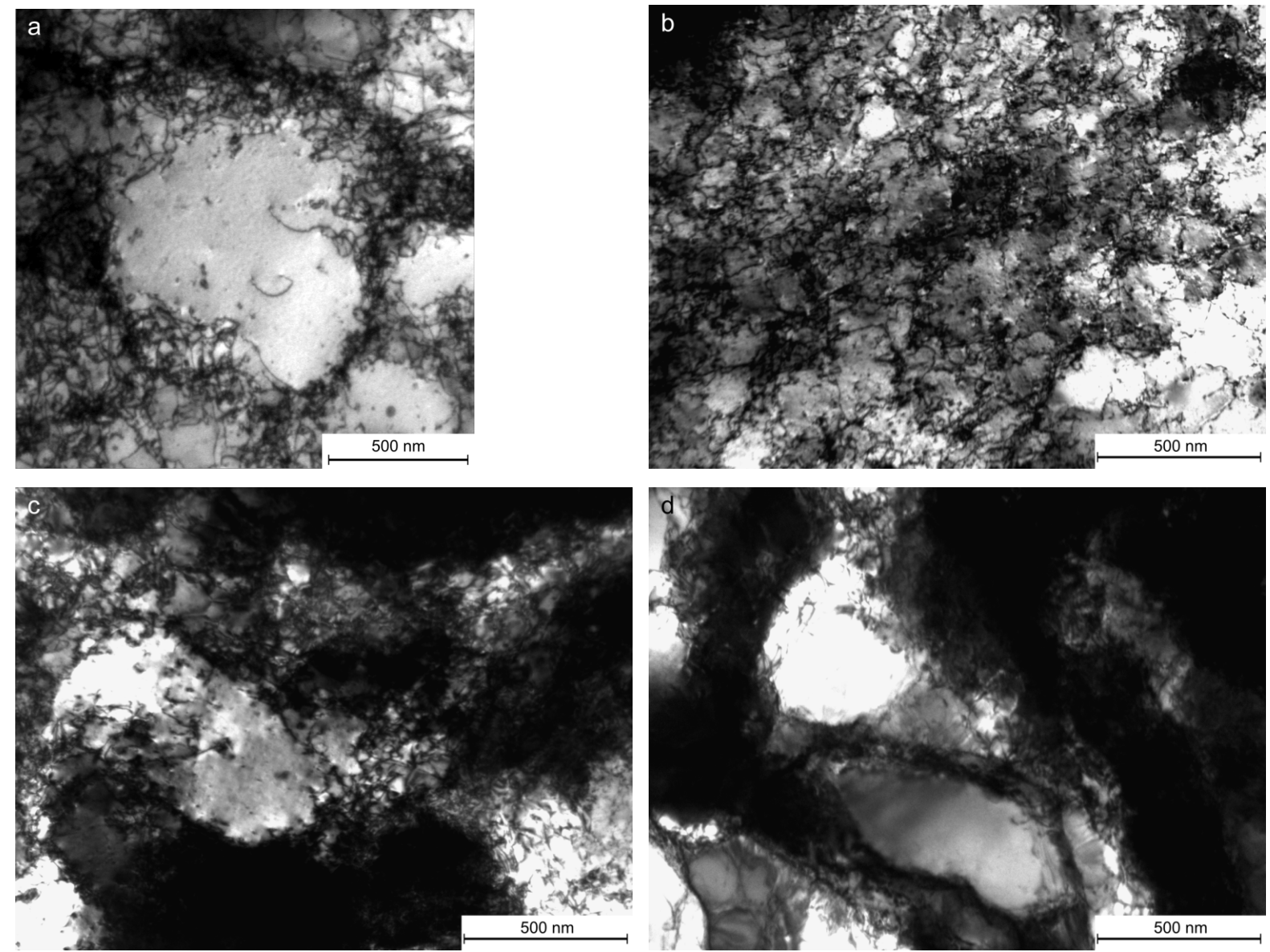

Abb. 51 TEM-Aufnahmen von kaltgewalzten Palladium-Wasserstofflegierungen[40], a zeigt eine Probe, welche ohne Wasserstoff gewalzt wurde (Untersuchung durchgeführt an einem anderen TEM), die Abbildungen b-d zeigen TEM-Aufnahmen von kaltgewalzten Palladiumblechen, welche eine Wasserstoffkonzentration von $0.1 \% \mathrm{H} / \mathrm{Pd}, 0.5 \% \mathrm{H} / \mathrm{Pd}$ bzw. $1.0 \% \mathrm{H} / \mathrm{Pd}$ bei der Verformung hatten. 


\subsubsection{Untersuchungen zur Defektbildung in Palladium-Wasserstofflegierungen bei extremer Verformung}

In diesem Abschnitt werden TEM Aufnahmen von Palladiumproben gezeigt, welche mittels High Pressure Torsions (HPT) verformt wurden. Genaue Versuchs- und Ergebnisbeschreibung können den Kapiteln 4.6 bzw. 5.2.3 entnommen werden.
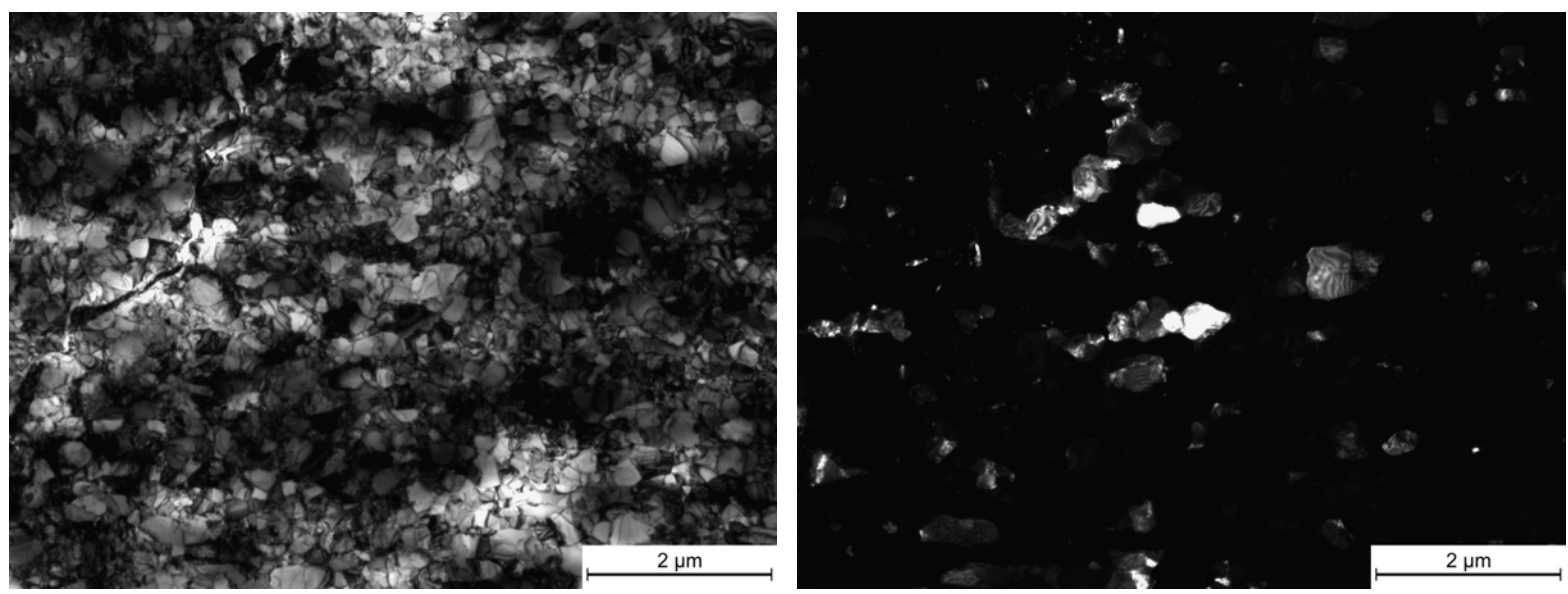

Abb. 52 Übersichtsaufnahmen einer mit 0,25\%H/Pd beladenen Palladiumprobe, welche mittels HPT bei Raumtemperatur verformt wurde. Links Hellfeldaufnahme, rechts Dunkelfeldaufnahme des gleichen Bereichs.
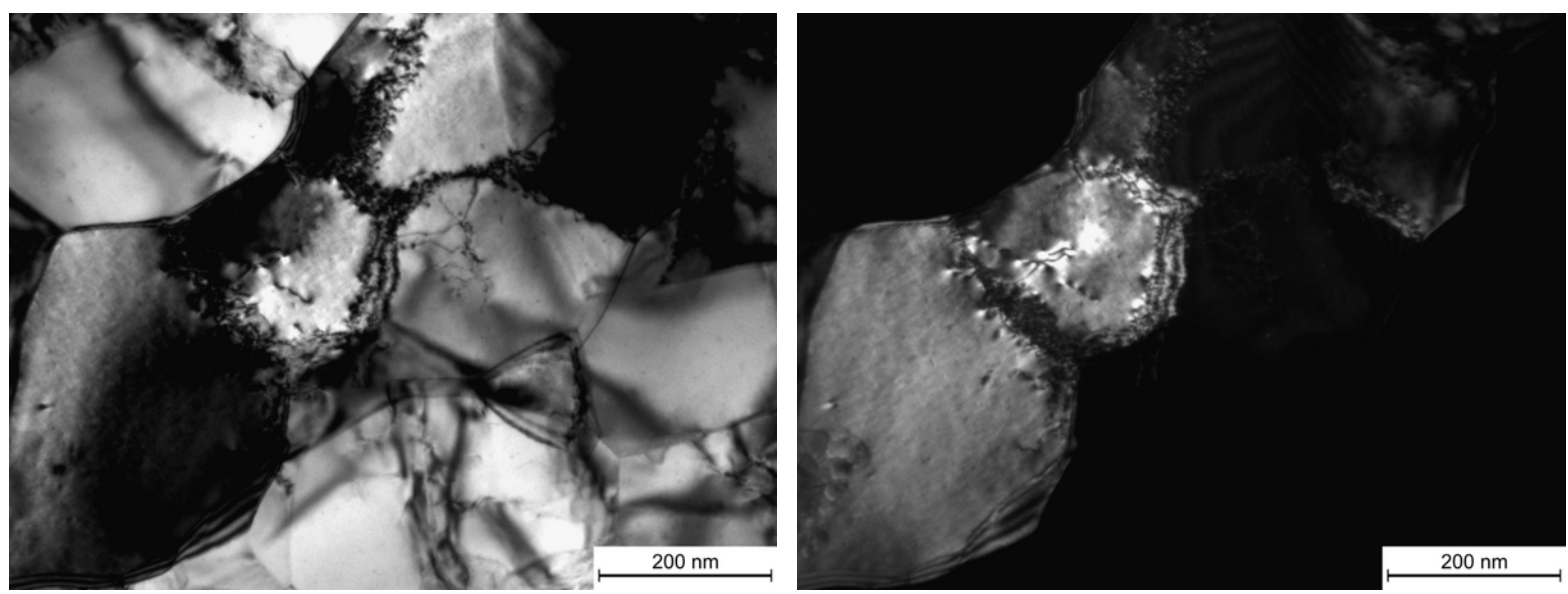

Abb. 53 TEM Aufnahmen einer mit 0,25\%H/Pd beladenen Palladiumprobe, welche mittels HPT bei Raumtemperatur verformt wurde. Links oben Hellfeldaufnahme, rechts oben Dunkelfeldaufnahme des gleichen Bereichs. Für diese wurde mit der Kontrastblende ein Bereich mit den beiden innersten Ringen im Beugungsbild (unten, Graustufen invertiert) ausgewählt. 

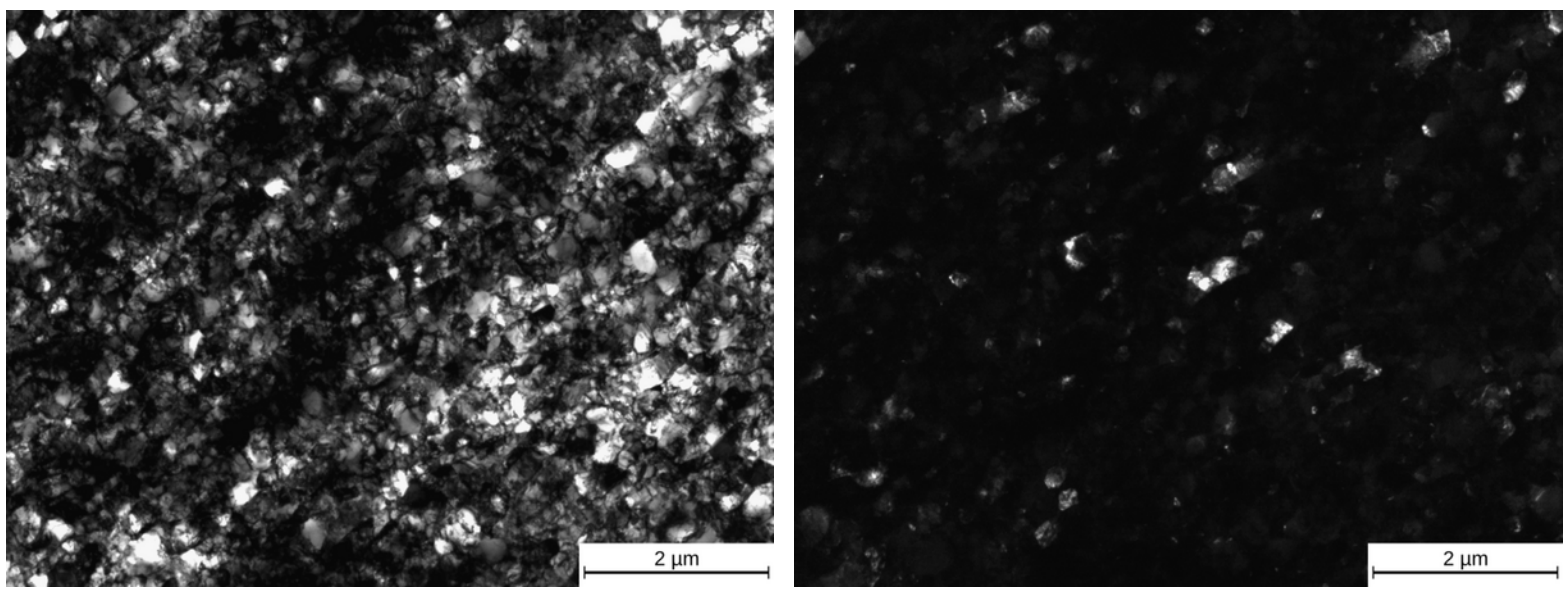

Abb. 54 Übersichtsaufnahmen einer mit 0,25\% H/Pd beladenen Palladiumprobe, welche mittels $\mathrm{HPT}$ mit $\mathrm{CO}_{2}$-Kühlung $\left(\mathrm{T} \approx-60^{\circ} \mathrm{C}\right)$ verformt wurde. Links Hellfeldaufnahme, rechts Dunkelfeldaufnahme des gleichen Bereichs.
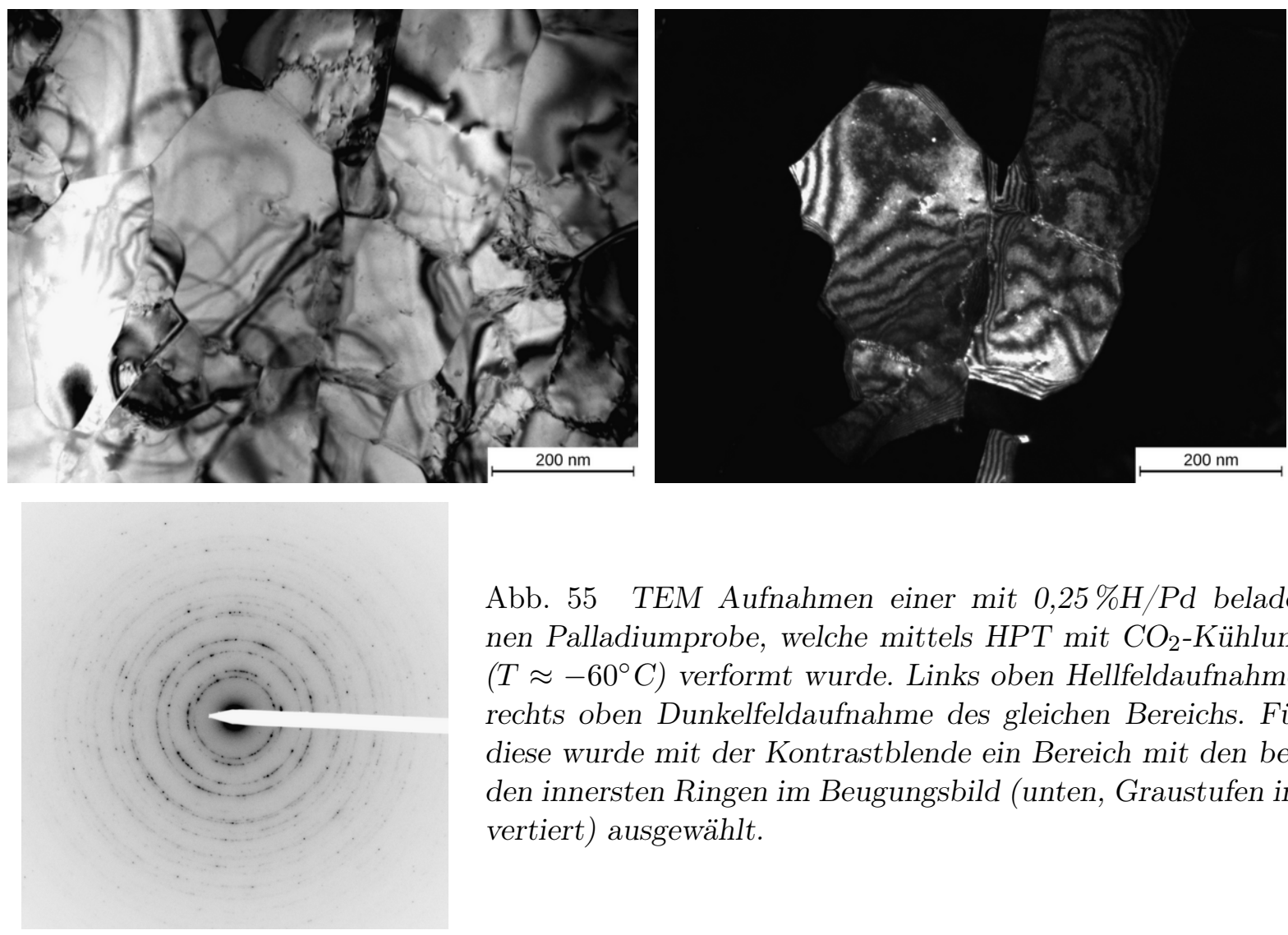

Abb. 55 TEM Aufnahmen einer mit $0,25 \% \mathrm{H} / \mathrm{Pd}$ beladenen Palladiumprobe, welche mittels $\mathrm{HPT}$ mit $\mathrm{CO}_{2}$-Kühlung $\left(T \approx-60^{\circ} \mathrm{C}\right)$ verformt wurde. Links oben Hellfeldaufnahme, rechts oben Dunkelfeldaufnahme des gleichen Bereichs. Für diese wurde mit der Kontrastblende ein Bereich mit den beiden innersten Ringen im Beugungsbild (unten, Graustufen invertiert) ausgewählt. 

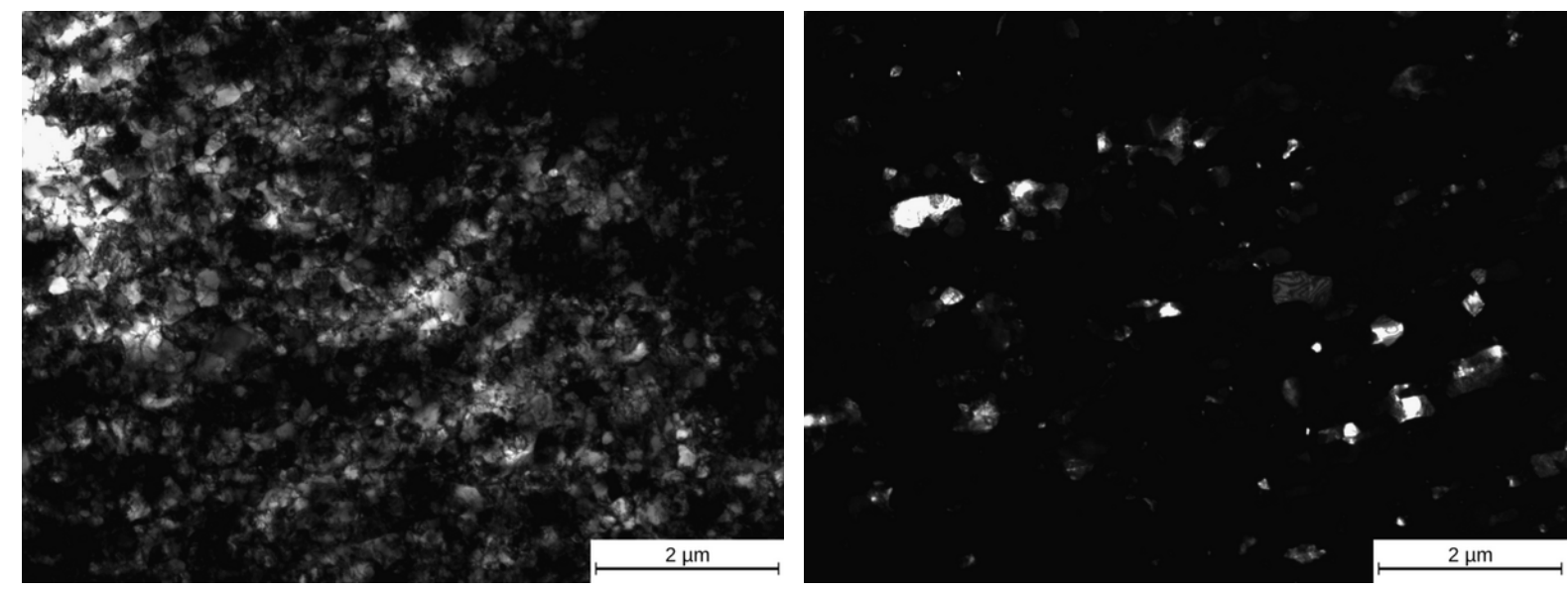

Abb. 56 Übersichtsaufnahmen einer mit 0,75\% H/Pd beladenen Palladiumprobe, welche mittels HPT bei Raumtemperatur verformt wurde. Links Hellfeldaufnahme, rechts Dunkelfeldaufnahme.

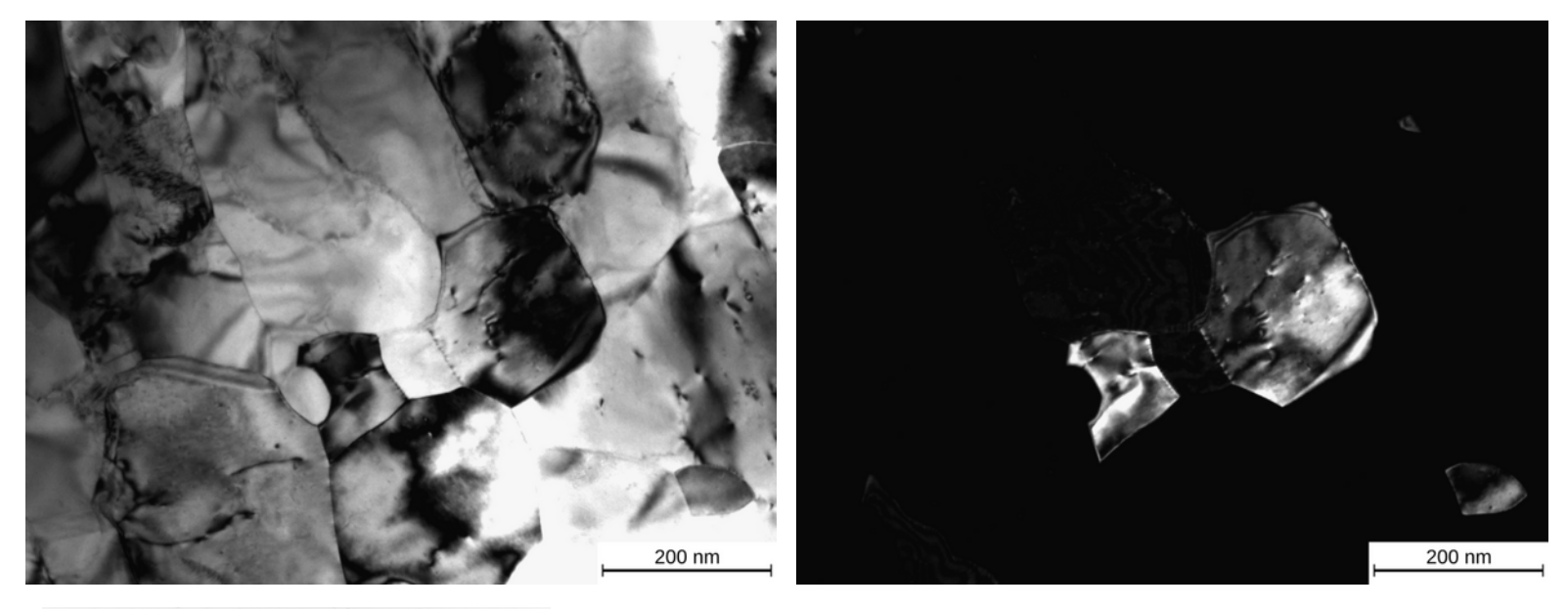

Abb. 57 TEM Aufnahmen einer mit 0,75\% H/Pd beladenen Palladiumprobe, welche mittels HPT bei Raumtemperatur verformt wurde. Links oben Hellfeldaufnahme, rechts oben Dunkelfeldaufnahme des gleichen Bereichs. Für diese wurde mit der Kontrastblende ein Bereich mit den beiden innersten Ringen im Beugungsbild (unten, Graustufen invertiert) ausgewählt. 

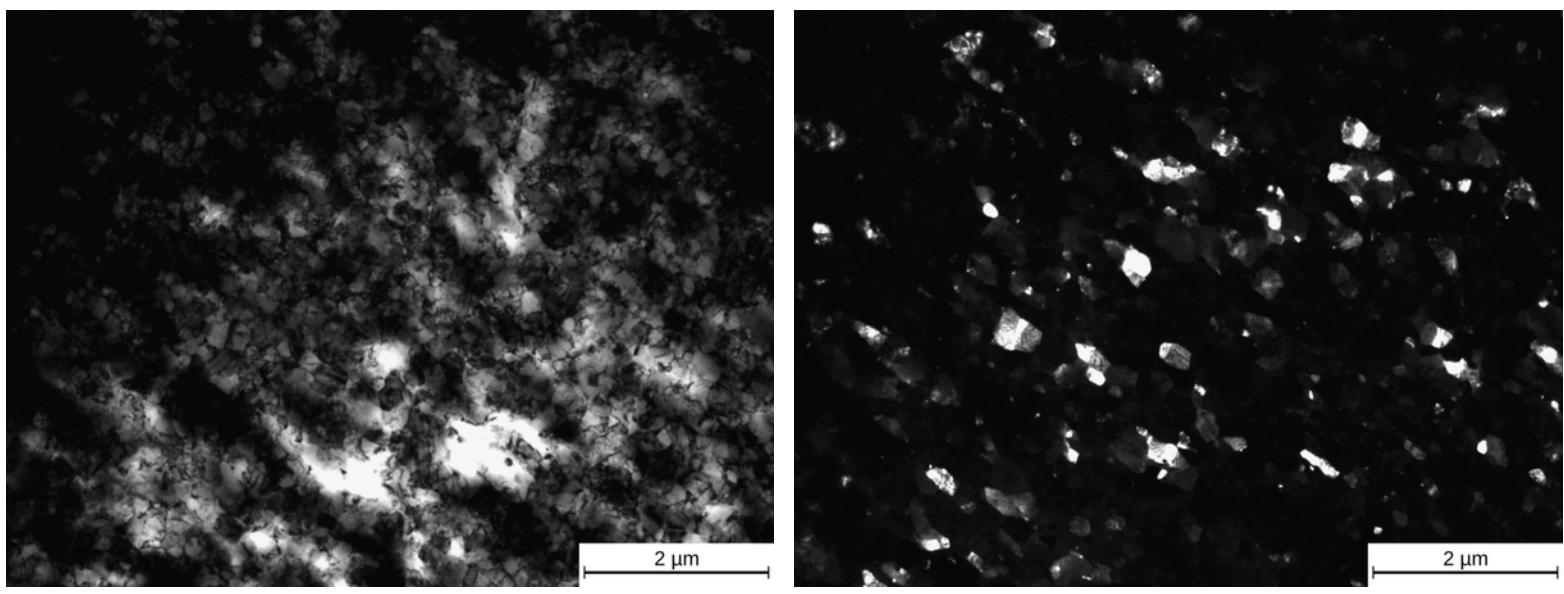

Abb. 58 Übersichtsaufnahmen einer mit 0,75\% H/Pd beladenen Palladiumprobe, welche mittels $\mathrm{HPT}$ mit $\mathrm{CO}_{2}$-Kühlung $\left(\mathrm{T} \approx-60^{\circ} \mathrm{C}\right)$ verformt wurde. Links Hellfeldaufnahme, rechts Dunkelfeldaufnahme des gleichen Bereichs.
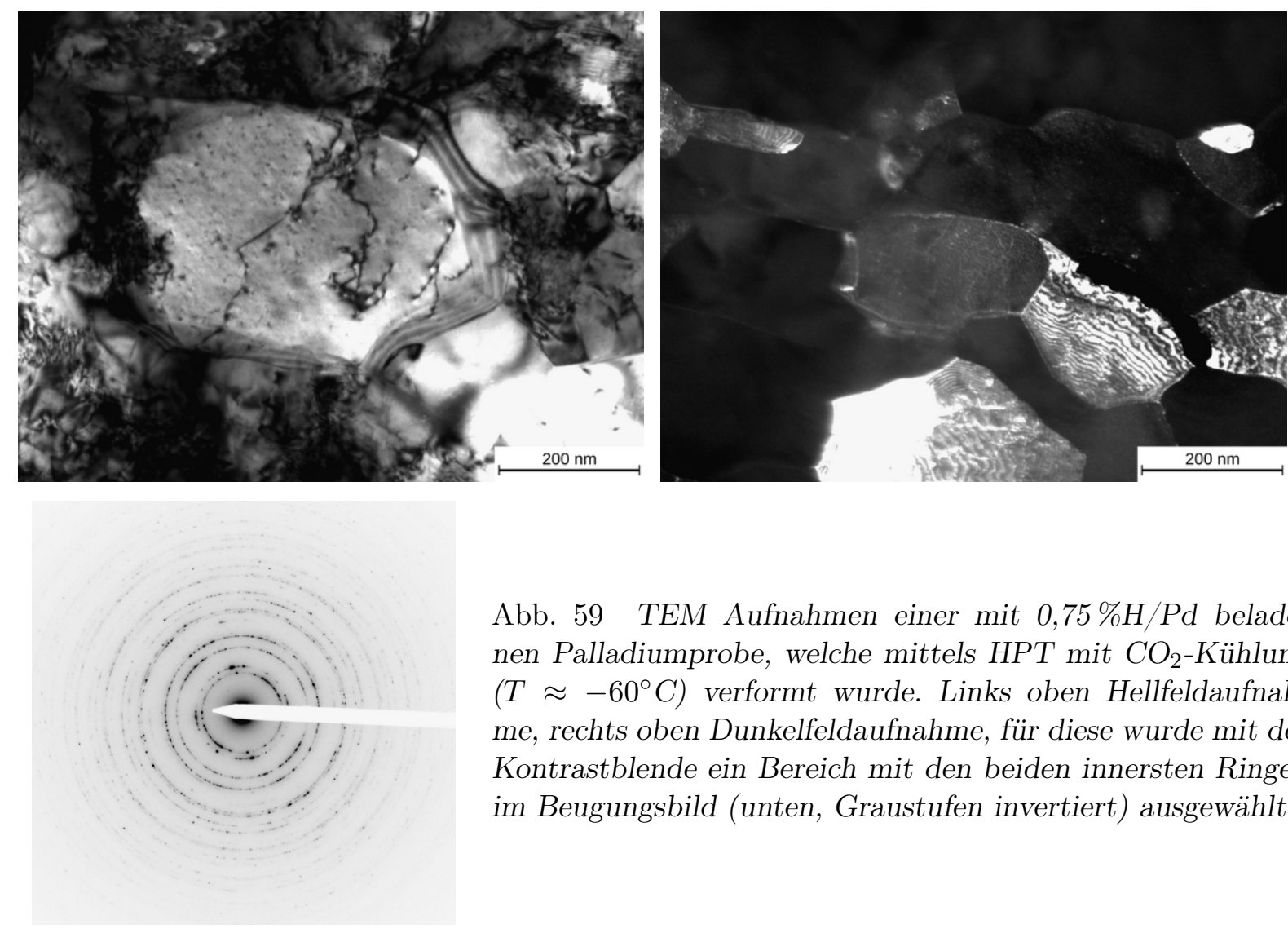

Abb. 59 TEM Aufnahmen einer mit 0,75\% $\mathrm{H} / \mathrm{Pd}$ beladenen Palladiumprobe, welche mittels $\mathrm{HPT}$ mit $\mathrm{CO}_{2}$-Kühlung $\left(T \approx-60^{\circ} C\right)$ verformt wurde. Links oben Hellfeldaufnahme, rechts oben Dunkelfeldaufnahme, für diese wurde mit der Kontrastblende ein Bereich mit den beiden innersten Ringen im Beugungsbild (unten, Graustufen invertiert) ausgewählt. 

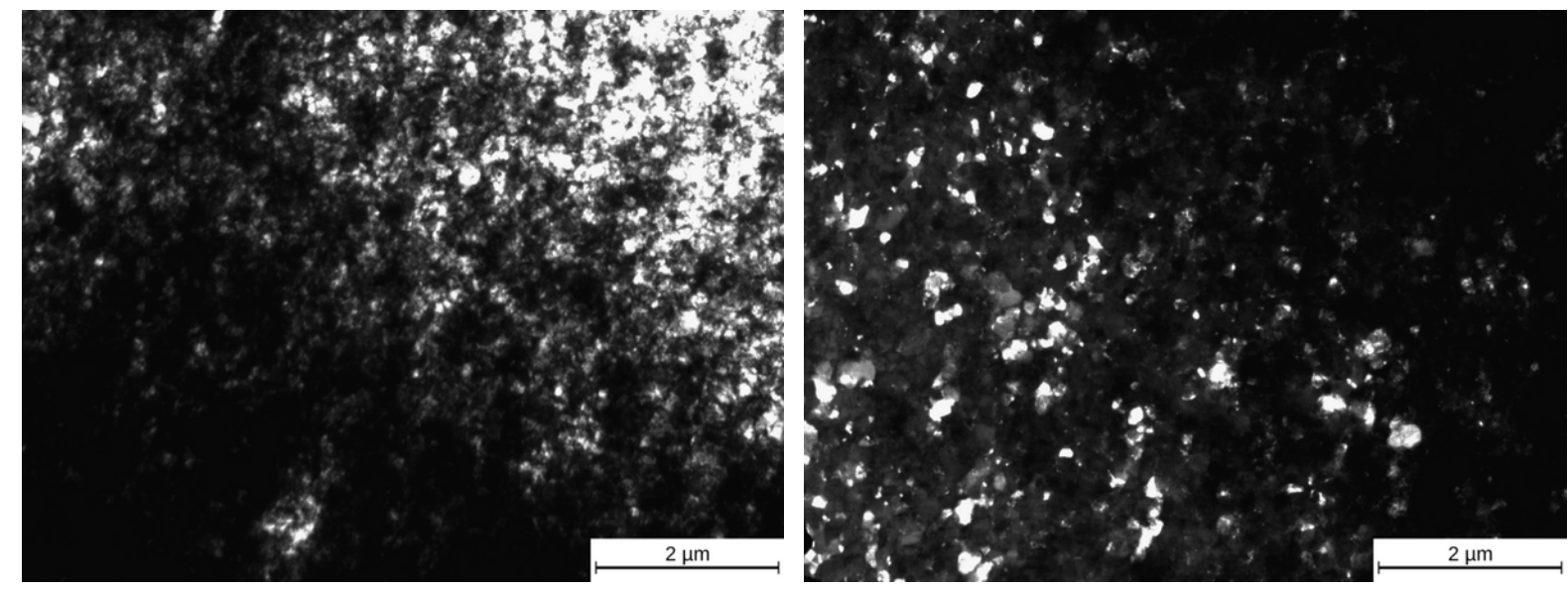

Abb. 60 Übersichtsaufnahmen einer mit 20\%H/Pd beladenen Palladiumprobe, welche mittels HPT bei Raumtemperatur verformt wurde. Links Hellfeldaufnahme, rechts Dunkelfeldaufnahme des gleichen Bereichs.
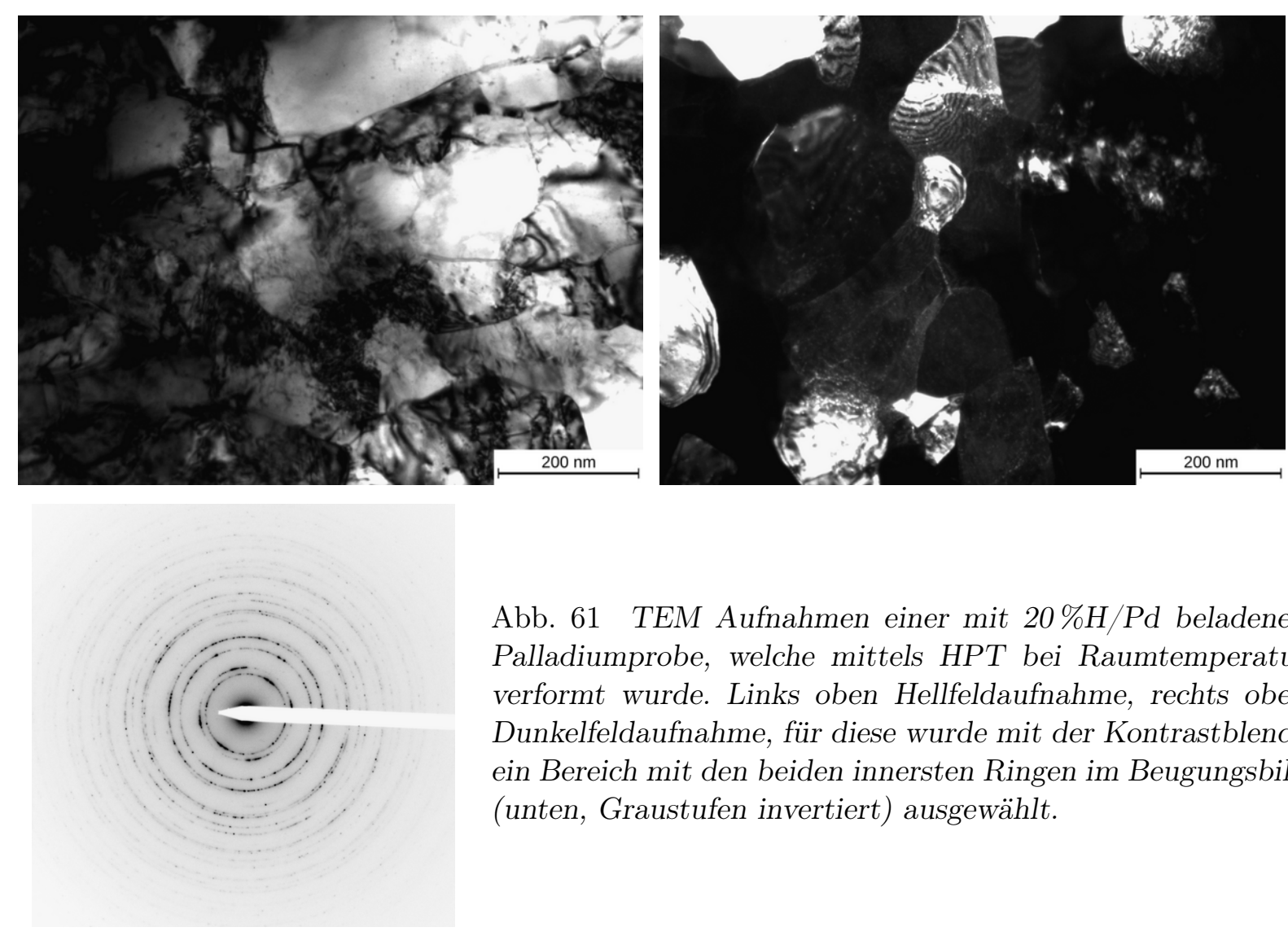

Abb. 61 TEM Aufnahmen einer mit 20\%H/Pd beladenen Palladiumprobe, welche mittels HPT bei Raumtemperatur verformt wurde. Links oben Hellfeldaufnahme, rechts oben Dunkelfeldaufnahme, für diese wurde mit der Kontrastblende ein Bereich mit den beiden innersten Ringen im Beugungsbild (unten, Graustufen invertiert) ausgewählt. 

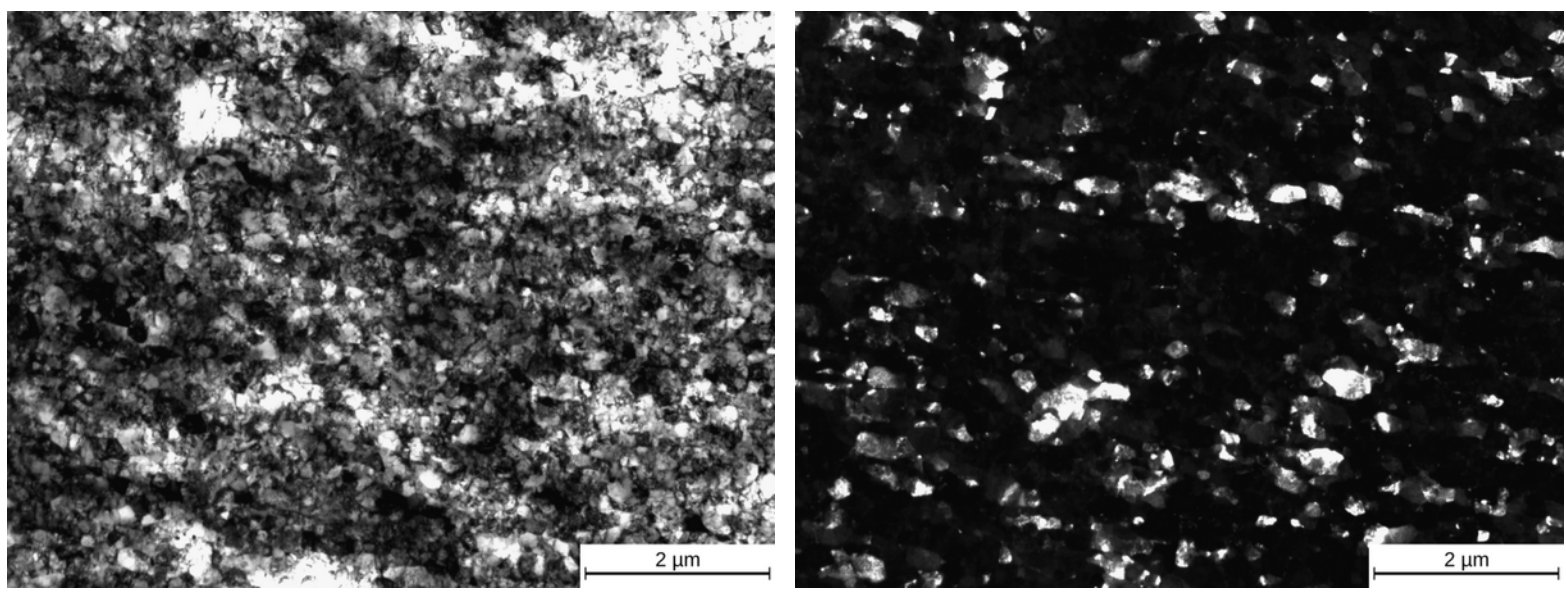

Abb. 62 Übersichtsaufnahmen einer mit 20\%H/Pd beladenen Palladiumprobe, welche mittels $\mathrm{HPT}$ mit $\mathrm{CO}_{2}$-Kühlung $\left(\mathrm{T} \approx-60^{\circ} \mathrm{C}\right)$ verformt wurde. Links Hellfeldaufnahme, rechts Dunkelfeldaufnahme des gleichen Bereichs.
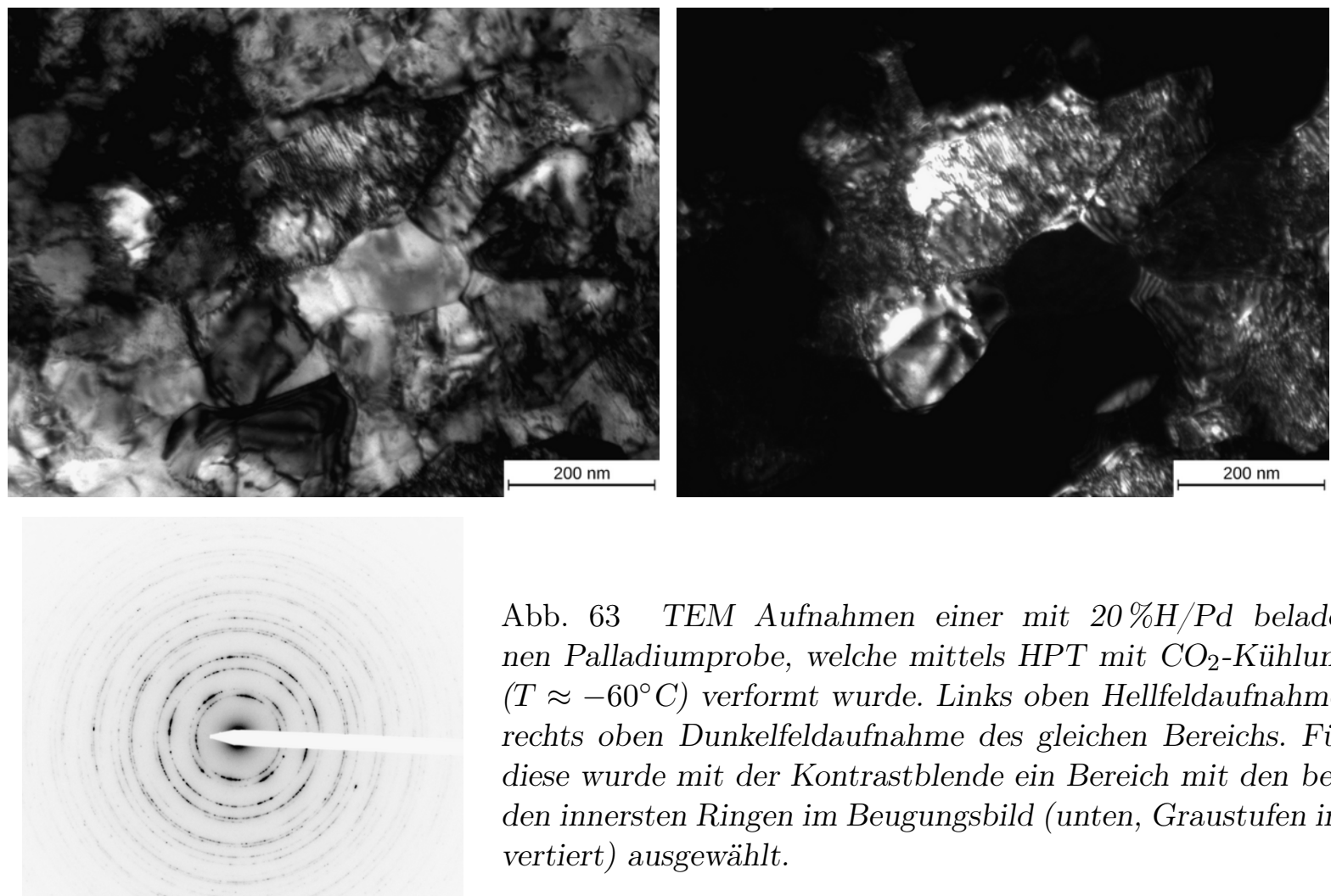

Abb. 63 TEM Aufnahmen einer mit $20 \% \mathrm{H} / \mathrm{Pd}$ beladenen Palladiumprobe, welche mittels $\mathrm{HPT}$ mit $\mathrm{CO}_{2}$-Kühlung $\left(T \approx-60^{\circ} C\right)$ verformt wurde. Links oben Hellfeldaufnahme, rechts oben Dunkelfeldaufnahme des gleichen Bereichs. Für diese wurde mit der Kontrastblende ein Bereich mit den beiden innersten Ringen im Beugungsbild (unten, Graustufen invertiert) ausgewählt. 

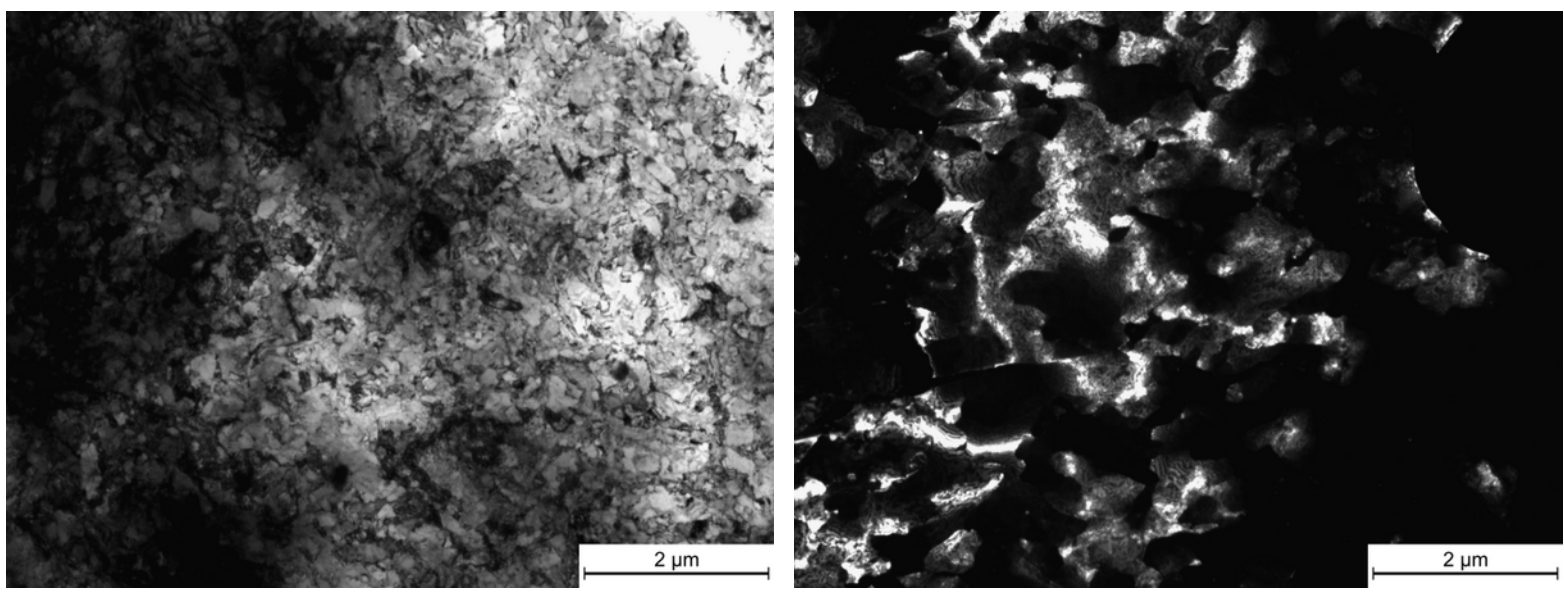

Abb. 64 Übersichtsaufnahmen einer mit 45\%H/Pd beladenen Palladiumprobe, welche mittels HPT bei Raumtemperatur verformt wurde. Links Hellfeldaufnahme, rechts

Dunkelfeldaufnahme.
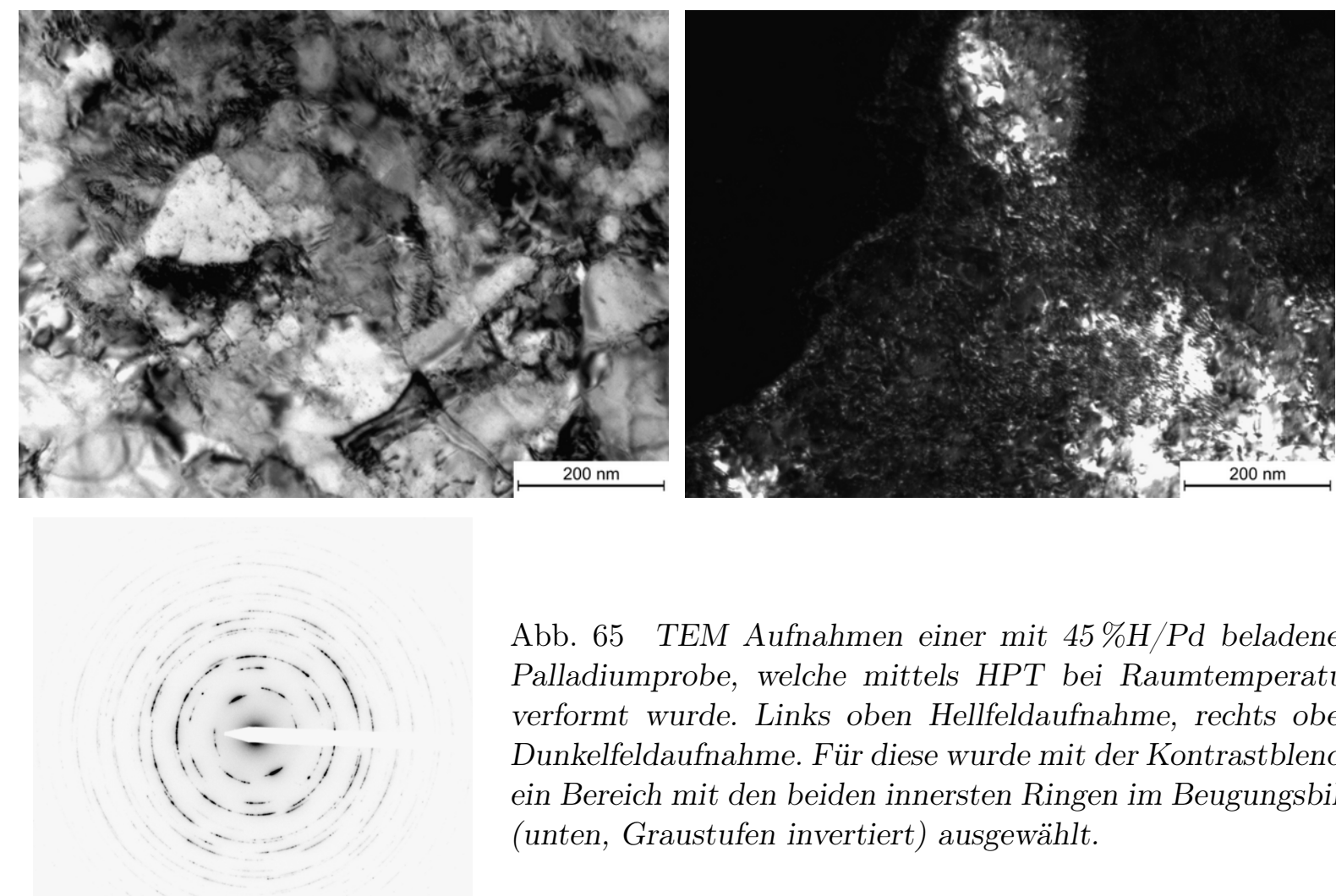

Abb. 65 TEM Aufnahmen einer mit $45 \% \mathrm{H} / \mathrm{Pd}$ beladenen Palladiumprobe, welche mittels HPT bei Raumtemperatur verformt wurde. Links oben Hellfeldaufnahme, rechts oben Dunkelfeldaufnahme. Für diese wurde mit der Kontrastblende ein Bereich mit den beiden innersten Ringen im Beugungsbild (unten, Graustufen invertiert) ausgewählt. 

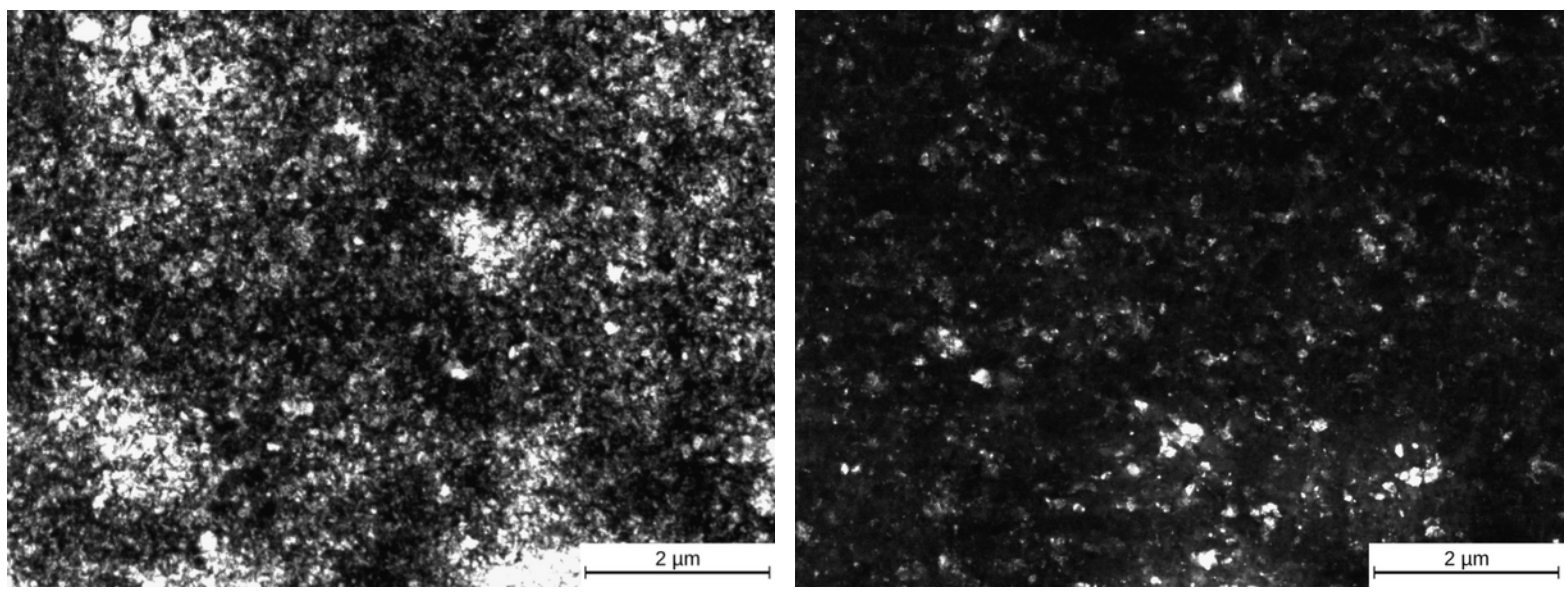

Abb. 66 Übersichtsaufnahmen einer mit $45 \% \mathrm{H} / \mathrm{Pd}$ beladenen Palladiumprobe, welche mittels HPT mit $\mathrm{CO}_{2}$-Kühlung $\left(T \approx-60^{\circ} \mathrm{C}\right)$ verformt wurde. Links Hellfeldaufnahme, rechts

Dunkelfeldaufnahme.
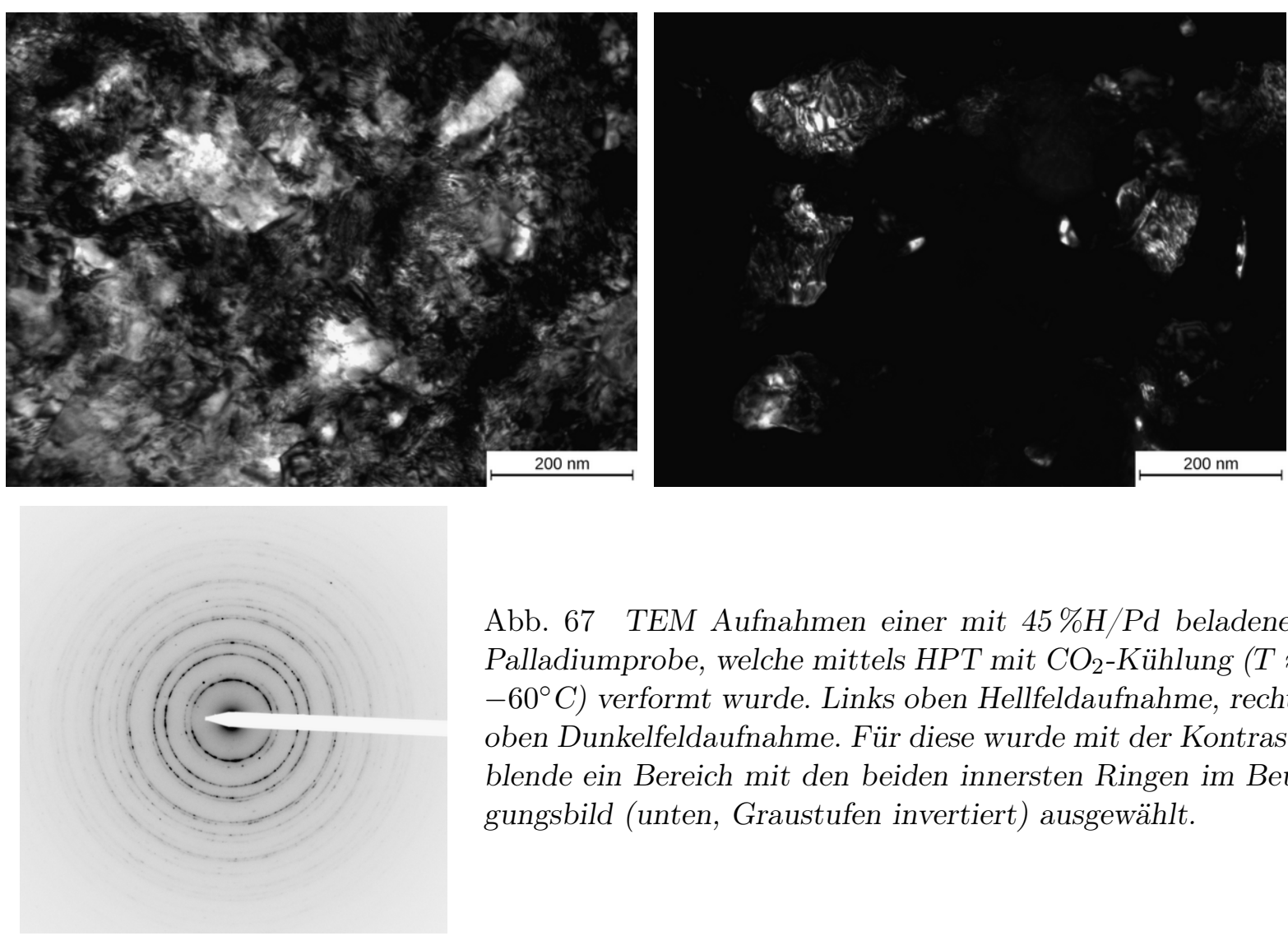

Abb. 67 TEM Aufnahmen einer mit $45 \% \mathrm{H} / \mathrm{Pd}$ beladenen Palladiumprobe, welche mittels $\mathrm{HPT}$ mit $\mathrm{CO}_{2}$-Kühlung $(T \approx$ $-60^{\circ} \mathrm{C}$ ) verformt wurde. Links oben Hellfeldaufnahme, rechts oben Dunkelfeldaufnahme. Für diese wurde mit der Kontrastblende ein Bereich mit den beiden innersten Ringen im Beugungsbild (unten, Graustufen invertiert) ausgewählt. 


\subsection{Analyse der Abgleitung von Mikrosäulen unter Verwendung des Schmidfaktors}

Für die Interpretation der Bilder von verformten Säulen ist der Schmid-Faktor eine nützliche Hilfe. Unter uniaxialer Spannung verformt sich ein einkristallines Material, indem sich Versetzungen auf geeigneten Gleitebenen bewegen. Diese Gleitebenen sind die am dichtesten besetzten Flächen im Kristallgitter. Läuft eine Versetzung auf einer Gleitebene, wird der obere gegenüber dem unteren Teil um einen Burgersvektor verschoben. Dieser Prozess wird Abgleiten genannt.

Im kubisch flächenzentrierten Gitter (kfz) gibt es zwölf kristallografisch gleichberechtigte Gleitsysteme. Die Gleitebenen sind vom Typ $\{111\}$, d. h. es gibt vier verschiedene Ebenenscharen und die Gleitrichtungen sind vom Typ $\langle 110\rangle$, d. h. in jeder Gleitebene gibt es drei $\langle 110\rangle$-Richtungen, die senkrecht zur Ebenennormale sind.

Für das raumzentrierte Gitter (krz) gibt es zwölf gleichberechtigte Gleitsysteme. Die Gleitebenen sind vom Typ $\{110\}$ und die Gleitrichtungen sind vom Typ $\langle 111\rangle$, ferner sind die Gleitsysteme $\{112\},\langle 111\rangle$ und $\{123\},\langle 111\rangle$ möglich [45].

Welches Gleitsystem bei einer konkreten Belastungsrichtung aktiviert wird ${ }^{34}$, kann anhand des Schmid-Faktors berechnet werden, vgl. hierzu Abbildung 68.

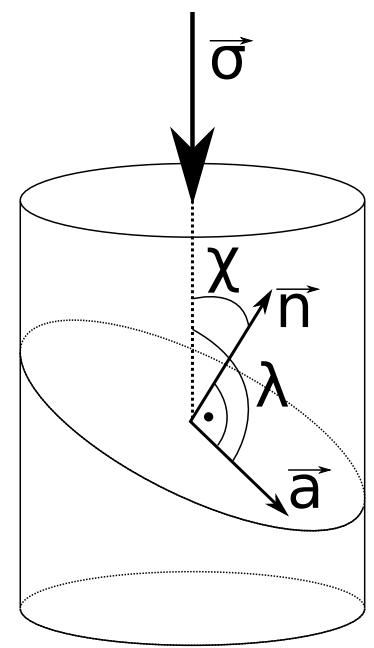

Abb. 68 Schematische Darstellung der Kräfteverhältnisse bei uniaxialer Belastung $\sigma$. Die einzelnen Kristallebenen erfahren entsprechend

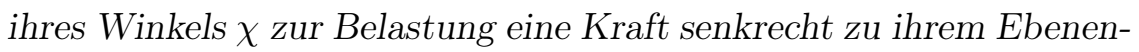
vektor $\vec{n}$, welcher durch Gleiten in geeignete Richtungen $\vec{a}$ abgebaut werden kann. Der Winkel $\lambda$ gibt dabei den Winkel zwischen der Abgleitebene und der Belastungsrichtung an.

Der Schmid-Faktor $m_{s}$ gibt an, wie wahrscheinlich es ist, dass das zugehörige Gleitsystem beim Verformen abgleitet, und kann Werte zwischen 0 und 0,5 annehmen. Für die Scherspannung $\tau$ bei uniaxialer Belastung gilt:

$$
\begin{aligned}
\tau & =\frac{\text { Kraft }}{\text { Fläche }}=\frac{F \cos (\lambda)}{A / \cos (\chi)} \\
& =\frac{F}{A} \cos \lambda \cos \chi \\
& =\frac{F}{A} m_{s}
\end{aligned}
$$

$\lambda$ beschreibt den Winkel zwischen einer mögliche Gleitrichtung $\vec{a}$ und der Belastungsrichtung $\vec{\sigma}$. $\chi$ beschreibt den Winkel zwischen der Gleitebenennormalen $\vec{n}$ und $\vec{\sigma}$ (vgl. Abbildung 68).

Der Schmidfaktor wird wie folgt definiert:

$$
\begin{aligned}
\vec{\sigma} \vec{n} & =|\vec{\sigma}||\vec{n}| \cos (\chi) \\
\vec{\sigma} \vec{a} & =|\vec{\sigma}||\vec{a}| \cos (\lambda) \\
\Rightarrow m_{s} & =\frac{(\vec{\sigma} \cdot \vec{a})(\vec{\sigma} \cdot \vec{a})}{|\vec{\sigma}|^{2}|\vec{n}||\vec{a}|}
\end{aligned}
$$

\footnotetext{
${ }^{34}$ Es wird von einem idealen Festkörper ausgegangen. Sind Defekte vorhanden, verändern sich die lokalen Spannungsfelder und das von außen beobachtete Verhalten muss nicht mit dem theoretischen Verhalten, berechnet aus der außen anliegenden Spannung, übereinstimmen.
} 


\section{4 Übersicht über betreute Bachelorarbeiten}

\subsubsection{Annegret Lehmberg - Untersuchung der Volumenänderung von Eisen nach Wasserstoffbeladung}

\section{Zusammenfassung der Arbeit:}

In der im Rahmen dieser Arbeit betreuten Bachelorarbeit von Annegret Lehmberg [178] wurde die Entstehung von Rissen in Eisen durch Wasserstoffbeladung untersucht. Dazu wurde hochreines Eisen bei sehr hohen elektrischen Stromdichten (bis zu $100 \mathrm{~mA} / \mathrm{cm}^{2}$ ) elektrolytisch beladen. Dies führt zur Bildung von Wölbungen, sog. Blistern, an der Oberfläche der Proben. Diese können auf die Entstehung von darunterliegenden Rissen zurückgeführt werden.

Die Arbeit gliedert sich in zwei Bereiche: Zum einen wurde ein hochpräziser Aufbau zur Dichtebestimmung nach dem Archimedischen Prinzip konstruiert, mit dem es möglich ist die Dichte von 1-2 g schweren Proben mit einem Fehler von unter einem Prozent zu bestimmen. Außerdem ist es mit diesem Aufbau möglich, Gewichtsveränderungen über lange Zeiträume zu beobachten. Dies erlaubt es, den zeitlichen Verlauf des Wasserstoffaustritts aus beladenen Proben zu bestimmen.

Zum anderen wurden die entstandenen Risse und Blister anhand von rasterelektronenmikroskopischen Aufnahmen charakterisiert. Auch wurde untersucht, unter welchen Voraussetzungen Blister entstehen.

Das Hauptergebnis ist die Feststellung, dass alleinig die Beladung mit Wasserstoff ausreicht, um bei genügend hohen Beladeströmen Risse in Eisen zu bilden.

Durch die Messung des Gewichts und der Dichte ist es zudem möglich, den Wasserstoffdruck in den Rissen kurz nach der Beladung zu bestimmen. Dieser liegt im Bereich von $100 \mathrm{MPa}$, was im Bereich der Fließgrenze von Eisen liegt (76-97 MPa [180, 181]). Diese Erkenntnis erlaubt es ein Modell der Rissbildung aufzustellen: Gelöster Wasserstoff diffundiert zu bereits im Material vorhandenen Hohlräumen und rekombiniert dort zu Wasserstoffgas. Dies führt zu einem Druckanstieg. Ist der Druck höher als die Fließgrenze, reißt der Hohlraum auf. Danach diffundiert weiterer Wasserstoff in den Riss bis wieder ein genügend großer Druck entstanden ist. Es kommt zu einer weiteren Rissausbreitung. Ein weiterentwickeltes Modell zur Entstehung von Rissen in Eisen durch Wasserstoff wird in [182] beschrieben. Die hier vorgestellten Ergebnisse und der genaue experimentelle Ablauf sind in [18] veröffentlicht worden.

\section{Beschreibung und Ergebnisse der Versuche:}

Die verwendeten Proben hatten eine Reinheit von 99,9\% und wurden vor der Untersuchung im Vakuum $\left(<10^{-6} \mathrm{mbar}\right)$ bei $880^{\circ} \mathrm{C}$ für 5 Stunden ausgelagert und anschließend im Ofen abgekühlt. Die so erhaltenen Proben wurden danach elektrolytisch bei verschiedenen Stromdichten im Bereich von $1-100 \mathrm{~mA} / \mathrm{cm}^{2}$ in 0,1 molare Schwefelsäure $\left(\mathrm{H}_{2} \mathrm{SO}_{4}\right)$ mit einem Wasserstoffpromoter $(0,1$ Gew.\% Ammoniumthiocyanat $\left.\left(\mathrm{NH}_{4} \mathrm{SCN}\right)\right)$ beladen.

Direkt nach dem Beladen wurden die Proben auf eine Waage gelegt und der zeitliche Verlauf des Wasserstoffaustritts über die Änderung des Gewichts bestimmt, siehe Abbildung 69. Nachdem der Wasserstoff aus der Probe hinaus diffundiert ist, wurde die Dichte nach der Methode von Archimedes bestimmt, indem der Auftrieb in einer schweren Flüssigkeit (Natriumpoly-wolframatLösung $\left.\left(\mathrm{Na}_{6}\left(\mathrm{H}_{2} \mathrm{~W}_{1} 2 \mathrm{O}_{4} 0\right) \cdot \mathrm{H}_{2} \mathrm{O}\right)\right)$ bestimmt wurde. Mit der Dichte vor und nach der Beladung und dem Gewicht der Probe (ohne Wasserstoff) kann auf das Volumen der Risse geschlossen werden. Da auch das Gewicht der Probe kurz nach der Beladung bekannt ist, kann mit Hilfe des idealen Gasgesetzes der Druck des Wasserstoffs in den Rissen abgeschätzt werden. Dieser liegt im Bereich von $100 \mathrm{MPa}$ und somit im Bereich der Fließgrenze von reinem Eisen. 


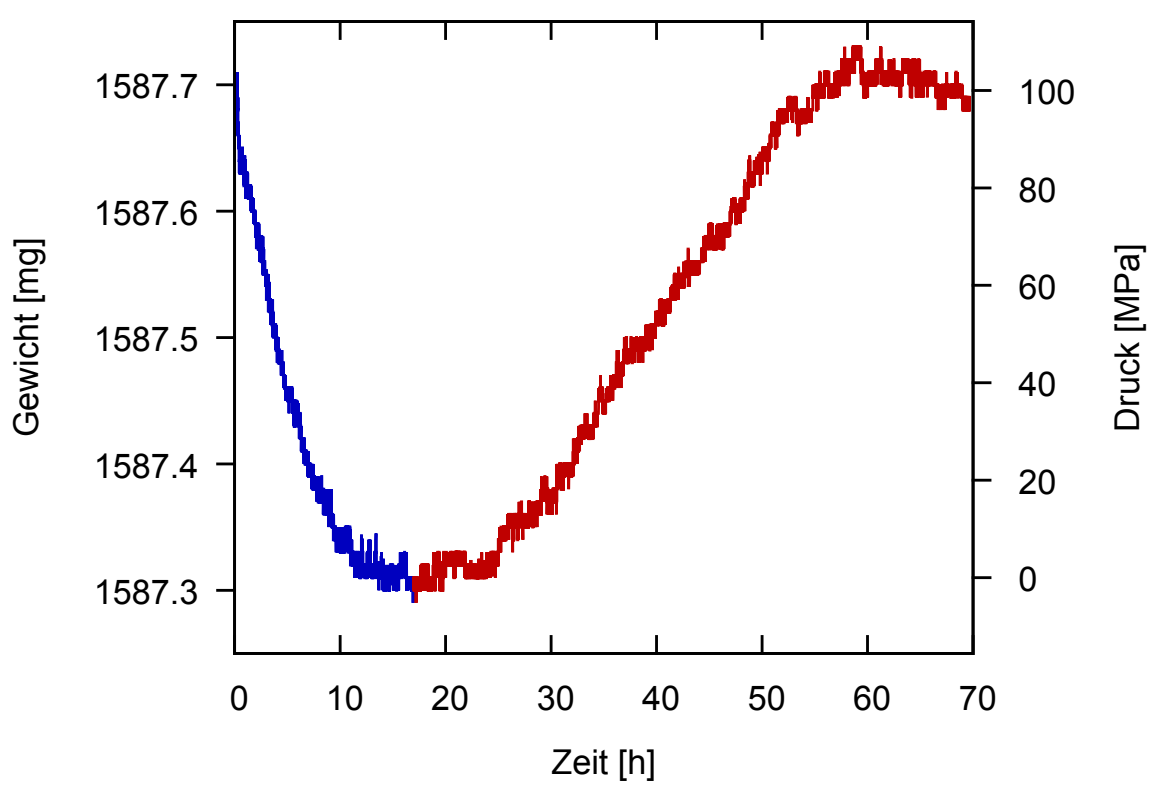

Abb. 69 Zeitlicher Verlauf des Gewichtes einer mit Wasserstoff beladenen Probe. Zunächst fällt das Gewicht (blau), da Wasserstoff aus der Probe desorbiert. Danach steigt das Gewicht (rot). Dies wird auf Oxidation der Probe zurückgeführt. Der Gasdruck des Wasserstoffs in den Rissen bei fallendem Gewicht (blau) kann mit dem idealen Gasgesetz abgeschätzt werden.

Des Weiteren wurden die entstandenen Blister an der Oberfläche (Abbildung 70) untersucht. Es zeigte sich, dass die Blister Durchmesser von bis zu 0,3 mm haben und sich nicht an Korngrenzen orientieren. Bei genauerer Untersuchung der Blister durch Marie Tiegel [18] zeigte sich zudem, dass die Ränder der Blister viele Stufen aufweisen und die eingeschlossene Oberfläche unverändert bleibt.
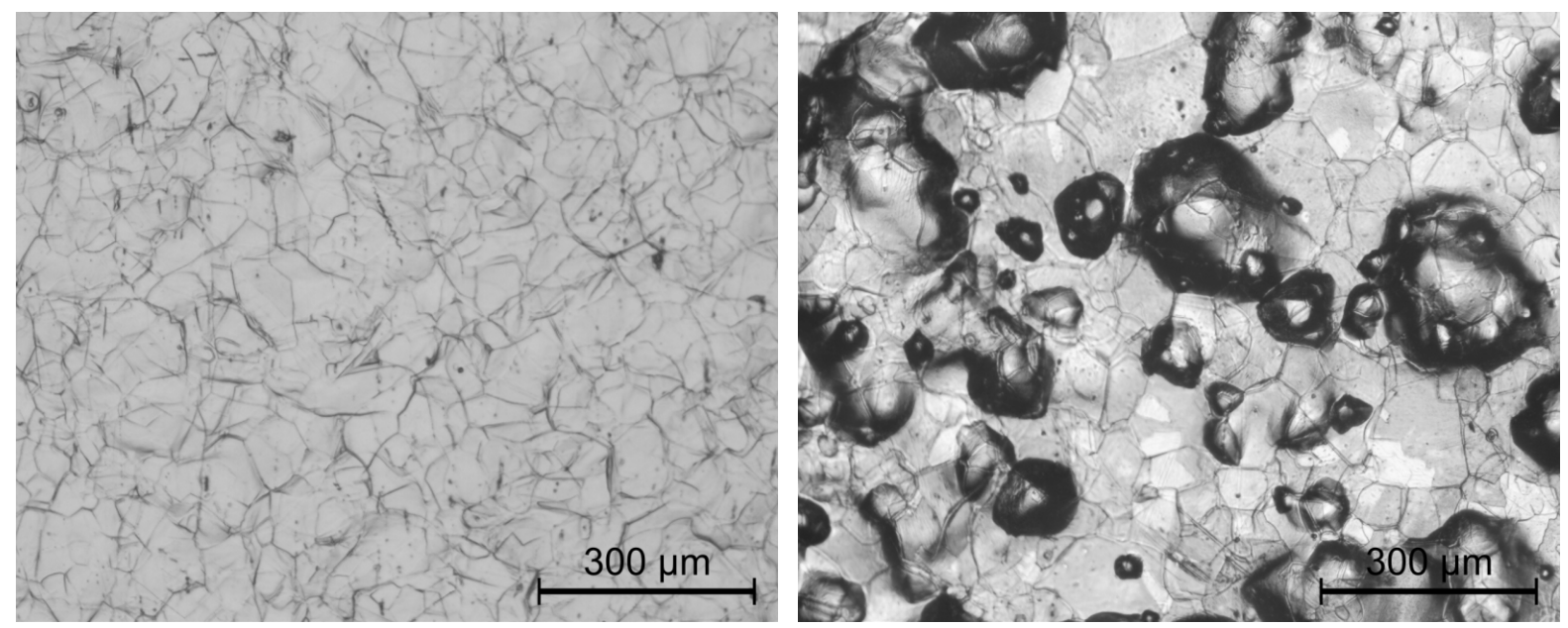

Abb. 70 Lichtmikroskopische Aufnahmen von einer Oberfläche einer Eisenprobe. Links: Nicht beladenes Eisen; Rechts: Eisen beladen mit Wasserstoff bei $100 \mathrm{~mA} / \mathrm{cm}^{2}$ für eine Stunde.

Um die Struktur der sich im Material befindlichen Risse zu analysieren, wurden Querschliffe angefertigt (siehe Abbildung 71). Zu erkennen ist, dass im gesamten Bereich der Probe (Probendicke: $600 \mu \mathrm{m})$ Risse entstanden sind. Jedoch sind diese Risse vermehrt in der Nähe der Oberfläche zu finden. Es fällt auf, dass über diesen Rissen nahe zur Oberfläche Blister zu finden sind. Bei 
Detailaufnahmen von einzelnen Rissen (Abbildung 72) ist zu erkennen, dass diese sich nicht an Korngrenzen orientieren und oft verzweigt auftreten.

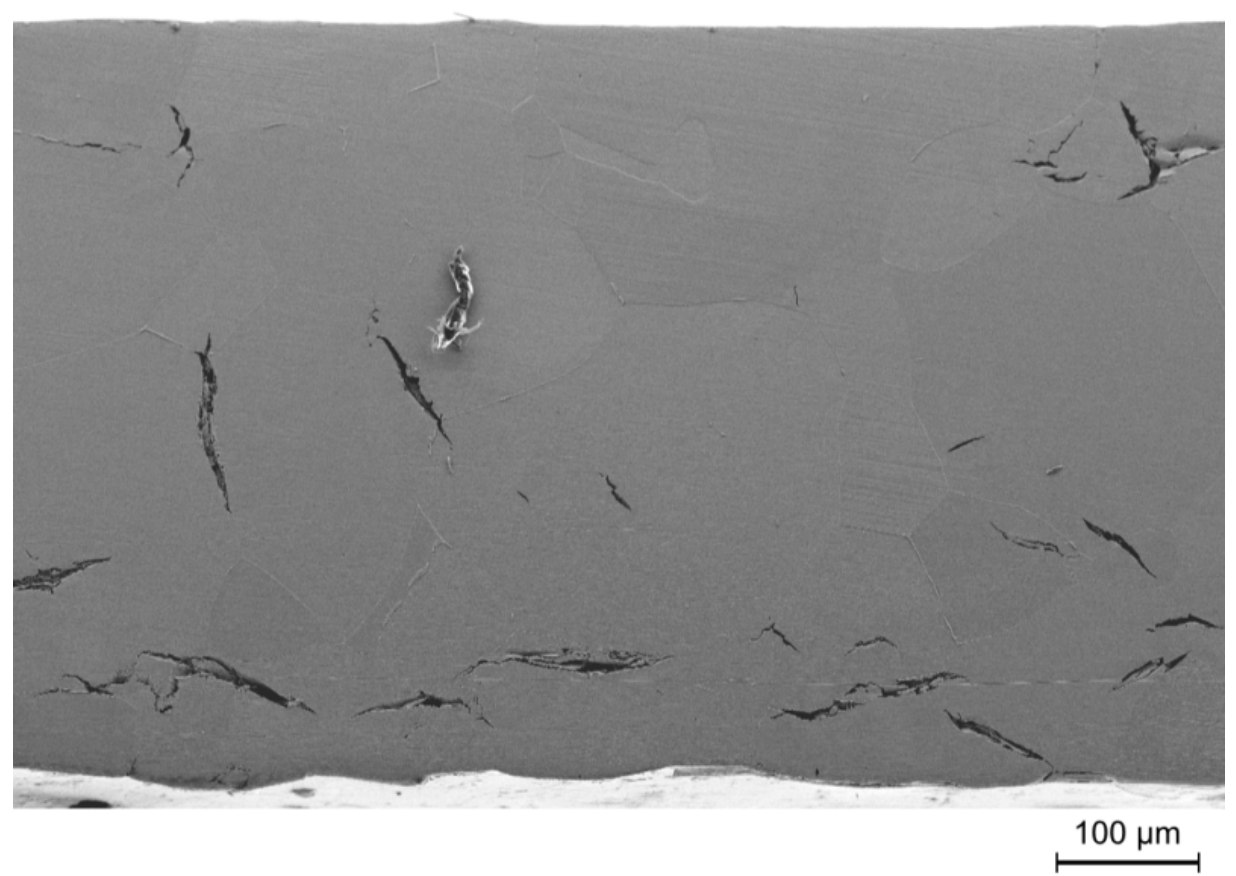

Abb. 71 Querschnittsfläche einer mit $100 \mathrm{~mA} / \mathrm{cm}^{2}$ für 1 Stunde mit Wasserstoff beladenen Eisenprobe, (Angefertigt von Lennart Quintscher, IMP, Uni Göttingen). Zu erkennen sind mehrere Risse, welche sich unabhängig von den Korngrenzen ausgebreitet haben. An den Oberflächen (helle Bereiche oben und unten) sind mehrere Wölbungen zu erkennen, unter denen Risse liegen. Diese Kombination von Riss und Wölbung ist ein Blister.

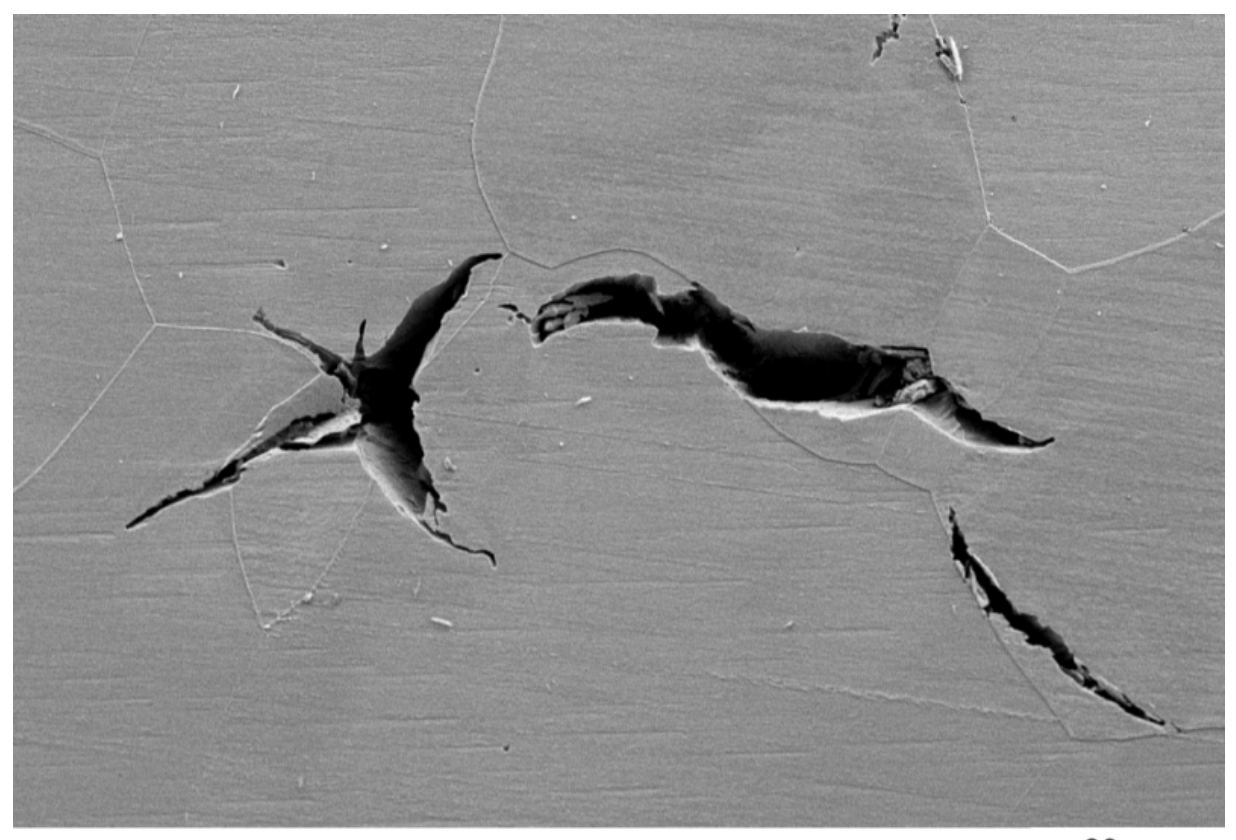

$30 \mu \mathrm{m}$

Abb. 72 Detailaufnahme mehrerer Risse in einer mit $100 \mathrm{~mA} / \mathrm{cm}^{2}$ für 1 Stunde mit Wasserstoff beladenen Eisenprobe (Angefertigt von Lennart Quintscher, IMP, Uni Göttingen). Die Risse scheinen nicht von Korngrenzen beeinflusst zu werden. Einige Risse zeigen eine sternförmige

Struktur. 


\subsubsection{Malte Lenz - Zusammenhang zwischen der Mikrostruktur und den mechanischen Eigenschaften sprengverschweißter Stähle}

\section{Zusammenfassung der Arbeit:}

In der Bachelorarbeit von Malte Lenz [186] wurde die Mikrostruktur einer Schweißnaht, welche durch Explosivschweißen erzeugt wurde, charakterisiert.

Die Proben wurden im Rahmen der Doktorarbeit von Dr. Hammerschmidt hergestellt [187] und bestehen aus einem perlitarmen Stahl (PAS), auf welchen mittels Explosivschweißen ein Blech aus C45-Stahl aufgebracht wurde.

In Abbildung 73 ist das Verfahren schematisch dargestellt. Hierbei wird eine kontrollierte Explosion genutzt, um eine sog. Flugplatte unter einem Winkel auf eine Grundplatte zu beschleunigen. Die Flugplatte erreicht Geschwindigkeiten $\left(v_{a}\right)$ von mehreren hundert Metern pro Sekunde. Die Geschwindigkeit der Detonationsfront $\left(v_{b}\right)$ wird durch die Auswahl des Sprengstoffs bestimmt. Eine tiefer gehende Beschreibung des Verfahrens kann in den Arbeiten von K. Keller [188-190] nachvollzogen werden.

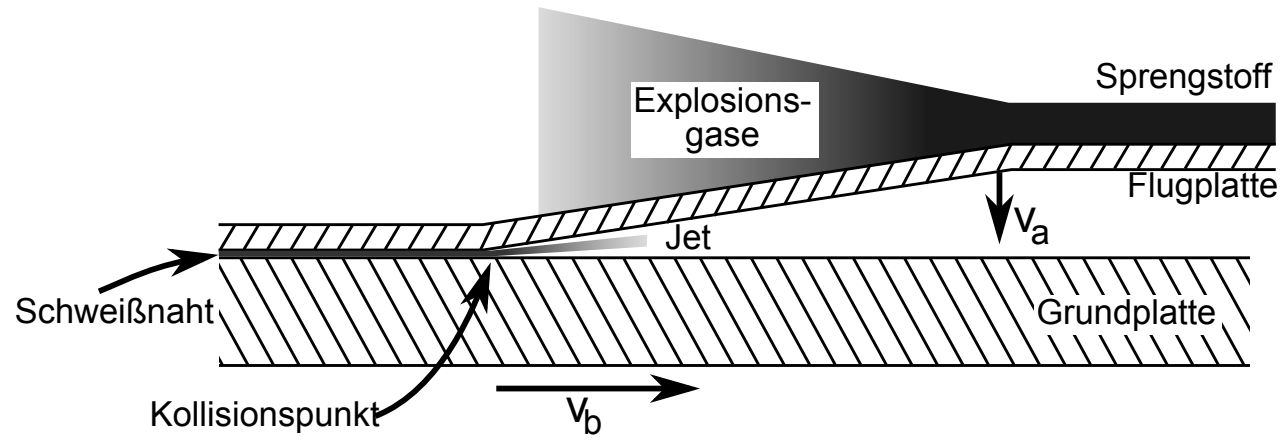

Abb. 73 Schematische Darstellung des Explosivschweißverfahrens [191].

Ziel der Arbeit war es die mikrostrukturellen Ursachen für die besonders hohe Festigkeit der durch dieses Verfahren erzeugten Schweißnaht zu ermitteln. Hierzu wurde zunächst ein Querschliff angefertigt (Abbildung 74). Dieser zeigt, dass die Schweißnaht einer Wellenlinie folgt. Zwischen beiden Materialien bildet sich ein neues Material aus, welches keinem der Ausgangsmaterialien zugeordnet werden kann. TEM-Untersuchungen (Abbildung 77) ergeben, dass hier eine sehr hohe Versetzungsdichte vorliegt. Des Weiteren zeigen die TEM-Aufnahmen, dass nur im Bereich der Schweißnaht Veränderungen in der Mikrostruktur erfolgt sind. Daraus und aus der Form der Wellen kann erahnt werden, dass eine enorme plastische Verformung stattgefunden haben muss.

Durch Härtemessungen (Einschub in Abbildung 74) konnte gezeigt werden, dass diese Grenzschicht mehr als doppelt so hart ist, wie die beiden Stähle.

Um die Festigkeit der Probe zu untersuchen wurden Nanoindentationsversuche und Zugversuche durchgeführt. Die Nanoindentation ergibt, dass die Bereiche der Schweißnaht doppelt so hart sind, wie die Ausgangsmaterialien. Für die Ausgangsmaterialien ergab sich eine Härte von 3, $5 \pm 0,6 \mathrm{GPa}$ für C45-Stahl und 3,4 0, 4 für den PAS-Stahl. Bei der Schweißnaht wurde ein maximaler Wert von $7,9 \mathrm{GPa}$ gemessen. 


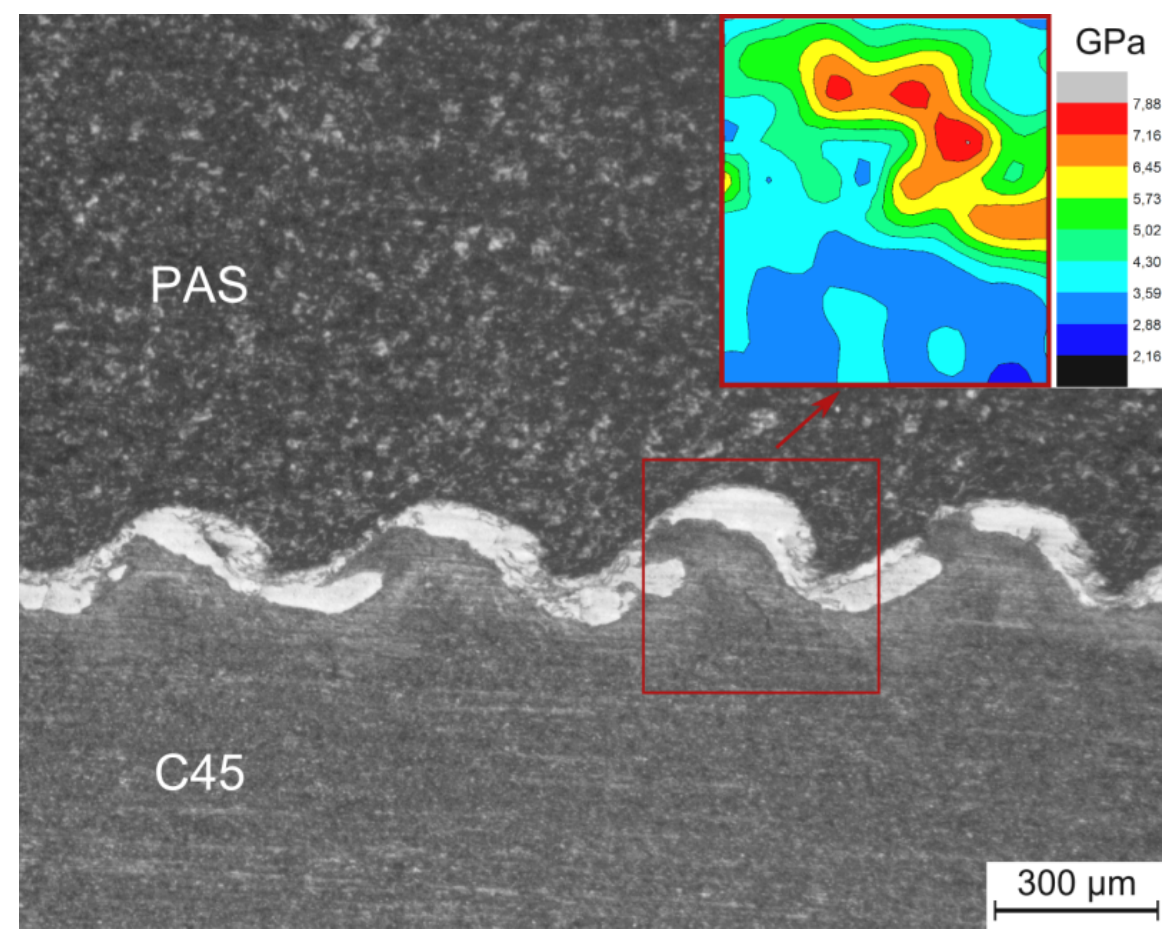

Abb. 74 Lichtmikroskopische Aufnahme eines Querschliffs einer durch Explosivschweißen von C45-Stahl auf perlitarmem Stahl entstandene Schweißnaht. Der Einschub zeigt die lokale Härte der Probe, welche durch Nanoindentation ermittelt wurde.

Für die Zugversuche wurden mittels Funkenerosion H-förmige Proben aus der Schweißnaht präpariert. Dabei wurden Proben senkrecht zur Schweißnaht und Proben waagerecht zur Schweißnaht präpariert. Die Ergebnisse sind in den Abbildungen 75 und $76 \mathrm{zu}$ sehen. Es zeigt sich, dass die Proben mit der waagerechten Schweißnaht mit dem E-Modul des C45-Stahls verformen und außermittig in den Bereich des C45-Stahls reißen. Die Zugversuche für die Proben, mit der senkrechten Schweißnaht, zeigen eine erhöhte Zugfestigkeit.
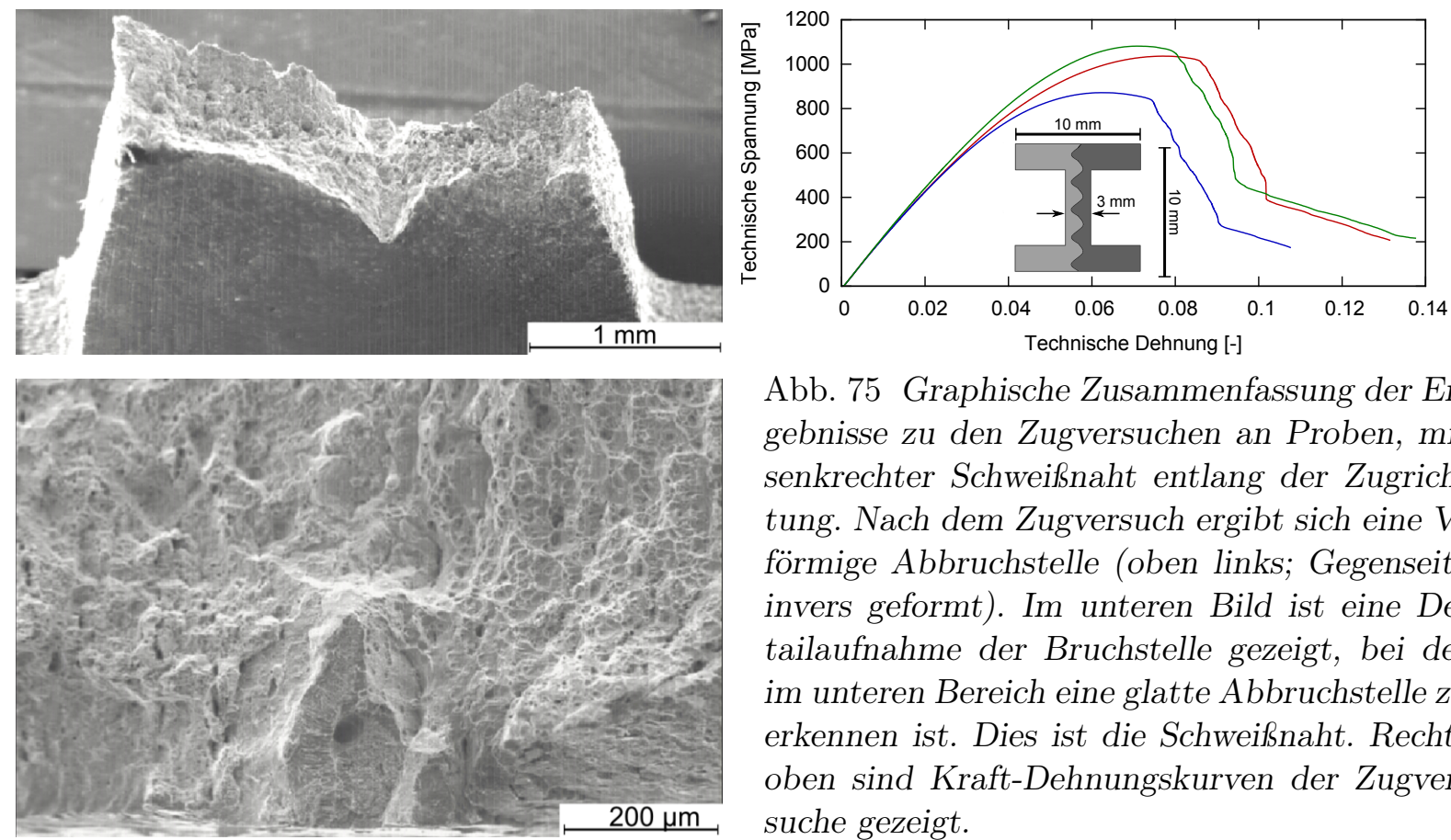

Abb. 75 Graphische Zusammenfassung der Ergebnisse zu den Zugversuchen an Proben, mit senkrechter Schweißnaht entlang der Zugrichtung. Nach dem Zugversuch ergibt sich eine $V$ förmige Abbruchstelle (oben links; Gegenseite invers geformt). Im unteren Bild ist eine Detailaufnahme der Bruchstelle gezeigt, bei der im unteren Bereich eine glatte Abbruchstelle zu erkennen ist. Dies ist die Schweißnaht. Rechts oben sind Kraft-Dehnungskurven der Zugversuche gezeigt. 

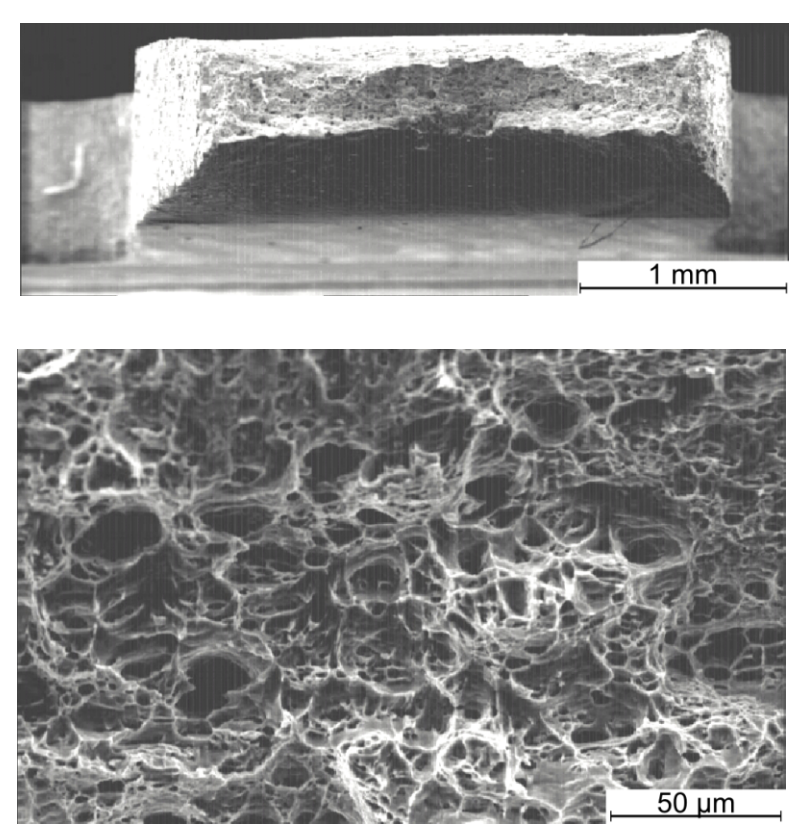

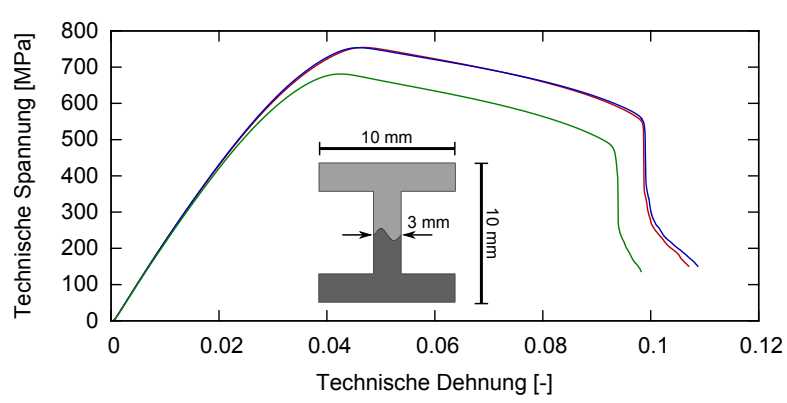

Abb. 76 Graphische Zusammenfassung der Ergebnisse zu den Zugversuchen an Proben mit waagerechter Schweißnaht. Nach dem Zugversuch ergibt sich eine duktile Abbruchstelle (oben links). Im unteren Bild ist eine Detailaufnahme der Bruchstelle gezeigt. Der Riss verläuft bei diesen Proben im C45-Stahl. Rechts oben sind Kraft-Dehnungskurven der Zugversuche gezeigt.

REM-Aufnahmen von den Bruchflächen (Abbildung 75) zeigen im Bereich der Schweißnaht glatte Bruchflächen, was auf sprödes Verhalten hinweist. Die Bereiche um die Schweißnaht (Abbildung 75) und die Bruchflächen der Proben mit waagerechter Schweißnaht (Abbildung 76) zeigen eine wabenartige Struktur, was ein Zeichen für duktiles Reißen ist.

Dies bedeutet, dass die Schweißnaht sehr hohen Belastungen standhält. Zum einen ist die Naht fester als die einzelnen Materialien (vgl. Abbildung 76), zum anderen verhält sich die Naht spröde, wodurch nur geringe Dehnungen möglich sind, bis die Naht reift und das übrige Material sich duktil verformt.

Die Ergebnisse dieser Arbeit wurden in [191] veröffentlicht.
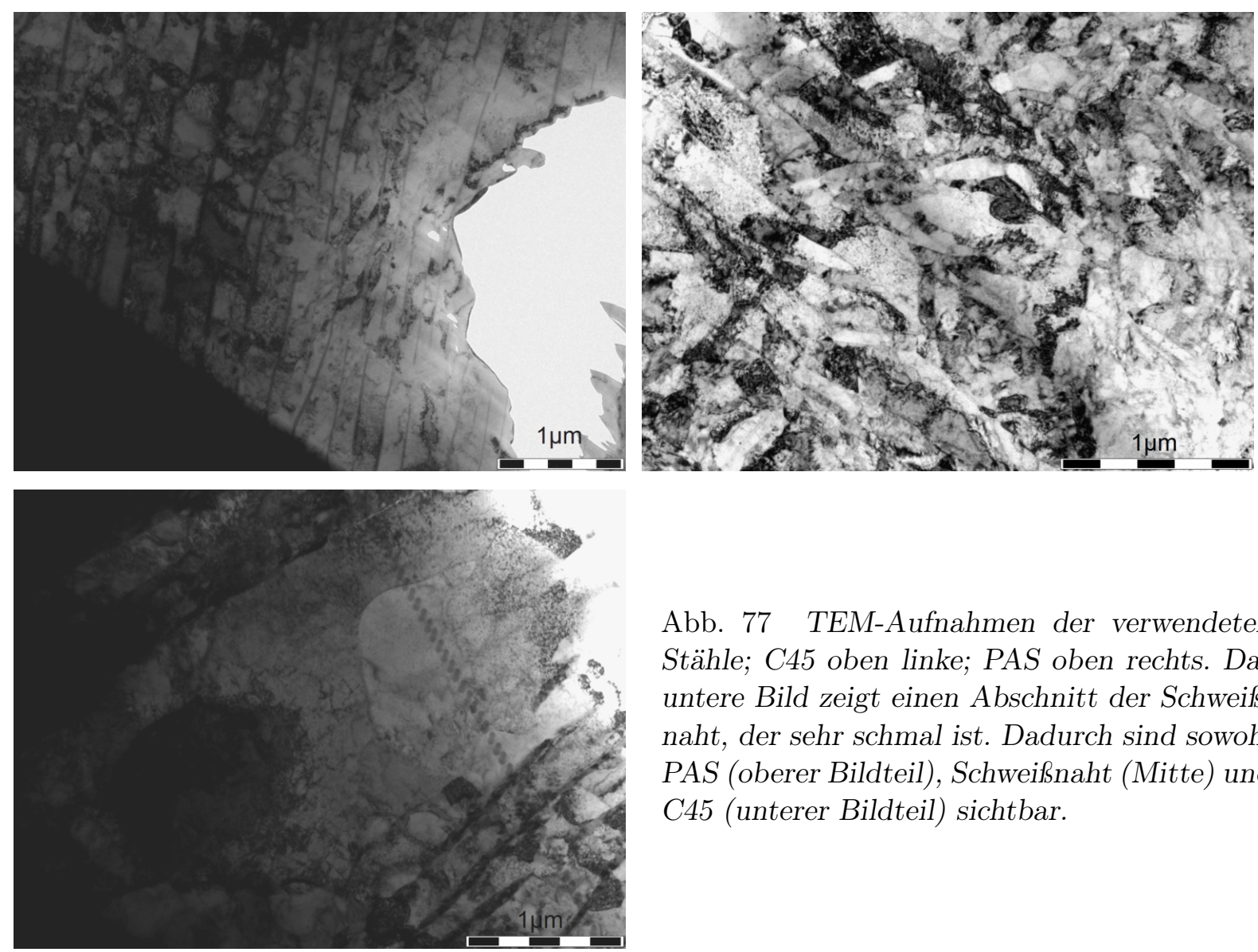

Abb. 77 TEM-Aufnahmen der verwendeten Stähle; C45 oben linke; PAS oben rechts. Das untere Bild zeigt einen Abschnitt der Schweißnaht, der sehr schmal ist. Dadurch sind sowohl PAS (oberer Bildteil), Schweißnaht (Mitte) und C45 (unterer Bildteil) sichtbar. 


\subsubsection{Moritz Roscher - Einfluss der Wasserstoffbeladung auf die mechanischen Eigenschaften von Metallen}

\section{Hintergrund der Arbeit}

Der Einfluss von Wasserstoff auf Zugexperimente ist schon lange Gegenstand von wissenschaftlichen Untersuchungen [1]. Hierbei zeigt sich, dass viele Materialien, welche sich normalerweise duktil verformen, durch Wasserstoff sprödes Verhalten zeigen, z. B. Stahl [100, 192-197], Eisen [198], Nickel [13, 14, 131, 199-201], Aluminium [42], Vanadium und Niob [202].

Um dieses Verhalten nachvollziehen zu können und um die Auswirkung von mechanischer Spannung und in Metall gelösten Wasserstoff auf die Defektbildung zu untersuchen, wurde in der im Rahmen dieser Arbeit betreuten Bachelorarbeit von Moritz Roscher [179] eine konventionelle Zugmaschine (Zwick 1474 der Firma Zwick/Roell) so modifiziert, dass Zugversuche innerhalb eines Elektrolyten durchgeführt werden können. Dieser Aufbau wurde verwendet, um Zugproben aus Nickel, Vanadium und Tantal zu verformen. Dabei wurden die Proben ohne und mit einer hohen Wasserstoffkonzentration im jeweiligen Einphasengebiet der Metall-Wasserstoffsysteme untersucht.

Neben der Aufnahme von Kraft-Dehnungskurven wurden die Bruchflächen nach den Zugexperimenten untersucht. Des Weiteren wurde mit einer Mikroskopkamera die Oberfläche der Probe beim Ziehen beobachtet.
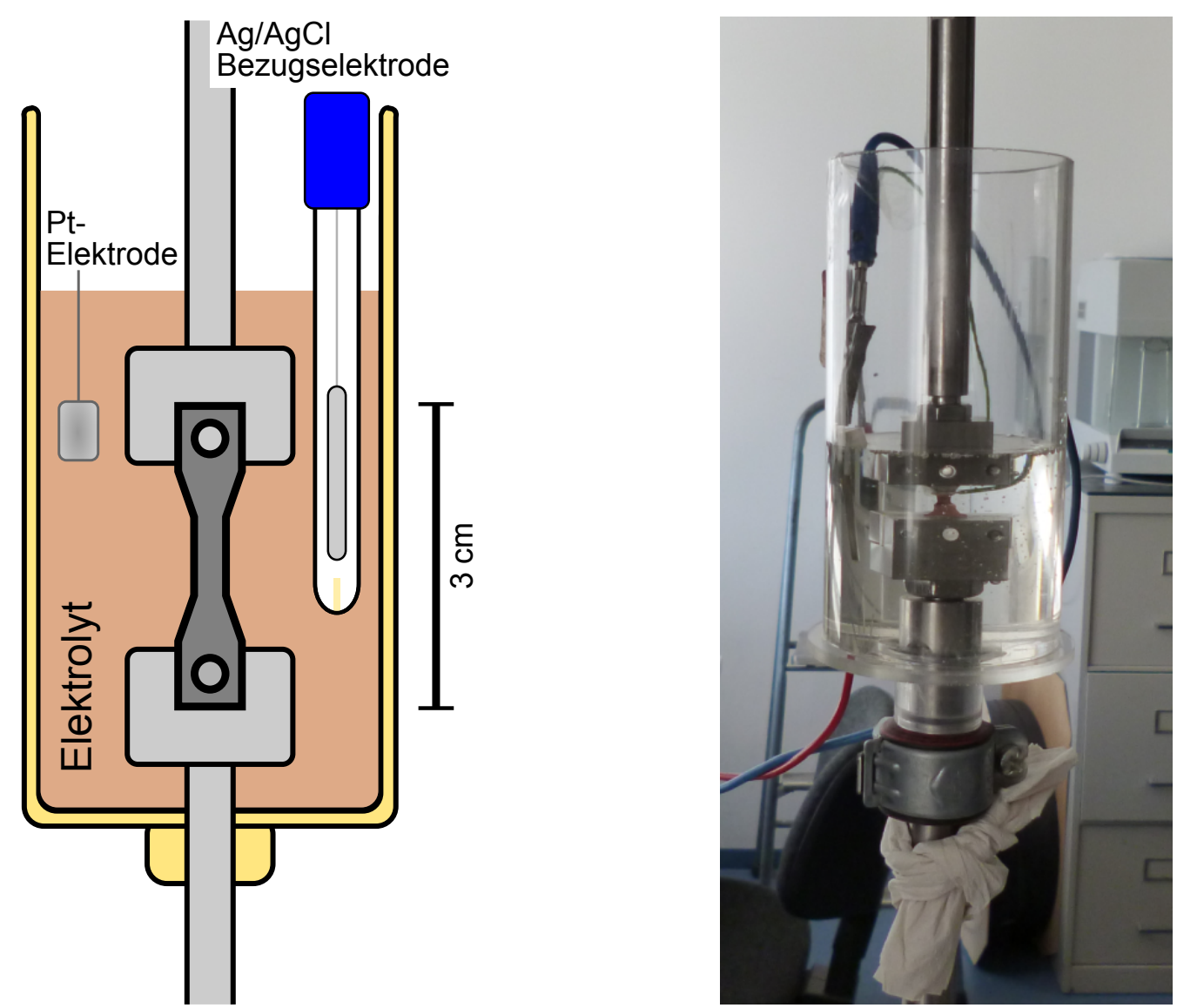

Abb. 78 Schematischer Aufbau (links) zur Durchführung von Zugversuchen im Elektrolyten und Foto des Aufbaus (rechts). Dieser Aufbau ermöglicht Proben während des Zugversuchs mit

Wasserstoff zu beladen, die elektromotorische Kraft zu bestimmen und gleichzeitig die Oberfläche mit einer Mikroskopkamera zu beobachten. 


\section{Aufbau des in-situ Zugexperiments}

In Abbildung 78 ist der in der Bachelorarbeit konstruierte Aufbau dargestellt. Dieser Aufbau wurde mit Hilfe von Florian Oelkers ${ }^{35}$ entwickelt und umgesetzt. Dieser Aufbau besteht aus einem Plexiglasgefäß mit einer halbkreisförmigen Grundfläche. Dieses Gefäß ist am unteren Probengreifer befestigt und kann zum schnellen Probenwechsel auf und ab bewegt werden. Damit nur die Probe beladen wird und für zukünftige Experimente, bei denen die EMK mit einer Bezugselektrode gemessen werden soll, muss die Probe elektrisch isoliert von der Zugmaschine eingebaut sein. Daher wurden Abstandshalter aus Teflon an den Backen der Probengreifern angebracht. Da eine Probe durch Teflon nicht gehalten werden würde, wurden in die Greifer Löcher gebohrt. Durch diese werden die Proben mit Stäben, aus wahlweise Plexiglas, Glas oder Keramik, fixiert.

Das Gefäß wurde so groß gewählt, dass neben einer Platinanode auch eine Silber/Silberchlorid Bezugselektrode in den Elektrolyten eingelassen werden kann.

Die planare Oberfläche des Gefäßes erlaubt die Beobachtung der Probe mit einer Mikroskopkamera. Hierbei wurde eine maximale Vergrößerung von 200-facher Vergrößerung erreicht.

\section{Versuchsdurchführung}

Für die Untersuchungen wurden die Metalle Nickel ${ }^{36}$, Vanadium ${ }^{37}$ und Tantal ${ }^{38}$ verwendet. Aus dem entsprechenden Blech wurden mittels Funkenerosion die Proben ausgeschnitten, siehe Abbildung 79. Danach wurden die Proben geschliffen und anschließend in Vakuum (Druck $10^{-7}$ mbar) bei $900^{\circ} \mathrm{C}$ für 20 Stunden ausgelagert.

Nickel kann ohne weitere Probenpräparation so verwendet werden. Da die natürliche Oxidschicht von Vanadium [103] und Tantal eine Beladung mit Wasserstoff verhindert, wird eine Palladiumschicht aufgetragen, durch die die Beladung ermöglicht wird. Dazu wurde in einer Vakuumsputteranlage mit Hilfe von Magnus Hamm ${ }^{39}$ zunächst die Oxidschicht von einer Seite der Proben entfernt und anschließend eine $20 \mathrm{~nm}$ dicke Palladiumschicht aufgetragen.

Für die Beladung mit Wasserstoff wurde ein Elektrolyt bestehend aus 1/3 Phosphorsäure und 2/3 Glyzerin verwendet. Dieser wurde vor der Verwendung mindestens 12 Stunden mit Argon durchflutet, damit gelöster Sauerstoff verdrängt wird. Gelöster Sauerstoff im Elektrolyten würde dazu führen, dass Wasserstoff an der Oberfläche zu Wasser reagieren würde und somit die Wasserstoffkonzentration im Metall unbekannt wäre.

Die Proben wurden, wie in Kapitel 3.1.1 beschrieben, mit Wasserstoff beladen. Dabei wurde Nickel mit einer Stromdichte von $0,1 \mu \mathrm{A} / \mathrm{cm}^{2}$ und Vanadium und Tantal mit $0,3 \mathrm{~mA} / \mathrm{cm}^{2}$ beladen, wobei bei letzteren jedoch nur durch die mit Palladium beschichtete Fläche beladen werden kann. Danach werden die Proben mit einer Geschwindigkeit von $1 \mu \mathrm{m} / \mathrm{s}$ gedehnt.
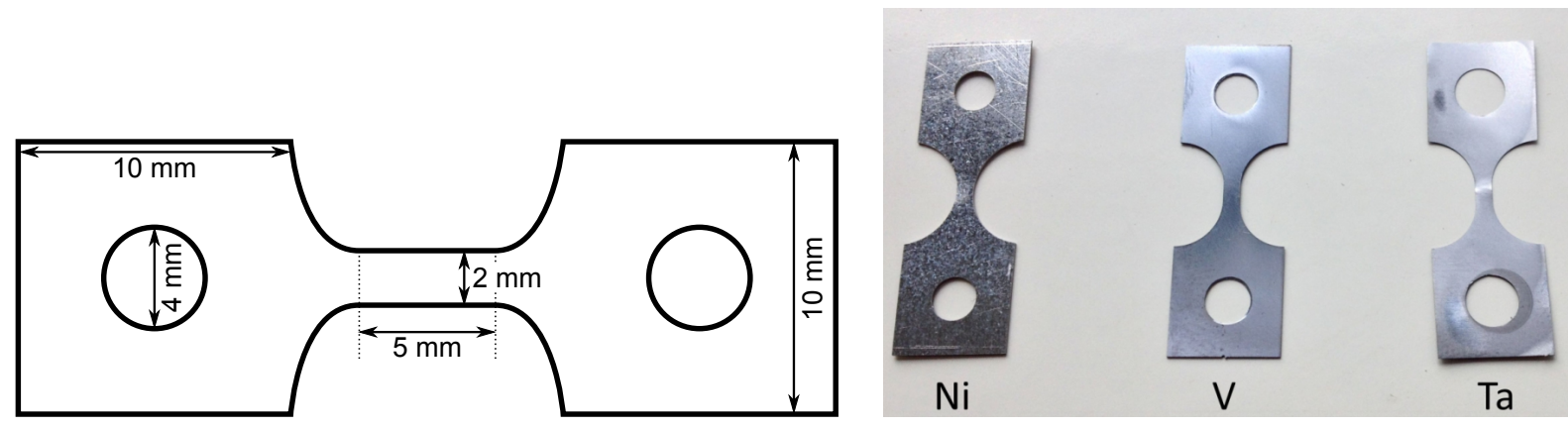

Abb. 79 Schematische Darstellung der verwendeten Proben (links), und Foto (rechts) der Proben aus den Metallen (v.l.n.r.) Nickel, Vanadium und Tantal.

\footnotetext{
${ }^{35}$ Institut für Materialphysik, Universität Göttingen

${ }^{36}$ Erworben bei Goodfellow mit einer Reinheit von $99,0 \%$ und Dicke von 0,3 mm

${ }^{37}$ Erworben bei Alfa Aesar mit einer Reinheit von $99,8 \%$ und Dicke von 0,25 mm

${ }^{38}$ Erworben bei ChemPur Feinchemikalien und Forschungsbedarf GmbH mit einer Reinheit von 99,9\% und Dicke von $0,1 \mathrm{~mm}$

${ }^{39}$ Institut für Materialphysik
} 


\section{Zusammenfassung der Ergebnisse}

\section{Zugversuche an Nickel:}

Nickel wurde ohne und mit einer Konzentration von $0,0012 \mathrm{H} / \mathrm{Ni}$ bzw. 0,0025 H/Ni untersucht. Nach [203] liegt die maximale Konzentration der Lösungsphase zwischen 0,0012 H/Ni und 0,0025 H/Ni. Die Proben ohne und mit einer Konzentration von $0,0012 \mathrm{H} / \mathrm{Ni}$ zeigen keinen großen Unterschied sowohl in den Spannungs-Dehnungskurven als auch beim Bruchverhalten. Die SpannungsDehnungskurven in Abbildung 80 zeigen viele Unregelmäßigkeiten im Anfangsbereich der Kurven. Es wird angenommen, dass diese Unregelmäßigkeiten aufgrund der verwendeten Stäbe zur Fixierung der Probe auftreten. Auch unter Verwendung anderer Materialien (Glas oder Keramik) für die Stäbe ändert sich dieses Verhalten nur wenig. Bei Verwendung dieser Materialien kommt jedoch das Problem hinzu, dass diese unkontrolliert brechen können.

Erst bei einer Konzentration von 0,0025 H/Ni wird eine deutliche Verringerung der Totaldehnung sichtbar. Dabei verhält sich diese Probe bis zum Bruch vergleichbar zu den anderen Versuchen.

In der Abbildung 81 ist die Mikrostruktur der Bruchkante und die Verformung der Oberfläche zu sehen. Die Ausschnitte aus einer Mikroskop-Videoaufnahme der Oberfläche beim Ziehen zeigen die Entstehung und die Entwicklung eines Bruches. Die Markierung (1) zeigt die Enden des mittleren Steges der Zugprobe. Erste Rissanfänge sind mit (2) markiert. Diese wachsen (3) und es bildet sich ein gerader Riss durch die Probe (4). Die Mikrostruktur zeigt, dass ein duktiler Bruch vorliegt. Dabei schnürt sich die Probe ein und formt eine Schneide. Bei genauerer Betrachtung können sog. Lüdersbänder erkannt werden. Dies sind Rillen parallel zu Bruchkante. Nahe zur Risskante befinden sich bei beiden Proben kleine Löcher. Diese sind im Fall des unbeladenen Nickels in Linien angeordnet.

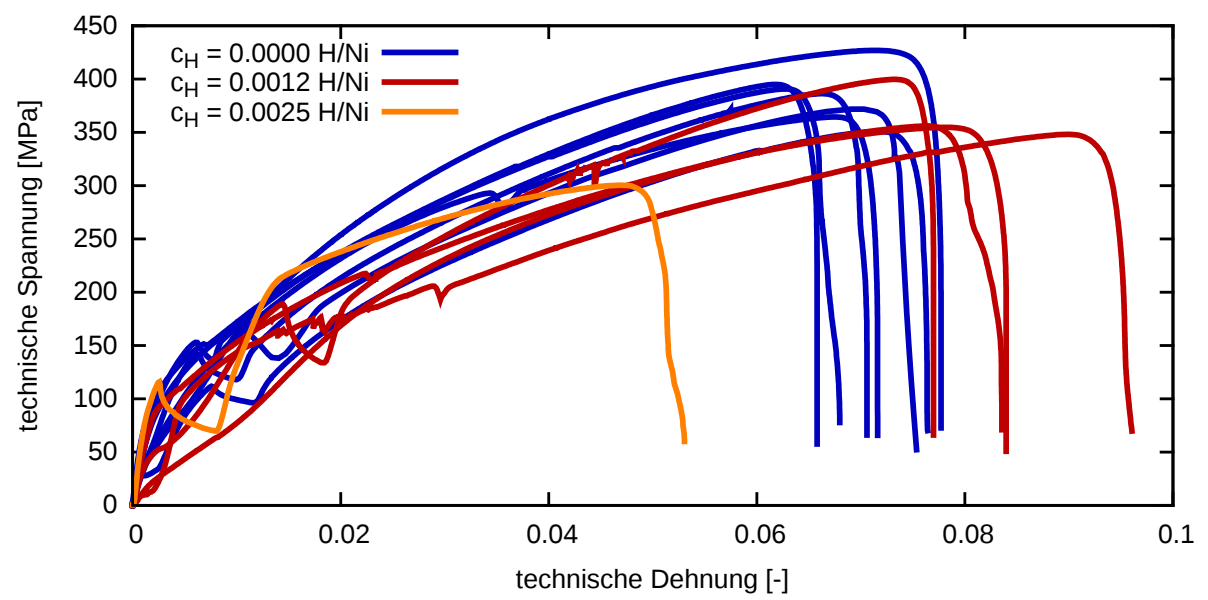

Abb. 80 Spannungs-Dehnungskurven von Nickel ohne Wasserstoff und mit einer Wasserstoffkonzentration von 0,0012 H/Ni bzw. 0,0025 H/Ni.

Bei mit 0,0012 H/Ni beladenen Zugproben ergibt sich eine ähnliche Mikrostruktur, vgl. Abbildung 82. Hier reißt die Probe auch durch die Bildung einer Schneide. Zusätzlich ist bei dieser Probe ein Übergang zu einem trichterförmigen Riss (rechte Seite) zu erkennen. Auf der Oberfläche sind wieder Hohlräume zu erkennen, welche in Linien angeordnet sind. Diese Löcher sind jedoch teilweise miteinander verbunden, was zur Ausbildung von kleinen Rissen führt. Die Ausschnitte der Mikroskop-Videoaufnahmen zeigen das bei diesem Bruch zunächst wieder einzelne Anrisse entstehen (1). Hier bilden sich jedoch zwei konkurrierende Risse aus, welche von beiden Seiten her zusammenlaufen. Der Riss, welcher von der linken Seite in die Probe hineinwächst, ist nicht so weit fortgeschritten wie der von der rechten Seite. D. h. die Einschnürung zur Schneide ist beim rechten Riss erst im Anfangsstadium, zum Zeitpunkt, wenn sich beide Risse in der Mitte treffen. Da die beiden Risse nicht auf gleicher Höhe sind und der rechte Riss die Probe schon vollkommen voneinander in zwei Teile getrennt hat, wird die linke Seite derart belastet, dass es zum Trichterbruch kommt. 

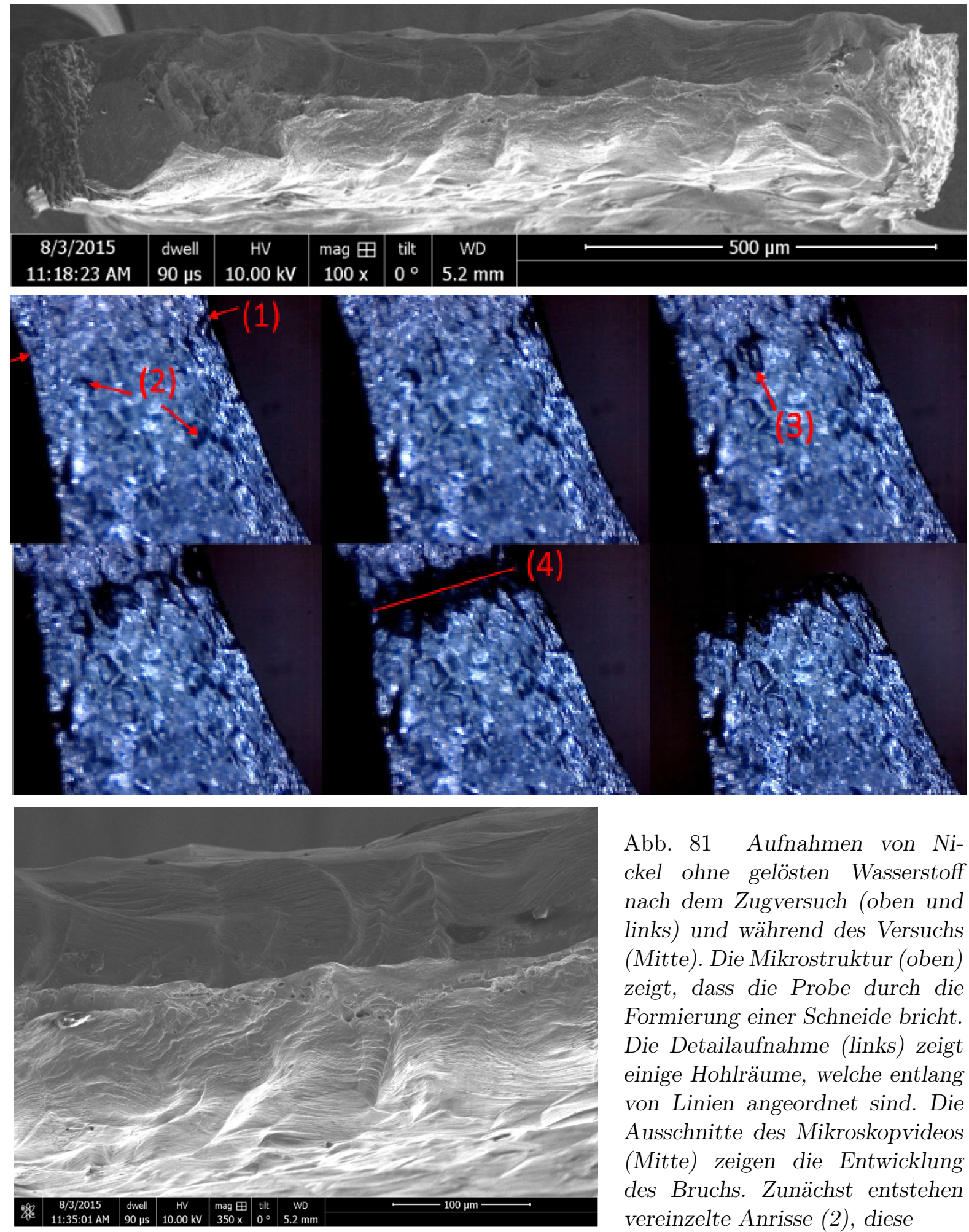

Abb. 81 Aufnahmen von $\mathrm{Ni}$ ckel ohne gelösten Wasserstoff nach dem Zugversuch (oben und links) und während des Versuchs (Mitte). Die Mikrostruktur (oben) zeigt, dass die Probe durch die Formierung einer Schneide bricht. Die Detailaufnahme (links) zeigt einige Hohlräume, welche entlang von Linien angeordnet sind. Die Ausschnitte des Mikroskopvideos (Mitte) zeigen die Entwicklung des Bruchs. Zunächst entstehen vereinzelte Anrisse (2), diese

wachsen (3) und werden letztendlich zum Riss (4), welcher nach und nach durch die Probe wandert. 

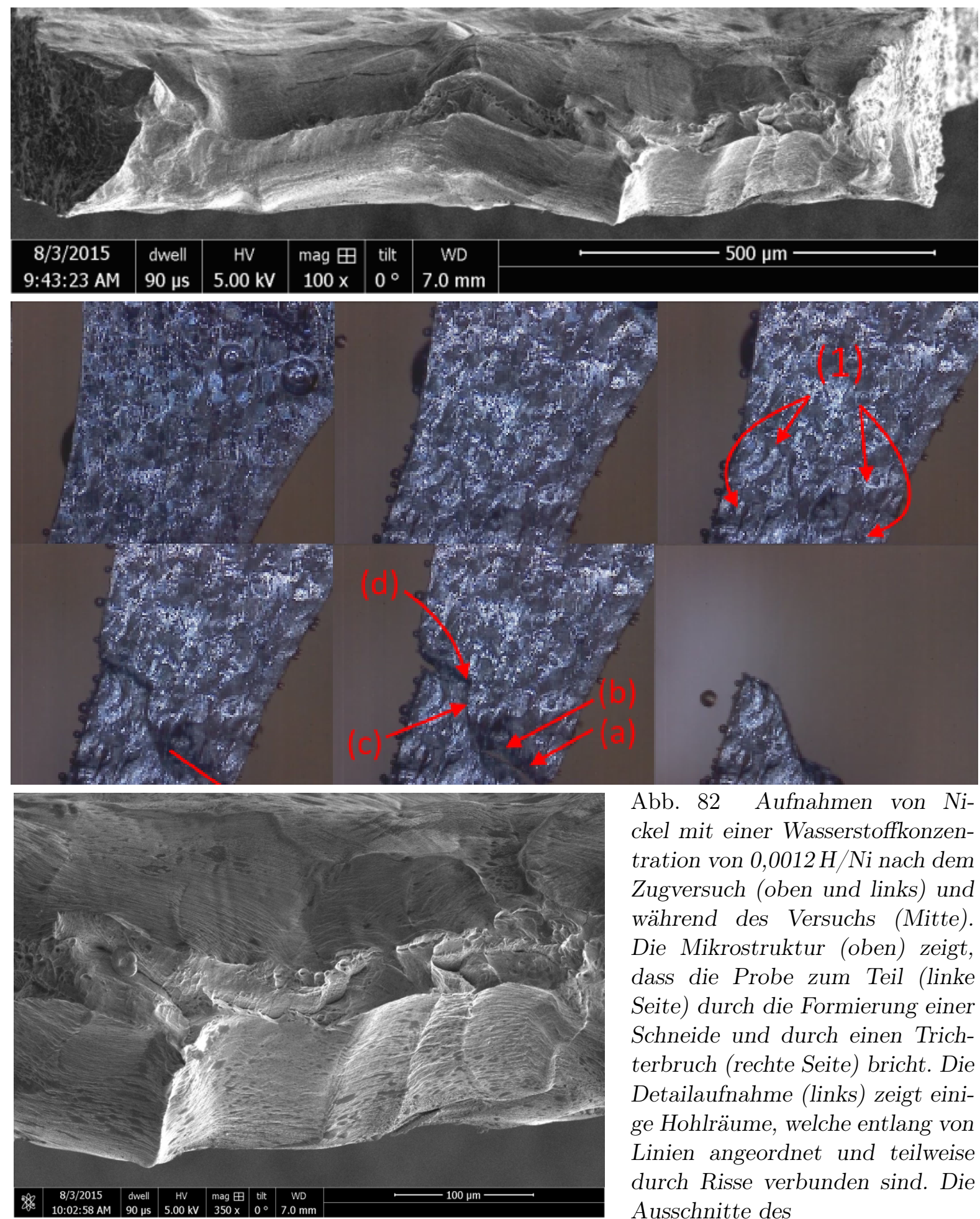

Abb. 82 Aufnahmen von $\mathrm{Ni}$ ckel mit einer Wasserstoffkonzentration von 0,0012 H/Ni nach dem Zugversuch (oben und links) und während des Versuchs (Mitte). Die Mikrostruktur (oben) zeigt, dass die Probe zum Teil (linke Seite) durch die Formierung einer Schneide und durch einen Trichterbruch (rechte Seite) bricht. Die Detailaufnahme (links) zeigt einige Hohlräume, welche entlang von Linien angeordnet und teilweise durch Risse verbunden sind. Die Ausschnitte des

Mikroskopvideos (Mitte) zeigen die Entwicklung des Bruchs. Zunächst entstehen vereinzelte Anrisse (1), dabei entstehen zwei konkurrierende Risse. Dabei ist der rechte weiter fortgeschritten und reißt als erstes (untere Reihe erstes Bild, rote Linie). In der Mitte treffen sich beide Risse (c) und (d). 


\section{Zugversuche an Vanadium:}

Die Mikrostruktur von Vanadium nach dem Zugversuch zeigt einen deutlichen Unterschied zwischen dem unbeladenen Zustand und dem mit einer Wassersstoffkonzentration von $0,03 \mathrm{H} / \mathrm{V}$. In Abbildung 83 ist die Bruchfäche einer unbeladenen Vanadiumprobe abgebildet. Bei dieser Probe hat sich die Querschnittsfläche derart eingeschnürt, dass eine scharfe Schneide zurückbleibt. Die Momentaufnahmen aus dem Mikroskopvideo zeigen, dass sich die Probe einschnürt und dann von einer Seite her durchreißt. In der Detailaufnahme ist in der Mitte eine unregelmäßigere Struktur zu erkennen. Es wird angenommen, dass bei diesem Versuch von beiden Seiten Risse entstanden sind, welche sich in der Mitte der Probe getroffen haben. Die Aufnahmen in Abbildung 84 zeigen Bruchflächen von zwei mit 0,03 H/V beladenen Proben. Durch die Zugabe von Wasserstoff reißen die Proben, indem diese sich zunächst etwas einschnüren. Bei der weiteren Rissentstehung kommt es entweder teilweise oder komplett zu einem spröden Spaltbruch. Wenn die Probe nur teilweise spröde reißt, reißt der andere Teil durch die Formierung einer Schneide, wie beim wasserstoffreien Vanadium.
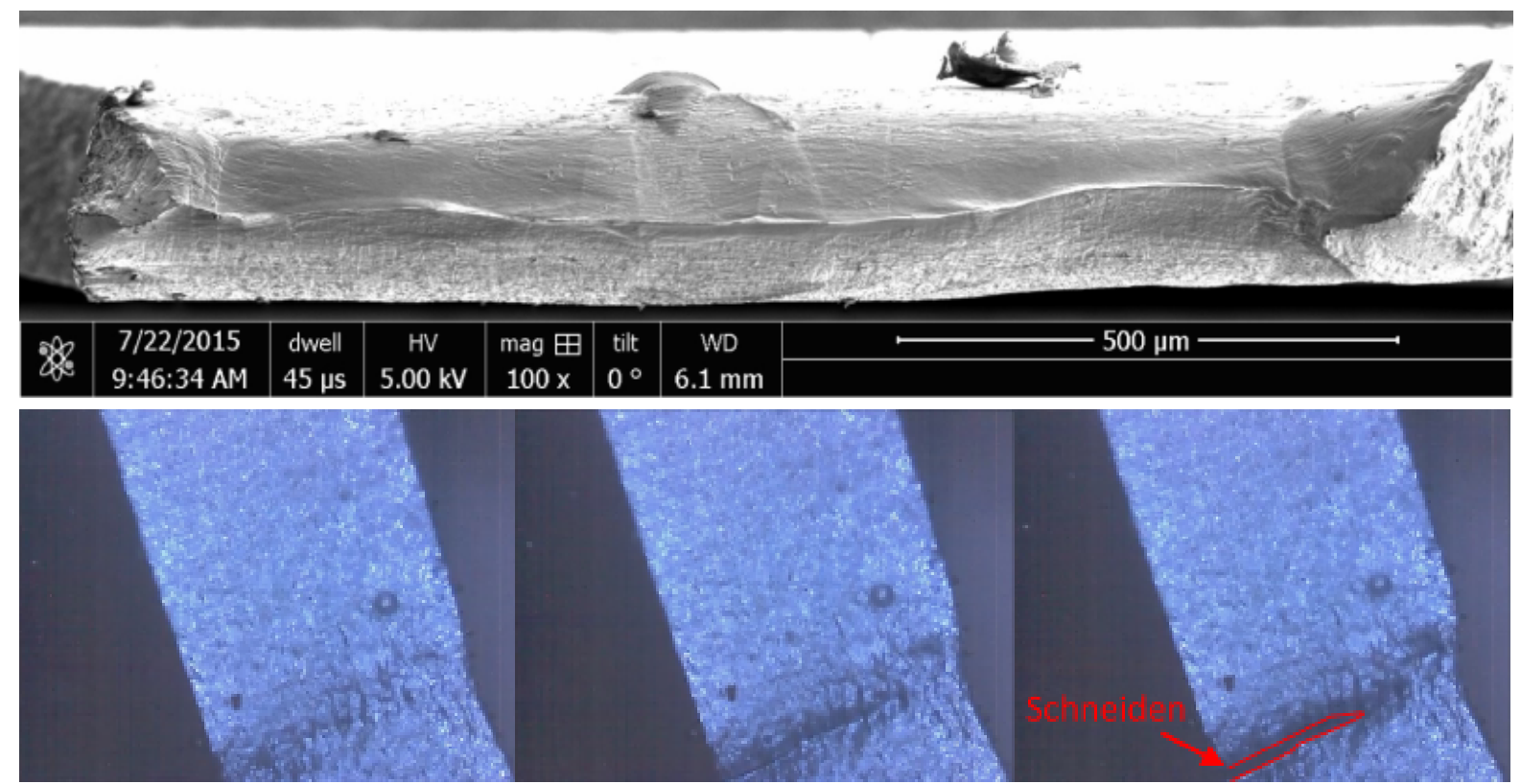

Abb. 83 Aufnahmen von Va-

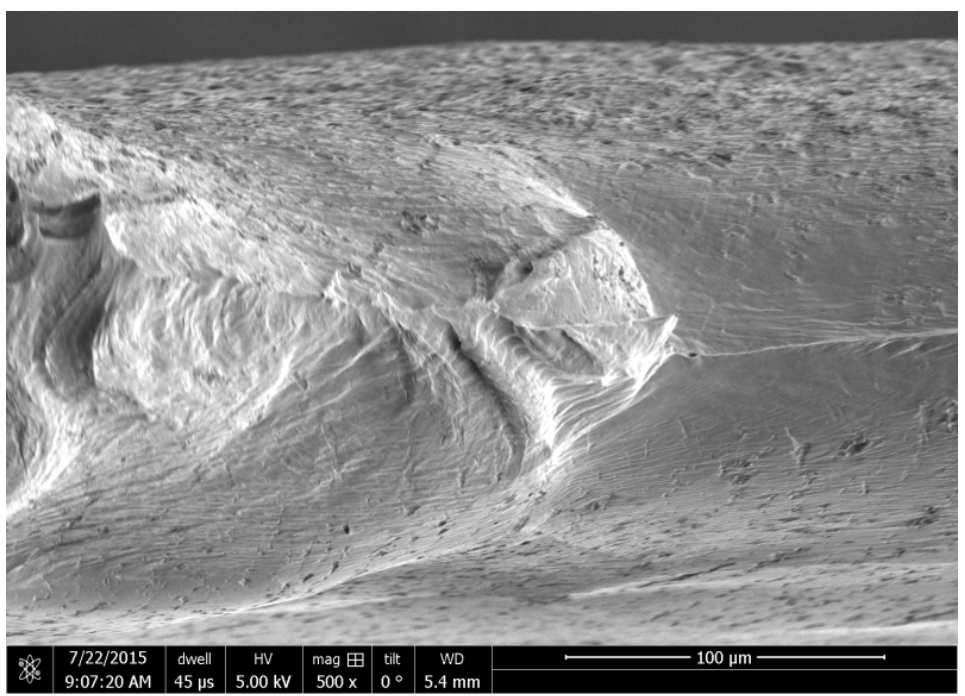
nadium ohne gelösten Wasserstoff. Im Übersichtsbild (oben) ist $\mathrm{zu}$ sehen, dass die Probe bei der Verformung eine Schneide ausgebildet hat. Die Verformung kann eindrucksvoll im Mikroskopvideo (Mitte) nachvollzogen werden. Die Probe schnürt sich zuerst ein und reißt dann von einer Seite komplett durch. Im Detailbild (links) ist eine andere Probe gezeigt, in der zwei Risse von beiden Seiten aufeinander zugewachsen sind. Die Unregelmäßigkeiten in der Mitte stammen von einem Versatz der Risse. 

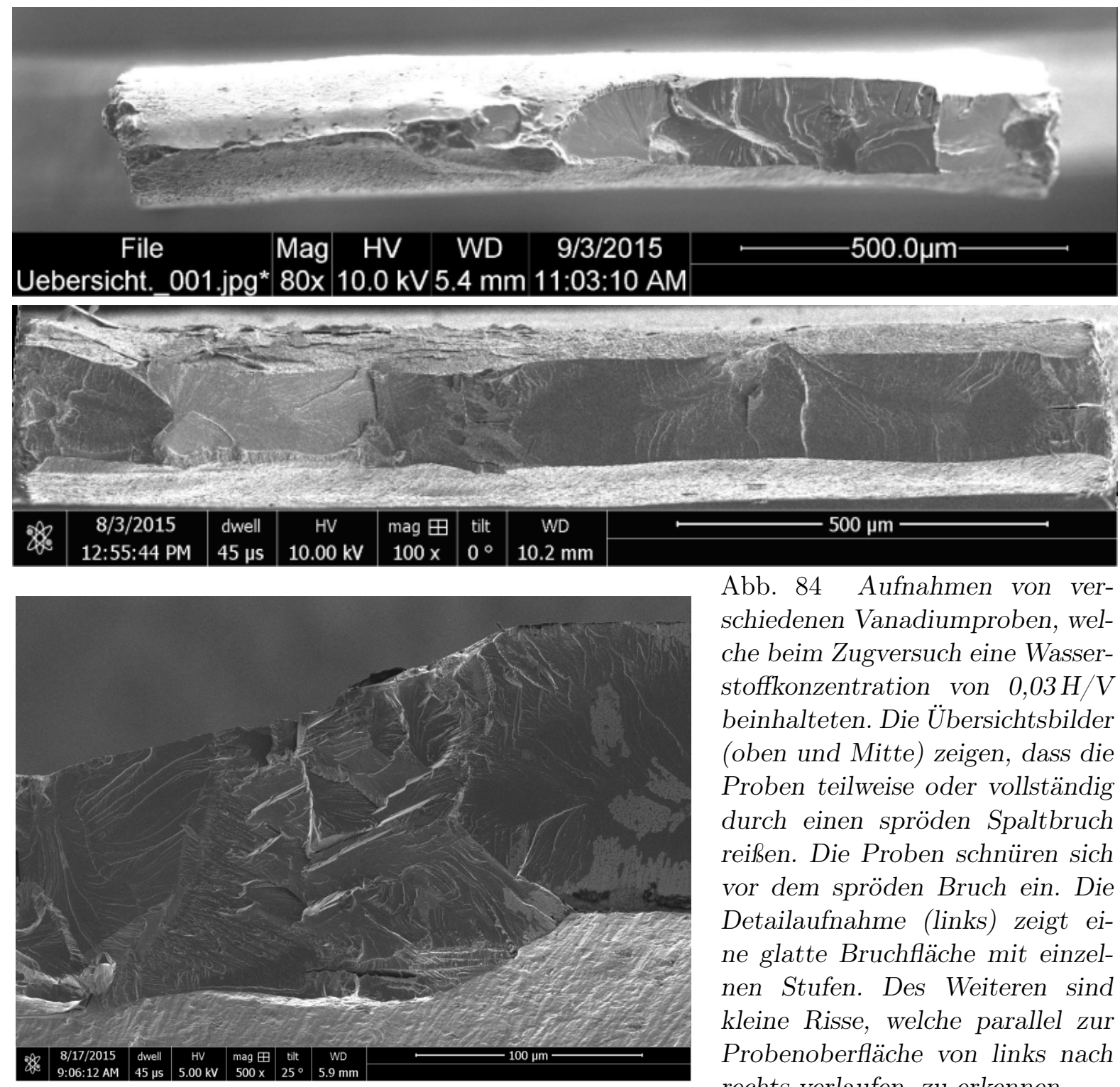

Abb. 84 Aufnahmen von verschiedenen Vanadiumproben, welche beim Zugversuch eine Wasserstoffkonzentration von $0,03 \mathrm{H} / \mathrm{V}$ beinhalteten. Die Übersichtsbilder (oben und Mitte) zeigen, dass die Proben teilweise oder vollständig durch einen spröden Spaltbruch reißen. Die Proben schnüren sich vor dem spröden Bruch ein. Die Detailaufnahme (links) zeigt eine glatte Bruchfläche mit einzelnen Stufen. Des Weiteren sind kleine Risse, welche parallel zur Probenoberfläche von links nach rechts verlaufen, zu erkennen. 


\section{Zugversuche an Tantal:}

Tantal weist den größten beobachteten Unterschied zwischen dem unbeladenen und dem mit 0,1 H/Ta beladenen Zustand auf. Ohne Wasserstoff reißt die Probe duktil, siehe Abbildung 86. Es sind viele Löcher auf der Bruchkante zu erkennen und die gesamte Probe hat sich eingeschnürt. Wenn Wasserstoff sich in der Probe befindet (vgl. Abbildung 87), kommt es zu einem vollständigen spröden Bruch. Dabei entstehen sehr glatte Bruchkanten und die Probe zeigt keine Einschnürungen mehr. Bei näherer Betrachtung ist zu erkennen, dass sich auf der Oberfläche auch Späne ablösen können.

In den Spannungs-Dehnungskurven (Abbildung 85) ist zu erkennen, dass die Proben ohne Wasserstoff einem typischen duktilen Verlauf folgen: Auf einen linear elastischen Abschnitt folgt ein Verfestigungsbereich und anschließend kommt es zu einem Abfall aufgrund der Einschnürung. Die Proben mit Wasserstoff zeigen nur den linear elastischen Bereich und reißen schon in diesem Bereich. Die Abweichungen vom idealen Verhalten sind auf die Verwendung der Plexiglasstäbe zurückzuführen.

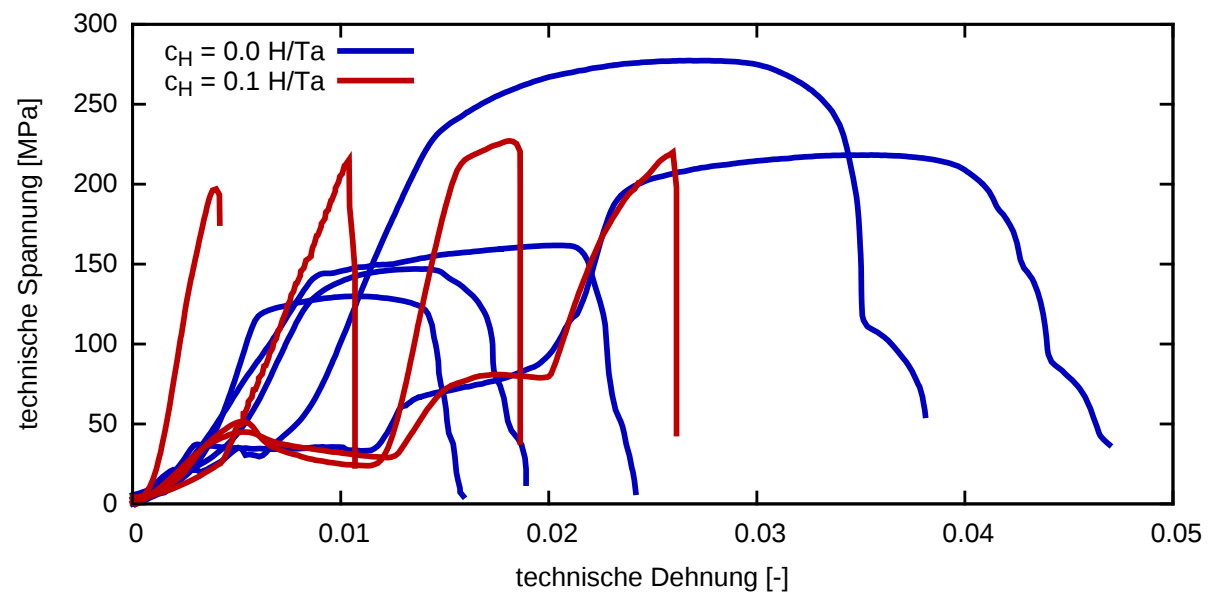

Abb. 85 Spannungs-Dehnungskurven von Tantal ohne Wasserstoff und mit einer Wasserstoffkonzentration von $0,1 \mathrm{H} / \mathrm{Ta}$. 

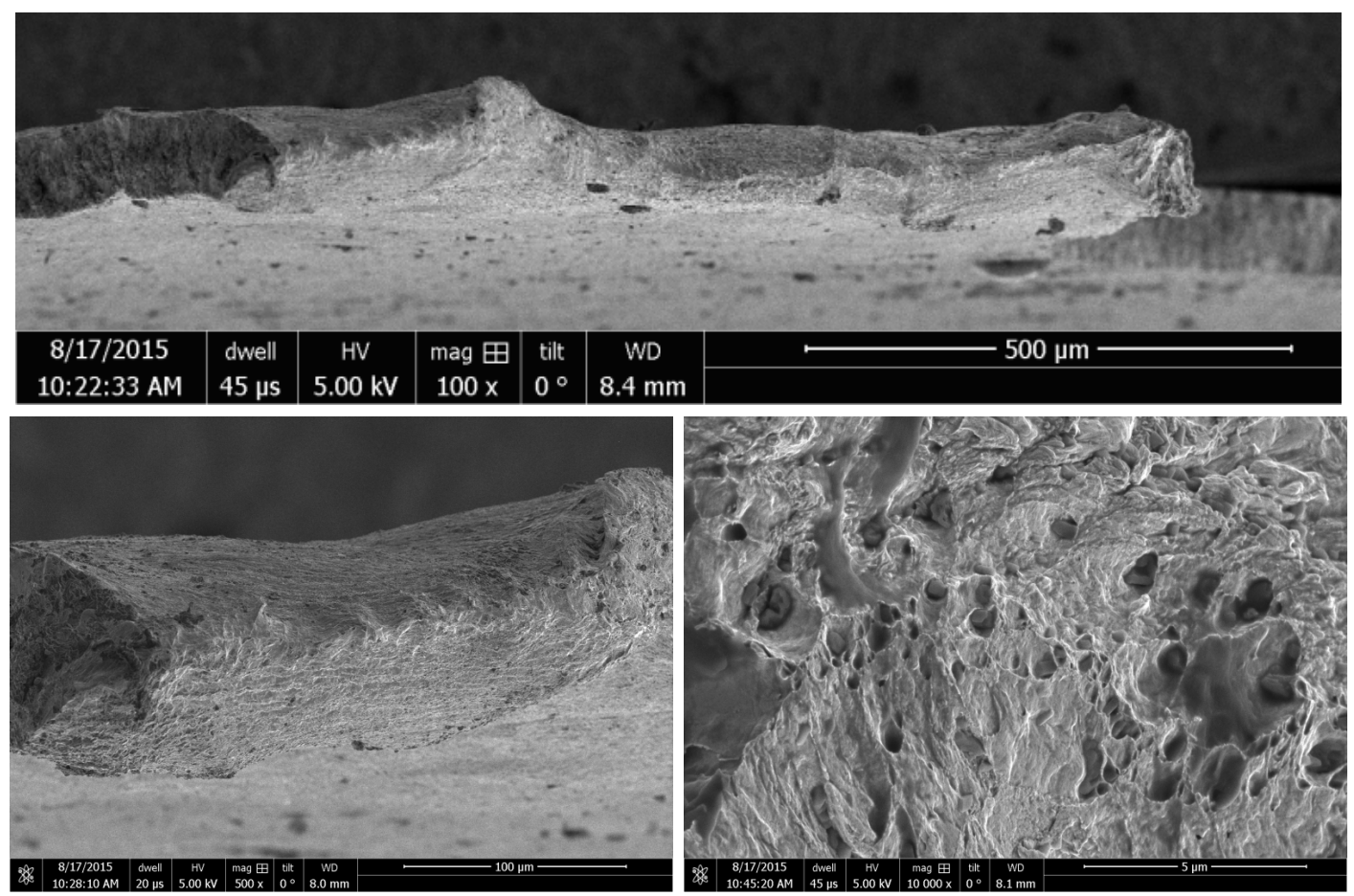

Abb. 86 Aufnahmen von Tantal ohne gelösten Wasserstoff nach dem Zugversuch. Im Übersichtsbild (oben) ist zu erkennen, dass sich die Probe beim Ziehen einschnürt, es bildet sich eine abgerundete Bruchkante aus. Im Detailbild (links unten) sind mehrere Stufen in der Einschnürung zu sehen und es ist in dem gezeigten Ausschnitt kein klarer Übergang zwischen unverformtem Bereich und Bruchkante zu erkennen. Bei noch höherer Vergrößerung (rechts unten) zeigt sich, dass die Oberfläche von vielen Löchern übersät ist, welche keiner Regelmäßigkeit folgen.
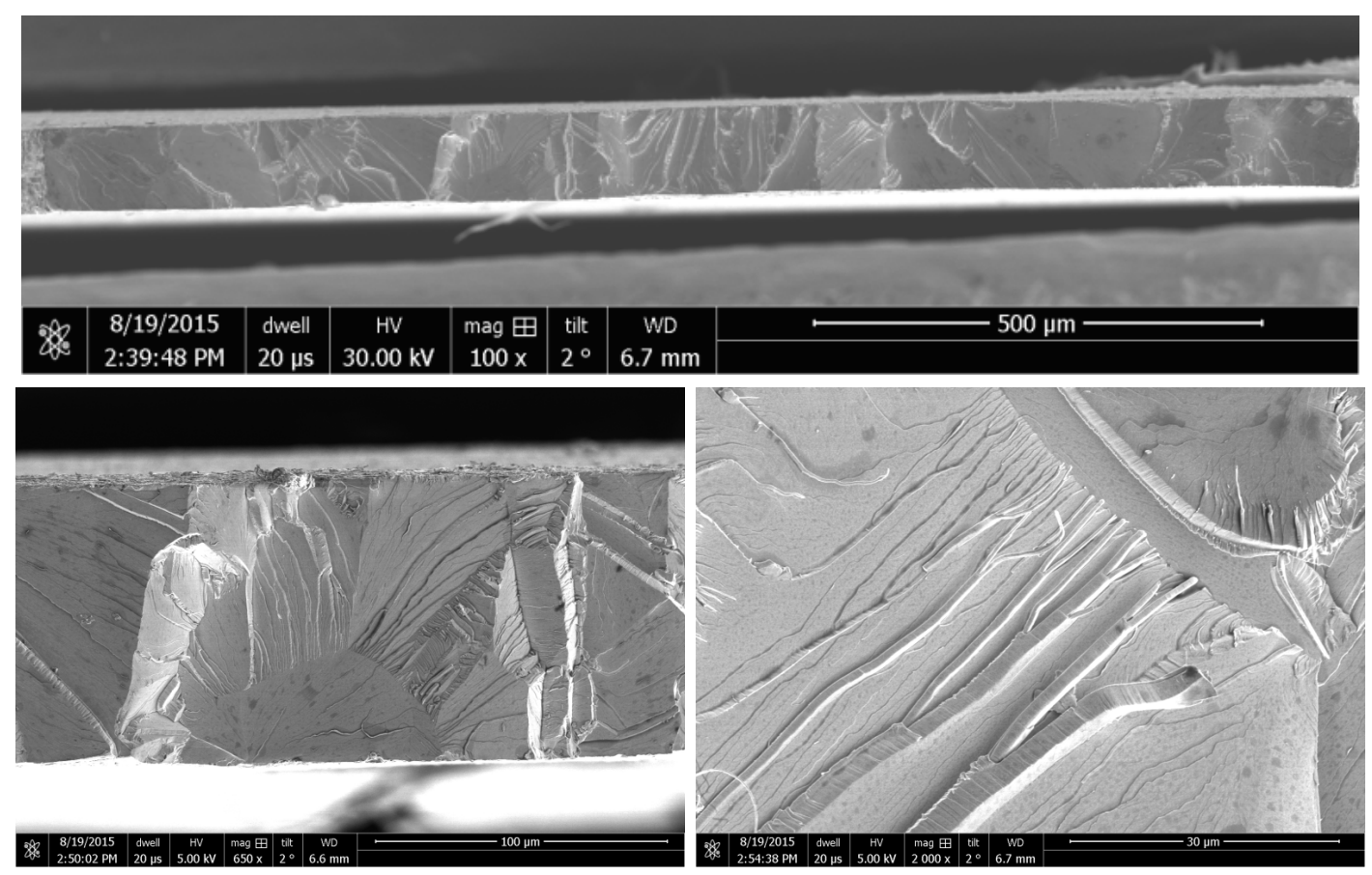

Abb. 87 Aufnahmen nach dem Zugversuch an Tantal mit einer Wasserstoffkonzentration von $0,1 \mathrm{H} /$ Ta. Bei Anwesenheit von Wasserstoff verändert sich das Bruchverhalten vollkommen im Vergleich zu unbeladenen Proben. Im Übersichtsbild (oben) ist zu erkennen, dass die Probe vollständig spröde gerissen ist. Außerdem ist keine Einschnürung zu erkennen. In der Detailaufnahme (links unten) zeigt sich, dass der Bruch auf der Bildung von glatten Flächen beruht, welche durch Stufen voneinander getrennt sind. Bei höherer Vergrößerung (rechts unten) zeigt sich zusätzlich, dass sich auch Späne ablösen können. 


\subsection{Veröffentlichungen}

\section{Peer-review Veröffentlichungen:}

[1] C. Borchers, Y. Chen, M. Deutges, S. Goto, R. Kirchheim. Carbon-defect interaction during recovery and recrystallization of heavily deformed pearlitic steel wires. Phil. Mag. Lett., 90:581-588, 2010

[2] M. Deutges, I. Knorr, C. Borchers, C.A. Volkert, R. Kirchheim. Influence of hydrogen on the deformation morphology of vanadium $\left(\begin{array}{lll}1 & 0 & 0\end{array}\right)$ micropillars in the $\alpha$-phase of the vanadium-hydrogen system. Scripta Mater., 68:71-74, 2013

[3] Y.Z. Chen, H.P. Barth, M. Deutges, C. Borchers F. Liu, R. Kirchheim. Increase in dislocation density in cold-deformed $\mathrm{Pd}$ using $\mathrm{H}$ as a temporary alloying addition. Scripta Mater., 68:743-746, 2013

[4] C. Borchers, A. Lehmberg, M. Deutges, J. Ciẑek, R. Kirchheim. Effect of annealing on point defect population in cold-drawn pearlitic steel wires. Scripta Mater., 86 17-19, 2014

[5] M. Deutges, H.P. Barth, Y. Chen, C. Borchers, R. Kirchheim. Hydrogen diffusivities as a measure of relative dislocation densities in palladium and increase of the density by plastic deformation in the presence of dissolved hydrogen. Acta Mater., 82:266-274, 2015

[6] C. Borchers, C. Garve, M. Tiegel, M. Deutges, A. Herz, K. Edalati, R. Pippan, Z. Horita, R. Kirchheim. Nanocrystalline steel obtained by mechanical alloying of iron and graphite subsequently compacted by high-pressure torsion Acta Mater., 97:207-215, 2015

[7] C. Borchers, M. Lenz, M. Deutges, H. Klein, F. Gärtner, M. Hammerschmidt, H. Kreye. Microstructure and mechanical properties of medium-carbon steel bonded on low-carbon steel by explosive welding Mater. Design, 89:369-376, 2016

[8] M.C. Tiegel, M.L. Martin, A.K. Lehmberg, M. Deutges, C. Borchers, R. Kirchheim. Crack and blister initiation and growth due to hydrogen loading in purified iron Eingereicht bei Acta Mater., 2016

\section{Konferenzbeiträge:}

[1] M. Deutges, I. Knorr, H.P. Barth, Y. Chen, C. Borchers, C. Volkert, R. Kirchheim. Influence of hydrogen on the defect structure in metals subjected to plastic deformation. DPG-Frühjahrstagung, Regensburg 2013

[2] M. Deutges, C. Borchers, R. Kirchheim. The influence of dissolved hydrogen in palladium on the pop-in load. DPG-Frühjahrstagung, Dresden 2014

[3] M. Deutges, C. Borchers, R. Kirchheim. In-situ Nanoindentation of Palladium-Hydrogen Alloys. 14th International Symposium on Metal-Hydrogen Systems, Manchester 2014

[4] M. Deutges, H.P. Barth, Y. Chen, C. Borchers, R. Kirchheim. Hydrogen diffusivity as a measure for relative dislocation densities in palladium. DPG-Frühjahrstagung, Berlin 2015 


\section{Literatur}

[1] H.C. Rogers. Hydrogen Embrittlement of Metals. Science, 159:1057-1064, 1968.

[2] S.-J. Kim, S.-K. Jang, J.-I. Kim. Electrochemical Study of Hydrogen Embrittlement and Optimum Cathodic Protection Potential of Welded High Strength Steel. Met. Mater. Int., 11:63-69, 2005.

[3] J. Ćwiek. Hydrogen degradation of high-strength steels. J. Achie. Mater. Manu. Eng., 37: 193-212, 2009.

[4] H. Uyama, H. Yamada, H. Hidaka, N. Mitamura. The Effects of Hydrogen on Microstructural Change and Surface Originated Flaking in Rolling Contact Fatigue. Tribol. Online, 6: 123-132, 2011.

[5] M. Kohara, T. Kawamura, M. Egami. Study on Mechanism of Hydrogen Generation from Lubricants. Tribol. T., 49:53-60, 2006.

[6] L.T. Popoola, A.S. Grema, G.K. Latinwo, B. Gutti, A.S. Balogun. Corrosion problems during oil and gas production and its mitigation. Int. J. Ind. Chem., 4:35-50, 2013.

[7] S.-J. Kim, M. Okido, K.-M. Moon. An Electrochemical Study of Cathodic Protection of Steel Used for Marine Structures. Korean J. Chem. Eng., 20:560-565, 2003.

[8] J. Billingham, J.V. Sharp, J. Spurrier, P.J. Kilgallon. Review of the performance of high strength steels used offshore. Health and Safety Executive (HSE), 2003.

[9] H. Tanimoto, H. Tanaka, J. Sugimura. Obersavation of Hydrogen Permeation into Fresh Bearing Steel Surface by Thermal Desorption Spectroscopy. Tribol. Online, 6:291-296, 2011.

[10] M.K. Amosa, I.A. Mohammed, S.A. Yaro. Sulphide Scavengers in Oil and Gas Industry A Review. Nafta, 61:85-92, 2010.

[11] I.V. Babich, J.A. Moulijn. Science and technology of novel processes for deep desulfurization of oil refinery streams: a review. Fuel, 82:607-631, 2003.

[12] P. Cotterill. The hydrogen embrittlement of metals. Prog. Met. Phys., 9:205-301, 1961.

[13] S. P. Lynch. Hydrogen embrittlement and liquid-metal embrittlement in nickel single crystals. Scripta Metall. Mater., 13:1051-1056, 1979.

[14] H. Vehoff, W. Rothe. Gaseous hydrogen embrittlement in FeSi- and Ni-single crystals. Acta Metall. Mater., 31:1781-1793, 1983.

[15] S. Gahr, M.L. Grossbeck, H.K. Birnbaum. Hydrogen embrittlement of Nb I - Macroscopic behavior at low temperatures. Acta Metall. Mater., 25:125-134, 1977.

[16] M.L. Grossbeck, H.K. Birnbaum. Low temperature hydrogen embrittlement of Nb II Microscopic observations. Acta Metall. Mater., 25:135-147, 1977.

[17] D.P. Escobar, C. Miñambres, L. Duprez, K. Verbeken, M. Verhaege. Internal and surface damage of multiphase steels and pure iron after electrochemical hydrogen charging. Corros. Sci., 53:3166-3176, 2011.

[18] M.C. Tiegel, M.L. Martin, A.K. Lehmberg, M. Deutges, C. Borchers, R. Kirchheim. Crack and blister initiation and growth due to hydrogen loading in purified iron. Acta Mater., In Press., 2016. 
[19] S.M. Myers, M.I. Baskes, H.K. Birnbaum, J.W. Corbett, G.G. DeLeo, S.K. Estreicher, E.E. Mailer, P. Jena, N.M. Johnson, R. Kirchheim, S.J. Pearton, M.J. Stavola. Hydrogen interactions with defects in crystalline solids. Rev. Mod. Phys., 64:559-617, 1992.

[20] S. Lynch. Hydrogen embrittlement phenomena and mechanisms. Corros. Rev., 30:105-123, 2012.

[21] H.K. Birnbaum, P. Sofronis. Hydrogen-enhanced localized plasticity - a mechanism for hydrogen-related fracture. Mater. Sci. Eng., A176:191-202, 1994.

[22] H.K. Birnbaum. Hydrogen Effects on Deformation and Fracture: Science and Sociology. MRS Bulletin, 28:479-485, 2003.

[23] I.M. Robertson, P. Sofronis, A. Nagao, M.L. Martin, S. Wang, D.W. Gross, K.E. Nygren. Hydrogen Embrittlement Understood. Metall. Mater. Trans. B, 46:1085-1103, 2015.

[24] P. Rozenak, I.M. Robertson, H.K. Birnbaum. HVEM studies of the effects of hydrogen on the deformation and fracture of AISI type 316 austenitic stainless steel. Acta Mater., 38: 2031-2040, 1990.

[25] P.J. Ferreira, I.M. Robertson, H.K. Birnbaum. Hydrogen effects on the interaction between dislocations. Acta Mater., 46:1749-1757, 1998.

[26] P.J. Ferreira, I.M. Robertson, H.K. Birnbaum. Hydrogen effects on the character of dislocations in high-purity aluminum. Acta Mater., 47:2991-2998, 1999.

[27] R. Kirchheim. Reducing grain boundary, dislocation line and vacancy formation energies by solute segregation. I. Theoretical background. Acta Mater., 55:5129-5138, 2007.

[28] R. Kirchheim. Reducing grain boundary, dislocation line and vacancy formation energies by solute segregation II. Experimental evidence and consequences. Acta Mater., 55:5139-5148, 2007.

[29] R. Kirchheim. On the solute-defect interaction in the framework of a defactant concept. Int. J. Mat. Res., 100:483-487, 2009.

[30] R. Kirchheim. Revisiting hydrogen embrittlement models and hydrogen-induced homogeneous nucleation of dislocations. Scripta Mater., 62:67-70, 2010.

[31] S. Goto, R. Kirchheim, T. Al-Kassab, C. Borchers. Application of cold drawn lamellar microstructure for developing ultra-high strength wires. T. Nonferr. Metal. Soc., 17:1129$1138,2007$.

[32] C. Borchers, Y. Chen, M. Deutges, S. Goto, R. Kirchheim. Carbon-defect interaction during recovery and recrystallization of heavily deformed pearlitic steel wires. Phil. Mag. Lett., 90: $581-588,2010$.

[33] Y. Li, D. Raabe, M. Herbig, P.-P. Choi, S. Goto, A. Kostka, H. Yarita, C. Borchers, R. Kirchheim. Segregation Stabilizes Nanocrystalline Bulk Steel with Near Theoretical Strength. Phys. Rev. Lett., 113:106104, 2014.

[34] G.K. Williamson, R.E. Smallman. III. Dislocation densities in some annealed and coldworked metals from measurements on the X-ray debye-scherrer spectrum. Phil. Mag., 1: 34-46, 1956.

[35] E. Ahmad, F. Karim, K. Saeed, T. Manzoor, G.H. Zahid. Effect of cold rolling and annealing on the grain refinement of low alloy steel. Mater. Sci. Eng., 60:012029, 2014. 
[36] J. Gubicza, Z. Hegedüs, J.L. Lábár, A. Kauffmann, J. Freudenberger, V. Subramanya Sarma. Solute redistribution during annealing of a cold rolled $\mathrm{Cu}-\mathrm{Ag}$ alloy. J. Alloy Compd., 623: 96-103, 2015.

[37] Y.Z. Chen, A. Herz, Y.J. Li, C. Borchers, P. Choi, D. Raabe, R. Kirchheim. Nanocrystalline Fe-C alloys produced by ball milling of iron and graphite. Acta Mater., 61:3172-3185, 2013.

[38] Y. Fukai. The Metal-Hydrogen System. Springer-Verlag Berlin Heidelberg, 2005.

[39] P. Vajeeston, P. Ravindran, R. Vidya, A. Kjekshus, H. Fjellvåg. Site preference of hydrogen in metal, alloy, and intermetallic frameworks. Europhys. Lett., 72:569-575, 2005.

[40] M. Deutges, H.P. Barth, Y. Chen, C. Borchers, R. Kirchheim. Hydrogen diffusivities as a measure of relative dislocation densities in palladium and increase of the density by plastic deformation in the presence of dissolved hydrogen. Acta Mater., 82:266-274, 2015.

[41] C.E. Buckley, H.K. Birnbaum, J.S. Lin, S. Spooner, D. Bellmann, P. Staron, T.J. Udovic, E. Hollar. Characterization of $\mathrm{H}$ defects in the aluminium-hydrogen system using smallangle scattering techniques. J. Appl. Crystallogr., 34:119-129, 2001.

[42] H. Suzuki, D. Kobayashi, N. Hanada, K. Takai, Y. Hagihara. Existing state of hydrogen in electrochemically charged commercial-purity aluminum and its effects on tensile properties. Mater. Trans., 52:1741-1747, 2011.

[43] Y.Z. Chen, H.P. Barth, M. Deutges, C. Borchers, F. Liu, R. Kirchheim. Increase in dislocation density in cold-deformed Pd using $\mathrm{H}$ as a temporary alloying addition. Scripta Mater., 68:743-746, 2013.

[44] M. Deutges, I. Knorr, C. Borchers, C.A. Volkert, R. Kirchheim. Influence of hydrogen on the deformation morphology of vanadium $\left(\begin{array}{lll}1 & 0 & 0\end{array}\right)$ micropillars in the $\alpha$-phase of the vanadium-hydrogen system. Scripta Mater., 68:71-74, 2013.

[45] G. Gottstein. Physikalische Grundlagen der Materialkunde. Springer-Verlag Berlin Heidelberg, 2007.

[46] P. Haasen. Physikalische Metallkunde. Springer-Verlag Berling Heidelberg GmbH, 1994.

[47] Y. Fukai, Y. Ishii, Y. Goto, K. Watanabe. Formation of superabundant vacancies in Pd-H alloys. J. Alloy Compd., 313:121-132, 2000.

[48] M. Krystian, D. Setman, , B. Mingler, G. Krexner, M.J. Zehetbauer. Formation of superabundant vacancies in nano-Pd-H generated by high-pressure torsion. Scripta Mater., 62: 49-52, 2010.

[49] C. Borchers, A. Lehmberg, M. Deutges, J. Čížek, R. Kirchheim. Effect of annealing on point defect population in cold-drawn pearlitic steel wires. Scripta Mater., 86:17-19, 2014.

[50] R.E. Smallman, A.H.W. Ngan. Physical Metallurgy and Advanced Materials. Elsevier Ltd., 2007.

[51] D. Hull, D.J. Bacon. Introduction to Dislocations. Elsevier Ltd., 2011.

[52] J.A. Rayne. Elastic Constants of Palladium from 4.2-300K. Phys. Rev., 118:1545-1549, 1960.

[53] F.D. Manchester, A. San-Martin, J.M. Pitre. The H-Pd (Hydrogen-Palladium) System. J. Phase. Equilib., 15:62-83, 1994.

[54] J.P. Hirth, J. Lothe. Theory of Dislocations. McGraw-Hill, Inc., 1968. 
[55] F. Louchet, L.P. Kubin. Dislocation Processes in B.C.C. Metals. Phys. Status. Solidi. A, 56:169-176, 1979.

[56] M.S. Duesbery, V. Vitek. Plastic anisotropy in b.c.c. transition metals. Acta Mater., 46: 1481-1492, 1998.

[57] L.P. Kubin, F. Louchet. Description of low-temperature interstitial hardening of the b.c.c. lattice from in situ experiments. Phil. Mag., 38:205-221, 1978.

[58] P.A. Gordon, T. Neeraj, Y. Li, J. Li. Screw dislocation mobility in BCC metals: the role of the compact core on double-kink nucleation. Modelling Simul. Mater. Sci. Eng., 18:085008, 2010 .

[59] T.D. Swinburne, S.L. Dudarev, S.P. Fitzgerald, M.R. Gilbert, A.P. Sutton. Theory and simulation of the diffusion of kinks on dislocations in bcc metals. Phys. Rev. B, 87:064108, 2013.

[60] F. Louchet, L.P. Kubin, D. Vesely. In situ deformation of b.c.c. crystals at low temperatures in a high-voltage electron microscope Dislocation mechanisms and strain-rate equation. Phil. Mag., 39:433-454, 1979.

[61] R. Kirchheim. Interaction of hydrogen with dislocations in palladium - I. Activity and diffusivity and their phenomenological interpretation. Acta Metall. Mater., 29:835-843, 1981.

[62] M. Maxelon, A. Pundt, W. Pyckhout-Hintzen, J. Barker, R. Kirchheim. Interaction of hydrogen and deuterium with dislocations in palladium as observed by small angle neutron scattering. Acta Mater., 49:2625-2634, 2001.

[63] M. Maxelon, A. Pundt, W. Pyckhout-Hintzen, R. Kirchheim. Small angle neutron scattering of hydrogen segregation at dislocations in palladium. Scripta Mater., 44:817-822, 2001.

[64] R. Kirchheim. Solid solution softening and hardening by mobile solute atoms with special focus on hydrogen. Scripta Mater., 67:767-770, 2012.

[65] D.R. Trinkle, C. Woodward. The Chemistry of Deformation: How Solutes Soften Pure Metals. Science, 310:1665-1667, 2005.

[66] G. Alefeld, J. Völkl. Hydrogen in metals I. Springer-Verlag Berlin Heidelberg New York, 1978.

[67] H. Inui, T. Yamamoto, M. Hirota, M. Yamaguchi. Lattice defects introduced during hydrogen absorption-desorption cycles and their effects on P-C characteristics in some intermetallic compounds. J. Alloy Compd., 330-332:117-124, 2002.

[68] Y. Ozu, T. Kuji, H.-H. Uchida. Pulverization behavior of $\mathrm{LaNi}_{5}$ with alkaline pretreatment. J. Alloy Compd., 330-332:632-635, 2002.

[69] H.D. Carstanjen. Interstitial Positions and Vibrational Amplitudes of Hydrogen in Metals Investigated by Fast Ion Channeling. Phys. Status. Solidi. A, 59:11-26, 1980.

[70] C. Kittel. Einführung in die Festkörperphysik. Oldenbourg Wissenschaftsverlag, 2006.

[71] R. Griessen, A. Driessen. Heat of formation and band structure of binary and ternary metal hydrides. Phys. Rev. B, 30:4372-4381, 1984.

[72] H. Hemmes, A. Driessen, R. Griessen, M. Gupta. Isotope effects and pressure dependence of the T, of superconducting stoichiometric PdH and PdD synthesized and measured in a diamond anvil cell. Phys. Rev. B, 39:4110-4118, 1989. 
[73] R. Kirchheim, A. Pundt. Hydrogen in Metals. In: D.E. Laughlin, K. Hono (Eds.), Physical Metallurgy, 2597-2705. 2014.

[74] A. Sieverts. Die Aufnahme von Gasen durch Metalle. Z. Metallkd., 21:37-46, 1929.

[75] J.O'M. Bockris, J. McBreen, L. Nanis. The Hydrogen Evolution Kinetics and Hydrogen Entry into $\alpha$-Iron. J. Electrochem. Soc., 112:1025-1031, 1965.

[76] R.J. Behm, V. Penka, M.-G. Cattania, K. Christmann, G. Ertl. Evidence for "subsurface" hydrogen on $\operatorname{Pd}(110)$ : An intermediate between chemisorbed and dissolved species. J. Chem. Phys., 78:7486-7490, 1983.

[77] C. Borchers, T. Michler, A. Pundt. Effectof Hydrogen on the Mechanical Properties of Stainless Steels. Adv. Eng. Mater., 10:11-23, 2008.

[78] A. Remhof, A. Borgschulte. Thin-Film Metal Hydrides. Chem. Phys. Chem., 9:2440-2455, 2008.

[79] A. Züttel. Metall-Hydride. Vorlesungsskript, 2005.

[80] A. Barnoush. Hydrogen embrittlement, revisited by in situ electrochemical nanoindentation. Dissertation, Universität des Saarlandes, 2007.

[81] C.E. Mortimer, U. Müller, J. Beck. Chemie: Das Basiswissen der Chemie. Georg Thieme Verlag, 2014. 11. Auflage.

[82] R. Kirchheim. Interaction of hydrogen with external stress fields. Acta Metall. Mater., 34: 37-42, 1986.

[83] American society for metals. Diffusion. Chapman \& Hall LTD, 1973.

[84] C. Kittel, H. Kroemer. Thermal Physics. W. H. Freeman, 1980.

[85] R.B. De Boer. On the thermodynamics of pressure solution-interaction between chemical and mechanical forces. Geochim. Cosmochim. Ac., 41:249-256, 1977.

[86] A.H. Cottrell, B.A. Bilby. Dislocation Theory of Yielding and Strain Ageing of Iron. Proc. Phys. Soc. A, 62:49-62, 1949.

[87] D. Chatain, P. Wynblatt, G.S. Rohrer. Equilibrium crystal shape of Bi-saturated Cu crystals at 1223 K. Acta Mater., 53:4057-4064, 2005.

[88] A. Pundt, R. Kirchheim. Hydrogen in Metals: Microstructural Aspects. Annu. Rev. Mater. Res., 36:555-608, 2006.

[89] A. Pundt. Nanoskalige Metall-Wasserstoff-Systeme. Habilitationsschrift, Georg-AugustUniversität Göttingen, 2005.

[90] S.M. Solov'ev, C. Pettenkofer, I.I. Pronin, N.D. Potekhina, V.N. Petrov. Interaction of Pd electron states with adsorbed hydrogen. Surf. Science, 608:165-172, 2013.

[91] S.B. Gesari, M.E. Pronsato, A. Juan. Simulation of hydrogen trapping at defects in Pd. Int. J. Hydrogen Energ., 34:3511-3518, 2009.

[92] Y. Tateyama, T. Ohno. Atomic-scale Effects of Hydrogen in Iron toward Hydrogen Embrittlement: Ab-initio Study. ISIJ Int., 43:573-578, 2003.

[93] A.T. Paxton. From quantum mechanics to physical metallurgy of steels. Mater. Sci. Tech. Ser., 30:1063-1070, 2014. 
[94] J.W. Gibbs. The collected works of J. Willard Gibbs. Trans. Conn. Acad., 3:108-248, 1876. also in "The collected work of J. W. Gibbs", Vol. I, Longmans, Green \& Co., New York, 1928 , p. 55.

[95] E. Tal-Gutelmacher, R. Gemma, C.A. Volkert, R. Kirchheim. Hydrogen effect on dislocation nucleation in a vanadium (1 000$)$ single crystal as observed during nanoindentation. Scripta Mater., 63:1032-1035, 2010.

[96] M. Wen, Z. Li, , A. Barnoush. Atomistic Study of Hydrogen Effect on Dislocation Nucleation at Crack Tip. Adv. Eng. Mater., 15:1146-1151, 2013.

[97] R. Matsumoto, S. Seki, S. Taketomi, N. Miyazaki. Hydrogen-related phenomena due to decreases in lattice defect energies-Molecular dynamics simulations using the embedded atom method potential with pseudo-hydrogen effects. Comp. Mater. Sci., 92:362-371, 2014.

[98] J.-H. Shim, W.-S. Ko, J.-Y. Suh, Y.-S. Lee, B.-J. Lee. Atomistic Simulation of Hydrogen Diffusion at Tilt Grain Boundaries in Vanadium. Met. Mater. Int., 19:1221-1225, 2013.

[99] R. Matsumoto, M. Riku, S. Taketomi, N. Miyazaki. Hydrogen-Grain Boundary Interaction in Fe, Fe-C, and Fe-N Systems. Prog. Nuc. Science Tech., 2:9-15, 2011.

[100] M. Koyama, E. Akiyama, K. Tsuzaki, D. Raabe. Hydrogen-assisted failure in a twinninginduced plasticity steel studied under in situ hydrogen charging by electron channeling contrast imaging. Acta Mater., 61:4607-4618, 2013.

[101] W.-S. Ko, J.B. Jeon, C.-H. Lee, J.-K. Lee, B.-J. Lee. Intergranular embrittlement of iron by phosphorus segregation: an atomistic simulation. Modelling Simul. Mater. Sci. Eng., 21: 025012, 2013.

[102] R. Kirchheim, T. Mütschele, W. Kieninger, H. Gleiter, R. Birringer, T.D. Koblé. Hydrogen in Amorphous and Nanocrystalline Metals. Mater. Sci. Eng., 99:457-462, 1988.

[103] H. Schiechl, A. Winkler. Growth of a vanadium oxide layer on V(100) and its reactivity towards atomic hydrogen. Fresen, J. Anal. Chem., 371:342-347, 2001.

[104] J.F. Smith, D.T. Peterson. The H-V (Hydrogen-Vanadium) System. Bull. Alloy Phase Dia., 3:55-60, 1982.

[105] M.H. Maxelon. Segregation von Wasserstoff und Deuterium an Versetzungen in Palladium. Dissertation, Georg-August-Universität Göttingen, 2000.

[106] G. Petzow. Metallographisches, keramographisches, plastographisches Ätzen. Borntraeger, 2006.

[107] S. Thiébaut, B. Décamps, J.M. Pénisson, B. Limacher, A. Percheron Guégan. Tem study of the aging of palladium-based alloys during tritium storage. Journal of Nuclear Materials, 277:217-225, 2000.

[108] A. Barnoush, M. Asgari, R. Johnsen. Resolving the hydrogen effect on dislocation nucleation and mobility by electrochemical nanoindentation. Scripta Mater., 66:414-417, 2012.

[109] M.J. Cordill, N.R. Moody, W.W. Gerberich. The role of dislocation walls for nanoindentation to shallow depths. Int. J. Plasticity, 25:281-301, 2009.

[110] E. Epler. Mechanische Eigenschaften von Lithiumionen Batterieelektrodenmaterialien bei verschiedenen Ladezuständen. Dissertation, Georg-August-Universität Göttingen, 2015.

[111] A.P. Zhilyaev, T.G. Langdon. Using high-pressure torsion for metal processing: Fundamentals and applications. Prog. Mater. Sci., 53:893-979, 2008. 
[112] Y. Estrin, A. Vinogradov. Extreme grain refinement by severe plastic deformation: A wealth of challenging science. Acta Mater., 61:782-817, 2013.

[113] G. Sha, K. Tugcu, X.Z. Liao, P.W. Trimby, M.Y. Murashkin, R.Z. Valiev, S.P. Ringer. Strength, grain refinement and solute nanostructures of an Al-Mg-Si alloy (AA6060) processed by high-pressure torsion. Acta Mater., 63:169-179, 2014.

[114] J. Čížek, M. Janeček, O. Srba, R. Kužel, Z. Barnovská, I. Procházka, S. Dobatkin. Evolution of defects in copper deformed by high-pressure torsion. Acta Mater., 59:2322-2329, 2011.

[115] Y.H. Zhao, Y.T. Zhu, X.Z. Liao, Z. Horita, T.G. Langdon. Influence of stacking fault energy on the minimum grain size achieved in severe plastic deformation. Mat. Sci. Eng. A, 463: 22-26, 2007.

[116] Yu. Ivanisenko, R.K. Wunderlich, R.Z. Valiev, H.-J. Fecht. Annealing behaviour of nanostructured carbon steel produced by severe plastic deformation. Scripta Mater., 49:947-952, 2003.

[117] Q. Wei, Z.L. Pan, X.L. Wu, B.E. Schuster, L.J. Kecskes, R.Z. Valiev. Microstructure and mechanical properties at different length scales and strain rates of nanocrystalline tantalum produced by high-pressure torsion. Acta Mater., 59:2423-2436, 2011.

[118] G.B. Rathmayr, R. Pippan. Influence of impurities and deformation temperature on the saturation microstructure and ductility of HPT-deformed nickel. Acta Mater., 59:7228-7240, 2011.

[119] C. Borchers, C. Garve, M. Tiegel, M. Deutges, A. Herz, K. Edalati, R. Pippan, Z. Horita, R. Kirchheim. Nanocrystalline steel obtained by ball milling of iron and graphite subsequently compacted by high-pressure torsion. Acta Mater., 97:207-215, 2015.

[120] F. Dalla Torre, P. Spätig, R. Schäublin, M. Victoria. Deformation behaviour and microstructure of nanocrystalline electrodeposited and high pressure torsioned nickel. Acta Mater., 53:2337-2349, 2005.

[121] V.V. Stolyarov, Y.T. Zhu, T.C. Lowe, R.K. Islamgaliev, R.Z. Valiev. A two step SPD processing of ultrafine-grained titanium. Nanostruct. Mater., 11:947-954, 1999.

[122] T.W. Hansen, J.B. Wagner, R.E. Dunin-Borkowski. Aberration corrected and monochromated environmental transmission electron microscopy: challenges and prospects for materials science. Mater. Sci. Tech. Ser., 26:1338-1344, 2010.

[123] $\operatorname{Titan}^{\text {TM }}$ ETEM. FEI Company, 2013.

[124] D.B. Williams, C.B. Carter. Transmission Electron Microscopy. Springer Science+Business Media, 2009.

[125] I.M. Robertson, H.K. Birnbaum. An HVEM study of hydrogen effects on the deformation and fracture of nickel. Acta Metall. Mater., 34:353-366, 1986.

[126] M.S. Daw, M.I. Baskes. Semiempirical, Quantum Mechanical Calculation of Hydrogen Embrittlement in Metals. Phys. Rev. Lett., 25:1285-1288, 1983.

[127] M.S. Daw, M.I. Baskes. Embedded-atom method: Derivation and application to impurities, surfaces, and other defects in metals. Phys. Rev. B, 29:6443-6453, 1984.

[128] S.M. Foiles, M.S. Daw, M.I. Baskes. Embedded-atom-method functions for the fcc metals Cu, Ag, Au, Ni, Pd, Pt, and their alloys. Phys. Rev. B, 33:7983-7991, 1986. 
[129] J.E. Angelo, N.R. Moody, M.I. Baskes. Trapping of hydrogen to lattice defects in nickel. Modelling Simul. Mater. Sci. Eng., 3:289-307, 1995.

[130] M.I. Baskes, X. Sha, J.E. Angelo, N.R. Moody. Trapping of hydrogen to lattice defects in nickel. Modelling Simul. Mater. Sci. Eng., 5:651-652, 1997.

[131] X. Xu, M. Wen, S. Fukuyama, K. Yokogawa. Simulation of hydrogen embrittlement at crack tip in nickel single crystal by embedded atom method. Mater. Trans., 42:2283-2289, 2001.

[132] M. Wen, S. Fukuyama, K. Yokogawa. Atomistic simulations of hydrogen effect on dissociation of screw dislocations in nickel. Scripta Mater., 52:959-962, 2005.

[133] M. Wen, S. Fukuyama, K. Yokogawa. Cross-slip process in fcc nickel with hydrogen in a stacking fault: An atomistic study using the embedded-atom method. Phys. Rev. B, 75: 144110, 2007.

[134] M. Wen, L. Zhang, B. An, S. Fukuyama, K. Yokogawa. Hydrogen-enhanced dislocation activity and vacancy formation during nanoindentation of nickel. Phys. Rev. B, 80:094113, 2009.

[135] M. Wen, Z. Li. Thermally activated process of homogeneous dislocation nucleation and hydrogen effects: An atomistic study. Comp. Mater. Sci., 54:28-31, 2012.

[136] M. Wen, X.-J. Xu, S. Fukuyama, K. Yokogawa. Embedded-atom-method functions for the body-centered-cubic iron and hydrogen. J. Mater. Res., 16:3496-3502, 2001.

[137] W. Xie, X. Liu, W. Chen, H. Zhang. Hydrogen hardening effect in heavily deformed single crystal $\alpha$-Fe. Comp. Mater. Sci., 50:3397-3402, 2011.

[138] J. Song, W.A. Curtin. Mechanisms of hydrogen-enhanced localized plasticity: An atomistic study using a-Fe as a model system. Acta Mater., 68:61-69, 2014.

[139] X.W. Zhou, J.A. Zimmerman, B.M. Wong, J.J. Hoyt. An embedded-atom method interatomic potential for Pd-H alloys. J. Mater. Res., 23:704-718, 2008.

[140] H. Ogawa. Computational study of lattice defects in metal-hydrogen systems. J. Alloy Compd., 580:S131-S134, 2013.

[141] L. Verlet. Computer "Experiments" on Classical Fluids. I. Thermodynamical Properties of Lennard-Jones Molecules. Phys. Rev., 159:98-103, 1967.

[142] S. Nosé. A unified formulation of the constant temperature molecular dynamics methods. J. Chem. Phys., 81:511-519, 1984.

[143] W.G. Hoover. Canonical dynamics: Equilibrium phase-space distributions. Phys. Rev. A, 31:1695-1697, 1985.

[144] K. Durst, B. Backes, O. Franke, M. Göken. Indentation size effect in metallic materials: Modeling strength from pop-in to macroscopic hardness using geometrically necessary dislocations. Acta Mater., 54:2547-2555, 2006.

[145] W.C. Oliver, G.M. Pharr. An improved technique for determining hardness and elastic modulus using load and displacement sensing indentation experiments. J. Mater. Res., 7: 1564-1583, 1992.

[146] K.R. Morasch, D.F. Bahr. The effects of hydrogen on deformation and cross slip in a BCC titanium alloy. Scripta Mater., 45:839-845, 2001. 
[147] W. Wang, K. Lu. Nanoindentation study on elastic and plastic anisotropies of $\mathrm{Cu}$ single crystals. Phil. Mag., 86:5309-5320, 2006.

[148] H. Hertz. Ueber die Berührung fester elastischer Körper. J. Reine. Angew. Math., 92: $156-171,1881$.

[149] K.W. McElhaney, J.J. Vlassak, W.D. Nix. Determination of indenter tip geometry and indentation contact area for depth-sensing indentation experiments. J. Mater. Res., 13: 1300-1306, 1998.

[150] C.L. Kelchner, S.J. Plimpton, J.C. Hamilton. Dislocation nucleation and defect structure during surface indentation. Phys. Rev. B, 58:11085-11088, 1998.

[151] T. Zhu, J. Li, K.J. Van Vliet, S. Ogata, S. Yip, S. Suresh. Predictive modeling of nanoindentation-induced homogeneous dislocation nucleation in copper. J. Mech. Phys. Solids, 52:691-724, 2004.

[152] G. Ziegenhain. Atomistische Simulation von Nanoindentation. Dissertation, Technischen Universität Kaiserslautern, 2009.

[153] H. Kimizuka, H. Mori, S. Ogata. Effect of temperature on fast hydrogen diffusion in iron: A path-integral quantum dynamics approach. Phys. Rev. B, 83:094110, 2011.

[154] A. Stukowski. Visualization and analysis of atomistic simulation data with OVITO - the Open Visualization Tool. Modelling Simul. Mater. Sci. Eng., 18:015012, 2010.

[155] A. Ramasubramaniam, M. Itakura, E.A. Carter. Interatomic potentials for hydrogen in $\alpha$-iron based on density functional theory. Phys. Rev. B, 79:174101, 2009.

[156] D. Setman, M. Krystian, M.J. Zehetbauer. Lattice Defects in Hydrogenated and HPT Processed Pd. Mater. Sci. Forum, 584-586:355-360, 2008.

[157] M. Bönisch, M.J. Zehetbauer, M. Krystian, D. Setman, G. Krexner. Stabilization of Lattice Defects in HPT-deformed Palladium Hydride. Mater. Sci. Forum, 667-669:427-432, 2011.

[158] J.P. Hirth. Effects of Hydrogen on the Properties of Iron and Steel. Met. Mater. Trans. A, 11:861-890, 1980.

[159] D. Kuhlmann-Wilsdorf. The theory of dislocation-based crystal plasticity. Phil. Mag., 79: 955-1008, 1999.

[160] A. Barnoush, N. Kheradmand, T. Hajilou. Correlation between the hydrogen chemical potential and pop-in load during in situ electrochemical nanoindentation. Scripta Mater., 108:76-79, 2015.

[161] R. Matsumoto, S. Taketomi, S. Matsumoto, N. Miyazaki. Atomistic simulations of hydrogen embrittlement. Int. J. Hydrogen Energ., 34:9576-9584, 2009.

[162] S. Taketomi, R. Matsumoto, N. Miyazaki. Atomistic study of the competitive relationship between edge dislocation motion and hydrogen diffusion in alpha iron. J. Mater. Res., 26: 1269-1278, 2011.

[163] V. Gaspard, G. Kermouche, D. Delafosse, A. Barnoush. Hydrogen effect on dislocation nucleation in a ferritic alloy $\mathrm{Fe}-15 \mathrm{Cr}$ as observed per nanoindentation. Mat. Sci. Eng. A, 604:86-91, 2014.

[164] K.L. Johnson. Continuums mechanics. Cambridge University Press, 2003.

[165] MatWeb Material Property Data. http://www.matweb.com. Zugriff: 04.10.2015. 
[166] A. Christian, O. Kanert, J.Th.M. De Hosson. Dislocation dynamics in vanadium: a nuclear magnetic resonance and transmission electron microscopic study. Acta Metall. Mater., 38: 2479-2484, 1990.

[167] J. von Pezold, L. Lymperakis, J. Neugebeauer. Hydrogen-enhanced local plasticity at dilute bulk $\mathrm{H}$ concentrations: The role of $\mathrm{H}-\mathrm{H}$ interactions and the formation of local hydrides. Acta Mater., 59:2969-2980, 2011.

[168] M.D. Uchic, D.M. Dimiduk, J.N. Florando, W.D. Nix. Sample Dimensions Influence Strength and Crystal Plasticity. Science, 305:986-989, 2004.

[169] Z.W. Shan, R.K. Mishra, S.A. Syed Asif, O.L. Warren, A.M. Minor. Mechanical annealing and source-limited deformation in submicrometre-diameter ni crystals. Nat. Mater., 7:115119, 2008 .

[170] M.D. Uchic, P.A. Shade, D.M. Dimiduk. Plasticity of Micrometer-Scale Single Crystals in Compression. Annu. Rev. Mater. Res., 39:361-386, 2009.

[171] J.R. Greer, C.R. Weinberger, W. Cai. Comparing the strength of f.c.c. and b.c.c. submicrometer pillars: Compression experiments and dislocation dynamics simulations. Mat. Sci. Eng. A, 493:21-25, 2008.

[172] H.P. Barth. Einfluss von gelöstem Wasserstoff auf die Bildung und Multiplikation von Versetzungen bei der Verformung von Palladium. Diplomarbeit, Georg-August-Universität Göttingen, 2012.

[173] G.K Williamson, W.H Hall. X-ray line broadening from filed aluminium and wolfram. Acta Metall. Mater., 1:22 - 31, 1953.

[174] E.S. Fisher, D.G. Westlare, S.T. Ocker. Effects of Hydrogen and Oxygen on the Elastic Moduli of Vanadium, Niobium, and Tantalum Single Crystals. Phys. Status. Solidi. A, 28: 591-602, 1975.

[175] P. Sofronis, H.K. Birnbaum. Mechanics of the hydrogen-dislocation-impurity interactions I. increasing shear modulus. J. Mech. Phy. Solids, 43:49-90, 1995.

[176] A. Barnoush, H. Vehoff. In situ electrochemical nanoindentation: A technique for local examination of hydrogen embrittlement. Corros. Sci., 50:259-267, 2008.

[177] R. Kieselbach J. Woodtli. Correlation between the hydrogen chemical potential and pop-in load during in situ electrochemical nanoindentation. Eng. Fail. Anal., 7:427-450, 2000.

[178] A. Lehmberg. Untersuchung der Volumenänderung von Eisen nach Wasserstoffbeladung. Bachelorarbeit, Georg-August-Universität Göttingen, 2014.

[179] M. Roscher. Einfluss der Wasserstoffbeladung auf die mechanischen Eigenschaften von Metallen. Bachelorarbeit, Georg-August-Universität Göttingen, 2015.

[180] N. Brown, R.A. Ekvall. Temperature dependence of the yield points in iron. Acta Metall. Mater., 10:1101-1107, 1962.

[181] M.M. Hutchison. The temperature dependence of the yield stress of polycrystalline iron. Aust. Def. Scien. Serv., 121-127, 1962.

[182] R. Kirchheim, B. Somerday, P. Sofronis. Chemomechanical effects on the separation of interfaces occurring during fracture with emphasis on the hydrogen-iron and hydrogen-nickel system. Acta Mater., 87-98, 2015. 
[183] R. Kirchheim, U. Stolz. Modelling tracer diffusion and mobility of interstitials in disordered materials. J. Non-cryst. Solids, 70:323-341, 1985.

[184] R. Kirchheim. Interaction of hydrogen with dislocations in palladium - II. Interpretation of activity results by a Fermi-Dirac distribution. Acta Metall. Mater., 29:845-853, 1981.

[185] R. Kirchheim. Solid solutions of hydrogen in complex materials. In: H. Ehrenreich, F. Spaepen (Eds.), Solid State Physics, volume 59, 203-305. 2004.

[186] M. Lenz. Zusammenhang zwischen der Mikrostruktur und den mechanischen Eigenschaften sprengverschweißter Stähle. Bachelorarbeit, Georg-August-Universität Göttingen, 2013.

[187] M. Hammerschmidt. Mikrostruktur und Eigenschaften von Explosivschweißungen. Dissertation, Georg-August-Universität Göttingen, 1983. Fortschr.-Ber. Reihe 2 Nr. 52, VDI-Verlag Düsseldorf.

[188] K. Keller. Investigations of explosive cladding i. measurement of velocity of collision region. Z. Metallkd., 59:298-305, 1968.

[189] K. Keller. Investigation of explosive cladding ii. analysis of flow conditions. Z. Metallkd., 59:383-389, 1968.

[190] K. Keller. Investigations of explosive cladding iii. metallographic investigation and mechanical properties of explosive clads. Z. Metallkd., 59:503-514, 1968.

[191] C. Borchers, M. Lenz, M. Deutges, H. Klein, F. Gärtner, M. Hammerschmidt, H. Kreye. Microstructure and mechanical properties of medium-carbon steel bonded on low-carbon steel by explosive welding. Mater. Design, 89:369-376, 2016.

[192] S.-L. Chou, W.-T. Tsai. Hydrogen embrittlement of duplex stainless steel in concentrated sodium chloride solution. Mater. Chem. Phys., 60:137-142, 1999.

[193] M.L. Martin, J.A. Fenske, G.S. Liu, P. Sofronis, I.M. Robertson. On the formation and nature of quasi-cleavage fracture surfaces in hydrogen embrittled steels. Acta Mater., 59: 1601-1606, 2011.

[194] T. Doshida, M. Nakamura, H. Saito, T. Sawada, K. Takai. Hydrogen-enhanced lattice defect formation and hydrogen embrittlement of cyclically prestressed tempered martensitic steel. Acta Mater., 61:7755-7766, 2013.

[195] Y. Murakami, T. Kanezaki, P. Sofronis. Hydrogen embrittlement of high strength steels: Determination of the threshold stress intensity for small cracks nucleating at nonmetallic inclusions. Eng. Fract. Mech., 97:227-243, 2013.

[196] T. Depover, D. Pérez Escobar, E. Wallaert, Z. Zermout, K. Verbeken. Effect of hydrogen charging on the mechanical properties of advanced high strength steels. Int. J. Hydrogen Energ., 39:4647-4656, 2014.

[197] M. Koyama, H. Springer, S.V. Merzlikin, K. Tsuzaki, E. Akiyama, D. Raabe. Hydrogen embrittlement associated with strain localization in a precipitation-hardened Fe-Mn-Al-C light weight austenitic steel. Int. J. Hydrogen Energ., 39:4634-4646, 2014.

[198] S. Wang, M.L. Martin, P. Sofronis, S. Ohnuki, N. Hashimoto, I.M. Robertson. Hydrogeninduced intergranular failure of iron. Acta Mater., 69:275-282, 2014.

[199] H. Vehoff, H.-K. Klameth. Hydrogen embrittlement and trapping at crack tips in Ni-single crystals. Acta Metall. Mater., 33:955-962, 1985. 
[200] F. Lecoester, J. Chêne, D. Noel. Hydrogen embrittlement of the Ni-base Alloy 600 correlated with hydrogen transport by dislocations. Mat. Sci. Eng. A, 262:173-183, 1999.

[201] S. Bechtle, M. Kumar, B.P. Somerday, M.E. Launey, R.O. Ritchie. Grain-boundary engineering markedly reduces susceptibility to intergranular hydrogen embrittlement in metallic materials. Acta Mater., 57:4148-4157, 2009.

[202] D. Hardie, P. McIntyre. The low-temperature embrittlement of niobium and vanadium by both dissolved and precipitated hydrogen. Metall. Trans., 4:1247-1254, 1973.

[203] T.B. Massalski. Binary Alloy Phase Diagrams. ASM International, 1990. 


\section{Danksagung}

An dieser Stelle möchte ich mich bei all denen bedanken, die mir das Schreiben dieser Arbeit ermöglicht haben.

Mein besonderer Dank gilt Prof. Dr. Reiner Kirchheim für die Betreuung und Unterstützung während der gesamten Doktorarbeit. Er hat mir viele Reisen zu Konferenzen ermöglicht, die sehr interessant waren und mich in meiner Arbeit vorangebracht haben.

Bei Prof. Astrid Pundt möchte ich mich für ihre Betreuung und viele anregende Diskussionen bedanken.

Für die Übernahme des Koreferats bedanke ich mich bei Prof. Dr. Michael Seibt.

Herzlich möchte ich mich bei Dr. Christine Borchers für die vielen fruchtbaren Diskussionen und das unermüdliche Korrekturlesen vieler Arbeiten und Paper bedanken.

Bei Marie Tiegel möchte ich mich sowohl für die täglichen Diskussionen mit Kaffee, aus denen viele gute Ideen entstanden sind, bedanken, als auch für ihre Hilfe bei vielen Experimenten.

Mit meinen Bürokollegen Andreas Kelling und Johannes Maier hatte ich immer eine sehr gute Atmosphäre im Büro.

Für die ständige Hilfsbereitschaft bei Experimenten am FIB und TEM möchte ich mich auch bei Dr. May Martin bedanken.

Für die Hilfe bei den Untersuchungen am ETEM möchte ich mich bei Dr. Andreas Kelling bedanken. Bei Dr. Inga Knorr möchte ich mich für die Einweisung am Nanoindenter und Hilfe bei den Experimenten an Vanadiumsäulen bedanken. Für die Nutzung des in-situ Nanoindentationsaufbaus zur Untersuchung von Palladium-Wasserstofflegierungen bedanke ich mich bei Dr. Eike Epler. Dankbar bin ich auch Hans Peter Barth und Dr. Yuzeng Chen für die gemeinsamen Untersuchungen an kaltgewalztem Palladiumblech. Für die Beschichtung diverser Proben mit Palladium an der Sputteranlage der Arbeitsgruppe von Prof. Dr. Pundt möchte ich mich bei Magnus Hamm bedanken.

Viel zu verdanken habe ich auch den technischen Mitarbeitern des Instituts für Materialphysik der Universität Göttingen, die mir bei experimentellen Arbeiten zur Seite gestanden haben. Hier gilt mein Dank besonders den Mitarbeitern Annegret Lehmberg, Karin Ahlborn, Kerstin Born, Matthias Hahn und Dieter Plischke für die Lösung vieler experimenteller Probleme.

Auch bei Florian Oelkers und Frank Köhler möchte ich mich bedanken, für die stets sehr guten Versuchsaufbauten und die Diskussion neuer Konstruktionen.

Außerdem möchte ich mich herzlich bei Annegret Lehmberg, Malte Lenz und Moritz Roscher für die erfolgreiche Zusammenarbeit im Rahmen ihrer durch mich betreuten Bachelorarbeiten bedanken.

An dieser Stelle möchte ich mich bei meiner Verlobten Melinda Warncke für viele Aufmunterungen und das Korrekturlesen der Arbeit bedanken. Zu guter Letzt gilt mein besonderer Dank meiner Familie. Meinen Eltern, sowie meinen Geschwistern möchte ich an dieser Stelle dafür danken, dass sie mich auf diesem Weg begleitet haben und ich immer auf ihre Unterstützung bauen konnte. 


\section{Akademischer Lebenslauf}

\section{Persönliche Daten}

Name : Martin Dietmar Deutges

Geburtsort : Duisburg

Geburtsdatum : 11.08 .1985

Familienstand : ledig

Nationalität : deutsch

\section{$\underline{\text { Schulische Ausbildung }}$}

23.06.2005

Erwerb des Abiturs am Gymnasium Julianum in Helmstedt

\section{Zivildienst}

01.07.2005 - 30.06.2006 Ambulante Altenpflege bei der AWO Sozialstation Schöningen

\section{Wissenschaftliche Ausbildung}

01.10.2006 - 31.03.2012 Studium der Physik an der Georg-August-Universität Göttingen

20.04.2009 - 20.10.2009 Bachelorarbeit in der Arbeitsgruppe Prof. Dr. Reiner Kirchheim am Institut für Materialphysik

Thema „Transmissionselektronenmikroskopische Untersuchung von kaltverformtem und anschließend wärmebehandeltem perlitischem Stahl"

19.08.2010-24.04.2012 Masterarbeit in der Arbeitsgruppe Prof. Dr. Reiner Kirchheim am Institut für Materialphysik

Thema „Untersuchung des Einflusses von gelöstem Wasserstoff auf die plastische Verformung von Palladium und Vanadium mit Hilfe von Nanoindentation und Transmissionselektronenmikroskopie“"

01.06.2012 - 29.02.2016 Wissenschaftlicher Mitarbeiter in der Arbeitsgruppe Prof. Dr. Reiner Kirchheim am Institut für Materialphysik 


\section{Akademische Lehrtätigkeiten}

2007

$2008-2011$

2010

$2011 \& 2013$

2012

2013

2013

$2013-2014$

2014

$2014 \& 2015$

2015
Betreuung einer Übungsgruppe zur Vorlesung Einführung in die Programmierung in den Naturwissenschaften.

Anleitung von Studenten im Physikalischen Anfängerpraktikum.

Betreuung einer Übungsgruppe in Computer gestütztes wissenschaftliches Rechnen II.

Anleitung von Studenten in Fortgeschrittenenpraktikum Bachelor M3: Elektronenmikroskopie - vom Realraum zum reziproken Raum.

Betreuung einer Übungsgruppe zur Vorlesung Einführung in die Festkörper und Materialphysik.

Betreuung der Bachelorarbeit von Malte Lenz.

Anleitung von Studenten im Master-Forschungspraktikum PHA: Phasenumwandlungen in Eisen-Kohlenstoff-Legierungen.

Betreuung der Bachelorarbeit von Annegret Lehmberg.

Anleitung von Studenten im Fortgeschrittenenpraktikum Bachelor M5: Wasserstoff in Palladium.

Anleitung von Studenten im Master-Forschungspraktikum ATE: Analytische Transmissionselektronenmikroskopie von selbstorganisierten Nanokompositen.

Betreuung der Bachelorarbeit von Moritz Roscher. 